\title{
A General Perception Based Framework for Modelling Animal Aggregation
}

by

Jennifer Adele Schellinck, B.A., B.Sc.

A thesis submitted to

the Faculty of Graduate Studies and Research

in partial fulfilment of

the requirements for the degree of

\section{Doctor of Philosophy}

\author{
Institute of Cognitive Science \\ Carleton University \\ Ottawa, Ontario, Canada \\ September 2008
}

(C)2008 Jennifer Adele Schellinck 


$\begin{array}{ll}\begin{array}{l}\text { Library and } \\ \text { Archives Canada }\end{array} & \begin{array}{l}\text { Bibliothèque et } \\ \text { Archives Canada }\end{array} \\ \begin{array}{l}\text { Published Heritage } \\ \text { Branch }\end{array} & \begin{array}{l}\text { Direction du } \\ \text { Patrimoine de l'édition }\end{array} \\ \begin{array}{l}\text { 395 Wellington Street } \\ \text { Ottawa ON K1A 0N4 } \\ \text { Canada }\end{array} & \begin{array}{l}\text { 395, rue Wellington } \\ \text { Ottawa ON K1A 0N4 } \\ \text { Canada }\end{array}\end{array}$

Your file Votre référence ISBN: 978-0-494-47490-7

Our file Notre référence

ISBN: 978-0-494-47490-7

NOTICE:

The author has granted a nonexclusive license allowing Library and Archives Canada to reproduce, publish, archive, preserve, conserve, communicate to the public by telecommunication or on the Internet, loan, distribute and sell theses worldwide, for commercial or noncommercial purposes, in microform, paper, electronic and/or any other formats.

The author retains copyright ownership and moral rights in this thesis. Neither the thesis nor substantial extracts from it may be printed or otherwise reproduced without the author's permission.
AVIS:

L'auteur a accordé une licence non exclusive permettant à la Bibliothèque et Archives Canada de reproduire, publier, archiver, sauvegarder, conserver, transmettre au public par télécommunication ou par l'Internet, prêter, distribuer et vendre des thèses partout dans le monde, à des fins commerciales ou autres, sur support microforme, papier, électronique et/ou autres formats.

L'auteur conserve la propriété du droit d'auteur et des droits moraux qui protège cette thèse. $\mathrm{Ni}$ la thèse ni des extraits substantiels de celle-ci ne doivent être imprimés ou autrement reproduits sans son autorisation.
In compliance with the Canadian Privacy Act some supporting forms may have been removed from this thesis.

While these forms may be included in the document page count, their removal does not represent any loss of content from the thesis.
Conformément à la loi canadienne sur la protection de la vie privée, quelques formulaires secondaires ont été enlevés de cette thèse.

Bien que ces formulaires aient inclus dans la pagination, il n'y aura aucun contenu manquant.

\section{Canada}




\section{Abstract}

Animal aggregates (sparrow flocks, anchovy schools, caribou herds) are complex systems potentially made up of thousands of individuals moving in a coordinated mass. While such aggregates are difficult to study in the field, individual based spatially explicit computer models (IBSEMs) offer researchers a way to investigate how the interplay of individual behaviours makes aggregation possible and leads to different aggregate level behaviours. In this thesis I argue that in order to model species-specific aggregate behaviours, modellers must pay attention to three properties of animals that uniquely determine their aggregating behaviour: the perceptual mechanisms of the animal, the movement abilities of the animal and the cognitive mechanisms that connect perception to action. The existing modelling framework, the Attraction Repulsion (AR) framework, does not allow for an adequate representation of these three facets of individual aggregating behaviour. As an alternative I present a new modelling framework, the Perception Cognition Action (PCA) framework. I use this framework to create an experimentally based, experimentally validated computer model of Danio rerio aggregate behaviour. I further demonstrate, more generally, the importance of modelling perception by analysing the behaviour of a theoretical aggregate model that varies the perceptual abilities of agents and determines the effect that this has on aggregate cohesion. Lastly, I explore the interplay between perception, cognition and movement by varying individual perception, cognition and action properties. I analyse the behaviour of the resulting aggregate with a focus on how the interaction between perception, cognition and action determines aggregate properties. 


\section{Acknowledgments}

I would like to acknowledge Colin Henein for his encouragement, patience and understanding and Tony White for his interest, perseverance, financial support and unflagging willingness to meet and discuss cognitive science in coffee shops.

I would like to thank the Godin lab for their generous assistance and marvellous enthusiasmin particular Stacey Lee-Jenkins for introducing me to the world of fish research and Kim Hair for her fish care and words of wisdom. I would like to thank Dr. Danny Grünbaum for rounding up the Tracker 3D software and answering my installation questions, Dr. Mark Forbes for giving me a new and useful perspective on computer modelling, Dr. Tom Sherratt for getting me started on the experimental research front and Dr. Stephen Cooke for lending me his lab space and enabling my project to continue.

I would also like to thank Heather and Tony Schellinck for their big picture perspective, reassurance, financial assistance and practical advice and Kringen and Jean-Claude Henein for their emotional support. 


\section{Table of Contents}

$\begin{array}{ll}\text { Abstract } & \text { iii }\end{array}$

Acknowledgments $\quad$ iv

Table of Contents $\quad$ v

List of Tables $\quad$ x

List of Figures $\quad$ xii

List of Algorithms $\quad$ xiv

List of abbreviations and symbols $\quad$ xv

1 Introduction 1

1.1 Aggregate research: A synopsis . . . . . . . . . . . . . . 1

1.2 Thesis goals and contributions: The Perception Cognition Action (PCA) modelling

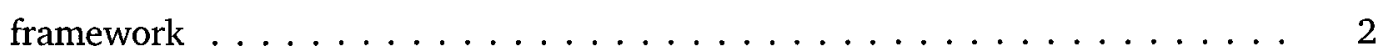

1.3 Thesis overview $\ldots \ldots \ldots \ldots \ldots \ldots \ldots \ldots \ldots \ldots \ldots \ldots \ldots$

2 Animal aggregate research: An overview with an emphasis on aggregate mod$\begin{array}{ll}\text { elling } & 6\end{array}$

2.1 Definition and properties of animal aggregates $\ldots \ldots \ldots \ldots \ldots$

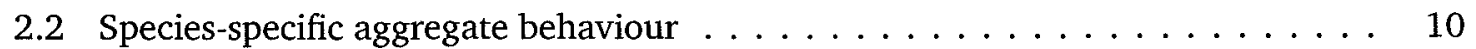

2.2.1 The individual underpinnings of aggregate behaviour . . . . . . . 10 
2.2.2 Aggregate-level findings, with a focus on research methods . . . . . . . 13

2.3 Modelling aggregation $\ldots \ldots \ldots \ldots \ldots \ldots \ldots \ldots \ldots \ldots$

2.3.1 Breder's model: Drawing an analogy with physical forces . . . . . . . 17

2.3.2 A typical aggregate model . . . . . . . . . . . . . . . . . . . . . 19

2.3.3 Recent developments in animal aggregate models . . . . . . . . . . 21

2.4 Current findings by aggregate models . . . . . . . . . . . . . . . 23

2.4.1 Findings of mathematical models . . . . . . . . . . . . . . 23

2.4.2 Findings of individually based, spatial explicit computer models (IBSEMs) 26

2.5 Species-specific models of aggregation . . . . . . . . . . . . . 34

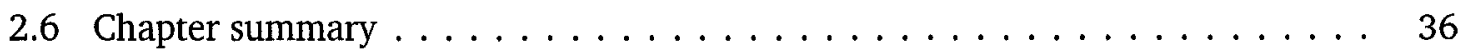

3 Issues with the Attraction Repulsion framework and a proposed solution- the $\begin{array}{ll}\text { Perception Cognition Action framework } & 38\end{array}$

3.1 Methodological issues with the AR framework . . . . . . . . . . . . 39

3.1.1 Model Construction using the AR framework . . . . . . . . . . . . . 39

3.1.2 Problems with Resulting Aggregate Models . . . . . . . . . . . . . . . . 41

3.2 Moving Forward: A cognitive science solution $\ldots \ldots \ldots \ldots$

3.2.1 The problem of verifying models $\ldots \ldots \ldots \ldots \ldots \ldots$

3.2.2 The implicit role of information processing in the AR framework . . . . 46

3.2.3 Updating the AR framework using the information processing paradigm 48

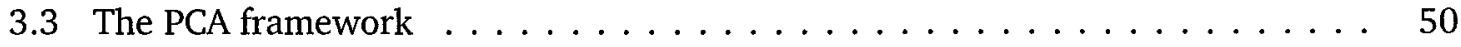

3.3.1 Framework Requirements $\ldots \ldots \ldots \ldots \ldots \ldots$

3.3.2 Details of the Modelling Framework . . . . . . . . . . . . . 51

3.4 Problems Solved By the PCA Framework . . . . . . . . . . . . . . . . . . . 59

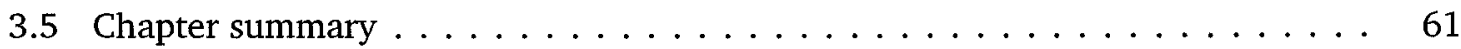

4 Testing the Perception Cognition Action framework: A D. rerio model 63

4.1 Implementing the PCA framework in Breve $\ldots \ldots \ldots \ldots \ldots$. . . . . . . 64

4.1.1 Physical object implementation . . . . . . . . . . . . . . 64

4.1 .2 Agents ............................... 65 
4.1.3 Agent behaviour during one timestep . . . . . . . . . . 66

4.1.4 Information available to agents: Agent perception and memory . . . . . 66

4.1 .5 Agent cognition . . . . . . . . . . . . . . . 68

4.1 .6 Agent movement . . . . . . . . . . . . . . . . . . . . . . . . . 69

4.2 Gathering data on $D$. rerio behaviour and properties $\ldots \ldots \ldots \ldots$

4.2 .1 Methodology . . . . . . . . . . . . . . . . . 70

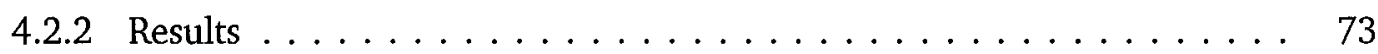

4.3 Creating an IBSEM model of $D$. rerio aggregation $\ldots \ldots \ldots \ldots \ldots \ldots$

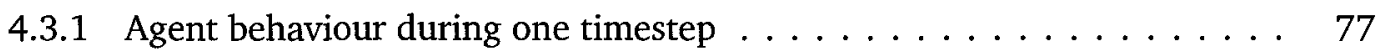

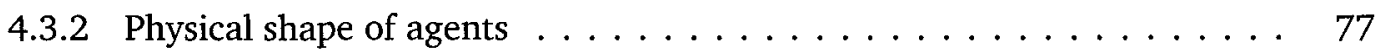

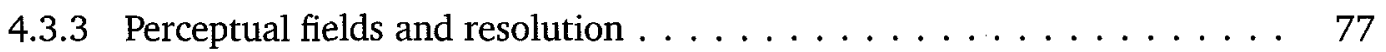

4.3.4 Cognitive algorithms for determining movement . . . . . . . . . . 80

4.3 .5 Movement . . . . . . . . . . . . . . . . . . 84

4.4 Model analysis, results and predictions . . . . . . . . . . . . 86

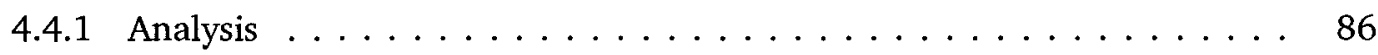

4.4 .2 Results and predictions . . . . . . . . . . . . . 88

4.5 Comparison of PCA model predictions with actual aggregate behaviour and behaviour of simple AR model . . . . . . . . . . . . . . . . . . . . . 92

4.5.1 Collection and analysis of aggregate data . . . . . . . . . 93

4.5.2 Comparison of aggregate behaviour with model predictions . . . . . . 95

4.5.3 Comparison of aggregate behaviour with behaviour of simple AR model . 97

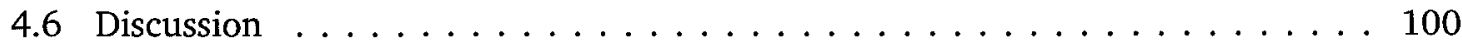

4.7 Chapter summary . . . . . . . . . . . . . . . . . 103

5 Perceptual underpinnings of aggregate behaviour 104

5.1 An information based definition of aggregation . . . . . . . . . . 105

5.2 Four scenarios exploring the minimum information required for aggregation . . 107

5.2.1 Model setup and methodology . . . . . . . . . . . . . . . 108

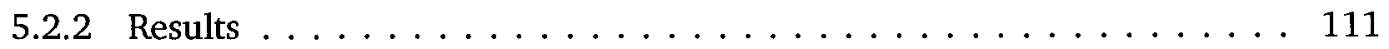


5.2 .3 Discussion . . . . . . . . . . . . . . . . . . . . . . 112

5.3 Five scenarios exploring the influence of perception on aggregation $\ldots \ldots \ldots$

5.3.1 Model setup and methodology . . . . . . . . . . . . . . . . 116

5.3 .2 Model results . . . . . . . . . . . . . . . . . . . . . . . . . . . 120

5.3 .3 Discussion . . . . . . . . . . . . . . . . . . . . . . . 122

5.4 Chapter summary . . . . . . . . . . . . . . . . . . . . . 126

6 The influence of individual cognition and movement on aggregate movement, $\begin{array}{ll}\text { alignment and volume } & 128\end{array}$

6.1 Types of cognition required for aggregation $\ldots \ldots \ldots \ldots \ldots \ldots \ldots \ldots$

6.2 The aggregate as a complex system: Predicting interactions between cognition, movement and perception . . . . . . . . . . . . . . 130

6.3 Modelling the interaction between cognition, movement and perception . . . . . 132

6.3 .1 Setup and methodology $\ldots \ldots \ldots \ldots \ldots \ldots \ldots$

6.3 .2 Model results . . . . . . . . . . . . . . . . . . . . . . . . 135

6.3 .3 Discussion . . . . . . . . . . . . . . . . . . 143

6.4 Chapter summary . . . . . . . . . . . . . . . . . . . . . . 147

7 Conclusions and future work $\quad 148$

7.1 Species-specific modelling with the PCA framework: A summary of thesis contri-

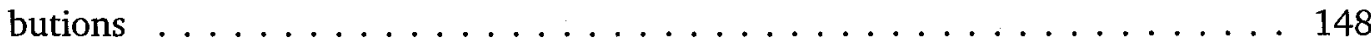

7.2 Limitations of current work and future research $\ldots \ldots \ldots \ldots \ldots$. . . . . . . 151

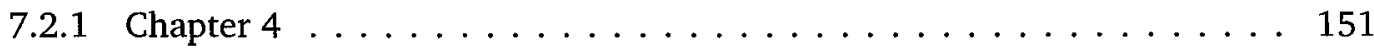

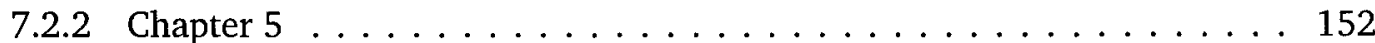

7.2 .3 Chapter $6 \ldots \ldots \ldots \ldots \ldots \ldots \ldots \ldots$. . . . . . . . . . . . . . . . . . . . . .

7.3 Moving towards an understanding of species-specific aggregation $\ldots \ldots \ldots \ldots 154$

$\begin{array}{ll}\text { References } & 156\end{array}$

Appendix A Maximum, Minimum and Mean Values for all 16 Parameter Combinations of The Danio rerio Model 163 


\section{List of Tables}

1 Speed data for D. rerio within fish and across all fish. . . . . . . . . . . 74

2 Turning angle data for D. rerio, within fish and across all fish. . . . . . . . 74

3 A summary of the individual distance data for fish moving in groups of four. . . 75

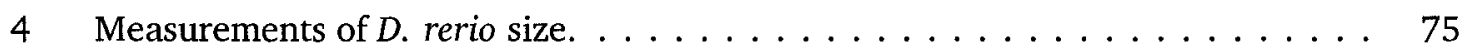

5 Parameters used to investigate model behaviour. . . . . . . . . . . . . . 84

6 A list of the 16 parameter combinations. . . . . . . . . . . . . 85

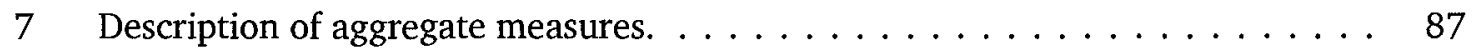

8 A summary of the model values across parameter combinations for aggregate measures of interest. . . . . . . . . . . . . . . . . . 89

9 Group measures of the behaviour of 18 D. rerio. . . . . . . . . . . . . 94

10 Parameter values for the four information scenarios. . . . . . . . . . . 110

11 Clusters relative to number of neighbours perceived by agents. . . . . . . . . 111

12 A comparison of behaviour between model runs. . . . . . . . . . . . . . 113

13 Model parameters for the perceptual field scenarios. . . . . . . . . . . . . . . 119

14 Cohesion of aggregates with different perceptual field shapes. . . . . . . . . 121

15 Predicted relationship between individual properties and aggregate properties. . 132

16 List of parameter sets used for prey and predator scenarios. . . . . . . . . . . . 134

17 An overview of prey and predator behaviour. . . . . . . . . . 136

18 Aggregate cohesion in the absence of centring, vector alignment and/or spacing. 137

19 Factors that interact for each measure. . . . . . . . . . . . . . . . . . . 139

20 Prey: Relationship between individual properties and aggregate properties. . . 140

21 Predator: Relationship between individual properties and aggregate properties. 140 
22 Actual and predicted relationships between individual properties and aggregate properties, for the prey and predator scenarios. . . . . . . . . . . . . 141

23 Comparison of prey and predator linear regression models, with respect to the relationship between individual properties and aggregate properties. . . . . . . 142

24 Correlation between aggregate measures in the prey scenario. . . . . . . . . . 142

25 Correlation between aggregate measures in the predator scenario. . . . . . . . . 143

26 Minimum values for Danio rerio model . . . . . . . . . . . . . . . . . . . . . 164

27 Average values for Danio rerio model . . . . . . . . . . . . . . . . . . 165

28 Maximum values for Danio rerio model . . . . . . . . . . . . . . . . . 166

29 Minimum values for simple Attraction Repulsion model f . . . . . . . . . . . 167

30 Average values for simple Attraction Repulsion model . . . . . . . . . . . . . 168

31 Maximum values for simple Attraction Repulsion model . . . . . . . . . . . . . 169

32 Prey: Linear model of relationship between individual properties and aggregate properties . . . . . . . . . . . . . . . . . . . . 170

33 Predator: Linear model of relationship between individual properties and aggregate properties $\ldots \ldots \ldots \ldots \ldots \ldots \ldots \ldots \ldots \ldots \ldots$ 


\section{List of Figures}

1 Using a simple density measure to define the concept of an animal aggregate. . . 7

2 Properties of an individual in an aggregate $\ldots \ldots \ldots \ldots$

3 Measuring group properties of an aggregate . . . . . . . . . . . 9

4 Organisms are both attracted to and repelled by other organisms. . . . . . . . 11

5 Calculating the behaviour of individuals within the aggregate over time. . . . 20

6 Individual rules, operating with particular zones of behaviour. . . . . . . . 27

7 Information processing: Information, represented by the dotted line, is received through the senses. . . . . . . . . . . . . . . . 45

8 Combining the concept of the information area with what is known about animal perception. . . . . . . . . . . . . . . . . . . . . . 49

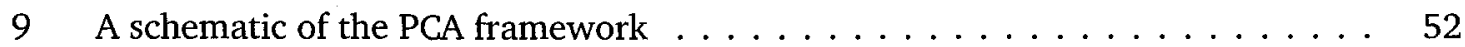

10 An agent's perceptual field $\ldots \ldots \ldots \ldots \ldots \ldots \ldots \ldots \ldots \ldots \ldots$

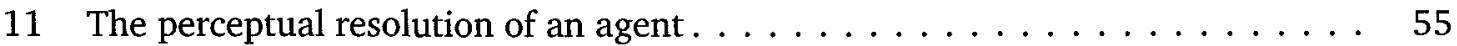

12 An overview of the cognitive structure. . . . . . . . . . . 57

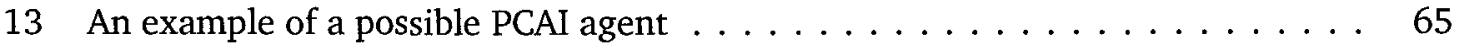

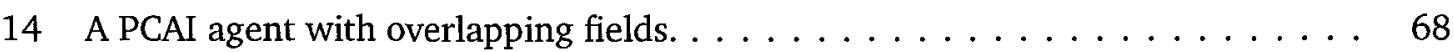

15 A PCAI agent with memory, attention and cognitive processing algorithms. . . . 69

16 Tracker 3D aquarium set up. Numbers in brackets are the interior dimensions of the tank, including coroplast $\ldots \ldots \ldots \ldots \ldots \ldots \ldots \ldots \ldots$

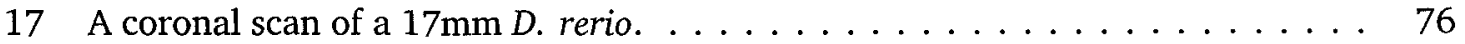

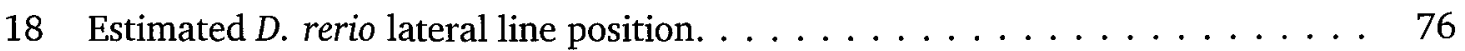

19 Agent shape, compared with $D$. rerio shape. . . . . . . . . . . . . . . . . 79 
20 Visual and lateral line fields of the agent. . . . . . . . . . . . . 81

21 An explanation of how aggregate measures are generated. . . . . . . . . . 88

22 Aggregate volume, speed and angle during a single run of the PCA D. rerio model . 91

23 Aggregate volume, speed and angle for a $D$. rerio aggregate of $18 D$. rerio. . . . 96

24 Aggregate volume, speed and angle during a single run of the simple $D$. rerio model for parameter combination $(10,1,3,3) \ldots \ldots \ldots$. . . . . . . . . 99

25 Animal aggregation, as represented by an aggregate information graph. . . . 106

26 Shape of the 5 perceptual fields (not to scale) $\ldots \ldots \ldots \ldots \ldots \ldots \ldots$

27 Narrow perceptual fields (shaded grey) can easily pass out of the densest part of

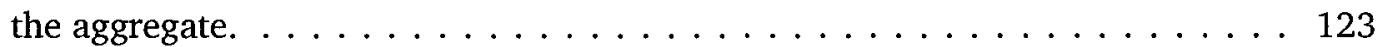

28 Two agents perceiving the same aggregate will receive different information. . . 124

29 The agent on the left, with the wide fields (shaded grey) perceives some agents. 124

30 Perceived nearest neighbours and actual nearest neighbours. . . . . . . . . 125 


\section{List of Algorithms}

1 PCAI agent behaviour during one timestep $\ldots \ldots \ldots \ldots \ldots \ldots \ldots$

$2 \quad$ D. rerio agent behaviour during one timestep. . . . . . . . . . . 78

3 Agent behaviour during one timestep of the minimum information models. . . . 109

4 Agent behaviour during one timestep for the perceptual field models. . . . . . . 118 


\title{
List of abbreviations and symbols
}

\author{
PCA \\ Perception Cognition Action framework \\ AR \\ Attraction Repulsion framework \\ PCAI \\ Perception Cognition Action implementation \\ IBSEM \\ Individually Based Spatially Explicit Model \\ $a$ \\ the attractive force between two individuals (in Breder's \\ equations) \\ $r$ \\ the repelling force between two individuals (in Breder's \\ equations) \\ $d$ \\ the distance between the individuals (in Breder's equa- \\ tions) \\ $c$ \\ a measure of the cohesiveness of the school as a whole \\ (in Breder's equations) \\ $r_{1}$ \\ the repulsion of fish 1 (or school 1) (in Breder's equa- \\ tions) \\ $r_{2}$ \\ the repulsion of fish 2 (or school 2) (in Breder's equa- \\ tions) \\ $i$ \\ an individual
}


$R$

$T$

$A\left(R_{1}, R_{2}, \cdots, R_{n}\right)$

$C(A, M)$ another individual

time

the position vector of a neighbour, $j$, at time $t$

the number of neighbours within the specified distance, $r$ at time $t$

the velocity of a neighbour, $j$, at time $t$

the neighbours within a particular distance o from the individual (but not closer than $r$ )

the neighbours that are within a particular distance, $a$ from the individual (but not closer than a distance $o$ )

a perceptual field

a point

the set of perceptual field vectors

the vertex of the perceptual field cone

a unit length direction vector

is the radius of the perceptual field cone

a perceptual resolution tuple

the set of perceptual resolution tuples

a transformation of the perceptual field vectors, $P$

attention function

cognitive processing function 


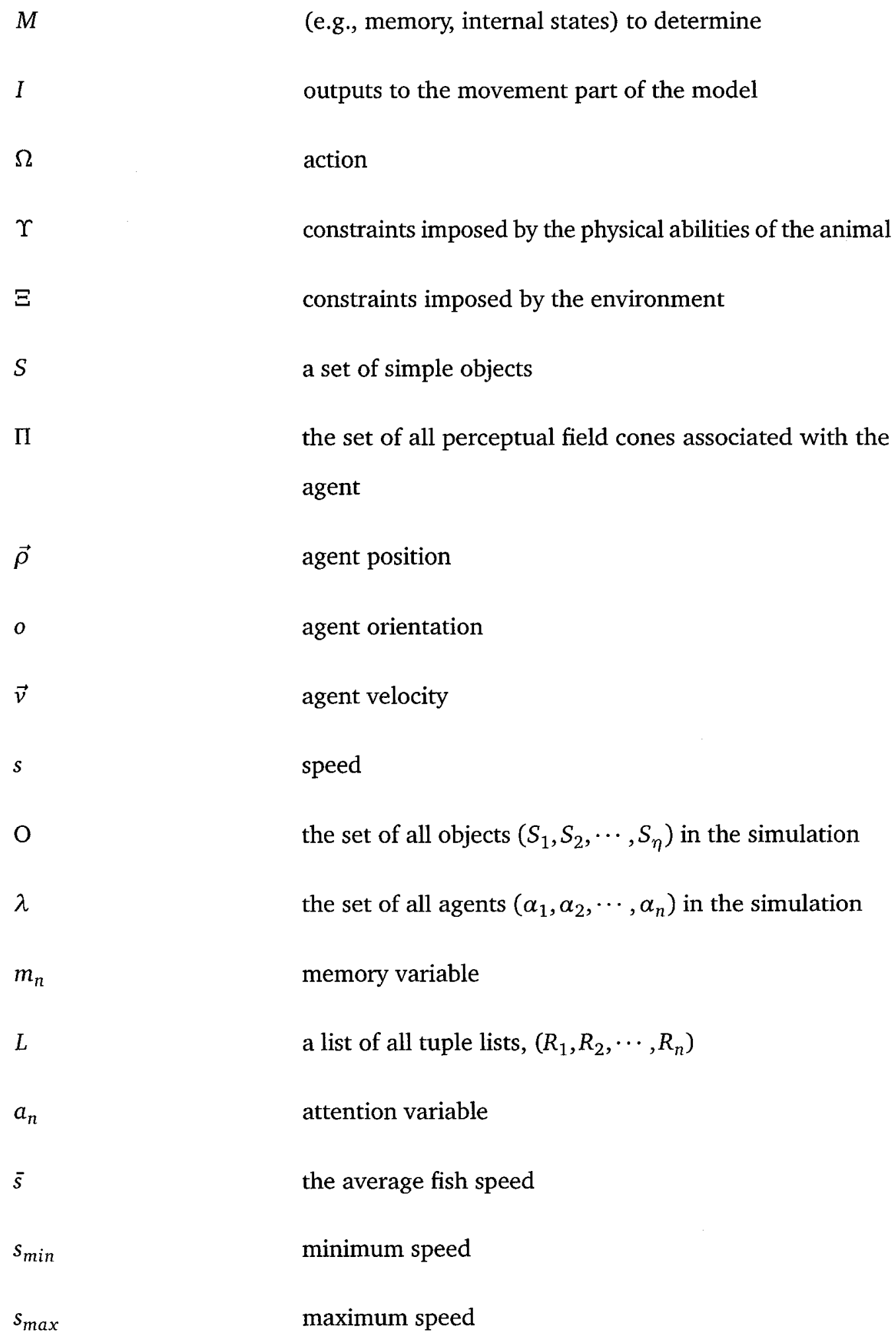




\begin{tabular}{|c|c|}
\hline$\overline{\text { ind }}$ & the average of the individuals values \\
\hline all & values for data pooled across all fish \\
\hline $\bar{\theta}$ & the mean turning angle \\
\hline$\theta_{\min }$ & the minimum turning angle \\
\hline$\theta_{\max }$ & the maximum turning angle \\
\hline close & $\begin{array}{l}\text { Average distance from a fish to its nearest neighbour in } \\
\text { the group over time }\end{array}$ \\
\hline fiar & $\begin{array}{l}\text { average distance from a fish to its farthest neighbour in } \\
\text { the group over time }\end{array}$ \\
\hline dist & $\begin{array}{l}\text { the across group average of the average distance of a fish } \\
\text { to its neighbours in the group over time }\end{array}$ \\
\hline grōup & the average of group values \\
\hline $\min _{\text {all }}$ & minimum across all fish \\
\hline $\max _{\text {all }}$ & the maximum across all fish \\
\hline $\overrightarrow{v_{c}}$ & the centring vector \\
\hline $\overrightarrow{v_{a}}$ & the velocity vector \\
\hline $\overrightarrow{v_{s}}$ & the spacing vector \\
\hline $\overrightarrow{v_{n}}$ & the new movement vector \\
\hline$\alpha_{i}$ & agent $i$ \\
\hline$\delta$ & $\begin{array}{l}\text { all agents who are within (closer or equal to) a preferred } \\
\text { spacing from the agent. }\end{array}$ \\
\hline
\end{tabular}
spacing from the agent. 


\begin{tabular}{|c|c|}
\hline$v_{r}$ & $\begin{array}{l}\text { a random vector influence, that represents natural vari- } \\
\text { ability in movement }\end{array}$ \\
\hline$\vec{\phi}$ & a final acceleration vector \\
\hline$w_{c}$ & the centring weight \\
\hline$w_{v}$ & velocity weight \\
\hline$w_{s}$ & spacing weight \\
\hline$w_{r}$ & random weight \\
\hline space $_{\text {pref }}$ & preferred spacing \\
\hline$s_{\max }$ & maximum speed \\
\hline$t \theta_{\max }$ & maximum turning angle \\
\hline$v_{\text {simp }}$ & simple volume \\
\hline $\exp$ & expanse \\
\hline$p$ & polarity \\
\hline$n_{\text {groups }}$ & number of groups \\
\hline$n_{\text {strag }}$ & number of stragglers \\
\hline$n_{\text {clust }}$ & number of clusters \\
\hline$s_{a g g}$ & aggregate speed \\
\hline$t \theta_{a g g}$ & aggregate turning angle \\
\hline range $\max$ & $\begin{array}{l}\text { The range of average maximum values across all } 16 \text { pa- } \\
\text { rameter combinations }\end{array}$ \\
\hline
\end{tabular}




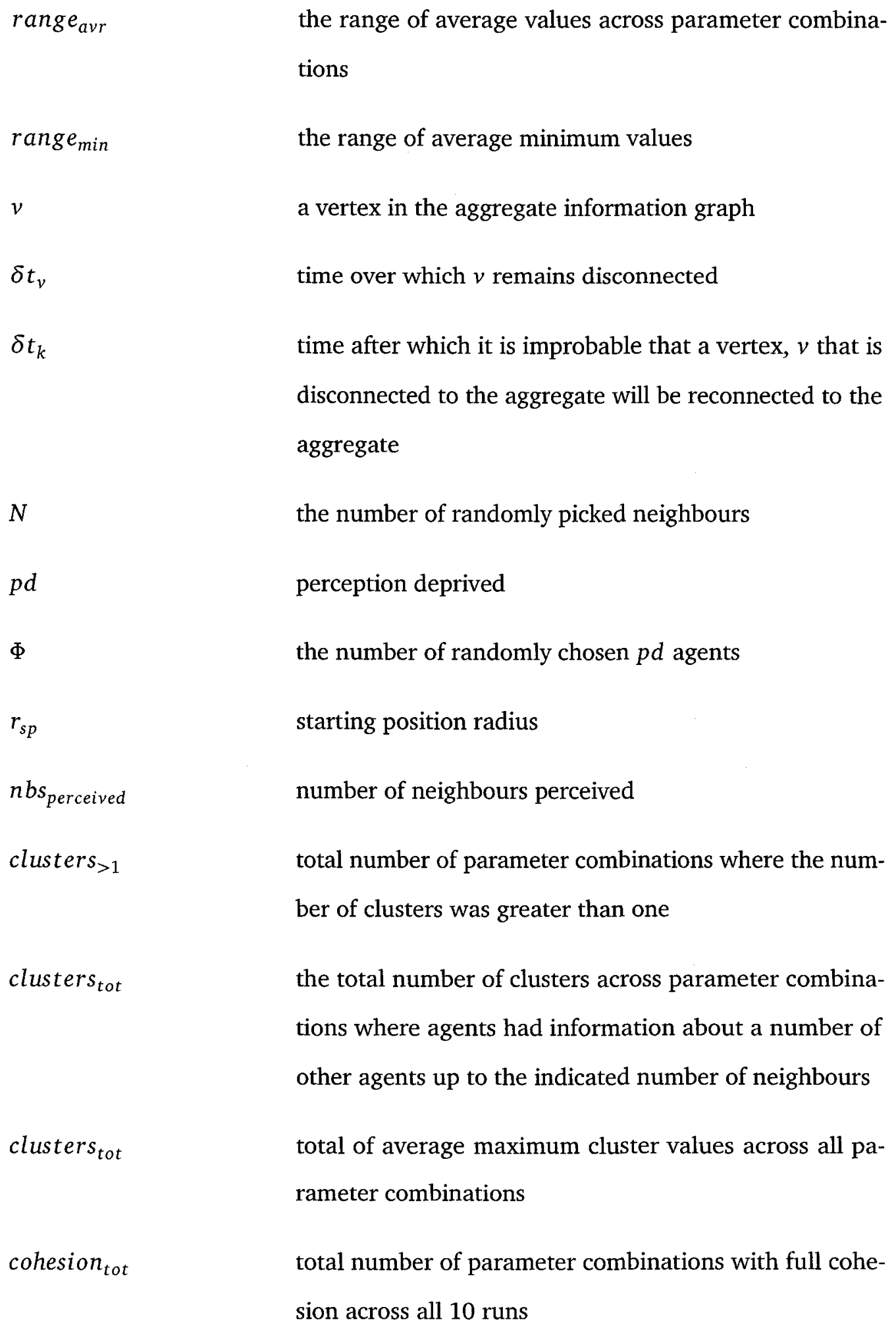


none $_{\text {perceived }}$

tot $_{\text {perceived }}$

$v_{p f}$ average number of agents that perceived no neighbours average value of the total perceived neighbours for each parameter combination

perceptual field volume in $\mathrm{cm}^{3}$ 


\section{Chapter 1}

\section{Introduction}

This chapter begins by providing a brief overview of aggregate research in order to situate the thesis in the broader context of the aggregate research field. The chapter then presents the contributions and goals of the thesis. It concludes with an overview of the remainder of the thesis.

\subsection{Aggregate research: A synopsis}

Animal aggregation is a pervasive phenomenon in the natural world. European Starlings move through the air in a tightly packed, fast moving group (Heppner, 1997). Slime mould live as a group of unconnected single-celled organisms until these organisms start to starve, at which point they gather together to form a single slug that moves as a cohesive whole (Camazine, Deneubourg, Franks, Sneyd, Bonabeau, and Theraulaz, 2003 ). Dolphins move in pods that perform elaborate manoeuvres in order to herd fish (Bel'kovich,1991), which themselves move in large, coordinated schools (Parrish, Viscido and Grünbaum, 2002).

Despite its prevalence, aggregation is not an inevitable result of organism interaction, but a behaviour that must be initiated and maintained through the action of specific biological mechanisms. Some aggregating animals, like dolphins, are highly complicated and intelligent organisms, while others, like slime mould cells, could barely be said to have cognitive abilities. Nonetheless, every one of these organisms has the ability to move together with other organisms of the same type in complicated ways to form cohesive, organised groups that often 
display elaborate spatial and temporal group patterns. This suggests that aggregation is both an evolutionarily important and multiply realisable natural behaviour.

Animal aggregate researchers (a interdisciplinary group comprised of cognitive scientists, psychologists, biologists, sociologists, computer scientists and physicists), have as their goal the description, explanation and prediction of the behaviour of animal aggregates. Researchers in this field may study the movement patterns generated by animal aggregates (for example, the swirling cylindrical patterns (mills) seen in fish schools (Parr, 1927)) or the patterns of communication that are generated by these aggregates over time (for example, the synchronised flashing of fireflies, (Moiseff and Copeland, 2000)). They may be interested in describing the nature of the aggregate behaviours seen in a particular species (for example, the 'people streams' observed in human crowd situations (Helbing, Farkas, Molnár and Vicsek, 2002)) or they may be interested in doing more abstract, theoretical work (for example, studying the effect that access to varying levels of environmental information has on general aggregate behaviour (Couzin, Krause, Franks and Levin, 2005)).

\subsection{Thesis goals and contributions: The Perception Cognition Ac- tion (PCA) modelling framework}

Thesis Goals The central premise of this thesis is that the existing theoretical framework for modelling animal aggregates- referred to in this thesis as the Attraction Repulsion (AR) framework- needs to be revised for two reasons. First, the AR framework lacks the theoretical power required to describe how individual behaviours and properties exbited by members of a particular species lead to the aggregate behaviour and properties of that species. Second, the AR framework is not adequate for creating predictive, verifiable models of specific animal aggregates.

Related to these issues of the AR framework, the goals of this thesis are as follows: 
- to present a new, perception based, information processing driven, theoretical framework for modelling animal aggregates — the Perception Cognition Action (PCA) frameworkfor use in creating species-specific aggregate models

- to explain how the PCA framework is compatible with, but usefully extends, the existing AR framework

- to demonstrate that the PCA framework can be fully implemented in an IBSEM modelling environment (the PCA Implementation (PCAI)), resulting in embodied, three-dimensional aggregates of computer agents

- to demonstrate the utility of the PCA framework, as implemented, by applying it both experimentally and theoretically.

Thesis Research Contributions Aggregation is a complex phenomenon, arising out of the combined actions of many individuals. Consequently, in order to answer questions about aggregation some understanding must be gained about the ways in which the different behaviours of individuals within an aggregate lead to aggregate-level behaviour. Models, particularly Lagrangian and individual based models (IBMs), are well positioned to investigate these questions because they can provide insight into the connections between the two levels of behaviour. With this in mind, this thesis presents an individual based, information processing driven, animal aggregate modelling framework for use in modelling species-specific aggregation. This frameworkthe PCA framework - will improve on the existing de facto aggregate modelling framework, referred to in this thesis as the Attraction Repulsion (AR) framework, by more concretely relating the relevant species-specific properties of individuals within an aggregate to the behaviour of the aggregate as a whole. In particular, the PCA framework's focus on the perceptual abilities of animals will allow biologists to more easily create experimentally verifiable individual based spatially explicit models (IBSEMs) of aggregation in specific species of animals.

After defining the PCA framework, the thesis will present the results of three research projects undertaken to demonstrate the utility of the PCA framework. In the first project, the PCA framework is implemented in the Breve modelling environment. Then, video tracking 
technology (Tracker 3D, Grünbaum, Viscido and Parrish, 2005) is used to collect data on the behaviour of individuals in a Danio rerio (common name zebra danio or zebrafish) aggregate and this data used in conjunction with the Breve implementation of the PCA framework to create a computer model of $D$. rerio aggregate behaviour, which is then compared with the behaviour of the actual $D$. rerio aggregate. As part of this project, the behavioural predictions of a conventional AR model are directly compared with the behavioural predictions from a model created using the proposed PCA framework.

With the predictive utility of the PCA framework established, the second project uses the PCA framework to examine the role that perception plays in determining properties of aggregate behaviour. The third project considers the interaction between perceptual, cognitive and movement properties, with an emphasis on the interaction between perceptual and cognitive properties, and how these individual properties combine to generate group-level aggregate properties.

Thus, the contributions the thesis will make to animal aggregate research are: a new modelling framework that will allow researchers to model the behaviour of specific animal aggregates (discussed in Chapter 3), an experimentally supported model of how individual $D$. rerio properties and behaviours lead to $D$. rerio aggregate behaviour (discussed in Chapter 4), a method for creating physically realistic, perception mediated interactions between three dimensional, embodied agents (discussed in Chapter 4), a preliminary explanation of the role of animal perception in the behaviour of animal aggregates (discussed in Chapter 5), and a theoretical model of the ways in which perceptual abilities interact with cognitive and movement abilities to generate aggregate properties (discussed in Chapter 6).

\subsection{Thesis overview}

The chapter directly following this introduction, Chapter 2, will provide a more thorough overview of existing animal aggregate research and discuss the current AR framework in more detail. The chapter will begin with a review of research findings on the biological underpinnings 
of aggregate behaviour, including a discussion of experimental techniques that have been developed to study aggregate-level behaviour. Chapter 2 will also discuss aggregate modelling, both mathematical and computer, with a focus on computer modelling techniques and species-specific aggregate models. Chapter 3 will begin with a discussion and critique of the AR framework, focusing on the issues with the AR framework in relation to creating animal aggregate models. Chapter 3 will then provide a detailed description of the PCA framework and discuss why it can overcome the limitations of the existing AR framework.

Moving from theory building to application of theory, Chapters 4, 5 and 6 will present the results of the three research projects- the $D$. rerio aggregate model (Chapter 4), the perceptual field effects model (Chapter 5) and the perception-cognition-action interaction model (Chapter 6). Chapter 4 will also include a description of the implementation of the PCA framework in the Breve simulation environment, and Chapter 5 will also include an investigation of the information requirements for aggregate cohesion. Chapter 6 will discuss animal aggregation in the context of complex systems. The thesis will conclude, in Chapter 7, with a general discussion of the results of the thesis, considering how the results of this thesis will affect current aggregate research, and providing an overview of future research to be undertaken based on the results of the thesis projects. 


\section{Chapter 2}

\section{Animal aggregate research: An overview with an emphasis on aggregate modelling}

This chapter begins by providing a general definition of animal aggregation and discusses properties of animal aggregates that are typically of interest to researchers. The chapter goes on to discuss research relating to the role of perception in aggregation and experimental methods for studying aggregate behaviour. The chapter then turns to a discussion of aggregate modelling, and the origin of the AR framework. Having summarised the AR framework, current aggregate models (both mathematical and computer based) are considered and the findings of these models reviewed.

\subsection{Definition and properties of animal aggregates}

Animal aggregate researchers frequently rely on our familiarity with common animal aggregates (e.g., bird flocks, cow herds) to provide an understanding of the phenomenon of animal aggregation, rather than providing an explicit definition. However, some attempts to define the

term 'animal aggregate' have been made. For example, speaking about aggregates in general, Toner and Tu (1998, p. 4828) define an aggregate as "the collective, coherent motion of large numbers of self-propelled organisms." Speaking specifically about fish, Hemmings (1966, p. 449) described a fish school as "a group of individuals in dynamic equilibrium, with the sensory 
input of each fish providing a feedback of information affecting the maintenance and behaviour of the school."

Since researchers generally assume that animal density is a key component of animal aggregates (see for example, Gerlotto and Paramo, 2003; Mogilner, Edelstein-Keshet, Bent L. and Spiros, 2003), a concrete, more spatially based definition of an animal aggregate could be given as: at a time $t$, a volume of space where the average density of the animals is high, surrounded by a volume of space where the average density of the animals is low, or zero (see Figure 1). This definition, similar to one provided by Camazine et al. (2001) is practical, due to its reliance on observable properties, but it does not acknowledge the importance of interactions and information exchange between individuals in determining the constituency and behaviour of the aggregate. An information-based definition of aggregation, motivated by the PCA framework, will be provided in Chapter 5 .

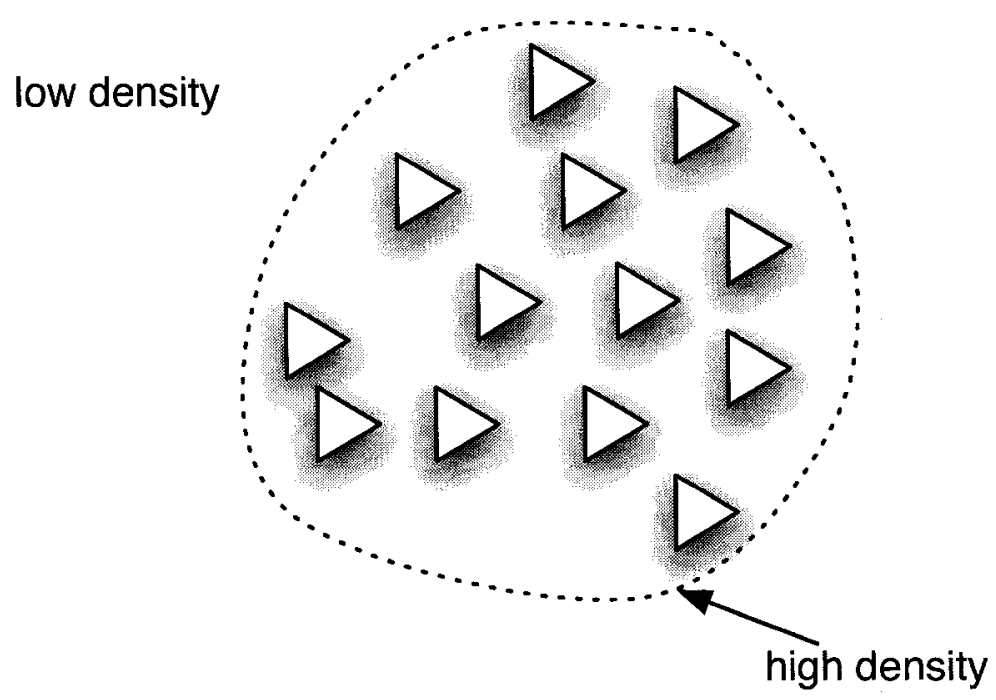

Figure 1: Using a simple density measure to define the concept of an animal aggregate.

In discussing aggregation, researchers may focus either on the behaviour and properties of individuals within the aggregate or on the behaviour and properties of the aggregate as a whole. This will be discussed in greater detail in Section 2.2.2 but a brief overview will be presented now in order to introduce concepts relevant to individual and group properties. In terms of individual behaviours (see Figure 2), researchers may observe and measure the 
orientation of the animal in relation to other animals within the aggregate and in relation to other items within their environment. Caraco and Bayham (1982), for example, considered the orientation of house sparrows, (Passer domesticus), feeding within house sparrow flocks relative to other sparrows and environmental disturbances. Researchers may also consider inter-individual spacing (the spacing of animals with respect to each other) and the movement patterns of particular individuals within the aggregate (using measures like individual velocity and individual turning angle). Miller and Stephen (1966), for example, determined that sandhill cranes (Grus canadensis) maintain a typical space of $5.8 \mathrm{ft}$ between individuals when aggregating. With respect to individual movement patterns, Ikawa and Okabe (1997) observed that while aggregating mosquitoes (Culex pipiens pallens Coquillet) remain grouped in a particular volume of space, individuals move through this space in an uncoordinated manner.

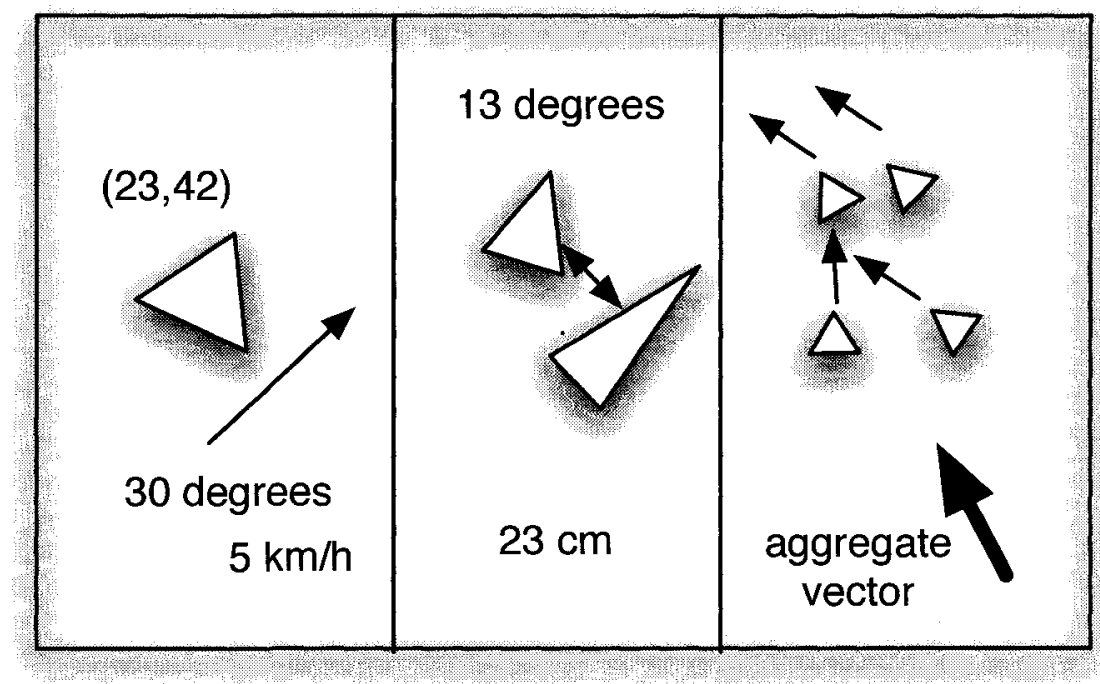

a

b

C

Figure 2: a) Measuring properties of an individual in an aggregate (orientation, velocity, position). b) Relational properties between individuals in an aggregate (angle and distance). c) Individual properties in the context of the aggregate as a whole (individual velocities relative to aggregate velocity).

With respect to the properties of the aggregate as a whole (see Figure 3), researchers may observe and analyse movement patterns of the entire aggregate, either by using measures like group velocity and group turning angle or by making more qualitative observations about movement patterns. For example, Graham, Pagès and Hamner (2001) observed that jellyfish 


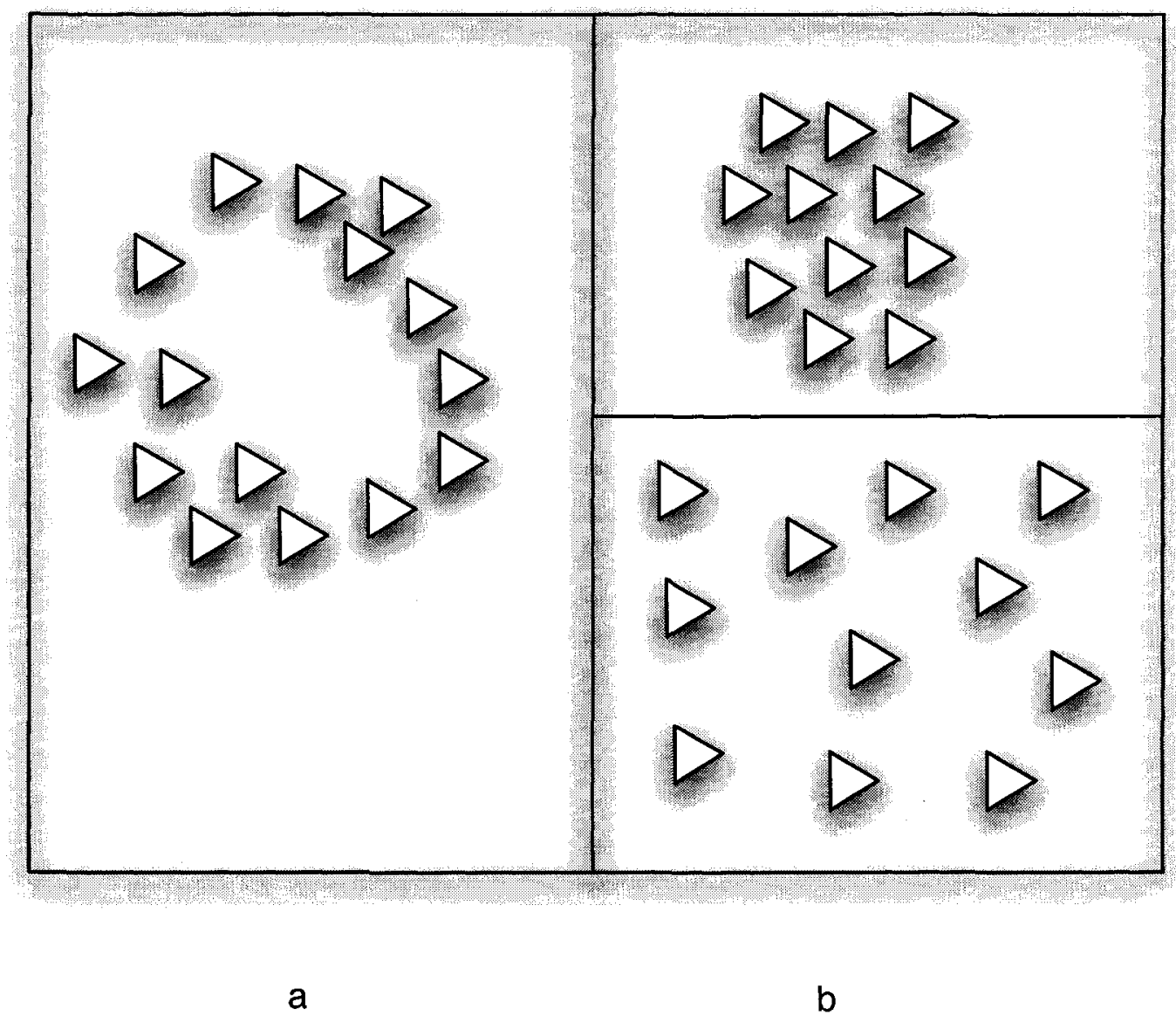

Figure 3: a) An aggregate with a toroidal aggregate shape. b) An aggregate changing from high to low density over time. 
aggregates (various species) regularly move up towards the surface during the day and down away from the surface at night. Researchers may also investigate the typical shapes formed by the aggregate. Soria, Bahri and Gerlotto (2003), for instance, investigated the effect that the approach of a survey vessel had on the school shapes of pelagic fish. They may also consider the average density or volume of the aggregate, using bounding boxes or by measuring the average distance of individuals in a group from the centre of the group. Carbone, Thompson, Zadorina and Rowcliffe (2003), for example, investigated the changing density and spatial patterns of feeding barnacle geese (Branta leucopsis) flocks over time. Lastly, researchers may consider the overall alignment of individuals in the aggregate with other individuals in the aggregate, using measures like group polarisation to indicate how aligned the group is, and group confusion to indicate how unaligned the group is. Aggregate measures used in this thesis are discussed in Chapter 4, Section 4.4.1.

\subsection{Species-specific aggregate behaviour}

\subsubsection{The individual underpinnings of aggregate behaviour}

The importance of attraction and repulsion Parr (1927) is typically cited as the first researcher to present a general theoretical description of aggregate behaviour. Observing chub mackerel (Scombrus colias) in the New York Aquarium, he concluded that for a fish, or other aggregating animal, to maintain its membership in an aggregate, this animal must successfully position itself over time so that it is neither too close nor too far away from the other animals in the aggregate. To maintain this balance over time, individuals within the aggregate must move towards other animals in the aggregate when they become too far away, and move away from other animals in the aggregate when they become too close (see Figure 4).

Parr referred to the behaviour of moving towards other animals as being attracted to them, and further noted that this attraction must be balanced by 'antagonistic stimulations' once a fish had reaches a certain proximity to its neighbours. He hypothesised that these two individual behaviours, repulsion from and attraction to the animals near them in the group, were sufficient to explain how animals moved together in groups. 

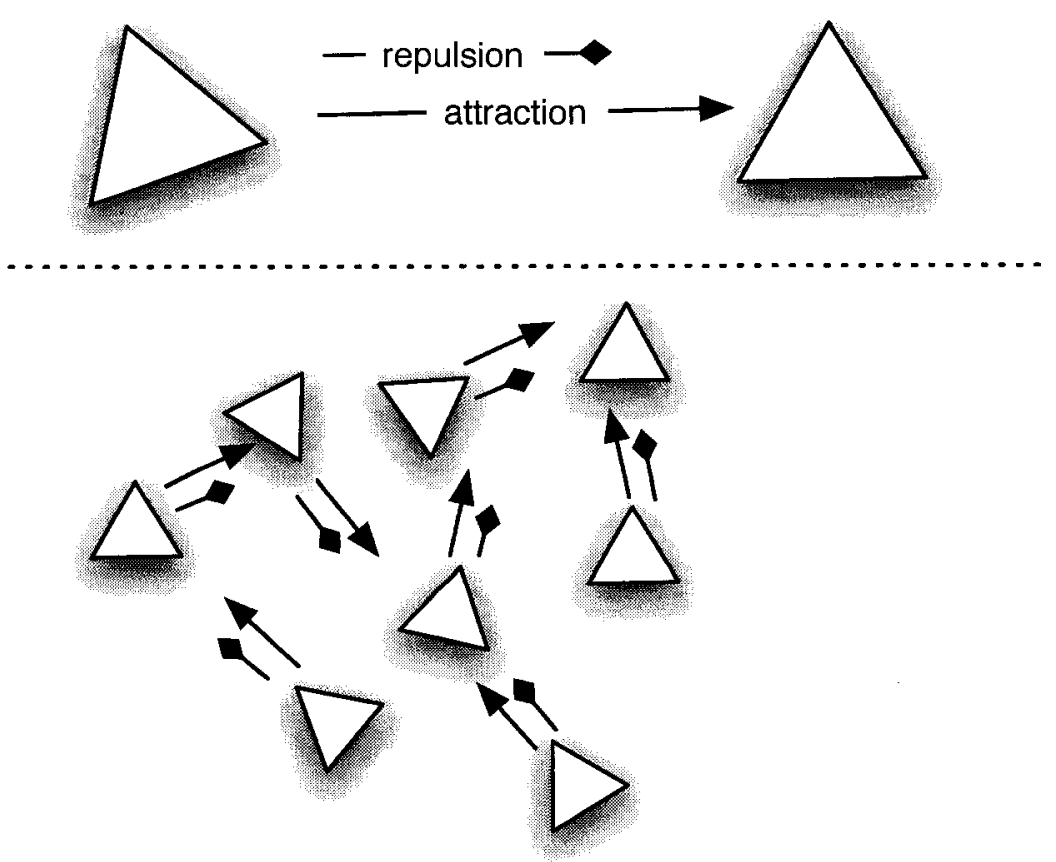

Figure 4: Organisms are both attracted to and repelled by other organisms. This combination of attractive and repulsive stimuli leads to aggregate behaviour.

The role of sensory abilities In addition to developing this attraction-repulsion characterisation of aggregate movement, Parr showed, in a very general way, that animals rely on particular sensory abilities to receive and process attractive and repulsive stimuli and thereby maintain their positions within aggregates. He demonstrated this aspect of aggregation by temporarily blinding a number of chub mackerel (Scombrus colias) using lampblack, and then returning these fish to the aquarium containing their original school. He observed that, while blinded, these fish remained isolated in the aquarium. Upon regaining their vision, however, they returned to their school and exhibited typical schooling behaviour once again. Parr concluded from this experiment that animals process information from particular senses when forming aggregates. He further noted that different species may rely on different sensory abilities to achieve aggregation.

The importance of specific types of sensation to aggregation has been further supported by more recent experiments that show that fish and other marine animals rely most specifically on visual perception and lateral lines for schooling. Partridge and Pitcher (1980), for example 
showed that saithe (Pollachius virens) with intact lateral lines but without vision could maintain their position in a school, as long as the school did not suddenly dissolve and reform as the result of a startle reaction. Fish with intact vision and no lateral lines could also school, but their position in relation to other fish in the school was affected by the loss of their lateral line perceptions.

The possible role of other perceptions in fish schooling has also been investigated. Hemmings (1966), for example, investigated the attraction of the roach fish (Rutilus rutilus) to visual cues vs. odour cues and found that, while roach were attracted both to the odour of other healthy roach in the water and to the physical (visual only) presence of other roach, they were more strongly attracted to the visual cues. Hemmings also showed that roaches were repelled by the odour of injured roaches.

Perceptual requirements for the reception of species-specific appropriate attraction and repulsion stimuli have also been investigated. Buskey (2000), for example, investigated the visual acuity of the mysid (Mysidium columbiae), a small aggregating crustacean, by having the mysids follow line patterns on a rotating drum, which they did without training. Buskey found that the majority of the mysids swam in the direction of the drum when stripes were $4 \mathrm{~mm}$ or larger in width, but that this number dropped as the width dropped until, at under $1 \mathrm{~mm}$ the percentage following the drum dropped to less than $60 \%$ of the school, suggesting that at this point the visual acuity, or resolution of the mysids was insufficient to allow for aggregating behaviour. Buskey concluded that this level of visual acuity was consistent with the survival requirements of the mysids, who had to follow other members of their aggregate at distances within the range of their visual acuity as measured by the experiment and also use their vision to locate tree roots of a certain minimum size for concealment from predators. The inclusion of perceptual acuity in aggregate models will be considered further in Chapter 3.

In some respects it seems almost trivial to acknowledge that animals use their sensory and perceptual abilities to successfully aggregate since, aside from scientifically unsupported theories involving mental telepathy (Selous, 1931) there are no other apparent aggregation mechanisms available to animals. This may explain the relative dearth of research into the precise role of sensation and perception mechanisms in aggregation. Researchers simply assume 
that aggregating animals are using one or more of their senses to aggregate, and move on to other questions about aggregation.

However, what is remarkable, in this context, is the fact that such a wide variety of animals, with a correspondingly wide variety of sensory and perceptual mechanisms, successfully aggregate. For example, although the sensory and perceptual mechanisms of Natterer's bats (Myotis nattereri) and guppies (Poecilia reticulata) differ quite widely, both of these animals form aggregates (Rivers, Butlin, and Altringham, 2006; Godin, Alfieri, Hoare and Sadowski, 2003). One of the challenges that remain for animal aggregate researchers is to determine how each of these diverse species use their particular perceptual abilities to form and maintain their aggregations, and then to produce models that are specific to these species.

\subsubsection{Aggregate-level findings, with a focus on research methods}

To some extent, it has only been recently that technology has developed to the point that detailed, quantifiable observations of the behaviour of animal aggregates can be made, thus opening up the possibility for more detailed and experimentally based aggregate research. Higher resolution digital imaging, combined with increased computing power makes it possible to track individuals within aggregates (Osborn,1997). With respect to aggregate properties as a whole, acoustic visualisation, again in combination with high-powered computing, allows for the study of large

aquatic animal aggregates in their natural ocean habitats (Greene and Wiebe, 1997). These data gathering techniques are complemented by data collection techniques and computational analysis techniques that allow for large amounts of data to be merged and analysed. (Jaffe, 1997; Ballerini, Cabibbo, Candelier, Cavagna, Cisbani, Giardina, Lecomte et al., (2008), Ballerini, Cabibbo, Candelier, Cavagna, Cisbani, Giardina et al., (2008)).

As discussed in Section 2.1, aggregate researchers are generally interested in a variety of group properties of aggregates (aggregate shape, inter-individual distance, aggregate density and aggregate movement) along with individual properties (animal velocity and movement decisions) that are believed to contribute to aggregate behaviour. In this section, a review of some of the representative species-specific research relating to each of these properties will be 
presented in the context of the research methodology used to gather data. Research carried out specifically in order to provide support for models of a particular aggregating species or group of species will be discussed in Section 2.5.

Observation and capture Simple visual observation is sometimes feasible when studying aggregates of smaller aquatic animals, and often entire aggregates can be captured in order to learn about the properties of individuals that make up the group. Using this technique, Spieler (2003) found that tadpoles of the frog Phyrynomantis microps increased their aggregation size when in ponds with relatively clear water, possibly in response to increased predation risk. Krause, Godin and Brown (1996) and Krause, Hoare, Croft, Lawrence, Ward, Ruxton, Godin and James (2000) observed and collected shoaling banded killifish (Fundulus diaphanus), golden shiners (Netomigonus crysoleucas) and white suckers (Catostomus commersoni). They found that shoals had a mixed composition with respect to species but that composition was still influenced by species and size. When shoals encountered each other there was transfer of individuals between shoals, as well as combination of shoals (fission and fusion phenomena).

Sonar and echosound Researchers have obtained a considerable amount of information on the size, shape and aggregate behaviour of fish schools using sonar and echosounder technology. Axelsen, Anker-Nilssen, Fossum, Kvamme and Nøttestad (2001) observed herring (Clupea harengus) being attacked by Atlantic puffins (Fratercula arctica) and recorded a wide variety of shape changes in response to these attacks, (including bend, hourglass, vacuole and split) along with density propagation through the school. Gerlotto, Bertrand, Bez, and Gutierrez (2006) observed anchovies (Engraulidae) using multibeam sonar and were able to record the propagation of a flash response (where fish rotate so that their silver side is visible) moving through the aggregate, which they speculate is a means of rapidly communicating predator attack through a large school. Pelagic fish schools (Soria, Bahri and Gerlotto, 2003) and clupeid fish schools (Gerlotto and Paramo, 2003) have also been observed using multibeam sonar and echosound, with findings that schools tend to be longer than they are wide, but that there is still considerable variation 
in school shape and behaviour, even between schools of the same species found in different locations.

Photography and film Photography and film have enhanced the ability of aggregate researchers to analyse and quantify their observations, particularly when the individuals in the aggregate can be easily distinguished and identified, either as a result of fortuitous natural circumstances or deliberate lab setup. Miller and Stephen (1966) used aerial photography to photograph Sandhill crane (Grus canadensis) flocks that were feeding in fields and found that the distance between cranes remained consistent, at $5.79 \pm 0.16 \mathrm{ft}$, regardless of group size. Carbone, Thompson, Zadorina and Rowcliffe (2003) used a similar method to monitor flock expansion in feeding barnacle geese (Branta leucopsis), finding that flocks expanded over feeding time. Caracoa and Bayham (1982) used Super-8 film to analyse the effects of temperature and sex of house sparrows (Passer domesticus) on flock size and nearest neighbour distance and determined that while sparrow sex did not have a noticeable effect on flock behaviour, lower temperatures led to an increase in flock size, and that nearest neighbour distance decreased as flock size increased.

Moving from naturalistic settings to lab studies, Krause and Tegeder (1994) were able to combine digitised and computer analysed video with a carefully designed experimental setup to determine that sticklebacks (Gasterosteus aculeatus) followed movement rules that minimised their approach time to a single conspecific when aggregating. Miller and Gerlai (2007) used a similar approach to study zebra danio (D. rerio) shoaling in the laboratory and found that baseline shoaling was altered by both the presence of food (increasing inter-individual distance) and predator presence (an initial increase in inter-individual distance followed by a more cohesive schooling behaviour).

Stereophotography and videography Stereophotography and videography, combined with digitisation and computer analysis provide what is potentially the most promising, although technologically most challenging, method for studying aggregate behaviour, particularly in naturalised settings. Aoki, Inagaki and Le Van Long (1986) used stereophotography combined 
with photo digitisation and computer analysis to study the three dimensional structure of schools of Jack Mackerel (Trachurus japonicus) and mackerel (Scomber sp.), determining nearest neighbour distances (1.43 body lengths (BL), $1.51 \mathrm{BL}$ ), individual fish size (20 cm, 14-23 $\mathrm{cm}$ ) and school density estimates (6.6 to $19.5 / \mathrm{m}^{3}$ for Jack Mackerel). More recently, Viscido, Parrish and Grünbaum (2004) used two video digital video cameras in a laboratory setting, combined with a calibration grid and video analysis software to determine the position and velocity of individual fish within small Devario aequipinnatus schools (see Chapter 4 for an in depth discussion of this methodology, which was also used to gather data on $D$. rerio movement for the $D$. rerio model discussed in Chapter 4).

The capabilities of stereo techniques for researching aggregates have recently been extended even further by Ballerini, Cabibbo, Candelier, Cavagna, Cisbani, Giardina, Lecomte et al., (2008) and Ballerini, Cabibbo, Candelier, Cavagna, Cisbani, Giardina et al., (2008), who used stereophotography combined with the trifocal technique (Hartley and Zisserman, 2003) and a variety of analysis techniques including statistical physics, image analysis and optimisation theory, to obtain data on the three-dimensional positions of individual European starlings (Sturnus vulgaris) moving within flocks over time (for flocks of up to 8000 starlings). Using this technique they were able to obtain quantitative measures for a variety of aggregate properties of starlings flocks, as well as properties of individual starlings within these flocks. With respect to aggregate properties, they found that, for the starling flocks they analysed, flocks had a typical shape (thin and long), distinct from size of flock and that flock density was not uniform, but instead was most dense on the periphery of the flock. With respect to individual properties, they found that individuals moved throughout the flock, rather than maintaining a single position within the flock. Using some data extrapolation techniques they have also proposed (2008) that individuals within the flock use a number of nearest neighbours to determine their position within the flock, rather than considering all of the starlings within a certain area. This finding will be discussed further in Chapter 5, in the context of model results from that chapter.

Limitations: Technological and theoretical Some of the research projects discussed herein particular those by Ballerini, Cabibbo, Candelier, Cavagna, Cisbani, Giardina et al., (2008)- 
have only recently become technologically feasible. Technological limitations cannot fully explain the relative lack of species-specific aggregate models up to this point, however, because technology sufficient for recording individual movement has been available for some time. Another contribution to this lack is the use of the AR framework, as used to describe and explain aggregate behaviour. As will be discussed in Section 2.3.2, one of the strengths of the AR framework from a theoretical perspective is its abstraction of the perceptual underpinnings of aggregate behaviour. However, this same abstraction means that the AR framework does not easily lend itself to the creation of verifiable models of particular aggregating species.This is because its physics centred characterisation of attractive and repulsive forces and its relatively simplistic representation of animal properties make it difficult to connect it to species-specific behaviour. In order for the AR framework to take advantage of technological advancements, it needs to return to a consideration of the perceptual and other biological underpinnings of aggregate behaviour as investigated by Parr (1927), Partridge and Pitcher (1980), and other researchers discussed in Section 2.2. This tension between specificity and abstraction will be considered more fully in Chapter 3.

\subsection{Modelling aggregation}

\subsubsection{Breder's model: Drawing an analogy with physical forces}

One of the challenges of modelling aggregates is the development of modelling techniques that are both computationally tractable and that can take into account the many interactions among individuals in an aggregate. Breder $(1951,1954,1976)$ was one of the first researchers to generate mathematically tractable models of the behaviour of schooling fish by drawing an analogy between fish in an aggregate and atoms held in a crystal lattice, and by comparing the responses to social stimuli of fish in an aggregate with the attractive and repulsive forces that exist between atoms. In Breder's 1954 model, aggregation depended simply on $a$, the attractive force between two individuals, $r$, the repelling force and $d$, the distance between the individuals, along with two constants, $m$ and $n$ (see Equation 1). These combine to provide $c$, a measure of the cohesiveness of the school as a whole. Breder then used Coulomb's law of magnetism and 
electrostatics, in conjunction with some simplifying assumptions, to derive Equation 2, where $r_{1}$ and $r_{2}$ are the repulsion of fish 1 (or school 1) and fish 2 (or school 2).

$$
\begin{aligned}
& c=a / d^{m}-r / d^{n} \\
& c=1-r_{1} r_{2} / d^{2}
\end{aligned}
$$

Breder's models began the practice of simplifying the stimulus and response component of aggregation by making use of a physical forces analogy. While acknowledging the importance of perception in actual aggregate behaviour, by positing the existence of social forces, analogous to physical forces, Breder and later modellers (e.g., Okubo 1986; Edelstein-Keshet 2001) were able to avoid modelling the stimulus response aspect of aggregation explicitly. This made models mathematically tractable and allowed modellers to focus instead on the more general question of how attraction and repulsion in individuals could lead to aggregation. This in turn led to the widespread incorporation of the concepts of attractive and repulsive social forces as a basic component of aggregate models, which in turn resulted in the adoption of the AR framework as the de facto framework for modelling aggregation.

Breder's model also demonstrates the way in which aggregate models generally start by considering individual properties but then switch to a focus on group properties. The variables a and $r$ reflect individual properties, but it is assumed that all individuals within the group share the same value for these properties. This allows a single value to be used for these properties, which, again, makes the model mathematically tractable and in turn allows conclusions to be drawn about the group property, $c$. Effectively, a more detailed representation of the heterogeneity and behavioural complexity of actual animals is avoided in order to obtain analytical power. Later models have also followed this pattern. Adioui, Treuil, and Arino (2003), for example, have developed a model that considers, as one of its main variables, a state variable that reflects the proportion of individuals in a particular unit of volume that have a certain orientation angle. The modellers then create a system of equations that relate to this state variable and explore the resulting properties of the mathematical model as they relate to group aggregate properties. 


\subsubsection{A typical aggregate model}

Breder's model is only one example of how the AR framework can be used to model aggregation. More generally, in modelling terms, the AR framework posits that each animal within an aggregate maintains the cohesion of the aggregate by following particular attraction and repulsion rules. Movement through space by an individual at a particular time, $t$, is determined by:

1. the attraction and repulsion equations (along with equation parameters) associated with individuals in the aggregate.

2. the inputs to the attraction and repulsion equations at that time, $t$, which themselves depend on the state of the environment at that time- in particular the position and velocity of other individuals in the environment.

3. the outputs of the attraction and repulsion equations, possibly combined with additional reaction equations, which determine how the position and velocity of the individual will change from time $t$ to time $t+1$ (see Figure 5).

Attraction and repulsion equations may vary in form, as long as they produce both attraction and repulsion. For example, Couzin, Krause, James, Ruxton and Franks (2002) define the attraction and repulsion behaviour of individuals as follows:

- If the distance between an individual, $i$, and other individuals, $j$, is less than a certain distance, $r$, then adjust the agent's direction of travel, as follows:

$$
\vec{d}_{i}(t+\Delta t)=-\sum_{j \neq i}^{n_{r}} \frac{\vec{c}_{j}(t)-\vec{c}_{i}(t)}{\left|\vec{c}_{j}(t)-\vec{c}_{i}(t)\right|}
$$

where $\vec{c}_{j}$ is the position vector of the neighbour at time $t$, and $n_{r}$ is the number of neighbours within the specified distance, $r$ at time $t$. This points the agent away from its neighbours, resulting in it moving away from them in the next time step. 

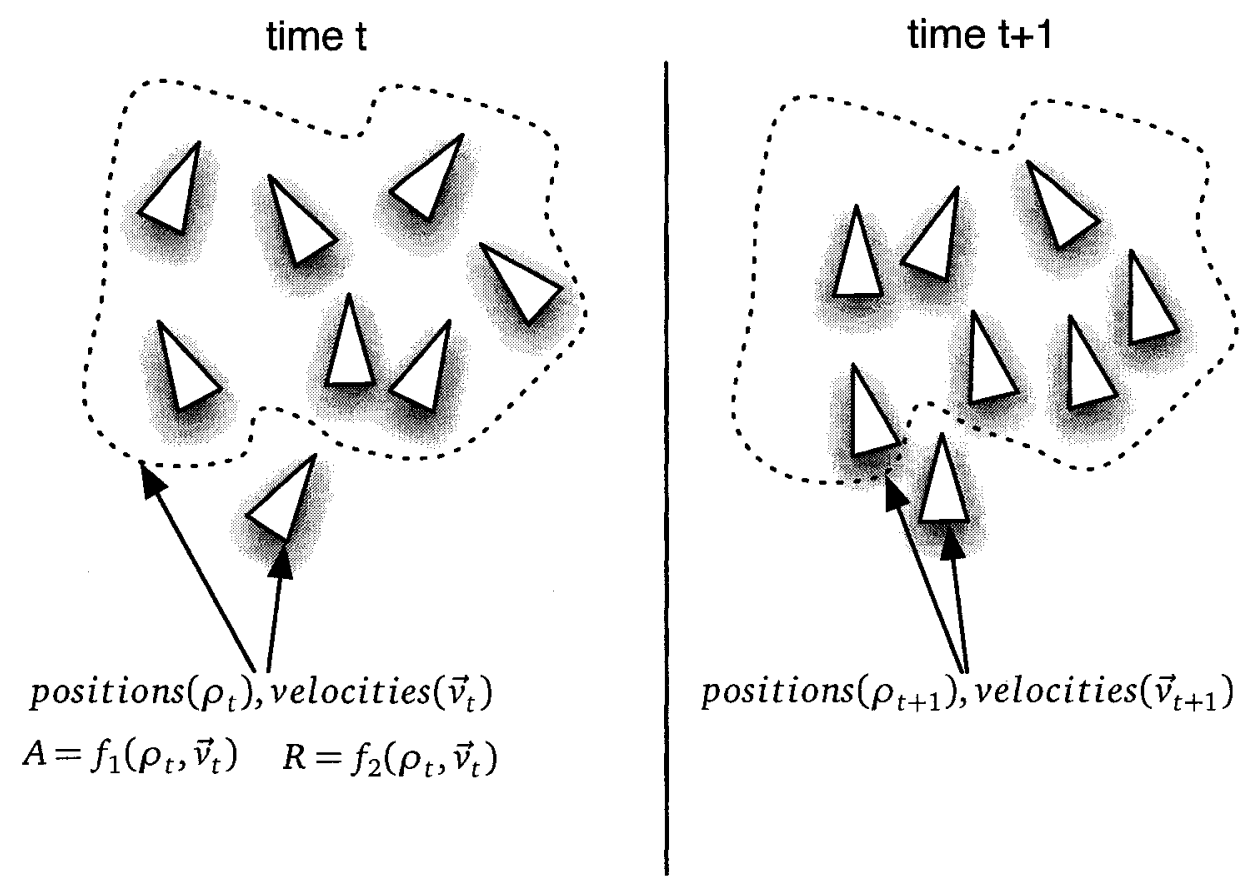

Figure 5: Calculating the behaviour of individuals within the aggregate over time. Each agent changes its position, $\rho_{t}$ and velocity, $\vec{v}_{t}$ based on the position and velocity of nearby agents, and the attraction and repulsion equations assigned to it.

- If this is not the case, then:

$$
\vec{d}_{i}(t+\Delta t)=\sum_{j \neq i}^{n_{a}} \frac{\vec{c}_{j}(t)-\vec{c}_{i}(t)}{\left|\vec{c}_{j}(t)-\vec{c}_{i}(t)\right|}+\sum_{j=1}^{n_{o}} \frac{\vec{v}_{j}(t)}{\left|\vec{v}_{j}(t)\right|}
$$

where $\vec{v}_{j}$ is the velocity of the neighbours, $n_{o}$ are the neighbours within a particular distance $o$ from the individual (but not closer than $r$ ) and $n_{a}$ are the neighbours that are within a particular distance, $a$ from the individual (but not closer than $o$ ), at time $t$. This results in the agent pointing towards its neighbours and matching its velocity with the velocity of the neighbours.

These two equations, when used to calculate the positions of each individual in the aggregate at each moment in time, result in aggregation behaviour by the group of agents over time. They are effectively force equations that describe the influence of the social forces exerted by other agents on a particular agent's position and velocity. As such, they act on agents, and determine the overall behaviour of the model. 
This abstraction away from biology towards the adoption of a more simplistic physical forces analogy is a major advantage of the AR framework. Because it does not specify any physical mechanisms of attraction and repulsion, but instead only assigns abstract, descriptive, attraction and repulsion rules that act on individuals within the aggregate, models using the AR framework are not tied to any particular aggregating species. Thus, the AR framework functions as a general theory, which can capture the essential aspects of aggregation across species. This, in turn, has allowed animal aggregate researchers to study the general properties of aggregation without the need to refer to the behaviour of particular animal aggregates in the wild.

In addition to this useful generalisability, the AR framework has two other major advantages, from a modelling perspective. The first is that generally only a few parameters are required by the framework, so that it is fairly simple to create models. The second is that the modelling concepts of attraction and repulsion can be concretely defined with equations, despite the fact that they are recognised to be only analogies for the actual mechanisms of aggregate behaviour. This means that there is little doubt as to how abstract models of aggregate behaviour are implemented, and model results can be clearly analysed, explained and compared. Having said this, because of its generalities, the AR framework has some difficulties describing animals in specific enough terms to capture relevant differences between animals. It also has difficulties connecting with particular natural scenarios (for example, transitions in aggregate behaviour when the temperature drops, or when the sun sets). Thus, although the AR framework excels at drawing theoretical conclusions about aggregation, it is often difficult to apply or relate these conclusions to aggregates in the natural world. This will be discussed further in Chapter 3.

\subsubsection{Recent developments in animal aggregate models}

Starting in the early 1990s, particle physicists looking for a new area in which to apply some of their modelling knowledge began to create models of animal aggregates by extending their existing particle physics models (see Section 2.4.1 for a detailed discussion of these models). At roughly the same time, computer scientists working in the field of distributed artificial intelligence began to develop modelling techniques, now referred to as multi-agent simulations 
(MAS), that would allow them to understand how individuals work together to produce group behaviours (Bond and Gasser, 1988) (see Section 2.4.2 for a detailed discussion of these models). MAS modelling techniques were quickly adopted by biological researchers (in particular ecologists and biologists studying population dynamics), who refer to these types of models as individually based, spatially explicit models (IBSEMs) or sometimes simply as individually based models (IBMs) (see Wiegand, Revilla, Knauer (2004) for a discussion of the role of IBSEMs in biology).

The IBSEM modelling methodology is particularly promising for animal aggregate researchers because it allows researchers to methodically change the behaviours of individuals within an aggregate model and observe how these changes affect the movement patterns of the aggregate as a whole. This, in turn, affords aggregate researchers greater insight into the ways in which the behaviours of individuals within an aggregate generate particular aggregate behaviours. In this respect, IBSEMs differ from traditional mathematical models of aggregation, which frequently avoid individual-level description and focus instead on describing aggregate behaviour using global variables like aggregate density or average aggregate velocity. They then analyse the change in these variables over time using differential equations (see, for example, Flierl, Grünbaum, Levin and Olson, 1999; Lutscher, 2002).

Mathematical models take this approach because their focus is on finding general, solvable solutions to aggregate equations for all values of variables. Consequently, most of these mathematical models of aggregates, whether developed by biologists or physicists, are not intended to reflect the behaviour of a particular aggregating species. Instead, their goal is to explore aggregation behaviour more generally. Researchers using IBSEMs, however, often do wish to claim that their models are predictive or explanatory of either the behaviour of a specific species (e.g., locusts (Schistocerca gregaria), Buhl, Sumpter, Couzin, Hale, Despland, Miller, and Simpson, 2006) or, more often, a group of species (e.g., schooling fish (Parrish et al., 2002) or ungulates (Turner, Wu, Romme and Wallace, 1993)). Thus it is important to consider the means by which these IBSEM modellers validate the claim that their model accurately reflects the behaviour of the particular aggregating species that they are modelling - an issue which is central to this thesis and which will also be taken up in detail in Chapter 3. 


\subsection{Current findings by aggregate models}

As discussed in Section 2.3.3, there are two main types of models that consider questions relating to aggregate behaviour- models that are mathematical in nature, which come to conclusions by solving or analysing mathematical equations, and IBSEMs, which are written as computer programs and have individual agents applying particular rules to determine how the aggregate as a whole will behave. Mathematical models of aggregation frequently consider more fundamental aggregate questions (e.g., what are the minimum requirements for the existence of coordinated movement) while IBSEMs frequently consider how varying the values of a particular parameter of interest (e.g., the values of attraction and repulsion) can influence the behaviour of an aggregate. IBSEMs are also frequently interested in the contribution the behaviours of individuals make and are more likely to make claims about the behaviours of particular animals and how they aggregate. Both types of models will be discussed in this section.

\subsubsection{Findings of mathematical models}

Vicsek, Czirók and others (Vicsek, Czirók, Ben-Jacob, Cohen and Shochet, 1995; Czirók, Stanley and Vicsek, 1997; Czirók and Vicsek, 2000) motivated a revival in aggregate modelling by mathematically describing and exploring the behaviour of what they called interacting selfpropelled particles. These were zero mass particles that moved with a constant velocity and set their own alignment based on the alignment of local neighbours where what constitutes a local neighbour is determined by some chosen function, $S$. Czirók and Vicsek (2000) give two examples of $S$, one a circle with radius $r$, and one a number of nearest neighbours on a lattice. A noise function was also introduced in the model, so that particles could not align perfectly, and equations developed that connected alignment to particle density. Czirók and Vicsek (2000) found that, even in this simple model, aligned moving clumps of self propelled particles formed for some values of particle density and noise.

Toner and Tu (1998), motivated by Vicsek et al. (1995), used a mathematical model to consider how aggregates that begin without cohesive movement in a particular direction are able to break this symmetry and begin moving in a particular direction. They found that over 
time high and low density fluctuations build up within the aggregate, which in turn lead to a spontaneous breaking of symmetry and group movement in a particular direction. Aldana and Huepe (2003) also considered an extension of Vicsek's simple particle model, where particles could interact via direct random connections instead of only local connections. They referred to this variant as a vectorial network model. They found that even when interactions were determined randomly (analogous to moving at infinite velocity), there were still transitions between ordered and disordered states, simply as a result of there being long-range interactions of some kind.

Building on these more general findings, a variety of mathematical models explored the implications of varying attraction and repulsion equations and coefficients for aggregate behaviour. Grégoire, Chaté and Tu (2003) developed a model that was an extension of Vicsek et al.'s (1995) simple particle model by adding attraction and repulsion forces (described as body forces) in addition to the original alignment force. They added weights to the alignment, attraction and repulsion parts of their equations and found that, depending on the strength of the alignment force as compared to the attraction and repulsion force, the aggregate moved through phases which they compared to the gas, liquid and solid phases of a physical material. For weak body forces, the aggregate did not remain cohesive and was in a gas-like state. For stronger body forces, the aggregate behaved in a manner analogous to a liquid or solid. Strength of the alignment forces then determined whether or not this liquid/solid was stationary or moved through space.

In an extension of an earlier model by Grünbaum (1998), Adioui, Treuil et al. (2003) and Adioui, Arino, Smith and Treuil (2003) considered the affect of alignment and diffusion with respect to aggregate behaviour. Their models incorporated an approximation of attraction and repulsion (what they refer to as an orientation analogue of attraction and repulsion) by assuming that fish would turn left or right depending on where the majority of other individuals already in the group were pointing, but that this would be offset by a Fickian dispersion mechanism. They found that the extent to which the aggregate as a whole aligned depended on the ratio between the diffusion force and individual alignment tendencies, such that the ability of the aggregate as 
a whole to align depended on a certain minimum ratio of alignment and diffusion and that as the ratio increased aggregate alignment also increased, and time required to align decreased.

Lutscher (2003) used a mathematical, one-dimensional AR model to analyse how attraction and repulsion could lead to grouping of individuals over time when the model began in a homogeneous state. Agent influence was local and a variable was included in the model equations to represent a 'radius of perception', which served to limit the influence of group density on the individual. Lutscher found that this AR model could produce both stable and unstable states, depending on how individuals reacted to a departure from their preferred density in the surrounding area, and that areas of high and low density could emerge from homogeneous states.

Modelling attraction and repulsion forces explicitly, and also using a one-dimensional model (but mathematically extending their findings into higher dimensions) Mogilner et al. (2003) explored how changing values of $A$ and $R$ - the strength of attraction and repulsion force in these areas, along with values of $a$ and $r$ - the radius of attraction and repulsion - affected the behaviour of an aggregate. In this model, agents could be influenced by all individuals in the group, but the farther an individual was from an agent, the less their influence on the agent was. Mogilner et al. found that in order for well spaced, globally stable aggregation to occur, it was necessary that the force of attraction be weaker than the force of repulsion, and the range of attraction had to be greater than the range of repulsion, although this was not also sufficient for aggregation in all circumstances. They also found that the distance between individuals within a stable group was smaller than that of the preferred distance of an individual with respect to other individuals.

Thus the general finding of these mathematical models was that the behaviour of an aggregate - in particular its level of alignment and cohesion - can be significantly altered simply by altering the weights and equations associated with individual attraction, repulsion and alignment behaviours. Specifically, by altering attraction and repulsion equations, the behaviour of the group may range from disordered and stationary, to aligned and moving through space, to remaining in an ordered but stationary state. This suggests that, within a particular aggregating 
group of animals, individuals in the group may control behaviour at the aggregate level by varying their individual attraction and repulsion behaviours in a coordinated manner.

However, as can be seen from the research presented here, the representations of animals in these models are highly abstract (often represented as zero dimension points moving along a line, in one-dimensional models), and the findings of these models are also abstract. Thus, although findings of these models are relevant with respect to what is possible in aggregates at a very theoretical level, it is not clear how these findings relate to actual aggregate behaviour, because representations of aggregating animals in these models are so simplified that it is very difficult to relate them clearly and directly to actual animals. Part of the reason for this simplification is that mathematical equations quickly become intractable when significant abstractions are not made. Computer-based models, which represent individuals directly, avoid this problem by simplifying and increasing the number of equations involved and then transferring the increased computational load to the computer. These type of models will be discussed in the next section.

\subsubsection{Findings of individually based, spatial explicit computer models (IBSEMs)}

To some extent, IBSEMs are automatically less abstract than mathematical models because they are composed of discrete agent objects that follow assigned rules. As a result of this representation of individuals, there is a subtle shift away from viewing agents merely as objects upon which social forces act, towards viewing them as actors that react to their environment, although the term 'social forces' is still generally used to describe attraction and repulsion. As a result, there is a more tangible connection between the agent and the animal and the agent rules and the behaviour of the animal. As well, because IBSEMs represent individual animals explicitly, IBSEMs can more clearly examine how individual properties contribute to group properties, with a focus on exploring the parameter space of the model to establish cause and effect relationships between individual and group properties. And finally, again as result of representing individuals explicitly, natural variations in animal properties are easily accommodated and stochastic behaviours are supported. 
Generally speaking, in aggregate IBSEMs, all agents follow the same assigned rules, but the specific instantiation of these general rules depends on the situation of the agent at time $t$. As well, for each agent, different rules often operate on different areas or volumes of space, relative to the position of the agent, with modellers referring to these areas or volumes of space as zones, ranges or areas (see for example Couzin et al. 2002, Huth and Wissel, 1992. See also Figure 6). Within this general format, IBSEMs may be two or three dimensional and may also be entirely abstract models, like the mathematical models discussed above, or models that incorporate experimental data and model specific aggregating species. All of these model types will be discussed below.

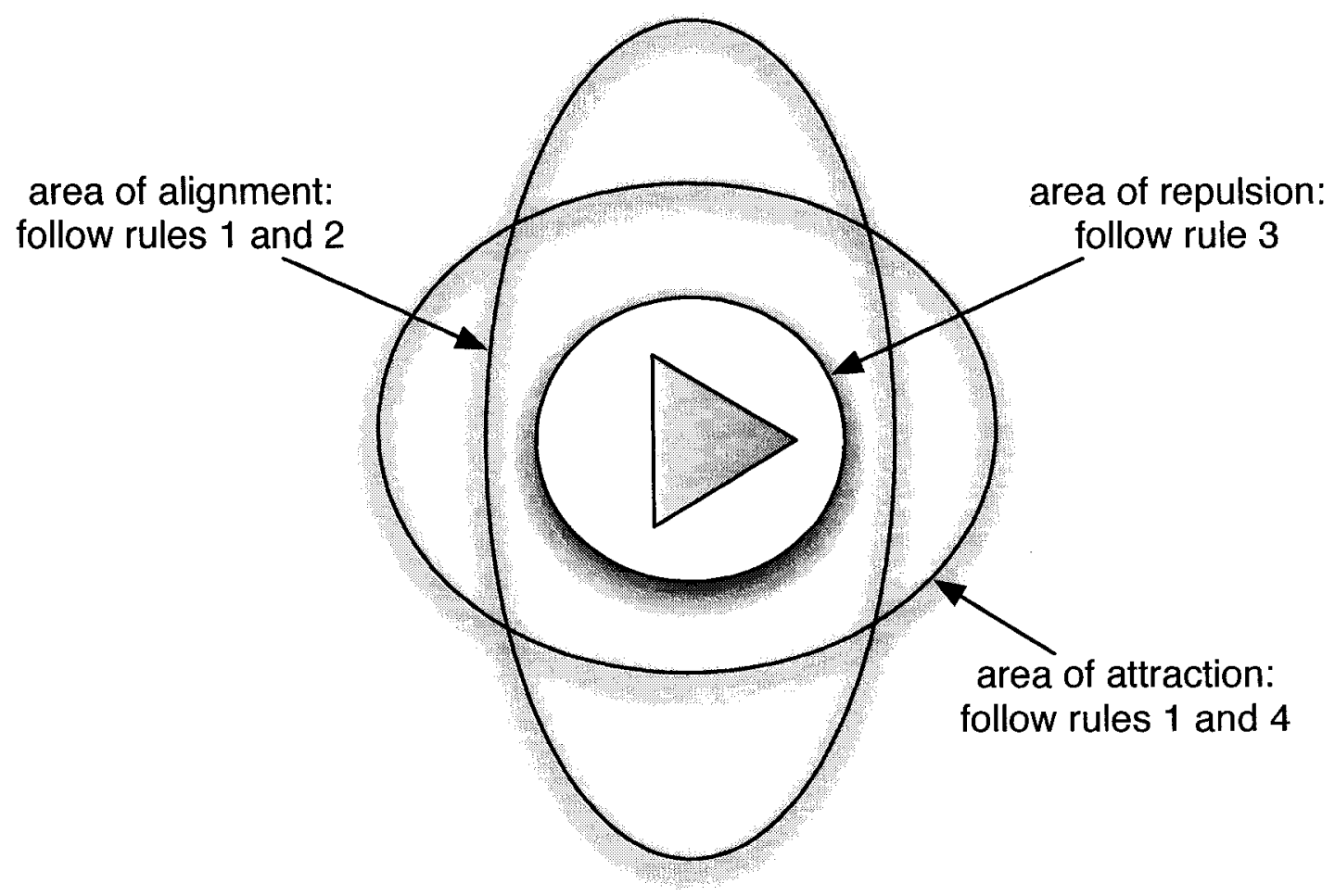

Figure 6: Individual rules, operating with particular zones of behaviour.

Abstract computer models, two-dimensional Two-dimensional models assume that some aspects of aggregate behaviour can be accurately modelled on a two-dimensional plane. This seems particularly plausible for aggregates like ungulate herds, fish swimming in shallow water and insects moving on the surface of the land or water. However, like the mathematical models 
discussed above, many two-dimensional models are not interested in deriving particular conclusions about specific aggregates but instead are interested in deriving more general conclusions about aggregate mechanics. An early two-dimensional simulation model created by Aoki (1982), for example, explores in a very general manner how unified group movements can occur even in the absence of a specific leader and global knowledge of the group by individuals within the group. Multiple combinations of model parameter values were run for the model in order to explore the parameter space of the model, with the finding that model results were consistent across changes in group size but that changing behavioural rules (e.g., removing agents' alignment rule) resulted in changed movement patterns for the aggregate as a whole.

Warburton and Lazarus (1991) used a two-dimensional model of between 2 and 9 agents to consider how attractive and repulsive forces of individuals in the model lead to common inter-individual distance in the group and group stability. By varying parameters associated with attraction and repulsion equations they were able to determine that the shape of these equations (i.e., linear, inverse sigmoid, convex, concave) influenced the average inter-individual distance in the group and that for 4 of the 8 functions considered, similar to findings by Mogilner et al. (2003), actual inter-individual distance was smaller than the preferred distance used by individuals. They also considered how the number of nearest neighbours influenced by an individual might interact with the social force equations. In this model, group size was small and all agents could be influenced by all other agents but did not necessarily use information about all agents when determining movement. They found that only a single influential neighbour was required for aggregate cohesion under some circumstances- specifically when there was no random vector (referred to as a 'searching vector') included in the movement rules- but that when the searching vector was included a minimum number of neighbours that was close to half the group size was required for group cohesion.

Huth and Wissel (1992) also considered how aggregation is influenced by the decision rules individual agents use to react to the location of other local agents. In particular they considered how different turning angle algorithms influence aggregation by analysing the effects when a fish agent either determined its turning angle by considering a particular number of its neighbours but then responding only to the 'best' one, as defined by its angle from the front of the agent 
(the D-model) or took all neighbours into account in determining its response (the A-model). They found that averaging across all neighbours (the A-model) led to a faster moving, more oriented group, although both groups remained cohesive, as measured by the expanse of the aggregate. Reducing the maximum turning angle improved the behaviour of the D-models. Huth and Wissel also varied the radius of the areas of attraction, repulsion and orientation in order to evaluate their effects on model behaviour and found that the larger the orientation range, the more aligned but less cohesive the aggregate. It is interesting to note that in the process they state that these areas represent 'sense capabilities' and suggest that they differ for different species. However, they do not distinguish between the attraction and repulsion forces acting in these areas and the perceptions themselves.

Considering aggregation in a larger environmental context, Zheng, Kashimiori, Hoshino, Fujita and Kambara (2005) investigated how the presence of predators might affect the behaviour of individual fish in schools, and how this, in turn might affect the behaviour of the entire aggregate. They modelled this by varying the behaviour rules fish agents chose to follow when they received information about a predator. In some cases the fish agent responded only to the predator, while in other cases the fish agent also took into consideration the presence of the other fish in the aggregate when deciding how to move. They found that optimum predator evasion occurred when fish divided their behaviour between schooling and evasion, and when they had some tolerance for the presence of the predator, rather than immediately switching to an evasion behaviour.

Abstract computer models, three-dimensional Three-dimensional models offer researchers the chance to incorporate more realistic physical interactions and movement rules into models, particularly for animals like birds, fish and flying insects, which can move in three dimensions. Parrish et al. (2002) used a three dimensional model to consider how varying attraction and repulsion rules, as well as movement algorithms, influenced fish aggregate behaviour. They based several of their modelling parameters (e.g., fish speed and acceleration, fish visual blind spots) on qualitative data on fish movement and physiology, in particular, data gathered on giant danio (Danio aequipinatus) behaviour, making the model more explicitly grounded from an empirical 
perspective. Similarly to Warburton and Lazarus (1991), they found that varying attraction and repulsion equations caused corresponding variability across most aggregate measures, with the exception of aggregate alignment. They also found that number of neighbours and scaling functions applied to neighbour influence affected size and cohesion of the aggregate, as well as polarity and speed of the group.

Viscido, Parrish and Grünbaum $(2005,2007)$ used a similar three dimensional model to consider how population size and number of nearest neighbours used by individuals to determine movement could influence aggregate properties (2005) and how the functional form of a variety of forces applied to the individual (social forces including alignment, attraction, repulsion and neighbour influence, as well as random forces and frictional forces) affected aggregate behaviour (2007). They found that group properties are influenced by overall size of population and number of neighbours considered by individuals, and that, in general, all forces, with the exception of the random force, significantly influence aggregate behaviour. They also found specific patterns of influence for specific force forms. For example, they determined that a strong repulsion force was required (similar to the results of Mogilner et al. (2003)) for aggregation to occur and also that speed of motion, weighting of neighbours considered, and alignment impulses influenced both aggregate structure and the ability of the aggregate to maintain structure over time. The influence of individual properties on aggregate cohesion and other aggregate-level properties will be further considered in Chapter 6 .

In general, abstract IBSEM models of aggregates have been able to explicitly explore the behaviour of the aggregate with respect to how individual behaviours lead to group behaviours, with an emphasis on how particular attraction and repulsion rules of individuals determine model behaviour across parameter sets. Abstract IBSEM models have also been able to draw some more detailed conclusions about the effects of different movement rules on aggregationrules which are relatively straightforward to define and change in IBSEM models. However, they are generally unconcerned with relating their findings directly or concretely to actual animal aggregates. To achieve this, models must incorporate empirical information pertaining to actual animal aggregates, as will be discussed below. 
Models incorporating observable properties of individuals All of the models discussed to this point consider how either unobservable abstractions of individual properties (values of attraction and repulsion forces) or difficult to observe individual properties (behavioural reaction algorithms for responding to observed individuals) might influence aggregate behaviour. Many fewer models discuss results that relate more directly to observable animal properties (e.g., body size, movement capabilities or perceptual abilities). However, as computer simulations have become more sophisticated, a number of IBSEMs have been produced that attempt to determine how some of these observable properties influence aggregation. These models, in order to answer questions relating to physical properties of animals, have started to include more physically realistic perception and movement rules, as well as more realistic, fully embodied agents.

With respect to agent embodiment, Reynolds (1987) was arguably the first to create fully embodied aggregating agents, with multi-component, articulated three dimensional bodies, but his simulation was intended for animation rather than research. With respect to research on the behaviour of embodied agents in an aggregate, Brogan and Hodgins (1997) created an aggregate model consisting of two groups of dynamically simulated, physically realised agents, replicating one legged robots and bicycle riders (physically simulating both the bicycle and the rider), along with a control group consisting of point mass agents. They used this model to explore how movement dynamics influence aggregate behaviour. Agents considered their $n$ nearest neighbours when calculating movement (with $n$ varying between $1 / 3$ and all of the group) and attempted to move to a position based on the weighted location of these neighbours (with movement being influenced by body dynamics). Brogan and Hodgins found that the point mass version of the model was most successful at moving tightly together while avoiding obstacles, while the one-legged robot group required more spacing between individuals to avoid collision, and the bicycles had a difficult time avoiding collisions when turning.

In terms of being embodied in the sense of responding to environmental conditions in a physically realistic manner, Flierl, Grünbaum, Levin and Olson (1999) used a combined mathematical and individual based approach to examine how social aggregate forces interact with environmental factors to influence aggregate behaviour of aquatic organisms. They began 
by considering what aggregate affects can arise simply as a result of environmental conditions, and then added social forces to the model to consider how these would interact with the environmental conditions being modelled. With respect to these later models they found that although high amounts of turbulence can impede aggregation, low amounts of turbulence can enhance aggregation by providing more opportunities for agents to encounter each other, at which point social forces lead to the formation of aggregates.

Couzin et al. (2002) considered how two observable properties of individuals - turning rate and speed of individuals - influence aggregate behaviour, along with the influence of the spatial range over which agents receive information and population size. Their model included heterogeneity across these variables to reflect heterogeneity in actual populations. They found that aggregate properties were influenced by attraction and repulsion values, neighbour selection and movement algorithms. With respect to movement algorithms they varied speed and turning rate among individuals within the aggregate and determined that faster individuals remained within the aggregate but moved to the front of the aggregate. Individuals with a higher turning rate, however, tended to be positioned nearer to the centre of the group.

Viscido, Miller and Wethey (2002) considered how the manner in which agents decide to move, based on the information available to them, influences aggregate behaviour. They consider 4 different movement rules - random movement, simple nearest neighbour (similar to the decision rule considered by Huth and Wissel (1992)) a Hamiltonian rule (which required agents to consider their nearest neighbour) and the nearest neighbour of their nearest neighbour, and a collection of rules which they refer to as the Local Crowded Horizon rule. Although most of these rules refer to cognitive variables, this last rule could be considered to be a perceptual rule. For the Local Crowded Horizon movement rule, the influence of other agents was mediated by what they refer to as a 'perception function', so that animals farther away have less influence on the behaviour of the animal, because they are smaller and harder to see. They tested 6 different variants of this perception function to see how it influenced aggregate behaviour. In their conclusions they note that changing the perceptual function strongly influenced the behaviour of the aggregate, as did the other movement rules, and that all rules produced results that differed statistically significantly from each other, based on the aggregate measures used. 
Also relating to animal perception, Ward, Gobet and Kendall (2001) developed a twodimensional evolving aggregate model using artificial neural networks. In this model, agents detected other agents in two different regions of space, inspired by a distinction between the lateral line and visual perceptions of fish. Weights associated with each of these fields could change so that agents could choose which agent information to use in their movement rules (where agents included predators and food as well as other aggregating agents). Movement rules were simply 'turn left' or 'turn right', with constant speed. Predators and food (along with an energy measure) were introduced to the modelling environment to provide selection pressure and model weights evolved over time. Ward et al. found that although aggregation was not explicitly programmed into agents at the start of the simulation, with each successive generation, prey agents moved closer together, with respect to average distance from other prey agents. They also showed, through an analysis of weights on the neural nets of individual agents, that agents valued staying close (schooling) more than eating, and that the field based on vision was used to turn towards food while the field based on the lateral line was used to avoid predators.

Kunz and Hemelrijk (2003) constructed a two dimensional model that considered the effects of body size on aggregate behaviour. Fish were modelled as lines of two sizes (big and small), in addition to being modelled as points. Both vision and lateral line perceptions were explicitly modelled, and the shape of the area for which the fish received information was influenced by its body shape. The visual field for line agents was spherical, but the lateral line field was elliptical based on the argument that actual lateral line sensors would result in an elliptical information field. As well, attraction and repulsion equations were different for fish of different sizes, since larger fish would be expected to have longer lateral line fields. Kunz and Hemelrjik found that larger agents had larger inter-individual distances, that aggregate homogeneity was reduced when agent bodies were lines rather than points, and that elliptical fields resulted in more frequent turning by agents within the aggregate and smaller aggregates over all.

Hemelrijk and Hildenbrandt (2008) followed this two dimensional model with a three dimensional model created specifically in order to take into account criticisms, like those by Parrish and Viscido (2005), that some of the movement and perceptual rules used in the earlier model (Kunz and Hemelrijk, 2003) were unrealistic, in addition to school size being too small. 
In this model, agents moved through a three dimensional space at a preferred speed, but could change this speed in response to information about neighbouring fish. Agents had a blind zone that represented the visual blind spot of actual fish. The range of agent detection was decreased as density increased, to take into account the fact that at higher densities some fish are occluded by other fish. Agents followed attraction and repulsion rules, with each rule operating only in a particular volume of space. Hemelrjik and Hildenbrandt found that model aggregates were oblong with a higher density at the front than the back of the school, a group phenomenon that occurred in the model as a result of individual fish avoiding collisions while at the same time trying to maintain desired distances between themselves and neighbouring fish.

\subsection{Species-specific models of aggregation}

This thesis is chiefly concerned with creating a modelling framework that can be used to create species-specific aggregation models. There have been relatively few species-specific models of aggregation, arguably due to the limitations of both technology and the AR framework. Nonetheless some researchers have made considerable efforts to generate species-specific models based at least in part on experimental data about their species of interest. As well, some modellers, although referring to their models in a more generic way (for example as fish models) have based their models on experimental data gathered about the behaviour of specific aggregating species. Lastly, other models, while general enough to be easily applied to a variety of aggregating species, are species-specific in the sense that they have been created in order to provide their creators with insight into species-specific behaviours of interest. All three types of models will be reviewed in this section.

It is not uncommon for model creation to be motivated by observations of species-specific behaviour, but for the model itself to be a more general model of animal aggregation. A good example of this is the model by Hoare, Couzin, Godin and Krause (2004), which is motivated by the observed behaviour of banded killifish (Fundulus diaphanus). Although the model itself is a generic aggregation model with no species-specific characteristics, Hoare et al. used it to further their understanding of the way in which interaction distance influenced the size of banded 
killifish aggregates under various circumstances. Similarly, a model (Stöcker 1999) inspired by tuna behaviour, although not informed by experimental measures of tuna behaviour, allowed the researcher to gain an increased understanding of the way in which the energetics of school formation might influence maximum group size in fish, in order to better estimate tuna fish stocks.

In other cases, researchers wish to model a specific aggregate behaviour of a particular species and base at least one of their model parameters on experimental data gathered about either the individual behaviour or group behaviour of the species in question. Movement rates are relatively easily observed so this experimental data is most frequently incorporated. Dagorn and Fréon (1999), for example, were motivated by the tendency of tuna (sp. Thunnus) to gather under floating objects (generically referred to as 'logs') to create a two-dimensional model of tuna aggregation in the presence of logs. Swimming speeds of tuna in the model $(3.5 \mathrm{~km} / \mathrm{h})$ were based on experimental data and size of the model and position and frequency of logs were also based on experimental data. Several recent models of fish aggregation, already discussed in Section 2.4.2 (Parrish et al. (2002), Viscido et al. (2005, 2007), have also incorporated experimental data on fish movement of particular species in their models. Parrish et al. (2002) and Viscido et al. $(2005,2007)$ base the movement parameters (agent speed and agent velocity) for their agents on Danio aequipinatus movement gathered using Tracker 3D, a two camera tracking system. (This movement tracking system is used in this thesis to gather data on D. rerio movement and will be further discussed in Chapter 4).

In other cases, the relevant experimental data is behavioural rather than movement based. in their two-dimensional cockroach larvae model, Jeanson, Rivault, Deneubourg, Blanco, Fournier, Jost and Theraulaz (2005) based probabilities of cockroach larvae (Blatella germanica) behaviour (stopping, starting moving after a collision, starting moving in the absence of a collision and exiting the aggregate) on detailed laboratory observations of cockroach larvae in a large circular arena. They found that chance encounters arising out of an initial uniform distribution of cockroach larvae led to eventual large scale aggregation as a result of a process of gradual cluster amplification. In other cases the behavioural data is more general. Two models of Daphnia (Daphnia sp.) behaviour (Mach and Schweitzer 2007; Vollmer, Vegh, Lange and Eckhardt 2006) 
based the behaviour of agents in their models on the known attraction of daphnia to light sources, along with experimental data gathered on movement abilities (self propulsion and movement rate) and possible avoidance behaviours of Daphnia.

These models reveal that it is possible to base model parameters and agent behaviours on experimental data, an important step in the creation of models that can be connected to specific species, and a generally desirable feature of scientific models. At the same time, it is important to note that, although these models are at least to some extent created based on experimental data, models are much less frequently validated by experimental data - in particular, by comparing aggregate-level model behaviour with experimental data. As well, models are rarely used to make predictions of novel behaviour within actual aggregates but instead tend to be used to explain existing behaviour. It is also worth noting that although movement and behaviour data are incorporated into these models, perceptual data is much less frequently incorporated into species-specific models. In those cases where it is considered at all, as in Parrish et al. (2002), data frequently relate to more general across-species findings and potential effects of perceptual factors specific to the particular species of interest are not explored.

\subsection{Chapter summary}

A wide variety of research is relevant to animal aggregation, due to its complex and difficult to observe nature. This chapter has reviewed key findings in several areas. The chapter began by considering experimental findings relevant to the role of perceptual abilities in aggregation, and continued its experimental review with a discussion of experimental findings on aggregate properties, with an emphasis on the research methods that enabled these findings. Mathematical and computer based (simulation) models of aggregation were then considered, with attention focused on findings related to unobservable individual properties like attraction and repulsion, as well as more observable properties, like animal shape and speed. Lastly, aggregate models of specific species were reviewed, with attention to how these models were supported or verified using experimental results. 
Although these models have increased researchers' awareness of what is possible with respect to aggregation, they have been less successful at answering questions about what is actually occurring in real world aggregates. In particular, they do not answers questions about the mechanisms that specific species are using to aggregate, or why some aggregate-level phenomena are similar in widely differing species, while other aggregate-level phenomena can vary widely, even within the same species, from moment to moment. In the next chapter, issues with the modelling methodology described here will be discussed, along with the PCA framework, a new modelling framework that addresses and resolves many of the difficulties preventing the AR framework from being used to make species-specific models. 


\section{Chapter 3}

\section{Issues with the Attraction Repulsion framework and a proposed solution - the Perception Cognition Action framework}

This chapter begins by considering some shortcomings of the AR framework that become apparent when it is used to generate species-specific models of aggregation. To illustrate this, a general description of how a model of a particular animal aggregate might be constructed using the AR framework is provided, leading to a discussion of both practical and theoretical problems associated with using the AR framework to construct aggregation models. The chapter then discusses the role of information processing in aggregation and the ways in which perception-influenced aspects of information processing are tacitly incorporated into existing attraction-repulsion models.

The second half of the chapter, starting in section 3.3, presents the PCA framework. This framework, although compatible with the existing AR framework, is conceptually detailed enough to allow researchers to model specific animal aggregates. This part of the chapter will begin by discussing the relation between the AR framework and the information processing paradigm used in cognitive science. The chapter will then go on to describe the PCA framework, which connects the AR framework to the information processing paradigm. The chapter concludes by discussing how the PCA framework will allow modellers to avoid problems currently facing models generated by the AR framework, as detailed in section 3.1.2. 


\subsection{Methodological issues with the AR framework}

Particularly with the introduction of IBSEMs, modellers more and more frequently wish to move away from making very general claims about the theoretical underpinnings of aggregate behaviour towards making more specific claims about how aggregation actual works in the context of specific biological systems (e.g., Viscido et al. 2005; Hemelrjik and Hildenbrandt, 2008; Gueron and Levin, 1993). However, the AR framework, although well suited for exploring general aggregate theory, is less easily applied in these more biologically specific scenarios.

\subsubsection{Model Construction using the AR framework}

As discussed previously, the AR framework describes individuals in an aggregate as being acted on by social forces that cause them to be attracted to and repulsed by the individuals around them. Each individual's particular combination of reactions to these forces leads to the behaviour of the aggregate as a whole. Supplying the model with different attraction and repulsion rulesfor example, changing the distances at which individuals begin and cease to be attracted or repulsed, or the number of agents it uses to calculate attraction and repulsion - can cause the aggregate as a whole to behave differently.

Although, as can be seen from this description, the AR framework allows for the creation of individuals with detailed and specific attraction and repulsion rules, models created in the AR framework are not required to, and frequently do not directly attribute to individuals in the model properties that are typically used when describing actual organisms- size, shape, colour, physical abilities, perceptual abilities, cognitive abilities, movement abilities. Instead, these properties are either ignored, incorporated abstractly into the attraction and repulsion equations or, in some of the more recent models mentioned in section 3.3 , incorporated into the model on top of the AR framework in an ad hoc manner. As a result, individuals in the AR framework are largely, if not entirely, defined by their position, their velocity and their attraction and repulsion equations. This minimalist quality of the AR framework has its advantages when it comes to understanding the underpinnings of aggregation. However, it can cause problems when the framework must be applied to specific aggregating species. In particular, as has already been 
stated, the lack of familiar, observable properties makes it difficult to successfully match, a priori, a particular theoretical model of aggregation with a particular aggregation in the wild.

Nonetheless, it is possible to use empirical data to do some matching of models to specific aggregates, even in the context of the AR framework. One possible approach for generating an IBSEM model of a particular animal aggregate using the AR framework (as suggested by this thesis) is as follows:

1. Observe the animal aggregate as a group. Make group-level observations about the aggregate (average size, density, speed).

2. Observe the behaviour of individual animals within the group. In particular, make observations regarding behaviour of individuals in relation to each other- their spacing with respect to other individuals, their orientation with respect to other individuals, and their movement patterns over time.

3. Develop hypotheses regarding appropriate attraction and repulsion rules for individuals within the aggregate, based on observations of the group and behaviour of individuals within the context of the group. Develop attraction and repulsion equations based on these observations.

4. If possible, observe small groups of animals interacting in order to confirm that the developed attraction and repulsion equations adequately describe the position of animals within the aggregate over time (e.g. Krause and Tegeder, 1994).

5. Create a model based on the observed behaviours of individuals within the aggregate.

6. Compare the group-level properties of the model with the group-level properties of the animal aggregate. If the group-level properties of the model do not reflect the group-level properties of the actual aggregate, gather more data and adjust the individual properties (e.g., relevant neighbours, attraction and repulsion equations, movement algorithms) until the group properties of the model match the group properties of the aggregate. 
The final result of this approach is a model with attraction and repulsion rules that have been inferred from the available empirical data. In a sense, the general concepts of attraction and repulsion have been custom fitted to the aggregate of interest. As such, the resulting model is, in some respects, a convincing model, since it displays observable and expected group-level behaviours for that particular aggregate, and these group-level behaviours are generated by the behaviour of the individuals within the model. Unfortunately, despite these apparently positive results, this model will run into difficulty when applied to certain problems of interest to animal aggregate researchers, as discussed below.

\subsubsection{Problems with Resulting Aggregate Models}

Despite the fact that a model constructed using the method described above is closely matched to the behaviour of the aggregate for the specific scenario being considered, there remains a notable disconnect between the properties of the modelled individuals and observable properties of actual organisms. Specifically, individuals in the model are still only defined by their position, velocity and attraction and repulsion equations. They are not specifically defined by biologically distinctive, observable properties - size, shape, colour, age, social position, health, perceptual abilities, cognitive abilities, movement abilities - that animal researchers tend to associate with particular species. This disconnect is not such a problem if the goal of the modeller is simply to create a model that succinctly describes how a collection of homogeneous individuals are theoretically able to form and maintain an aggregate. It is a problem, however, when creating species-specific models whose goals are to describe and predict the aggregate behaviour of a particular aggregating species under more variable circumstances. It is also a problem when generating novel predictions in order to validate such a model.

To more clearly appreciate the problems, consider using a model constructed in the manner described above to predict the behaviour of a particular aggregate under changing environmental circumstances. For instance, suppose that the level of light in the environment of the aggregate were to change over time. In this case, how should the attraction and repulsion equations be changed? Should individuals react by becoming more attracted to individuals, or less attracted to 
them? Or should, instead, both attraction to and repulsion from other animals become a weaker influence on the behaviour of the individual? In general, it is unclear how the AR equations should be altered as the environment is changed. As a result, it is hard to use these models to make predictions about aggregate behaviour under changing environmental circumstances.

The lack of connection between the AR framework and known animal properties also becomes a problem when trying to use this methodology to model a heterogeneous aggregation of animals. It seems likely that in the case of a heterogeneous aggregation, different animals in the aggregation might have different attraction and repulsion behaviours, as a result of variability in the underlying mechanisms being used to carry out these behaviours (due, for example, to age, size, social position, health, energy level). One way to capture this heterogeneity using the described methodology might be to first derive average attraction and repulsion equations for individuals within the model and then to vary these attraction and repulsion equations for different individuals within the aggregate model. Unfortunately, it is not clear how the aggregation equations should be varied with respect to individual differences between actual animals within the aggregate. For example, should larger animals be more strongly attracted to other animals, or less? How should the attraction and repulsion equations reflect this? And what experimental evidence should the modeller use when making these decisions? Again, these issues are not as much of a concern when investigating aggregate behaviour in a very abstract sense. They become a problem, however, when trying to understand the behaviour of actual aggregates (e.g., the behaviour of herds of female caribou (Rangifer tarandus) with their calves (see Miller, Jonkel and Tessier, 1977; Fancy, 1983; Harrington and Veitch, 1991)).

Validation also becomes a challenge for these models. Using the method described above, the behavioural rules for individuals are derived by researchers based on observations of the behaviour of individuals within the context of the aggregate as a whole. In other words, individual properties are derived from group properties. Because of this, the resulting individual aggregate rules are effectively guaranteed to produce the aggregate behaviour in question (Parrish et al., 2002), because they are simply the result of reverse engineering. This means that modellers must be careful about the ways in which they validate their species-specific models. They cannot, for example, argue that their models are valid based solely on the fact that 
individual behaviours successfully combine to produce the desired aggregate behaviour. This is simply an expected outcome of the modelling methodology. Instead, they must validate their model in some other way, perhaps by using it to make predictions about the aggregate behaviour in novel circumstances. Unfortunately, as discussed above, it is difficult to use models constructed in this manner to make novel predictions about the behaviour of the specific aggregate of interest in new environments or by altering the composition of the aggregate in some way.

These problems may be overcome by incorporating into the model at least some properties of individual behaviour that are derived from observations of individuals alone, and which are clearly tied to properties and behaviours of the species of interest. Aggregate behaviours that result from these individual behaviours will then also be connected to the species of interest. However, this raises the question of which aspects of the AR framework are directly observable. The main features of the AR framework- attractive and repulsive social forces- are not directly observable, because animals do not literally exert attractive and repulsive forces on each other. Other model elements (e.g., agent body shape, agent perception, agent movement) are also generally abstracted in order to increase the generalisability of the model. Consequently, tying AR models to specific aggregates is difficult at best.

\subsection{Moving Forward: A cognitive science solution}

\subsubsection{The problem of verifying models}

As might be anticipated in light of the problems discussed in section 3.1, some animal aggregate researchers (e.g., Parrish and Edelstein-Keshet, 1999; Rohani, Lewis, Grünbaum and Ruxton, 1997) are critical of the idea that IBSEM aggregate models can be informative with respect to the behaviour of particular aggregating species or groups of species. Their scepticism is largely derived from the fact that a wide variety of behavioural rules can produce IBSEM models that exhibit aggregate behaviour. Putting this concern succinctly, Parrish and Edelstein-Keshet (1999, p.101), state " $[\mathrm{M}]$ any sets of rules can lead to lifelike group behaviour, so that the results, though visually appealing, may be uninformativ". In other words, aggregation is a multiply realisable phenomenon. As a result, it is difficult to determine when an aggregate model truly 
reflects the behaviour of a specific aggregating species or when it does not but simply looks like it does. It is the hypothesis of this thesis that in order for IBSEMs to be fully accepted as an animal aggregate modelling tool, and for aggregate researchers to gain the explanatory power offered by IBSEMs, researchers must demonstrate that the models they produce are sufficiently constrained such that they are a match to the actual aggregate of interest rather than to animal aggregation in a more general sense.

To achieve this, researchers need to produce experimentally verifiable models of animal aggregates. Generally speaking, in order for a model, IBSEM or otherwise, to be validated through experimental evidence, the component parts of the model must first be clearly and explicitly connected to the phenomenon of interest. This connection constrains the design of the model so that the behaviour of the model cannot simply be adjusted in an ad hoc manner until it matches the behaviour of the phenomenon. As a result, the behaviour of the model can then be compared with experimental evidence in order to validate the model. In the case of IBSEMs, which are focused on and driven by individual behaviour, this means that the model must be connected in some way to the phenomenon of interest at the level of the individual. If relevant properties of individuals in the model are clearly matched with relevant properties unique to the animal of interest, then the model will be constrained in such a way that it is provably a model of the specific phenomenon of interest.

Some researchers have already recognised that, to improve the current modelling situation with respect to models of specific animal aggregates that are supported by experimental data, researchers need a more concrete and behaviourally detailed animal aggregate IBSEM modelling framework. Parrish et al. (2002) note, in the context of models of fish schooling, that the current inability of modellers to directly compare models and resolve conflicting model results might be remedied by the presence of a common fish school modelling framework. This point made by Parrish et al. in the context of fish schooling- that a common modelling framework is required to move research forward - is also applicable to animal aggregate modelling more generally. Ideally, this common framework would find ways to overcome the current difficulties that modellers in this area face when trying to connect their models to the behaviour of a 
particular aggregating species, in addition to allowing them to compare their resulting models with other aggregate models.

One of the requirements for developing a general aggregate modelling framework is that the modelling framework in question must be specific enough to produce models that can be validated against actual observations of biological phenomena, but general enough to be used for many different types of animal aggregates. As well, ideally, any proposed framework would build on existing research by being compatible with, but less abstract than, the well-established AR framework. Cognitive science provides a possible solution to these challenges.

A key principle in cognitive science research is the view of living organisms as processors of information (Holyoak, 1999). Although there are competing information processing modelsfor example Gibson's ecological psychology approach (1977) vs. the more traditional representational approach (Neisser, 1999) - it is generally accepted that certain types of energy (sound waves, light waves, etc.) cause animals' sensory organs to react. These reactions then propagate a chain of further reactions, which can be interpreted as the processing of information: perceptual information processing abilities are associated with each sensory organ, cognitive mechanisms receive information through these perceptual processes and the behaviour of the animal is altered as a result of this cognitive information processing (see Figure 7). The entire sequence can be characterised as the reception and use of information by the organism.

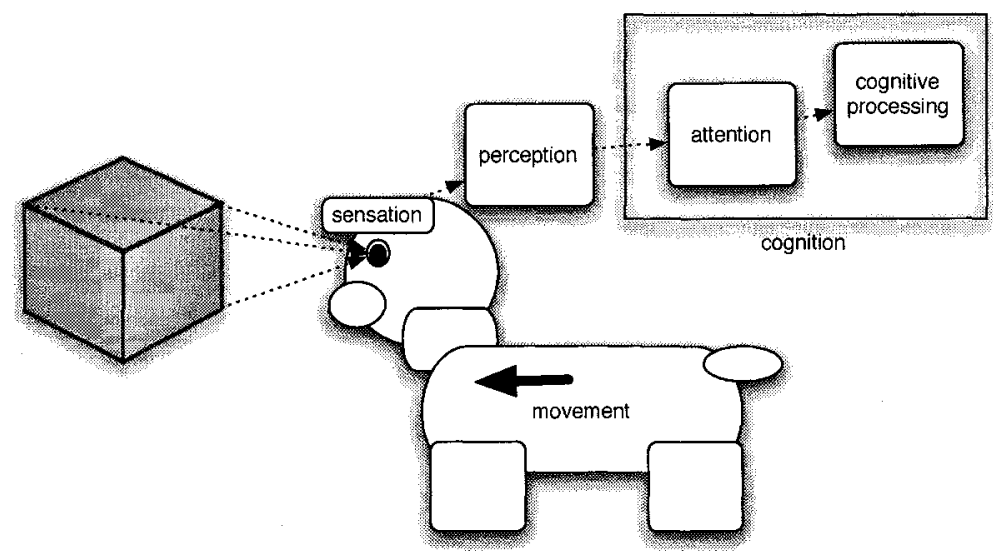

Figure 7: Information processing: Information, represented by the dotted line, is received through the senses, preliminarily processed by perceptions, filtered by attention and then cognitively processed. Movement is one result of information processing. 
The information processing paradigm is a strength of cognitive science, because it allows cognitive scientists to bridge multiple disciplines and compare research across multiple species. In the case of animal aggregate research, it can also provide a means for the creation of a multi-agent, biology based framework of aggregation that is general enough to be applicable to many different aggregating species, but concrete enough to allow for the validation of specific animal aggregate models. It can do this because the information processing paradigm works across species but, at the same time, distinguishes between species by distinguishing between the type and amount of information that can be processed by each species.

\subsubsection{The implicit role of information processing in the AR framework}

Even the most abstract implementations of the AR framework do have one connection to the cognitive science concepts of information processing, as well as to more concrete, biological mechanisms of aggregation. Specifically, aggregate modellers, mathematical and simulation based alike, almost always assume that there is some limit to the area or volume of space within which an organism is influenced by other members of the aggregate. More specifically, the members of an aggregate that influence the behaviour of a particular individual within the aggregate are frequently defined as those members of the aggregate that are within a certain radius of the individual in question. Attraction and repulsion equations for the individual then operate only on this limited subset of aggregate members rather than on the total number of individuals present in the environment.

This restriction of influence in the model is linked, by some modellers in a general way while by other modellers very specifically, to the idea that, since real organisms use their senses to receive information about the other members of the aggregate, and the perceptual abilities associated with these senses to do preliminary processing of this information, it is more biologically plausible in models to restrict the amount of information an organism can receive than to allow them access to all available information in the environment. Grünbaum and Okubo (1994), for example, describe Lagrangian models (i.e., individual based mathematical models) as typically incorporating what they refer to as a 'sensing range', which limits the interactions 
between individuals (see, for example, Mogilner et al. 2003; Tu 2000). Similarly, as discussed, Zheng, Kashimori, Hoshino, Fujita and Kambara (2005), in their IBSEM model of the reaction of fish schools to attack by a predator, provide agents with what they term a 'visible area', outside of which the agent does not receive information about the environment. This is in keeping with the idea that actual senses and perceptual abilities are limited and imperfect (for further examples of this, see Aoki, 1982; Couzin et al, 2002).

As was discussed in section 2.4.2, other researchers have made more explicit efforts to included sensation and perception in their models, in some cases modelling perception in a general sense (as in Viscido et al. (2002)) while in other cases specifically referring to the sensory and perceptual abilities of a particular species (as in Ward, Gobet and Kendall (2001)). Most explicitly, Hemelrjik and Hildenbrandt (2003, 2008), in their models of fish schooling, specifically refer to their agents as having a 'range of perception', and adjust the shape of what they refer to as the agents 'behavioural zones' based on data about lateral line ranges of fish species. Viscido et al. (2005) also restrict the angle of the volume in which agents react to other agents to \pm 150 degrees, based on the fact that most fish have a rear blind spot. Thus, while in some models the role of perceptions and the types of information received by these perceptions are mentioned only in passing or are left unacknowledged altogether (e.g., Adioui, Arino et al. 2003; Toner and Tu, 1998), in other models perception is clearly alluded to as being relevant.

These latter models are still lacking in various ways, however. Generally speaking, the perceptual acuity, the area or volume over which the perceptions operate and the nature of the information being received by these perceptions is not based on experimental evidence specific to the species being studied (if based on experimental findings at all). As well, even when a reference is made to perceptions in the model, researchers fail to make a clear distinction between the perceptual, attention and cognitive abilities of the species being modelled. Instead information restrictions are generally viewed as being associated with the attraction and repulsion equations and are not separated into perceptual, attentional and cognitive processing components. 


\subsubsection{Updating the AR framework using the information processing paradigm}

By acknowledging that agents do not have perfect knowledge of their surroundings, and restricting access to environmental information using an area or volume in which agents can receive information, aggregate modellers are implicitly recognising the key role of perception mediated information processing in aggregation. There are a number of theoretical and experimental advantages to be had in making this implicit role explicit, and clearly connecting the physical space in which model agents are able to receive information to the perceptual abilities and aggregating behaviours of the animal being modelled (see Figure 8). First, and most importantly, perceptual, cognitive and movement abilities can be determined through the experimental study of individual animals, rather than groups of animals, because these are independent properties of the individual rather than group dependent properties. Alignment, for example, is a group dependent property because it exists only in the context of two or more individuals). This, in turn, allows for emergent group properties, rather than assumed group properties, and overcomes some of the modelling difficulties discussed in section 3.1.2.

Secondly, animal behaviourists already have some understanding of how perceptions, cognition and movement are affected by the state of the environment and other properties of the individual (for example, how vision is affected by light levels (Levenson and Schusterman, 1999) or how visual acuity changes as an organism ages (Schneck, Haegerstrom-Portnoy, Lott, Brabyn and Gildengorin, 2004)). These effects may then be incorporated into models of aggregation and used to make predictions about the behaviour of actual animal aggregates in particular environmental conditions.

Finally, since a perception-focused information processing framework is consistent with the AR framework, theoretical work may still be carried out using the AR framework. However, in instances where specific animals are involved, an information processing framework that takes into account the perceptual, cognitive and movement abilities of the animal being modelled can be used and data from animal behaviour research more directly incorporated into aggregate models. The connection between aggregate behaviour and observable animal properties will 


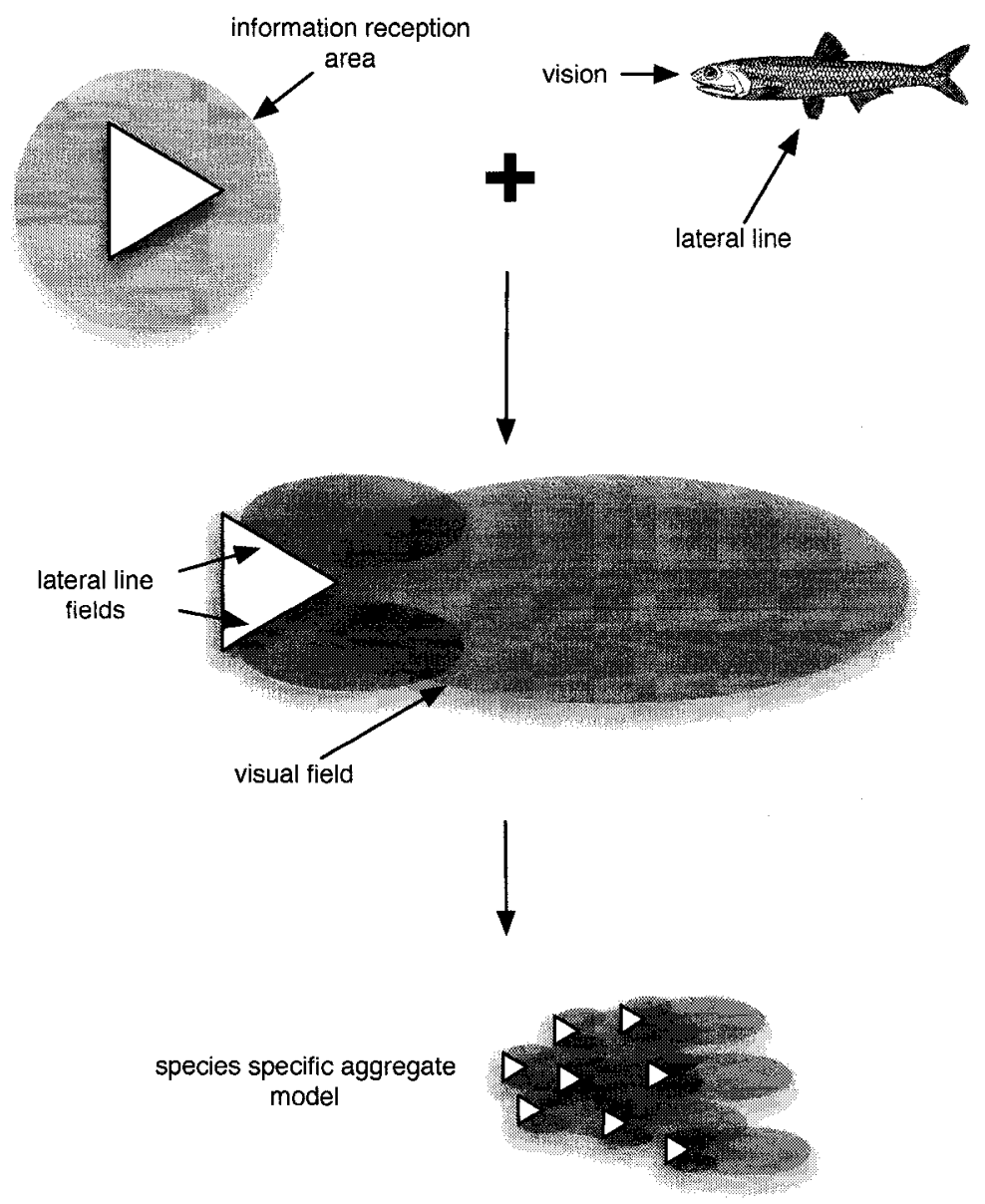

Figure 8: Combining the concept of the information area with what is known about animal perception results in an experimentally validatable model of individual agents. This in turn allows modellers to create species-specific aggregate models . Perceptual field details are discussed in Section 3.3.2.(Anchovy drawing in figure from (Pearson Scott Foresman, n.d.) 
also serve to connect animal aggregate models more directly to experimental research on animal aggregation, as well as to the larger field of animal behaviour.

\subsection{The PCA framework}

This section presents the PCA framework. This framework is intended for use by researchers, in conjunction with the IBSEM modelling methodology, for the purpose of developing experimentally verifiable models of species-specific animal aggregates. The PCA framework will allow researchers to do this by drawing a connection between observable and measurable properties of actual aggregating animals - including perceptual, cognitive and movement abilities - and the properties of agents in the IBSEM models.

\subsubsection{Framework Requirements}

For the PCA framework to be an improvement on the existing AR framework several requirements must be met. First, the new framework must retain the good qualities of the AR framework. Specifically, it must be abstract enough to apply to many different groups of animals. It should also be the case that a relatively small number of parameters are required by the framework, so that it can be used to create models in a simple and straightforward manner. Finally, all modelling concepts must be clearly and concretely defined so that guesswork is not required when it comes to implementing the concepts in a particular model.

Second, the new framework should allow biologists to use their existing knowledge of the perceptual, cognitive and movement properties of organisms in their construction of models. For example, if biologists already have information about the perceptual or movement abilities of the European swallow (Hirundo rustica), aggregate researchers should be able to easily incorporate this knowledge into a model of European swallow aggregation, using the PCA framework. In particular, biologists should not have to translate significantly between the concepts they are already using to gather this type of information about organisms and the proposed modelling concepts. 
The resulting models must also tell biologists something about the relationship between individual behaviours and properties and group behaviours and properties and be empirically verifiable as models of the particular organism in question. This means that in addition to being well defined in terms of implementation, the parts of the model must be well defined with respect to the organism being modelled. Lastly, the properties and behaviours of the organism that are related to the parameters and components of the model should be ones that are generally believed, by biologists, to contribute to aggregate behaviour.

\subsubsection{Details of the Modelling Framework}

The PCA framework (see Figure 9 and also Chapter 4, Algorithm 1) is a general framework for modelling species-specific animal aggregate behaviour. Agents in PCA based models have clearly defined perceptual abilities and cognitive algorithms, with a marked distinction between the perceptual processing stage of the agent and the cognitive processing stage. Perceptual and movement abilities of agents can be set based on available experimental data for the specific animal being modelled. Cognitive algorithms and parameters can be based on experimental data or constructed with consideration given to plausible cognitive mechanisms of the animal being modelled. The PCA concepts developed to characterise all of these properties will be described in this section.

Perceptual field Motivated by the requirements discussed in section 3.3.1, the two PCA concepts that relate the perceptual properties of individual animals to aggregate behaviours are the perceptual field and perceptual resolution. Consider first the concept of the perceptual field (see Figure 10). For the purpose of modelling aggregates, the perceptual field can be defined as the space within which a particular perception (e.g., visual perception) of a particular organism (e.g., a hawk) can receive processable information about relevant aspects of its environment. For example, it is common to say that a hawk can see a rabbit up to a mile away, which, in the PCA framework, is analogous to saying that the visual perceptual field of the hawk (with respect to rabbits) extends outwards one mile. 


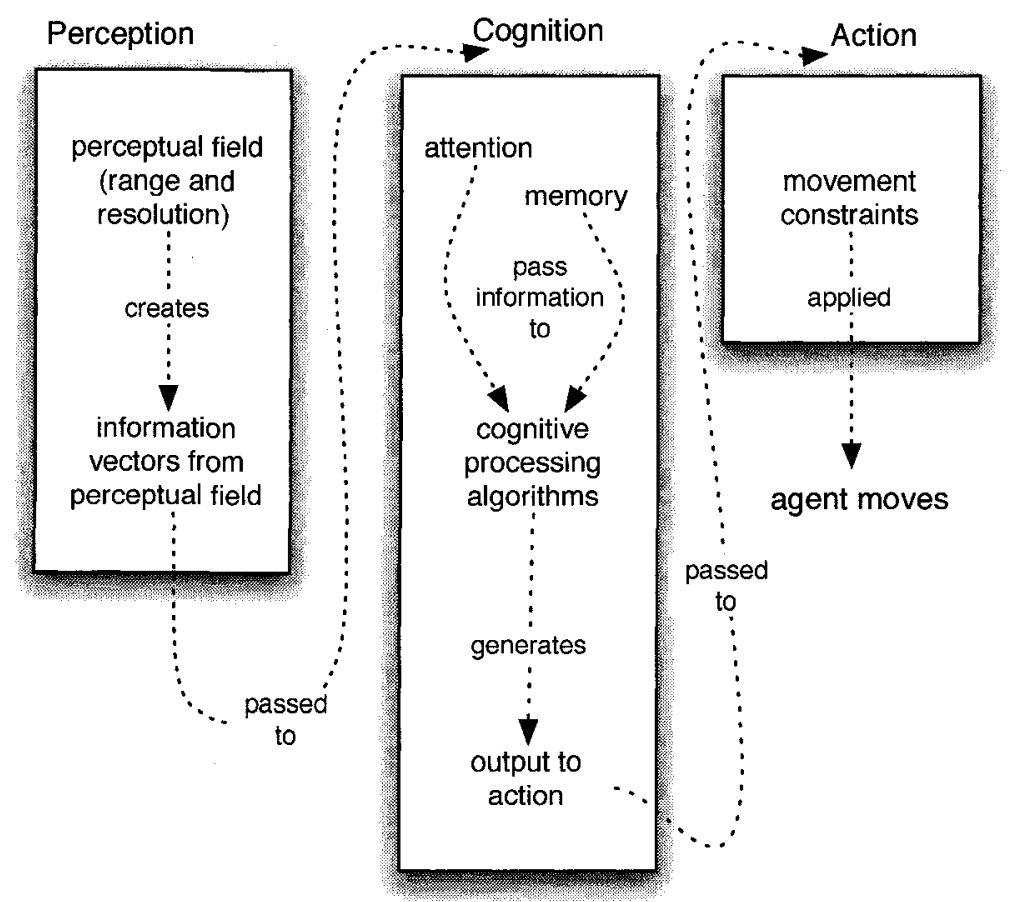

Figure 9: A schematic of the PCA framework. See also Chapter 4, Algorithm 1.

This is a simplification of perceptual functioning, since it is more accurate to say that sensory organs react to those part of the environment that come directly into contact with them (e.g., light waves interact with the surface of the eye). These reactions are then transformed by the perceptions into usable information about the environment, as constrained by the level of acuity of the sensory organ in question and the processing ability connected to that sensory organ. Both sensation and perception are modelled in the PCA framework by having perceptual fields provide a constrained amount of information to agents over a certain area in space. Generally speaking, there is expected to be at least one perceptual field associated with each sensory ability that is considered relevant to the behaviour of interest. The specific number of perceptual fields and their shape will depend on the level of detail required by the model.

Formalising the concept of the perceptual field for a particular model requires defining a space over which a particular perceptual ability functions. For example, the visual perceptual ability of a particular type of bird might be represented as two perceptual fields, represented by two three dimensional cones with length $1000 \mathrm{~m}$ and an angle of $180^{\circ}$ originating on either 


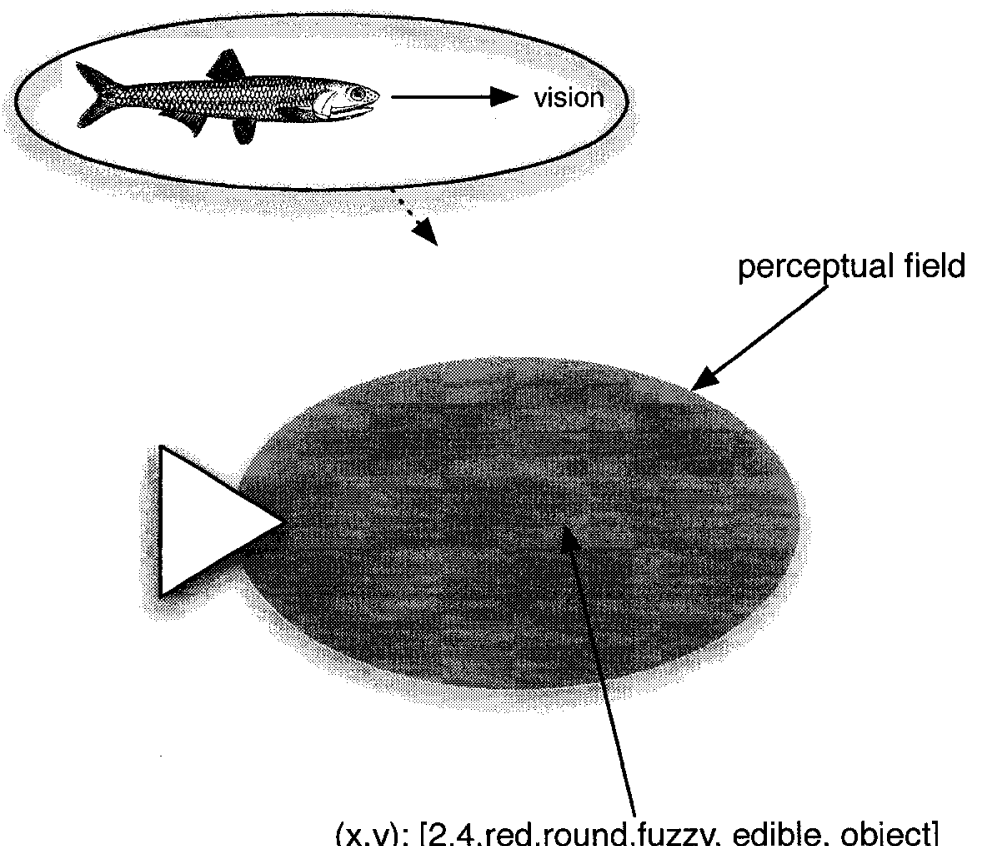

Figure 10: An agent's perceptual field. This field, derived from experimental data, determines the area or volume over which an agent can receive information relevant to a particular perception. Each point in the perceptual field can be associated with a certain amount of potentially available information.

side of the bird's head. The precise nature of the representation will depend both upon the level of detail desired in the model and the nature of the experimental data available. The bird's two visual perceptual fields represent the region of space within which the bird can receive useful visual information. Information within this region may still remain undetected or be misinterpreted, but information outside this region is not available to the organism at all through the particular perception being considered.

A perceptual field $(F)$ can be defined mathematically as a vector field consisting of the set of all points within a particular space (in two or three dimensions, depending on the model), where this space is defined and constrained using mathematical equations. For example, a perceptual field may be defined using a cone equation, with the set of perceptual field vectors $(P)$ constrained to be all points, $\vec{p}$, within a cone, where $\vec{C}$ is the vertex of the perceptual field cone, $\hat{d}$ is a unit length direction vector, $r$ is the radius of the perceptual field cone and $\theta$ is the angle of the cone. (see equation 5). 


$$
P=\text { all points, } \vec{p} \text {, such that } \hat{d} \cdot(\vec{p}-\vec{C}) \leq|\vec{p}-\vec{C}| \cos (\theta) \text { and }|p-C| \leq r
$$

The advantage of the perceptual field concept is that it is immediately applicable to organisms ranging from slime mould cells (which a researcher might model as having a perceptual field the shape of a two dimensional ring around the outside of the cell, within which the cell can react to the presence of other cells) to humans (which a researcher would need to model, depending on the scope of the model, as having multiple perceptual fields, each with a particular shape corresponding to sight, smell, hearing, etc.). Most importantly, the perceptual field for a particular organism can be specifically defined for that organism using experimental data, thus connecting the model clearly to that organism.

Perceptual resolution The next parameter, closely associated with the perceptual field, is perceptual resolution. The perceptual resolution of a particular perceptual field can be generally represented by a perceptual resolution tuple, $\tau$. Each item in the tuple represents a detected object or environment property, described by a name (e.g., <xcoordinate, colour $>$ ). Connected with each name is a list of possible values for that property (e.g., values for the colour property might be white, red or black). The size of the tuple is the same as the number of properties for which the perceptual field in question can obtain information. The amount and type of the information available to the individual within the context of this field will depend on how the particular perception operates and thus on how the perceptual resolution associated with the field is defined.

At a given time, the set of perceptual resolution tuples, $R$, represent the information actually returned to the individual by a particular perceptual field, $F$ (see Figure 11). To be more precise, the perceptual resolution tuples of the perceptual field can be described as a transformation, $T$, of the set of perceptual field vectors, $P$, associated with the perceptual field:

$$
R=T(P)
$$

where $P$ is the set of perceptual field vectors and $R$ is the set of resulting resolution tuples. For particular models and perceptions, the modeller can establish a specific function, $T_{\text {model,perception }}$ 
that connects $P$ to $R$ in an appropriate manner (i.e., that takes into account the information plausably available to the perception, sources of sensory error and level of representation appropriate for the particular model).

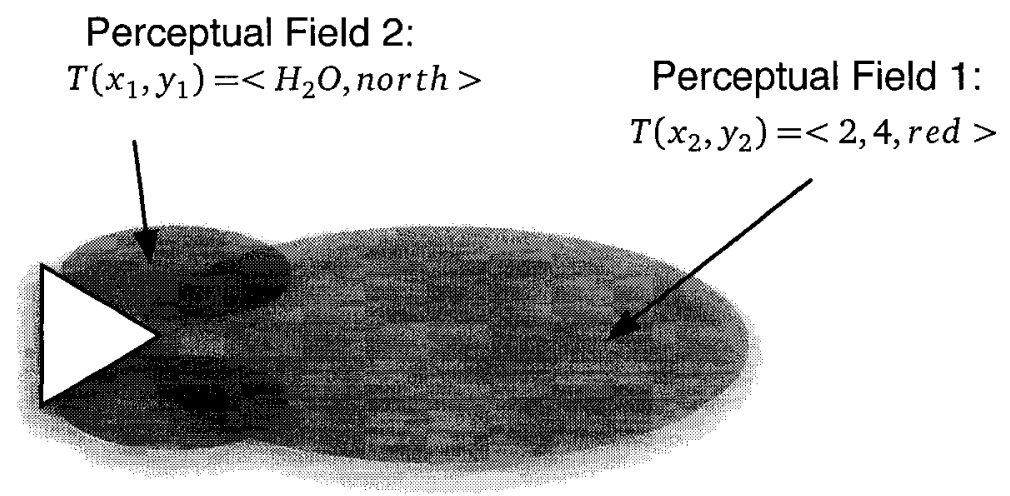

Figure 11: The perceptual resolution of an agent: Each different perceptual field can return different types and amounts of information to the agent. Each point in a perceptual field will return different values, which depend on the information available in the environment.

The resulting tuples (perceptual resolution tuples, $\tau$ ) contain the actual information available to the animal in question. For example, consider a perception that can determine the chemical composition and approximate direction from which a particular chemical was originating. The perceptual resolution tuple could be generally described as < chemical, direction $>$ and a specific perceptual resolution tuple might be $\left\langle\mathrm{H}_{2} \mathrm{O}\right.$, north $\rangle$.

The information contained within the perceptual resolution tuples at a specific instance in time depends on the type and amount of information the perception in question can receive, the particular part of the environment that is currently covered by the perceptual field associated with that perception and any error functions that the modeller wishes to incorporate into the field. Thus, the perceptual resolution of the visual perception of a particular type of bird might allow the return of information (with some degree of error) about the spatial location and colour of certain types of objects that are within its visual perceptual range, which, as in the example above, might be two three dimensional cones with length $1000 \mathrm{~m}$ and an angle of $180^{\circ}$ originating on either side of the bird's head.

Perceptual resolution is particularly important because it is a distinguishing factor among different species of organisms and, within species of organisms, different subgroups of organisms 
(different ages, sexes, etc.). This means that its inclusion in animal aggregate models will allow the models themselves to distinguish between species and subgroups. For example, it is possible that the echo-location perception of bats and the visual perception of birds might be reasonably represented by the same perceptual field shape and size. However, the nature of the information obtained within this field - the perceptual resolution of the field - might be quite different, thus distinguishing between models of these two species. For example, both birds and bats might receive information about object location, but only the bird would receive information about the colour of the objects. In this respect, perceptual resolution can also usefully serve to highlight or compare similarities and differences between species.

Cognition: Attention and cognitive processing The perceptual field and perceptual resolution act to constrain which theoretical models can be associated with a particular aggregate group. They also provide an experimental connection between the model and the actual aggregate of interest. However, they are not the only constraints available to the modeller. In addition, cognitive abilities and movement abilities of the animal being modelled may also impose constraints on the behaviour of the individuals within the model.

Formally, cognition in the PCA framework (see Figure 12) may be described as a series of equations (or algorithms) that select and perform calculations on the environmental information obtained through the perceptions. These equations may also involve internal (memory) parameters that are either constant or set based on previous calculations. The result of these calculations is a movement decision, which is further constrained by the movement abilities of the animal in question (discussed in section 3.6.5).

In the PCA framework the broader component of cognition is broken down into two distinct subcomponents- attention and cognitive processing. The concept of attention refers to those cognitive algorithms or functions, $A\left(R_{1}, R_{2}, \cdots, R_{n}\right)$, that select which parts of the information received through the perceptual field will be passed to additional cognitive algorithms and which will be ignored. The term cognitive processing refers to algorithms or functions, $C(A, M)$ that perform calculations using the selected information, in conjunction with available internal information, $M$ (e.g., memory, internal states) to determine outputs, $I$, to the movement part of 


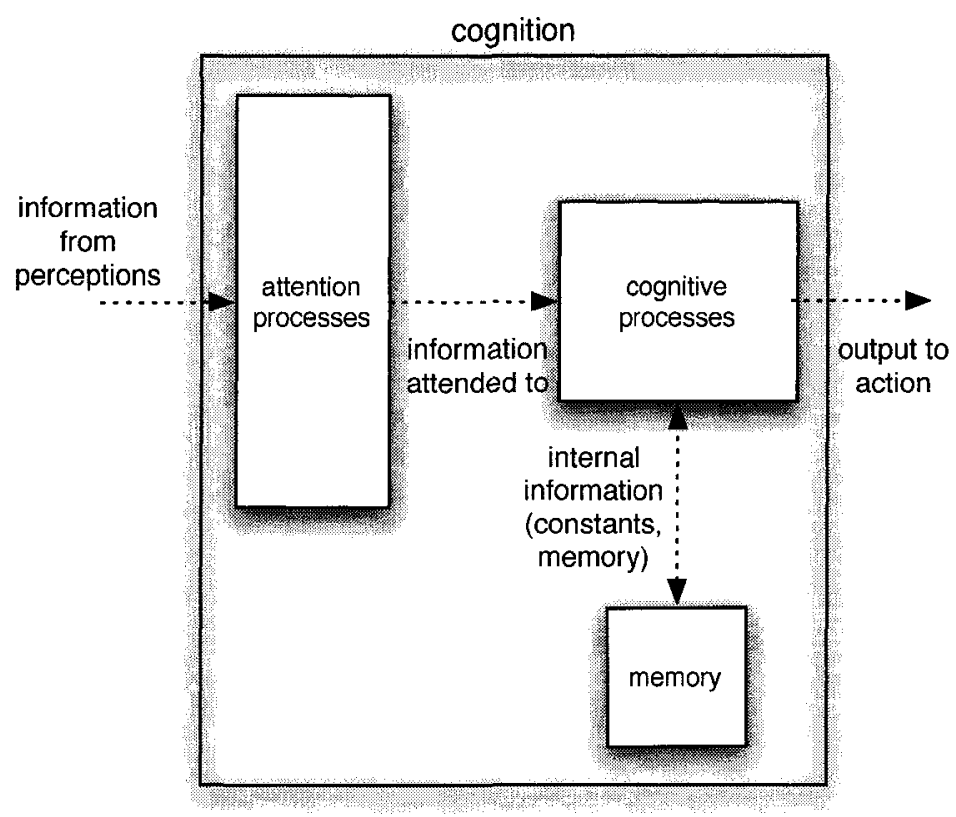

Figure 12: An overview of the cognitive structure.

the model (see Equation 7). Information may be unavailable to an agent because it is not within that agent's perceptual field. However, information may also be available to the agent from a perceptual perspective but then be ignored or become unavailable during attention or cognitive processing.

$$
I=C\left(A\left(R_{1}, R_{2}, \cdots, R_{n}\right), M\right)
$$

Thus, the PCA framework requires modellers to specify which parts of the model pertain to perception, which parts of the model pertain to attention (determining which information will be used by cognitive processing) and which parts of the model pertain to the actual cognitive faculties of the animal being modelled. The modeller must also be specific about the transfer of information between these stages, decide at which stage information becomes unavailable, is lost or discarded, and show how cognitive processes lead to movement decisions.

This explicit representation of cognition means that modellers must transition from describing movement behaviours towards and away from other aggregate members as metaphorical 
forces (a representation which modellers already acknowledge to be a convenient fiction (Helbing and Molnár, 1995)) to portraying them as outcomes of cognitive processes. At the modelling level, rather than having agent position in a model automatically adjusted as a result of force equations acting on the agent, in PCA models the path of information leading to the generation and selection of attraction and and repulsion behaviours is modelled directly. Effectively, the agent gains agency, rather than being modelled as a simple object upon which forces act. This in turn means that the cognitive load being placed on the animal being modelled and any cognitive challenges that must be solved by the animal (e.g., the binding problem (Dennett, 1987), the frame problem (Singer, 1996)) also become explicit. As a result, mechanisms that are presented must be at least theoretically available to the animal in question (see Chapter 4, Sections 4.1.4 and 4.3.4 for additional discussion of this).

Movement Movement is an important component of the PCA framework because it completes the loop from perception to cognition to action and back to perception again. Movement choice (action, $\Omega$ ) is a function of outputs from cognition $(I)$, with constraints imposed by the physical abilities of the animal $(\Upsilon)$ and the environment $(\Xi)$. As an animal in an aggregate moves, the volume encompassed by its perceptual field moves. Movement also results in animals moving into the perceptual fields of other animals, even if these other animals are, themselves, stationary. Thus, the information available to perception is also a function of movement. This completes the cycle from perception to cognition to action and back to perception, with each part of the cycle determining the behaviour of the following step (see Equation 8).

$$
\Omega(I, \Upsilon, \Xi) \rightarrow P
$$

Consequently, accurately modelling the movement abilities and restrictions of the species of interest is an important component of the PCA framework.

Although movement is arguably the most easily quantifiable and observable individual property of the three components of the framework, and can thus provide an important experimental basis for the aggregate model, it remains a challenge for IBSEM researchers to incorporate realistic movement rules and constraints into their IBSEM models. Partly this is because it is not 
always feasible to calibrate IBSEMs in such a way that there is a known relationship between the way space and time are represented in the model and the way space and time work in the real world. Partly this is because realistically modelling movement abilities and restrictions may require the use of physically realistic, three-dimensional modelling environments. Nonetheless, making a match between animal and agent movement, and then providing restrictions on the movement abilities of the modelled individuals based on this match, is worthwhile because it greatly increases the connection between the model and the aggregate in question.

\subsection{Problems Solved By the PCA Framework}

As discussed in the first part of Chapter 3, modellers using the AR framework to make speciesspecific aggregate models may run into difficulty when undertaking model validation and generating novel behaviour predictions. Modellers may also find it challenging to explore the ways in which a changing environment and agent heterogeneity influence aggregate behaviour. As well, due to a diversity of modelling approaches, researchers may find it difficult to compare and contrast their models, and the results of these models, with other aggregate research. Adopting the PCA framework will allow researchers to successfully deal with these problems, as detailed below.

Validating the model As discussed in Section 3.1, current aggregate models are difficult to validate because methods used to create the model effectively guarantee that the model will display the observed aggregate-level behaviour. To validate such a model, the model would need to predict novel behaviours, but, as has been discussed, current models have a difficult time predicting novel aggregate behaviour, with respect to a specific aggregating species, because it is not clear how changing the model relates to changing the environment of the actual aggregate.

The PCA framework avoids this problem because individuals in the aggregate model are more directly and experimentally connected to individuals within the actual aggregate. As

a result, group-level behaviour of the model is generated by observing individual behaviour, rather than observing group-level behaviour. This means that the model can make testable 
predictions about the group-level behaviour of the aggregate, which, in turn, allows the model to be validated against experimental evidence. Thus, the PCA's increased connection to the individuals within the aggregate allow species-specific models to be experimentally validated. Chapter 4 will demonstrate how the PCA framework can be used to create a validated model of D. rerio aggregation.

Changing the environment A changing environment will affect the nature of the information that each perception of the animal in question can receive. Models created using the PCA framework can readily incorporate these changes into the model. Specifically a change in the environment can be reflected by changes to both the shape of the perceptual field and the perceptual resolution of the field, for a particular perception. For example, in dim light the size of the visual perceptual field of an aggregating fish might be reduced and colour information might no longer be received, thus changing the perceptual resolution. It might also be relevant to add some amount of error to the information being received. Most importantly, these changes can then be verified experimentally. Meanwhile, other perceptual fields, like the lateral line perception in fish (which detects changes in water pressure around the fish), would be unaffected in this circumstance, since their functioning is not affected by light levels. By modelling each perception separately, the effects of environmental changes can also be modelled.

Heterogeneous aggregates Variability among aggregating individuals may also be more easily captured using the PCA framework. This is because differences between aggregating individuals that affect their aggregating behaviour will be reflected in differences either in their perceptual abilities, their cognitive abilities or their movement abilities. The PCA framework allows modellers to change the properties of agents in a manner that reflects these differences rather than simply changing the attraction and repulsion behaviours of individuals in an ad hoc, or averaging way.

For example, if a group of aggregating animals contains animals that vary widely in age, we might also expect to see differences in perceptual and movement abilities. Using the PCA framework, these differences can be modelled directly, at the level of the individual animal, 
again in a way that can be verified experimentally. The resulting behaviour of the aggregate will then clearly be as a result of these experimentally verified individual parameters.

Comparing and contrasting models As Viscido et al. (2007) have indicated, most aggregate model parameters have a relatively strong influence on aggregate behaviour. Consequently, if models differ across multiple algorithms and/or use different parameter values to examine effects, it is hard to compare these models with respect to changes across values of a single parameter, even if that particular parameter (e.g., attraction range) is shared by the models. One of the goals of the PCA framework is to improve this situation by providing modellers, first, with a way to consistently describe and compare similarities and differences between their models, and second, with a way to establish sets of common models that can be used to create results that are consistent and comparable with each other and also clearly comparable and relatable to biological aggregates. The PCA framework can also be used to characterise existing models by reframing and then re-describing these models in terms of perceptual fields, attention algorithms, cognitive algorithms (calculations resulting in movement decisions) and movement algorithms. A description of the properties of agents (e.g., shape, size, velocity, orientation, position) in the model and the dimension (e.g., one-dimensional, two-dimensional, three-dimensional) of the model is also useful. This characterisation allows for a more detailed and accurate comparison between models because similarities and differences become easier to detect. It also becomes easier to see what parameters are being varied for each model and the values being used in parameter sets.

\subsection{Chapter summary}

This chapter began by discussing several problems associated with using the AR framework to model species-specific animal aggregates. The chapter then presented, as a solution to these problems, the PCA framework, which centres around two perceptual concepts (perceptual field and perceptual resolution) two cognitive concepts (attention and cognitive processing) and realistic animal movement based on the results of cognitive processing. A general explanation 
of the ways in which these concepts can be used to create models of specific animal aggregates has been provided, along with a discussion of how the PCA framework resolves some of the modelling issues presented in the first part of Chapter 3. The next chapter, Chapter 4, will provide more detailed information on how the PCA framework can be applied in an experimental setting, and will provide a demonstration of the PCA framework by presenting the results of an IBSEM model of $D$. rerio aggregation created using the PCA framework. 


\section{Chapter 4}

\section{Testing the Perception Cognition Action framework: A $D$. rerio model}

In order to confirm the utility and viability of the PCA framework, the PCA framework was fully implemented in Breve, a three-dimensional, physically realistic modelling environment (Klein 2002). This PCA implementation (the PCAI) provides aggregate researchers with a concrete way to create models using the PCA, and was used in this thesis to create a species-specific $D$. rerio aggregation model. The goal of the $D$. rerio aggregate model was to generate group-level aggregate property predictions about the behaviour of an aggregate of $18 \mathrm{D}$. rerio by gathering data about the behaviour of individuals from this group, incorporating this individual data into the computer model and then analysing this model to obtain aggregate-level predictions. Successful predictions would provide support for the validity of the PCA framework as an aggregate modelling framework, as well as provide a case study for how the PCA framework might be used in a specific aggregate context.

This chapter will begin by describing how the PCA framework was implemented in Breve. The chapter will then describe the construction and analysis of the $D$. rerio model. Having generated predictions about the aggregate behaviour of the $D$. rerio aggregate being studied, in the last part of the chapter these model predictions will be compared with the behaviour of the actual aggregate, demonstrating that the model successfully predicts a number of aspects of the behaviour of the actual aggregate. As well, a comparison with the behaviour and predictions of a simple AR aggregate model will be made, demonstrating that, unlike the PCA model, its 
behaviour poorly predicts the behaviour of the aggregate, even when $D$. rerio movement and behavioural data are incorporated into the model.

\subsection{Implementing the PCA framework in Breve}

The PCA framework focuses on perception, cognition and action. In order to create speciesspecific aggregate computer models, each of these aspects of the framework, along with the interactions of these components, must be realistically implemented within a simulation environment. Because the PCA framework is a general theoretical framework, which can be functionally realised in multiple ways, it can be implemented in any modelling environment with the appropriate computational capabilities. The methodology for validating models using the PCA framework was initially developed and tested in a two-dimensional Netlogo environment (see Schellinck and White (2005) for a discussion of this project). For the purpose of this thesis, the PCA framework was implemented in Breve, a three-dimensional simulation environment designed for the implementation and visualisation of physically realistic IBSEMs (Klein 2002).

\subsubsection{Physical object implementation}

Each model object in the Breve PCA implementation (the PCAI) is represented by a set $(S)$ of geometric, three-dimensional shapes (e.g., spheres, cylinders, cones). More specifically, in this embodiment of the PCAI, objects are represented either as sets of spheres or, in the case of agents, spheres and cones. Restrictions are not placed on the positions of the spheres and cones that make up an object but they are generally expected to be positioned in such a way that their surfaces meet each other, to simulate a physically cohesive body (see Figure 13).

Objects in the PCAI have their own local coordinate system, and component shapes are positioned using this local coordinate system. The local coordinate system is then translated into the world coordinate system in order to move the PCAI object through the model environment. PCAI objects do not need to be symmetrical and can be rotated through any of the three axes, with all component parts rotating accordingly. 


\subsubsection{Agents}

The most common PCAI object is the agent, representing a single animal. Agent bodies, like all PCAI objects, are composed of multiple spheres, which are sized and positioned relative to the agent's local coordinate system. Agents also have perceptual fields, which are represented by single cones, with the set of all perceptual field cones associated with the agent denoted by $\Pi$ (see Figure 13). Like the spheres, these cones are positioned, sized and oriented relative to the agent's local coordinate system. As with any PCAI object, perceptual fields and agent bodies do not need to be constructed symmetrically. Agents may rotate in space, and body spheres and perceptual field cones rotate accordingly.

Agent bodies, since they are physically situated, have a position, $\vec{\rho}$, orientation, $o$, and velocity (comprised of speed, $s$, and direction of movement) represented by a velocity vector, $\vec{v}$. In addition to these basic properties, the modeller may also assign other properties to the agents using model variables. For example, the agent may be assigned colour, sex, age or disposition variables. The value of these variables are assigned during construction of the model and updated, if desirable, during the run of the model.

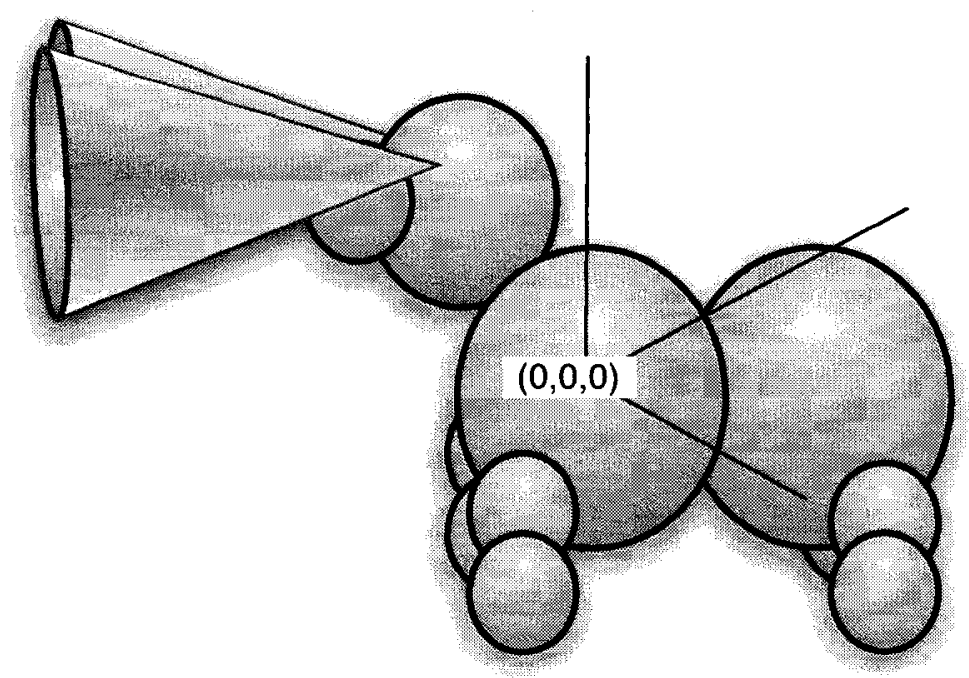

Figure 13: An example of a possible PCAI agent, with a body composed of spheres, two perceptual fields represented by cones and its own coordinate system. 


\subsubsection{Agent behaviour during one timestep}

PCAI models begin by setting up the model environment. The model then runs multiple iterations of the main model code. Each iteration of this code is called a timestep, and represents the passage of one unit of time in the model. Because variable values change during each iteration of the main model code, the result of running the code is different each time, and the model behaviour changes over time.

Generally speaking, at the beginning of each iteration, the model code asks each of the agents, $\alpha$, in the model to carry out a pre-defined series of actions, broken down into perception actions, cognition actions (attention and cognitive processing) and movement actions. Algorithm 1 provides a pseudocode outline of the PCAI's agent behaviour over the course of one model timestep (the $\operatorname{Timestep}(O, \lambda)$ function, where $O$ is the set of all objects $\left(S_{1}, S_{2}, \cdots, S_{\eta}\right)$ and $\lambda$ the set of all agents $\left(\alpha_{1}, \alpha_{2}, \cdots, \alpha_{n}\right)$. In Section 4.3.1, an algorithm-level description of the specific D. rerio aggregate model functions (Algorithm 2) will also be provided, demonstrating one possible instantiation of this pseudocode.

\subsubsection{Information available to agents: Agent perception and memory}

Agents have the ability to store information in memory using established memory variables $m_{1}, m_{2}, \cdots, m_{n}$, (see Figure 15). The values stored in these variables may be established when the model is being constructed, or may be calculated during the model run (the LEARN function in Algorithm 1). In addition, at each timestep, each agent receives information about other objects when the perceptual field cone of the agent intersects (the INTERSECT $(P, O)$ function, not shown) any sphere associated with the other object (note that occlusion is not modelled). Information about these objects, as mediated by the perceptual resolution of the perceptual field in question, is then returned to the agent as a list of tuples, $R$. In theory, it is possible for all available information about the object (all properties of the object) to be passed as a tuple to the detecting agent but this is unlikely to accurately reflect the perceptual abilities of the animal being modelled. Modellers constructing aggregate models using the PCAI must determine what perceptual resolution is appropriate for each perceptual field associated with an agent. 
$\operatorname{Timestep}(O, \lambda)$

1 for each agent in $\lambda$ :

2 do $L \leftarrow$ PERCEPTION $(O)$

$3 \quad L^{\prime} \leftarrow \operatorname{ATTENTION}(L)$

$4 \quad I \leftarrow$ COGNITIVE-PROCESSING $\left(L^{\prime}\right)$

$5 \quad \operatorname{ACTION}(I)$

PERCEPTION $(O)$

1 for $n \leftarrow 1$ to $|\Pi| \triangleright$ where $\Pi$ is the set of all perceptual fields, $\mathrm{P}$

2 do $L_{n} \leftarrow \operatorname{INTERSECT}(P, O) \quad \triangleright L_{n}$ holds information extracted from objects

3 return $L$

$\operatorname{ATTENTION}(L)$

$1 \quad L^{\prime} \leftarrow$ MERGE-AND-DISCARD $(L)$

2 return $L^{\prime}$

COGNITIVE-PROCESSING $\left(L^{\prime}\right)$

$1 \quad M \leftarrow$ RETRIEVE-MEMORY

$2\left(I, M^{\prime}\right) \leftarrow$ CALCULATE $\left(L^{\prime}, M\right)$

$3 \operatorname{LEARN}\left(M^{\prime}\right)$

4 return $I$

$\operatorname{ACTION}(I)$

1 CORRECT(I)

2 IMPLEMENT(I)

Algorithm 1: PCAI agent behaviour during one timestep. 
If a modeller chooses to create perceptual fields that overlap, it is possible for an agent to detect the same object through multiple perceptual fields (see Figure 14). How organisms combine information about the same object coming through different senses, and how they know which information to combine is part of a problem known in cognitive science as the binding problem (Hummel and Biederman, 1992; Singer 1996). Since it is likely that different animals solve the binding problem differently, a default algorithm for this is not supplied by the PCA or PCAI. Instead modellers who create overlapping fields must decide how they will resolve this for their particular aggregate model when writing their perceptual and attention algorithms.

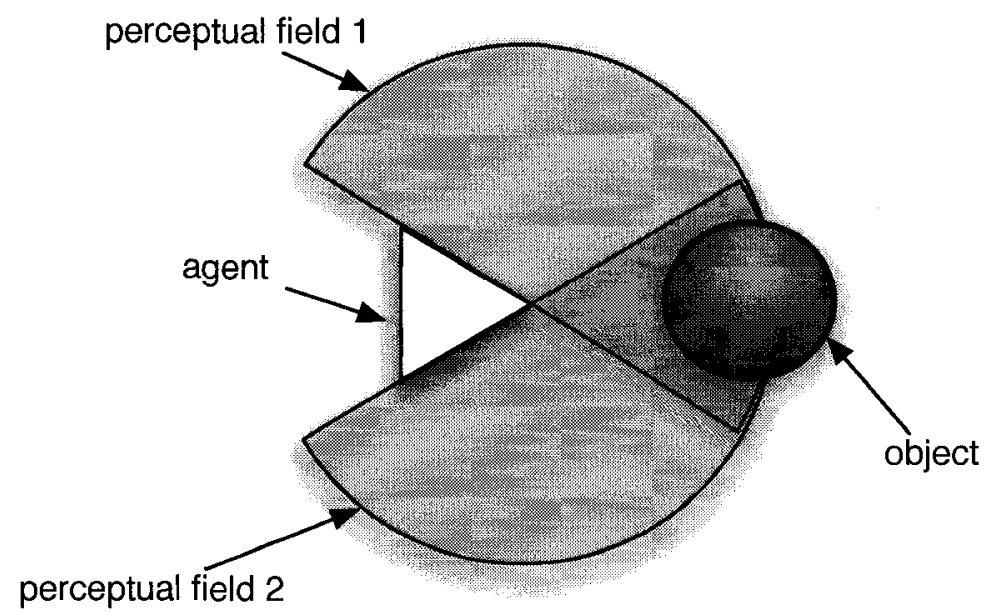

Figure 14: A PCAI agent with overlapping fields.

\subsubsection{Agent cognition}

Cognitive algorithms for agents are established during construction of the model. At each model timestep, the agent runs the cognitive algorithms, using as input the information from memory and the information returned by the perceptual fields (see Algorithm 1 and Figure 15). As described by the PCA framework, there are two cognitive stages- the attention stage and the cognitive processing stage. In the first stage, the attention stage, the PCAI agent receives a list, $\mathrm{L}$ of all tuple lists, $\left(R_{1}, R_{2}, \cdots, R_{n}\right)$ and determines which information to ignore, which 
information to combine and how this information will be passed through to the second stage of cognition (MERGE-AND-DISCARD in Algorithm 1). For example, the attention stage might randomly pick up to 4 of the object data structures returned by each perceptual field and pass this information on to the cognitive algorithm in a single list. In the second stage, the cognitive processing stage, cognitive algorithms perform calculations using available information, which includes information from memory and information received through the perceptual fields (CALCUlate in Algorithm 1).

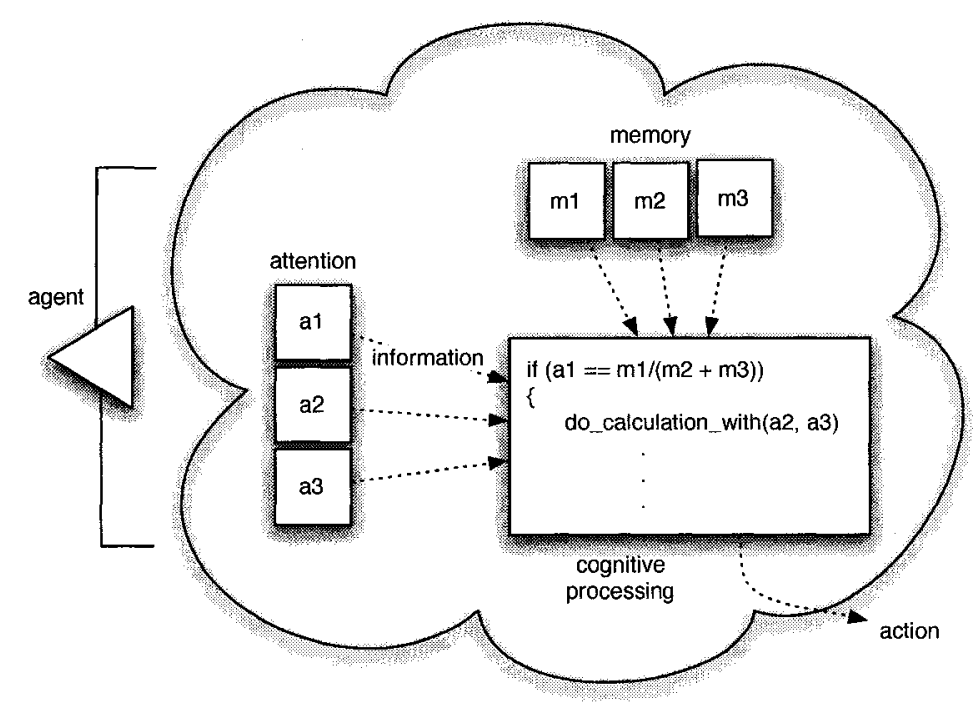

Figure 15: A PCAI agent with memory, attention and cognitive processing algorithms. Information from attention $\left(a_{1}, a_{2}, a_{3}\right.$ in the figure $)$ and memory $\left(m_{1}, m_{2}, m_{3}\right.$ in the figure) get passed to the cognitive processing algorithms, which perform calculations using this information.

\subsubsection{Agent movement}

Objects in the PCAI move by updating their orientation and position variables (IMPLEMENT in Algorithm 1) and any other agent properties that the modeller wishes to update at each timestep. The Breve simulation engine then renders the object graphically on the screen based on these new values. Generally speaking, objects also have a velocity associated with them for each timestep, which is used to calculate their new position. The PCAI does not impose specific movement restrictions on objects, so it is expected that modellers will restrict the movement of 
their agents and other simulation objects in ways that reflect actual movement restrictions on these objects (CORRECT in Algorithm 1).

\subsection{Gathering data on $D$. rerio behaviour and properties}

As stated above, to demonstrate how the PCA framework can be used to create species-specific models, an species and environment specific $D$. rerio aggregate model was created, using the PCAI, in conjunction with data gathered on $D$. rerio movement and behaviour. This section will describe the data gathering and data analysis process. It will begin by presenting data gathering techniques for individual and small group D. rerio movement and behaviour. It will then describe the analysis techniques used to process the data and, finally, the results obtained from the analysis of this data.

\subsubsection{Methodology}

As argued in Chapter 3, in order to be considered a species-specific PCA framework model, the D. rerio model needed to incorporate, as much as possible, empirical data regarding perceptual, cognitive and movement abilities of $D$. rerio individuals. To do this, information was gathered on movement abilities (speed and turning angle), cognitive-behavioural properties (preferred distance from other $D$. rerio), perceptual properties (eye position, angle of vision, lateral line position) and physical characteristics (body size and shape) of individuals from a group of $18 \mathrm{D}$. rerio. The methodology for collecting this data is described below.

Speed, turning angle and preferred distance $D$. rerio movement data were gathered from individual $D$. rerios swimming freely in an uncovered $60 \times 46 \times 90 \mathrm{~cm} 246$ litre glass tank lined with white Coroplast on four of the five sides (bringing the actual dimensions to $50 \times 45 \times 89$ $\mathrm{cm}$ ) (see Figure 16). Fish movements were recorded using two Sony DCR-HC96 handycams positioned on tripods in front of the tank. For individual trials 12 fish were placed one at a time into the trial tank and behaviour recorded for 10 minutes. The first 5 minutes of this time was considered a settling in period. Movement data were analysed for the last 5 minutes of the trial. 
$D$. rerio behavioural data was gathered from small groups of $4 D$. rerios using a similar approach for the groups of 4 fish (four groups of 4 , for a total of 16 fish). At the end of each trial, the fish used in the trial were placed in a holding tank to avoid repeat use of the same individual or individuals.

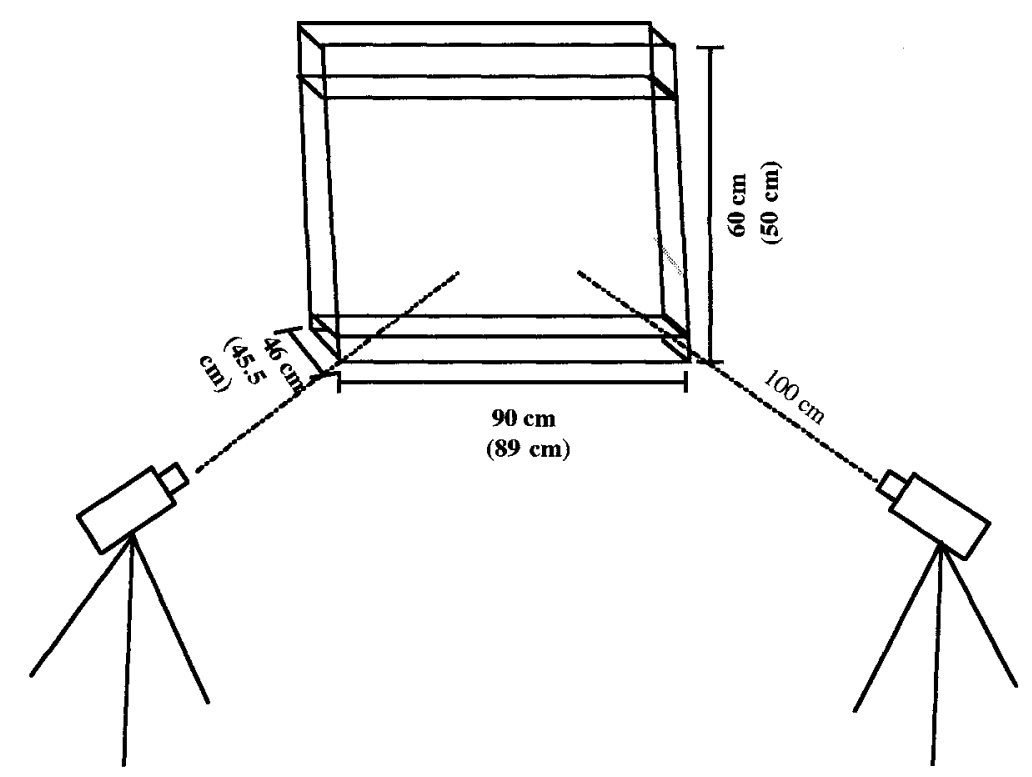

Figure 16: Tracker 3D aquarium set up.

Analysis of the fish video data was carried out using the Tracker 3D software and preprocessing macros developed by Grünbaum et al. (2005). Raw digital video for each camera was imported into a 2 processor, dual core Mac Pro (approximately $100 \mathrm{~GB}$ of data was imported and processed). The video was converted into QuickTime movies and pre-processed using ImageJ, an image processing and analysis program. The Tracker 3D program further processed the resulting data, generating position and trail data for each fish in each frame of the video.

Not all of the video shot during the trials resulted in processable video or usable data. In some cases the Tracker 3D program failed to produce any data from a frame and in other cases the data produced was clearly inaccurate (e.g., showing multiple fish when only one fish was present, disconnected trails across frames, showing fish moving $15 \mathrm{~cm}$ in $1 / 30^{\text {th }}$ of a second when most data showed fish moving at a maximum speed closer to $3 \mathrm{~cm}$ in $1 / 30^{\text {th }}$ of a second). However, the large number of video frames available for analysis ( 9000 frames 
for each individual fish, with 108000 frames in total across the 12 individuals chosen for observation) allowed for a random sampling approach to the data analysis. Specifically, the total number of frames for each fish was divided into 15 groups of 600 frames (with the number of groups chosen based on computer memory requirements). These groups were then analysed in a random order, with 50 physically possible coordinate data points for the fish being considered the minimum number to consider a group usable. Data for individuals (and groups of 4 , see below) were manually reviewed to ensure accurate path construction and data collection. Ultimately, sufficient quantities (50 or more points) of usable individual movement data were successfully gathered for 7 of the 12 observed individuals from the individual movement trials $(39 \%$ of the total population of $18 \mathrm{D}$. rerio being modelled).

A similar approach was taken for analysis of the groups of four $D$. rerio. In this case, only data that provided information about the position of each of the four fish in a particular frame was considered valid (i.e., frames that provided position data for only 3 or fewer of the $4 D$. rerio in a group of 4 were not considered). Again, the total data captured for a particular group of four fish ( 9000 frames) was divided into 15 smaller groups of 600 frames, with groups being analysed in a random order. A frame group was considered an adequate data source if there were at least 20 frames in the group providing data on each of the four fish ( 20 frames were sufficient to provide 480 data points on inter-fish distance). Data were successfully gathered for all four of the groups observed (89\% of the total population being modelled).

For the individual trials, the raw positional data was further analysed in order to obtain speed measures of each fish for each $1 / 30^{\text {th }}$ of a second interval (position data in two adjacent frames provided one speed measurement). Then the average speed for each individual, as well as average speed across all fish, and the maximum and minimum speeds within and across fish were calculated.

Position data was also used to calculate turning angle for fish (position data in three adjacent frames provided one turning angle measure). Then average, maximum and minimum angle between vectors were calculated in order to determine the ability of the $D$. rerio to change direction over time. Again, average, maximum and minimum values were calculated within and across fish. 
Distance between fish at each point in time (average, maximum and minimum) was calculated for the fish in groups of four. With respect to inter-fish distance, for each fish, the distance from that fish to the fish closest to it, the distance from that fish to the fish farthest from it and the average distance of that fish to the other three fish was calculated and reported.

Perception Vision and lateral line senses are generally recognised as the two senses most active in fish aggregation (Partridge and Pitcher, 1980) so empirical data was gathered on both of these senses. Following the methodology of Protasov (1970), the angle of the visual field of the $D$. rerio was established based on the physical structure and position of the eye. This was determined using measurements of anatomical data from the VCCRI Fishnet database (Bryson-Richardson et al., 2007) (see Figure 17). The position of the eye relative to the rest of the body was determined by measurements of $2 D$. rerio specimens taken from the same tank as the school of 18 being studied (but not from the group of 18). Lateral line perceptual field positions were based on knowledge of lateral lines in $D$. rerio larvae (Whitfield et al., 1996; Van Trump and McHenry, 2008), combined with information that as D. rerio age, their lateral line neurons increase in number, with even spacing and along the same line (Moorman 2001). Position on adult D. rerio was estimated using two $D$. rerio specimens.

Acuity of the visual and lateral line perceptual fields was not directly determined. Assuming fish were not able to perceive objects outside of the experimental tank with any significant acuity, the maximum possible perceptual field extent for any perceptions would necessarily be limited to $89 \mathrm{~cm}$, the largest dimension of the experimental tank.

Size and shape Length, width and shape of the $D$. rerio in the school being studied were based on measurements of two specimens taken from the same tank as the school of 18 being studied. Measurements were taken for height, width and length (standard length and total length).

\subsubsection{Results}

Speed and turning angle The results of the movement data analysis for individuals are presented in Tables 1 and 2. Table 1 shows speed data for the seven individual fish and Table 2 
shows turning angle data for these same fish. Generally speaking, for both average speed and average turning angle, deviation compared both across all fish and compared between fish was low. Maximum and minimum values showed greater between-fish differences.

\begin{tabular}{lllll} 
Fish & $\bar{s}$ & $s_{\min }$ & $s_{\max }$ & $n$ \\
\hline 1 & $0.48 \pm 0.07$ & 0.06 & 1.3 & 58 \\
2 & $0.38 \pm 0.05$ & 0.01 & 1.52 & 350 \\
3 & $0.31 \pm 0.05$ & 0.03 & 1.17 & 137 \\
4 & $0.42 \pm 0.06$ & 0.05 & 1.92 & 320 \\
5 & $0.49 \pm 0.07$ & 0.06 & 2.09 & 211 \\
6 & $0.53 \pm 0.17$ & 0.01 & 2.73 & 259 \\
7 & $0.37 \pm 0.08$ & 0.03 & 2.47 & 270 \\
\hline$\overline{\text { ind }}$ & $0.43 \pm 0.005$ & $0.036 \pm 0.021$ & $1.89 \pm 0.59$ & 229 \\
all & $0.42 \pm 0.08$ & 0.01 & 2.73 & 1605
\end{tabular}

Table 1: Speed data for D. rerio, within fish and across all fish: For each fish, the average speed $(\bar{s})$, minimum speed $\left(s_{\min }\right)$ and maximum speed $\left(s_{\max }\right)$ were calculated (in $\mathrm{cm} / 30^{\text {th }}$ of a second), using available number of data points for each fish $(n)$. Then, the average of the individuals values $(\overline{i n d})$ and values for data pooled across all fish (all) were calculated.

\begin{tabular}{lllll} 
Fish & $\bar{\theta}$ & $\left(\theta_{\min }\right)$ & $\theta_{\max }$ & $n$ \\
\hline 1 & $1.10 \pm 0.92$ & 0.1 & 3.04 & 57 \\
2 & $1.19 \pm 0.65$ & 0 & 3.07 & 340 \\
3 & $1.33 \pm 0.88$ & 0 & 3.07 & 133 \\
4 & $1.17 \pm 0.69$ & 0 & 3.12 & 294 \\
5 & $0.99 \pm 0.52$ & 0 & 2.96 & 200 \\
6 & $1.14 \pm 0.63$ & 0 & 3.11 & 239 \\
7 & $1.11 \pm 0.70$ & 0 & 3.07 & 263 \\
\hline$\overline{\text { ind }}$ & $1.15 \pm 0.01$ & $0.009 \pm 0.038$ & $3.06 \pm 0.05$ & 218 \\
all & $1.15 \pm 0.68$ & 0 & 3.12 & 1526
\end{tabular}

Table 2: Turning angle data for D. rerio, within fish and across all fish: For each fish the mean turning angle $(\bar{\theta})$, the minimum turning angle $\left(\theta_{\min }\right)$, the maximum turning angle $\left(\theta_{\max }\right)$ were calculated in radians, using the available number of data points for each fish $(n)$. Then, the average of the individuals values $(\overline{\text { ind }})$ and values for data pooled across all fish (all)were calculated.

Preferred distance There was high variability in the average values for closest fish, farthest fish and average fish distance (see Table 3). Average distance maintained across fish was 
$10.54 \mathrm{~cm}$, although the variability was high. The mean closest fish distance $(6.07 \mathrm{~cm})$ was relatively close to this overall mean distance value, and again, variability was quite high across fish.

\begin{tabular}{llll}
\hline Group & close & fär & dist \\
1 & $9.39 \pm 5.05$ & $29.09 \pm 6.47$ & $19.68 \pm 4.18$ \\
2 & $4.80 \pm 3.27$ & $10.16 \pm 3.39$ & $6.97 \pm 2.95$ \\
3 & $4.76 \pm 3.28$ & $9.43 \pm 4.58$ & $6.86 \pm 3.16$ \\
4 & $4.72 \pm 2.26$ & $10.99 \pm 3.91$ & $7.64 \pm 2.41$ \\
grōup & $5.92 \pm 2.32$ & $14.92 \pm 9.47$ & $10.30 \pm 6.27$ \\
all & $6.07 \pm 4.34$ & $15.20 \pm 10.02$ & $10.54 \pm 6.66$ \\
\hline $\min _{\text {all }}$ & 0.88 & 5.08 & 3.66 \\
$\max _{\text {all }}$ & 23.13 & 42.13 & 29.52
\end{tabular}

Table 3: A summary of the individual distance data for fish moving in groups of four: Average distance from a fish to its nearest neighbour in the group over time (close), mean distance from a fish to its farthest neighbour in the group over time ( $f \bar{a}$ ), the across group mean of the mean distance of a fish to its neighbours in the group over time (dist). Then the mean of group values ( $g r \bar{o} u p$ ) and the results for values pooled across fish (all), as well as the minimum across all fish $\left(\min _{\text {all }}\right)$ and the maximum across all fish ( $\left.\max _{a l l}\right)$.

Body size and shape Body size measurements for two $D$. rerio specimens are presented in Table 4.

\begin{tabular}{llllll} 
Specimen & Width & Length (T,S) & Height & Eye Position & Lateral Line Position \\
\hline 1 & 3.5 & $31.2,24.9$ & 5.3 & $1.8,1.6$ & $3.3,1.5$ \\
2 & 5.4 & $33.4,27.1$ & 6 & $2.2,1.9$ & $3.7,2.3$ \\
mean & 4.5 & $32.3,26,0$ & 5.6 & $2.0,1.8$ & $3.5,1.9$
\end{tabular}

Table 4: Measurements of $D$. rerio size: Width $(\mathrm{mm})$ at the widest part of the fish, length $(\mathrm{mm})$ both total (T) and standard (S), height $(\mathrm{mm})$ at the highest part of the fish, eye position from the nose in and the bottom up, and estimated lateral line position $(\mathrm{mm})$ from the top and bottom at the highest point of the fish.

Perception Based on measurements taken from Fishnet database resonance imaging photographs in the Zebrafish Database (Bryson-Richardson et al. 2007), the angle of the visual field was 13 degrees off of the normal, and 180 degrees wide (see Figure 17). Eye position was 


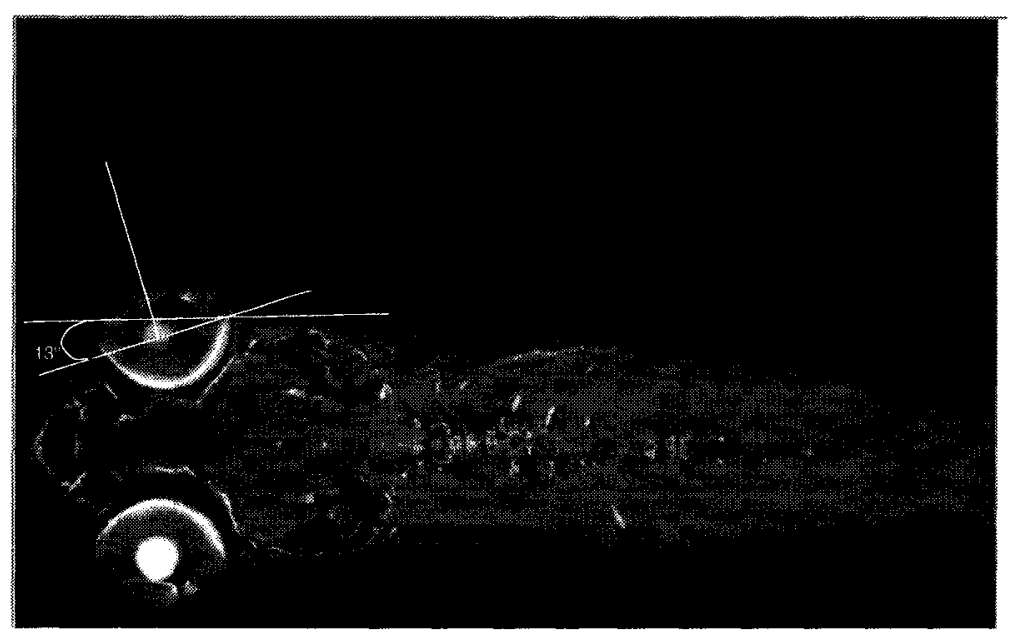

Figure 17: A coronal scan of a $17 \mathrm{~mm} D$. rerio, used to measure D. rerio eye position and angle (Bryson-Richardson et al, 2007).

measured on two $D$. rerio. The mean eye position was $0.20 \mathrm{~cm}$ from the tip of the nose and 0.18 $\mathrm{cm}$ up from the bottom of the head (ratio 1:1.84).

Data on lateral line positions in $D$. rerio larvae (Whitfield et al, 1996; Van Trump and McHenry 2007 ) indicate that $D$. rerio lateral line fields run along both sides of the fish from the head behind the eye to the base of the tail, slightly above the midline of the fish. A visual inspection of two adult $D$. rerio specimens placed these lines an average estimated $1.9 \mathrm{~mm}$ from the top of the fish and $3.5 \mathrm{~mm}$ from the bottom of the fish (ratio 1:1.84) (see Figure 18).

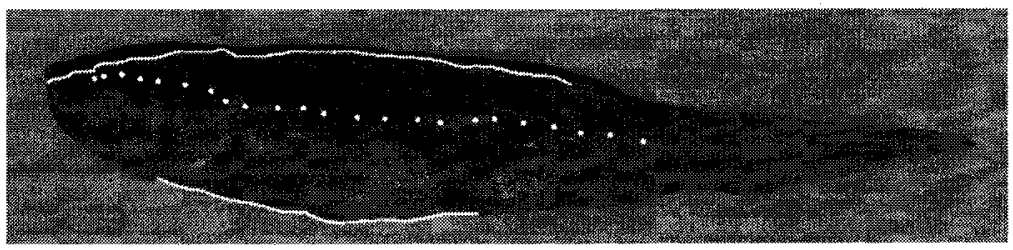

Figure 18: Estimated D. rerio lateral line position (white dots superimposed on photograph, with white lines indicating body position, not including shadow), based on lateral line position in larvae (as reported by Whitfield et al, 1996 and Van Trump and McHenry, 2008). 


\subsection{Creating an IBSEM model of $D$. rerio aggregation}

Turning to the model, this section will discuss the construction of the PCA $D$. rerio model agents, with a focus on how the collected data were incorporated into the model. The $D$. rerio aggregate model was created using the PCAI, as described in Section 4.1. Available data about the physical shape and movement abilities of the $D$. rerio, types, visual and lateral line ranges and information processing abilities of the perception of the $D$. rerio were incorporated into the model. Specific details of this incorporation, as well as agent implementation and agent behavioural algorithms, are discussed below.

\subsubsection{Agent behaviour during one timestep}

Agent behaviour in a single timestep of the $D$. rerio model is described in Algorithms 1 and 2 . Algorithm 2 describes functions specific to the $D$. rerio model behaviour. These functions are called by functions within the timestep function in Algorithm 1 and represent one possible instantiation of the more general behaviour in a single timestep described in Algorithm 1. These functions are further explained in Sections 4.3.3, 4.3.4 and 4.3.5.

\subsubsection{Physical shape of agents}

Agents were composed of five spheres - 4 main body spheres, two with diameter $0.50 \mathrm{~cm}$, two with diameter $0.75 \mathrm{~cm}$ and one very small sphere placed above the head sphere to visibly indicate agent orientation (see Figure 19). The total length of the agent was $2.50 \mathrm{~cm}$, consistent with the observed length of $D$. rerio (mean standard length measured being $2.60 \mathrm{~cm}$ ). The use of spheres meant that the maximum width of the agent was $0.75 \mathrm{~cm}$, somewhat wider than the mean width measured $(0.45 \mathrm{~cm})$. The height of the agent was also $0.75 \mathrm{~cm}$, slightly larger than the height of the two specimens measured $(0.56 \mathrm{~cm})$.

\subsubsection{Perceptual fields and resolution}

Both visual and lateral line fields of the $D$. rerio were represented in the Breve environment as cones extending outward from the body of the agent (see Figure 20). The visual fields of the 


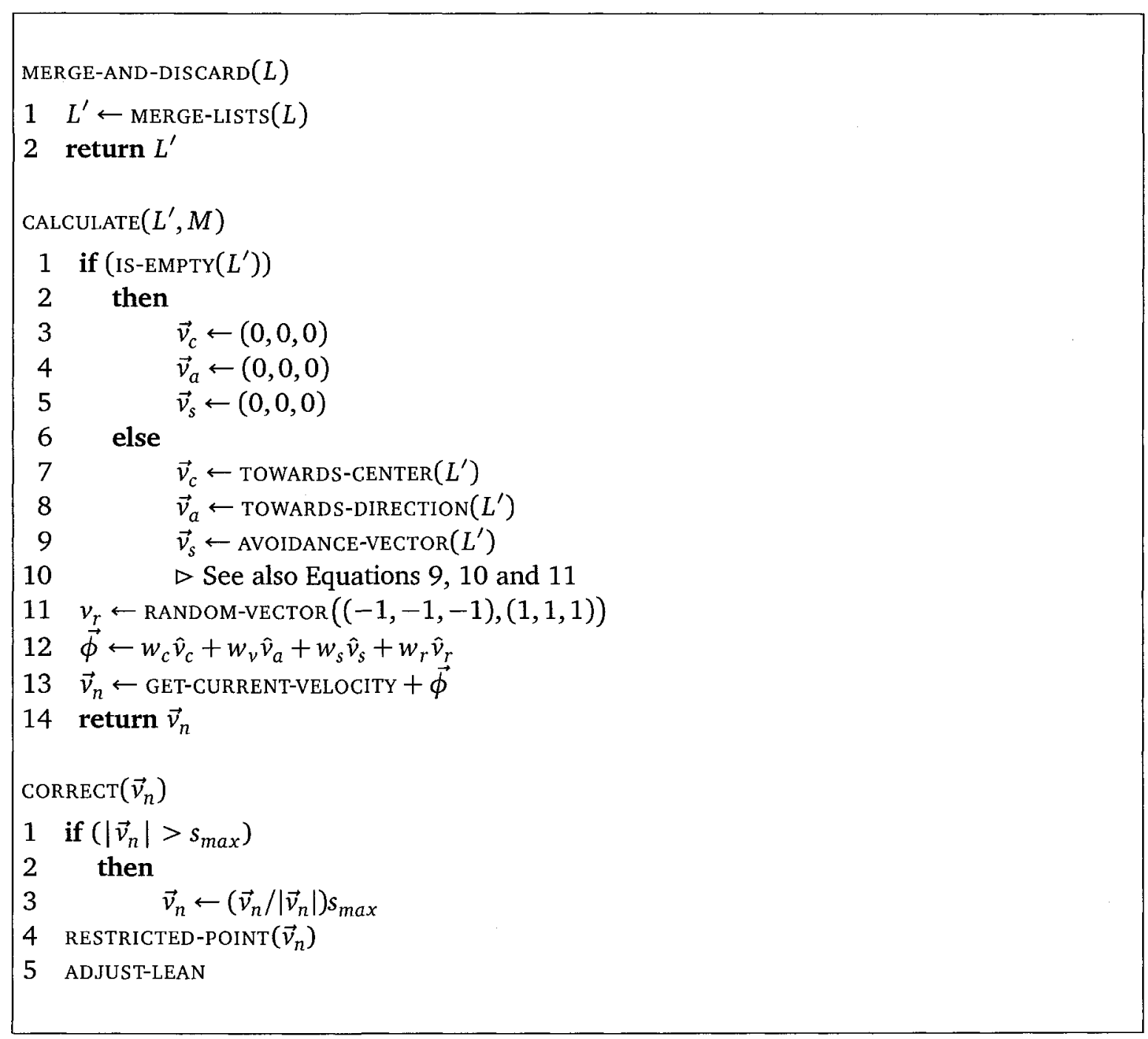

Algorithm 2: $D$. rerio agent behaviour during one timestep. 

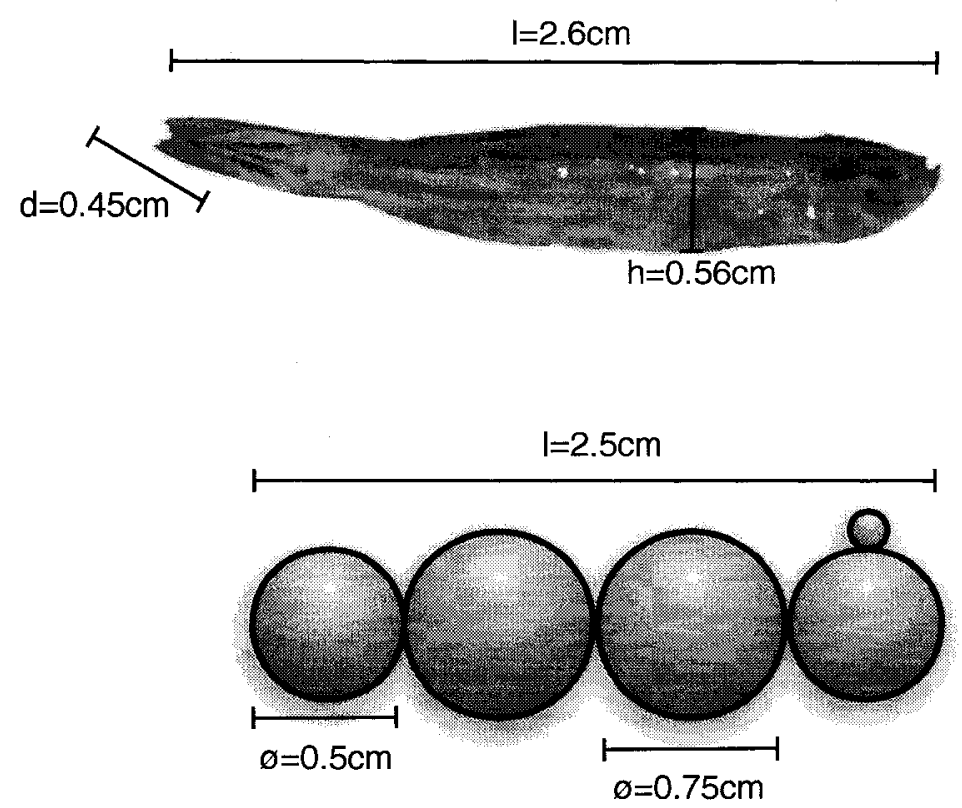

Figure 19: Agent shape, compared with $D$. rerio shape. The agent is composed of two 0.75 diameter $(\emptyset)$ spheres, two $0.5 \mathrm{~cm}$ diameter spheres and one small sphere that indicates orientation (drawing not to scale).

agents were represented by two half spheres (cones with a 180 degree angle at the vertex), one on each side of the agent. Based on the data gathered on $D$. rerio vision (see Section 4.2.2), the angle of the field was set to be 13 degrees off of the normal, and 180 degrees wide. in local (agent-centered) coordinates, the vertices of the cones were located at $(0,0,0.25)$ and $(0,0,-0.25)$ respectively (the cones extended outwards on either side of the 'head sphere' of the agent). In the absence of specific data concerning $D$. rerio visual acuity, the length of the visual fields of model agents was set to $40 \mathrm{~cm}$. Since it was assumed that the visual field of the actual fish would not extend usefully through the glass of the tank, and since the tank was $50 \times 45 \times 89$ $\mathrm{cm}$, this was considered to be a conservative approximation of the visual field extent for the fish being modelled. Lateral line fields were set at three points on both sides of the zebra fish agent, from tail to head, for a total of 6 fields (see Figure 20). The vertex of each field was placed at the centre of its respective body sphere. It was hypothesised that lateral lines, because they respond to water flow (Van Trump and McHenry, 2008), operate most effectively from an information processing perspective at relatively short distances. Therefore the lateral line fields were set to extend $5 \mathrm{~cm}$ outwards from the body of the agent. 
In the absence of data on the perceptual resolution of $D$. rerio vision and the lateral line, some assumptions were made about the data that these perceptions could supply to the agents. It was assumed that $D$. rerio vision was accurate enough to return information about the position and velocity (speed and direction of movement) of other fish in the tank. A similar assumption was made for the lateral line fields. In the case of the lateral line fields, this was considered plausible when taken in conjunction with the relatively short range of the lateral line fields. It is possible that lateral line fields can also detect data at greater ranges (e.g., presence of another object, if not its precise location, or velocity) but this was not modelled in this case. Thus, when either a visual or lateral line cone of an agent intersected any of the spheres representing the body of another agent (as determined in the INTERSECT function), a perceptual resolution tuple, $\tau=\langle\vec{\rho}, \vec{v}\rangle$ was returned. This tuple provided the observing agent with a position coordinate $(\vec{\rho})$ and movement vector $(\vec{v})$ for the intersected agent, resulting in a set, $R_{F, t}$, of tuples for each field, $F$, at that time, $t$. The set of all $R_{F, t}$ is denoted by $L$.

\subsubsection{Cognitive algorithms for determining movement}

There is no experimental data currently available on the specific cognitive processes $D$. rerio use in order to aggregate. However, as noted by Parr (1927) the necessary conditions for aggregation would seem to be an attraction to other agents, in order for the aggregate to have cohesion, and repulsion from other agents, so that the aggregate does not collapse into a single point. Consequently, for this model; a Breve version of Reynold's attraction and repulsion algorithm (Klein 2002; Reynolds 1987) was adapted to incorporate the reception of information from multiple perceptions. The attention and cognitive algorithms are described below.

Attention $D$. rerio agents can receive information about another agent through both their visual and lateral line fields (see Figure 20). Different types of information (e.g., position, velocity) about the same agent are stored in a single tuple, because it is assumed that the $D$. rerio has successfully solved this aspect of the binding problem. Since it is not currently known how, or even if, $D$. rerio has solved the binding problem with respect to different senses returning information about the same object, it was decided that at the attentional stage, all received 


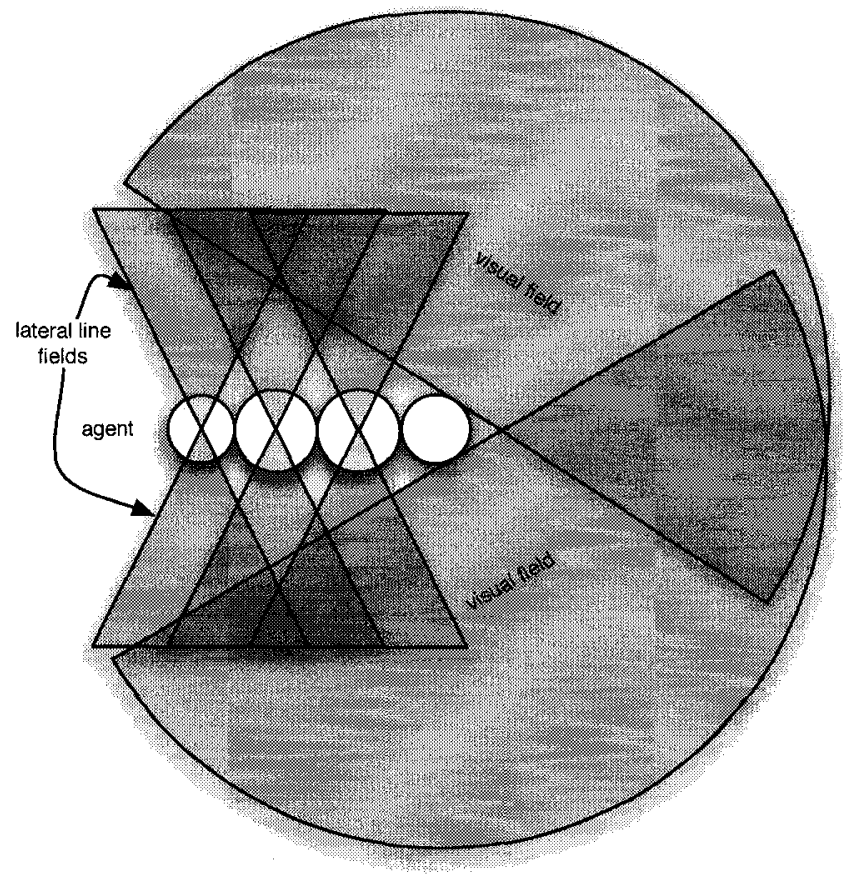

Figure 20: Visual and lateral line fields of the agent (field size not to scale). 
information, $L$, would be merged into a single list, $L^{\prime}$, with the result that duplicate information (tuples) could be in the list if the same object was perceived by multiple fields. The practical effect of leaving multiple copies of information about the same agent in the list of agent data in the input to the cognitive algorithm was to increase the influence of agents that are detected by multiple perceptions, because their information is effectively weighted more heavily. This effect is gained with no additional processing and could be advantageous to the aggregating animal, since animals detected by multiple perceptions may in fact be more relevant to the aggregating animal.

Cognition As in Reynolds' model, agents use the information about position and velocity of other agents to calculate preferred movement vectors: $\vec{v}_{c}$ (the centring vector), $\vec{v}_{a}$ (the velocity alignment vector) and $\vec{v}_{s}$ (the spacing vector) based on attraction to the centre of the perceived agents, alignment with the velocity of the group, and an avoidance of those agents close to the perceiving agent. Specifically, for agent $\alpha_{i}$ at time $t$, where there are $n$ perceived agents,

$$
\vec{v}_{c}=\frac{\sum_{j=0}^{n}\left(\vec{\rho}_{j}(t)-\vec{\rho}_{i}(t)\right)}{n}
$$

and

$$
\vec{v}_{a}=\frac{\sum_{j=0}^{n}\left(\vec{v}_{j}(t)-\vec{v}_{i}(t)\right)}{n}
$$

The result is a centring vector, $\vec{v}_{c}$, that points towards the centre of the group of perceived agents, and an alignment vector, $\vec{v}_{a}$, that points towards the average direction of movement of the group of perceived agents. It is generally assumed in aggregate modelling that agents are aligned with (i.e., point towards) their direction of movement, and this is the case in this model as well. In Algorithm 2 these equations are represented by the TOWARDS-CENTRE $\left(L^{\prime}\right)$ and TOWARDS-DIRECTION $\left(L^{\prime}\right)$ functions. 
To determine the spacing vector, a subgroup of the agents, $\delta$, is constructed that consists of all agents who are within (closer or equal to) a preferred spacing, space ${ }_{\text {pref }}$, from the agent. Then, for all $m$ of these agents,

$$
v_{s}=-\frac{\sum_{j=0}^{m}\left(\vec{\rho}_{j}(t)-\vec{\rho}_{i}(t)\right)}{m}
$$

In Algorithm 2 this equation is represented by the AVOIDANCE-VECTOR $\left(L^{\prime}\right)$ function.

These movement vectors are combined, along with a random vector influence, $v_{r}$, that represents natural variability in movement, to give a final acceleration vector, $\vec{\phi}$ with the contribution of each vector being influenced by a weight value associated with each vector (the centring weight $\left(w_{c}\right)$, velocity weight $\left(w_{v}\right)$, spacing weight $\left(w_{s}\right)$ and random weight $\left.\left(w_{r}\right)\right)$ as follows:

$$
\vec{\phi}=w_{c} \hat{v}_{c}+w_{v} \hat{v}_{a}+w_{s} \hat{v}_{s}+w_{r} \hat{v}_{r}
$$

One cognitive parameter of the model which could be set based on experimental data was the distance at which the agent would consider itself to be too close to another agent, space pref $_{\text {. }}$ In this case, data were available on the minimum space D. rerio allowed between themselves and other D. rerio in their group (a mean across frames of $6.07 \mathrm{~cm}$ ) and the mean space between themselves and other D. rerio in the group (a mean across frames of $10.54 \mathrm{~cm}$ ). Either would be a possible value for space $_{\text {pref }}$, so model results were analysed for both values of the spacing parameter.

The other cognitive model parameters associated with cognition are the vector weights, $\left(w_{c}\right.$, $w_{v}, w_{s}$ ), that determine how much of a contribution the centring, velocity and spacing vectors have on behaviour. Given that there is no experimental evidence regarding the cognitive process of aggregation, it is difficult to say, even assuming that D. rerio are using a cognitive algorithm comparable to Reynolds, how much weight they are giving to these vectors. Consequently, two different values for each of these parameters were selected (see Table 5 for values) and the results analysed for each of the resulting parameter combinations (see Table 6). 


\subsubsection{Movement}

The maximum speed recorded for an individual D. rerio $\left(2.73 \mathrm{~cm} / 30^{\text {th }}\right.$ of a second) was used to set the maximum speed of agents in the model. The maximum turning angle of agents in the model $\left(3.0 \mathrm{~cm} / 30^{\text {th }}\right.$ of a second) was set based on the maximum turning angle recorded for an individual. As well, agents were prevented from swimming upside down or on their sides, as textitD. rerio only swim upside or on their sides when they suffer a loss of attitude control due to illness or injury.

Movement restrictions were placed on the fish at the end of the cognitive stage, once a preferred vector had been calculated by the cognitive algorithm (see the CORRECT function in Algorithm 2). The assumption was made that the fish would calculate the most desirable movement vector and attempt to move based on this vector. However, the end movement would be reduced by the constraints discussed above. If the size of the desired movement vector exceeded the maximum speed of the fish $\left(s_{\max }\right)$, the length of movement vector was reduced to the maximum speed. If the turn required by the desired movement vector was greater than the maximum turning angle $\left(t \theta_{\max }\right)$, the vector was recalculated in order to restrict the fish from turning at an angle greater than $t \theta_{\max }$ (RESTRICTED-POINT in Algorithm 2, not shown). Restrictions against swimming upside down or on the side were also implemented, such that if the angle would have resulted in an excessive tilt (either side to side or front to back), the angle was reset to be no more than 90 degrees in these directions (ADJUST-LEAN in Algorithm 2, not shown).

\begin{tabular}{ll} 
Parameter & Value $(\mathrm{s})$ \\
\hline Maximum speed $\left(s_{\max }\right)$ & 2.73 (from speed data) \\
Maximum turning angle $\left(t \theta_{\max }\right)$ & 3.10 (from angle data) \\
Preferred space $\left(\right.$ space $\left.e_{\text {pref }}\right)$ & 6.07 (average minimum distance), 10.54 (average distance) \\
Centring weight $\left(w_{c}\right)$ & 1,3 \\
Velocity weight $\left(w_{v}\right)$ & 1,3 \\
Spacing weight $\left(w_{s}\right)$ & 1,3
\end{tabular}

Table 5: Parameters used to investigate model behaviour. When multiple values for the same parameter were considered, all values are listed. All combinations of all parameter values considered result in 16 parameter combinations in total (see Table 6). 


\begin{tabular}{ll} 
Parameter Combination & $\begin{array}{l}\text { Shorthand Notation } \\
\left(s_{\max }, t \theta_{\max }, \text { space }_{\text {pref }}, w_{c}, w_{\nu} w_{s}\right)\end{array}$ \\
\hline$\left(\right.$ space $_{\text {pref }}$ (rounded), $\left.w_{c}, w_{\nu} w_{s}\right)$ \\
\hline$(2.73,3.1,6.07,1,1,1)$ & $(6,1,1,1)$ \\
$(2.73,3.1,6.07,1,3,1)$ & $(6,1,3,1)$ \\
$(2.73,3.1,6.07,1,3,3)$ & $(6,1,1,3)$ \\
$(2.73,3.1,6.07,3,1,1)$ & $(6,1,3,3)$ \\
$(2.73,3.1,6.07,3,1,3)$ & $(6,3,1,1)$ \\
$(2.73,3.1,6.07,3,3,1)$ & $(6,3,1,3)$ \\
$(2.73,3.1,6.07,3,3,3)$ & $(6,3,3,1)$ \\
$(2.73,3.1,10.54,1,1,1)$ & $(6,3,3,3)$ \\
$(2.73,3.1,10.54,1,3,1)$ & $(10,1,1,1)$ \\
$(2.73,3.1,10.54,1,1,3)$ & $(10,1,3,1)$ \\
$(2.73,3.1,10.54,1,3,3)$ & $(10,1,1,3)$ \\
$(2.73,3.1,10.54,3,1,1)$ & $(10,1,3,3)$ \\
$(2.73,3.1,10.54,3,1,3)$ & $(10,1,1,1)$ \\
$(2.73,3.1,10.54,3,3,1)$ & $(10,1,1,1)$ \\
$(2.73,3.1,10.54,3,3,3)$ & $(10,1,1,1)$ \\
& $(10,1,1,1)$
\end{tabular}

Table 6: A list of the 16 parameter combinations, in the following order (maximum speed $\left(s_{\max }\right.$ ), maximum turning angle $\left(t \theta_{\max }\right)$, preferred space $\left(\right.$ space $\left._{\text {pref }}\right)$, centring weight $\left(w_{c}\right)$, velocity weight $\left(w_{v}\right)$, spacing weight $\left(w_{s}\right)$. A shorthand label for each combination is included for easy reference in the rest of the chapter. 


\subsection{Model analysis, results and predictions}

The simulation was run with 18 agents for each of the 16 parameter combinations of parameter values. Each parameter combination was run 35 times, for 500 timesteps each, with agents initially positioned at $(0,0,0)$. Agents were not restricted with respect to where they could move through space (i.e., there was infinite space available in the model). Measures of aggregate behaviour- aggregate speed, aggregate turning angle and aggregate simple volume (described in Table 7) - were quantitatively analysed across multiple parameter combinations. The data were also analysed for qualitative aggregate phenomena that might be observable in the actual D. rerio aggregate.

\subsubsection{Analysis}

Aggregate measures used for the models in this thesis (models in Chapters 4, 5 and 6), are listed in Table 7. As noted in the table, some of these are common aggregate measures used by other modellers. Others were developed specifically for the D. rerio model and the other models in Chapters 5 and 6.

For this model, stragglers were defined as agents not within 20 units of any other agent and groups were sets of all agents that were within 20 units of at least one other agent already in the group. A cluster was defined as either a group or an individual (i.e., a model that at timestep $t$ had one group and one individual would have two clusters). Like the practical definition of aggregation discussed in Chapter 2, these measures are based on visibly measurable properties of the aggregate, although, as will be discussed in Chapter 5, a less ad hoc definition might be based on the information possessed by each agent. For this model, 20 units was considered to be a relatively conservative estimate of aggregation, and a compromise between agents having perceptual fields extending 40 units in some directions, lateral line fields extending only 5 units, and the agents having some blind spots.

For each run of each parameter combination (see Figure 21), the maximum, minimum and mean values of the aggregate measure across the last 450 (out of 500) timesteps of the model were calculated, for each aggregate measure. These maximum and minimum values were then 


\begin{tabular}{|c|c|}
\hline Measure name & Description \\
\hline simple volume $\left(v_{\text {simp }}\right)$ & $\begin{array}{l}\text { Volume of bounding box calculated using maximum and mini- } \\
\text { mum } x, y \text { and } z \text { values across all individuals. This provides an } \\
\text { approximation of aggregate volume. It will generally over es- } \\
\text { timate volume. Can be used to calculate aggregate density by } \\
\text { dividing by the number of individuals in the aggregate (used } \\
\text { by Hemelrijk and Hildenbrandt, 2007). }\end{array}$ \\
\hline expanse $(e x p)$ & $\begin{array}{l}\text { A measure of aggregate size, which is the root-mean-square } \\
\text { distance from individuals to the center of the aggregate. This } \\
\text { provides a less approximate measure of the size of the aggre- } \\
\text { gate, but is difficult to directly relate to the density or volume } \\
\text { of the aggregate (used by Parrish et al., 2002; Huth and Wisel, } \\
\text { 1992). }\end{array}$ \\
\hline polarisation $(p)$ & $\begin{array}{l}\text { Sum of angles between individual velocity and mean velocity, } \\
\text { divided by number of individuals. A larger value indicates } \\
\text { less alignment within the aggregate (used by Huth and Wissel } \\
\text { 1992; Parrish et al. 2002). }\end{array}$ \\
\hline Number of groups ( $n_{\text {groups }}$ ) & $\begin{array}{l}\text { A group is a collection of individuals within a certain distance } \\
\text { from each other, as determined by the researcher (used by } \\
\text { Parrish et al., 2002). }\end{array}$ \\
\hline Number of stragglers $\left(n_{\text {strag }}\right)$ & $\begin{array}{l}\text { Stragglers are individuals who are not within a certain distance } \\
\text { of any other individual (used by Parrish et al., 2002). }\end{array}$ \\
\hline Number of clusters ( $n_{\text {clust }}$ ) & $\begin{array}{l}\text { Number of groups plus number of stragglers (used by current } \\
\text { analysis). }\end{array}$ \\
\hline $\begin{array}{l}\text { aggregate distance/aggregate } \\
\text { speed }\left(s_{a g g}\right)\end{array}$ & $\begin{array}{l}\text { How far the centre of the group moves each time step (used } \\
\text { by Warburton and Lazarus, 1991; Viscido et al., 2007). }\end{array}$ \\
\hline aggregate turning angle $\left(t \theta_{\text {agg }}\right)$ & $\begin{array}{l}\text { The angle of movement of the centre of the group each } \\
\text { timestep (used by current analysis) }\end{array}$ \\
\hline
\end{tabular}

Table 7: Description of aggregate measures 
averaged across all 35 runs of the model having a particular parameter combination in order to obtain a single average maximum value, a single average minimum value and a single mean value representative of the value of the aggregate measure for that parameter combination.

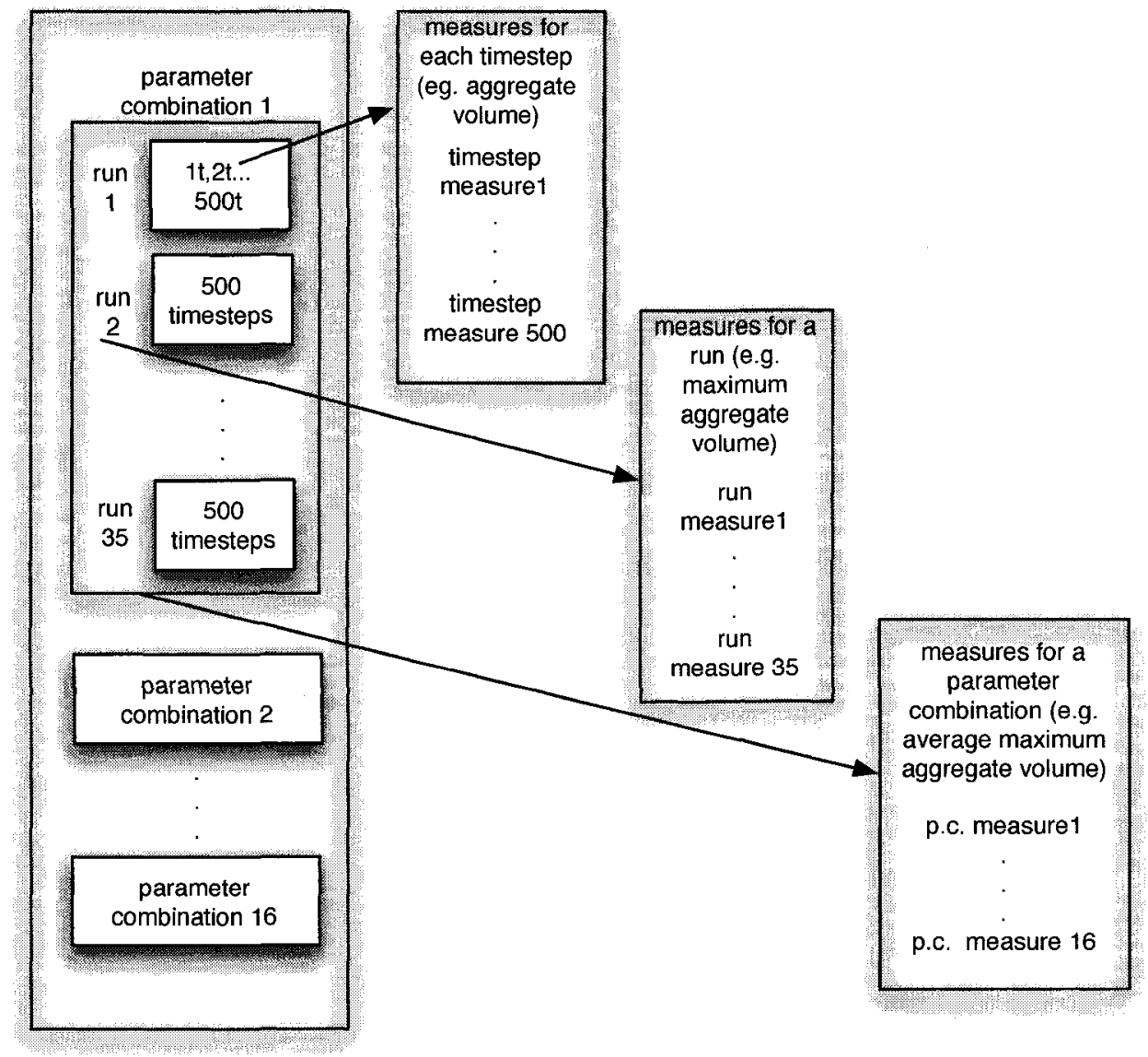

Figure 21: An explanation of how aggregate measures are generated. Measures are generated at multiple levels - the timestep, the run and the parameter combination. Generally speaking, timestep measures are used to calculate run measures and run measures are used to calculate parameter combination measures.

\subsubsection{Results and predictions}

Group size remained 1 for all model runs, with all parameter combinations. Specific aggregate results for group volume, polarisation, group speed and group angle will be discussed below, with a focus on similarities of results across parameter combinations, and a summary across combinations. See Appendix A for the results from all parameter combinations and Table 8 for a summary of physically plausible results (as discussed under Group Volume, below). Results were 
used to make predictions about the aggregate behaviour of the group of $18 \mathrm{D}$. rerio and group speed, group angle and group volume were directly compared with data from the behaviour of the aggregate of $18 \mathrm{D}$. rerio.

\begin{tabular}{llll} 
measure & range $_{\max }$ & range $_{a v r}$ & range $_{\min }$ \\
\hline$v_{\text {simp }}$ & $52.41-1536.43$ & $27.90-1049.62$ & $7.54-712.02$ \\
$p$ & $0.39-1.50$ & $0.19-0.87$ & $0.11-0.457$ \\
$s_{a g g}$ & $2.27-2.68$ & $1.29-2.42$ & $0.20-1.76$ \\
$t \theta_{\text {agg }}$ & $0.72-2.63$ & $0.11-0.61$ & $0.01-0.05$
\end{tabular}

Table 8: A summary of the model values across parameter combinations for aggregate measures of interest (see Table 7 for an explanation of group measures and Appendix A for full data). The range of average maximum values across all 16 parameter combinations ( range $_{\max }$ ), the range of mean values across parameter combinations ( range $_{a v r}$ ), and the range of average minimum values rang $e_{\min }$ give a sense of the behaviour of the model across the 16 parameter combinations being considered. Note that the minimum value for some parameter combinations is higher than the maximum value for other combinations.

Group volume Assuming the minimum size for an adult D. rerio is approximately 2.50 $\mathrm{cm} \times 0.50 \mathrm{~cm} \times 1.00 \mathrm{~cm}$, the minimum volume possible in an actual D. rerio aggregate is approximately $1.25 \mathrm{~cm}^{3} / \mathrm{D}$. rerio, or $22.50 \mathrm{~cm}^{3} / 18 \mathrm{D}$. rerio. In parameter combinations where the spacing urge was less than or equal to the centering urge, and where velocity matching urge was low, the maximum average volume of the group remained below the minimum possible volume, indicating that the model was not behaving in a physically plausible manner. However, this was only the case for 4 of the 16 parameter sets. For the remaining 12 sets the maximum average group volume was sufficiently high to be physically possible. These 12 sets were used when predicting aggregate behaviour of the actual $D$. rerio aggregate.

The variability of simple volume across parameter combinations made it difficult to predict maximum and minimum group size. However, for all parameter combinations maximum and minimum group size varied over time, with the minimum size being anywhere between $4.57-$ $46.89 \%$ smaller than the maximum size. A review of individual runs of the model showed that the simple volume of the group also tended to oscillate over time, with the group expanding or contracting from one moment to the next, rather than steadily increasing or decreasing in value (see Figure 22a). For some parameter combinations there was also a broad trend in density over 
time, with density values increasing or decreasing over time when oscillations were averaged out, but this was not the case with all parameter combinations. Based on this, it would be predicted that the density of the actual D. rerio aggregate would fluctuate over time, increasing and decreasing, but the size of the fluctuations would generally be small.

Group speed Across parameter combinations, the agent group moved at maximum speeds $\left(2.27-2.68 \mathrm{~cm} / 30^{\text {th }}\right.$ second) that were just below the maximum speed of individuals in the group (2.73 $\mathrm{cm} / 30^{\text {th }}$ of a second). Minimum speeds across parameter combinations were more variable $\left(0.20-1.76 \mathrm{~cm} / 30^{\text {th }}\right.$ of a second) but $75 \%$ of the time the difference between maximum and minimum speed was above $1.50 \mathrm{~cm} / 30^{\text {th }}$ second. Average speeds of the aggregate across parameter combinations were $1.29-2.42 \mathrm{~cm} / 30^{\text {th }}$ of a second. Graphs of group movement speed for individual runs of the model (see Figure 22) show that group speed did not slowly accelerate over time, but rather that the group typically increased and then decreased its speed repeatedly throughout the simulation.

Thus it would be predicted, based on this, that the actual D. rerio aggregate would travel at a maximum speed that was $87 \%$ of the maximum speed of the individuals in the group and the aggregate would also have a mean speed close to the maximum speed of individuals in the group. It would further be predicted that there would be a change in this speed over time, with the group slowing down, then speeding up, then slowing down again, and repeating this over the course of the observation period (see Figure 22b).

Group angle With respect to the angle of the group movement over time, the minimum angle across parameter combinations was low (0.0051-0.050 rad), indicating that the group continued in one direction over some timesteps in all parameter combinations. Maximum angle across groups was fairly variable $(0.72-2.63 \mathrm{rad}$, where the maximum possible value was $3.14 \mathrm{rad})$. Graphs of the group angle for individual runs of the model show that the group tended to change direction frequently but that the size of the change was generally small, with the occasional large change in direction indicated by a spike in the angle graph (see Figure 22c). 

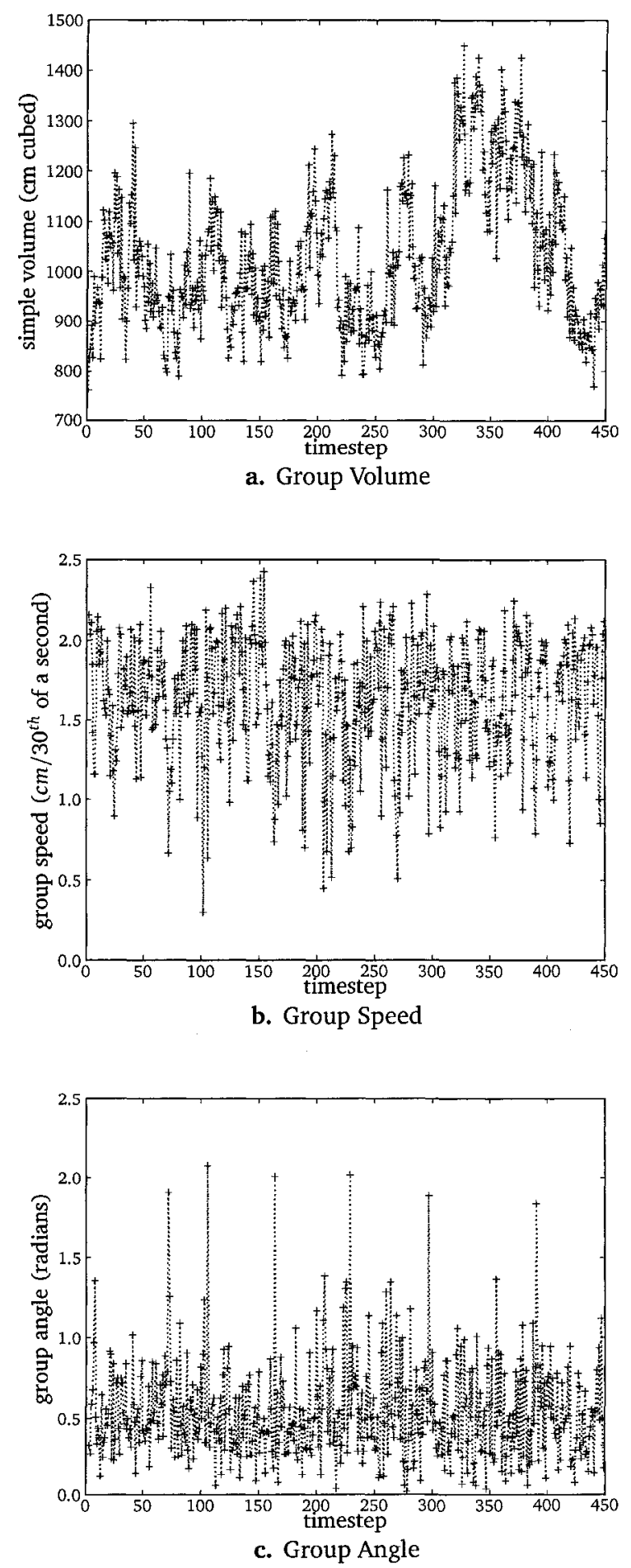

Figure 22: Aggregate volume, speed and angle during a single run of the PCA $D$. rerio model for parameter combination $(10,1,3,3)$. 
Based on this it would be predicted that while at times the actual D. rerio aggregate might briefly move in a straight line, it would also frequently change direction. However, the majority of direction changes would not be expected to be large, with abrupt changes of direction possible, but rarely occurring.

Polarisation Polarisation could not be compared with actual D. rerio aggregate behaviour, because Tracker $3 \mathrm{D}$ analysis does not provide orientation data for fish. However polarisation results and predictions will be presented here in the interest of possible future work. Polarisation was variable across parameter combinations. Maximum polarisation values ranged, across parameter combinations, between 0.39 and 1.50 , with a variety of intermediate values (maximum possible value is 3.14 , indicating the highest possible lack of alignment in the aggregate). Minimum polarisation values ranged between $0.11-0.46$, also with a variety of intermediate values. Even the highest value, 1.49, was quite a bit below the maximum possible value for this measure (3.14), indicating that the group was never highly disordered, and the lowest value, 0.11 indicated strong alignment.

Based on this it would be predicted that while alignment in the actual $D$. rerio aggregate would change over time, the group would always retain a relatively high degree of alignment, and be highly polarised at times. A review of individual runs of the model further support this prediction. Values tend to oscillate but remain low over the course of the run, with the exception of a few spikes of high values (indicating low group alignment) over the course of the run. This further predicts that the aggregate would, for the most part be polarised, with infrequent bursts of unpolarised behaviour.

\subsection{Comparison of PCA model predictions with actual aggregate behaviour and behaviour of simple AR model}

This part of the chapter will provide a comparison of the predictions made by the PCA model with actual behaviour of the aggregate of $18 \mathrm{D}$. rerio. The method used to analyse the behaviour of the group of $18 \mathrm{D}$. rerios will be described in Section 4.5.1. After comparing the model 
predictions to actual $D$. rerio behaviour, predictions made by a classic AR model (composed of zero mass point agents, each with a simple information field) will also be compared with the behaviour of the actual aggregation.

\subsubsection{Collection and analysis of aggregate data}

Group aggregate behaviour data for the $D$. rerio aggregate of 18 fish was collected just after collection of the individual and small group $D$. rerio behavioural data, using the same group of D. rerio. However, data from the group of 18 was not analysed in advance of model creation and analysis. This was done in order to clearly avoid any possibility of modifying the behaviour of the model, a priori, to match the group-level behaviour of the $D$. rerio aggregate. Rather, the model was used to generate predictions about the behaviour of the aggregate, the behaviour of the $D$. rerio aggregate was then analysed and, finally, the model predictions were compared with the behaviour of the actual aggregate.

\section{Methodology}

To collect aggregate behaviour, all 18 fish were placed in the experimental tank and videotaped for 20 minutes, resulting in 36000 frames of data. As with the individual data, data from the first 5 minutes of this trial was discarded prior to analysis. Since these data were intended to validate model predictions of group properties, three measurable group properties were chosen for comparison: group speed, which is the change in group position over time; group movement angle, which is the angle between successive group velocity vectors; and simple group volume (volume measured using a bounding box).

Both group speed and and group angle measurement required, first, a calculation of group position, which in turn required determination of the position of individual fish for a given video frame. It was recognised that, due to limitations associated with the processing software and lab setup, it would be difficult to get accurate position and tracking data for all 18 fish across multiple frames. However, it was generally possible to get position data for some of the 18 fish in each frame. This position data could then be used to provide an estimate of the group position 
of the aggregate in that frame, which in turn could be used to calculate aggregate speed and aggregate angle of movement.

Visual inspection of the video showed that fish in the tank did not remain stationary, and that the centre of the group shifted over time. Thus, both measures where the speed of the aggregate was physically improbable (faster than the highest speed measured for an individual), and measures where the speed of the aggregate was close to zero were suspect. Similarly, volume measures where the volume was lower than $22.50 \mathrm{~cm}^{3}$, the approximate minimum calculated volume for $18 \mathrm{D}$. rerio, were also suspect. Two approaches were taken to deal with this erroneous data. The first was to take an overall mean across all values calculated for two or more fish, under the assumption that high and low errors occurred with similar regularity and would balance each other out, thus resulting in a reasonable estimate of the actual mean. The second strategy was to eliminate all values above or below certain threshold values. For calculating group speed, values greater than $3.00 \mathrm{~cm} / 30^{\text {th }}$ of a second (with reference to the maximum measured speed of an individual, $2.73 \mathrm{~cm} / 30^{\text {th }}$ of a second) were eliminated. For calculating angles, angles were only calculated if the speed vectors associated with the angle were less than $3.00 \mathrm{~cm} / 30^{\text {th }}$ of a second. For group volume, values below $22.50 \mathrm{~cm}^{3}$ were eliminated.

\section{Results}

A summary of the results of the data analysis are presented in Table 9. These results are discussed below.

\begin{tabular}{lll} 
measure & all data points & cleaned data \\
\hline$v_{\text {simp }}\left(\mathrm{cm}^{3}\right)$ & $2774.77 \pm 4516.45(\mathrm{n}=4058)$ & $2984.02 \pm 4617.35(\mathrm{n}=4392)$ \\
$s_{\text {agg }}\left(\mathrm{cm} / 30^{t h} \mathrm{sec}.\right)$ & $2.60 \pm 4.06(\mathrm{n}=4365)$ & $0.66 \pm 0.76(\mathrm{n}=3122)$ \\
$t \theta_{\text {agg }}(\mathrm{r})$ & $1.53 \pm 1.07(\mathrm{n}=4337)$ & $1.25 \pm 0.96(\mathrm{n}=2096)$
\end{tabular}

Table 9: Group measures of the behaviour of 18 D. rerio: In the cleaned data, speeds above $3.00 \mathrm{~cm} / 30^{\text {th }}$ of a second were removed, angles calculated using speeds above this were removed and densities < 22.50 or $>202475.00 \mathrm{~cm}^{3}$ were removed (but no measurement was above the maximum possible density value). 


\subsubsection{Comparison of aggregate behaviour with model predictions}

Speed The mean speed of the aggregate as predicted by the model was $1.29-2.42 \mathrm{~cm} / 30^{\text {th }}$ of a second. If values above $3.00 / 30^{\text {th }}$ of a second were eliminated then the mean speed of the actual D. rerio aggregate was recorded as $0.66 \pm 0.76 / 30^{\text {th }}$ of a second and if all values were included the mean speed of the actual D. rerio aggregate was recorded as $2.60 \pm 4.06 \mathrm{~cm} / 30^{\text {th }}$ (the difference between these values was caused by the large number of values, 1243, that were above 3.00 , with a mean value of 7.45). Thus, the actual mean speed of the aggregate is below the range predicted by the model when using cleaned data, and above the range predicted by the model when using all data(a significant difference, $p<0.05$, for both cleaned data and all data points compared with values at each end of the model range). From a qualitative point of view, group speed behaviour corresponded to the behaviour predicted by the model, with the speed oscillating over time (see Figure 23b).

Group angle Qualitatively, the model predicted that the turning angle of the group would be generally low, with occasional jumps in value. Quantitatively, the average change of direction predicted by the model was $0.11-0.61$ with maximums of $0.72-2.63$. Observed average change of direction (cleaned data) was $1.25 \pm 0.96$. Thus the actual value was significantly higher ( $p<$ 0.005 ) than the predicted mean value, but within the range of the maximum values predicted. With respect to the pattern of movement, actual group angle data showed the group frequently changing direction over time, with some spikes in turning angle as predicted by the model. (see Figure 23c).

Group volume The mean value predicted for density across parameter combinations was $1049.62 \mathrm{~cm}^{3}$, and the pattern of the volume change over time predicted by the model, was that the simple volume would increase and decrease over time, rather than remaining constant or increasing continuously. The mean simple volume actually recorded was $2984.02 \pm 4617.35 \mathrm{~cm}^{3}$, which was significantly higher $(p<0.005)$ than the predicted maximum volume. However, like the model, the simple volume of the aggregate increased and decreased over time (see Figure 23a). 


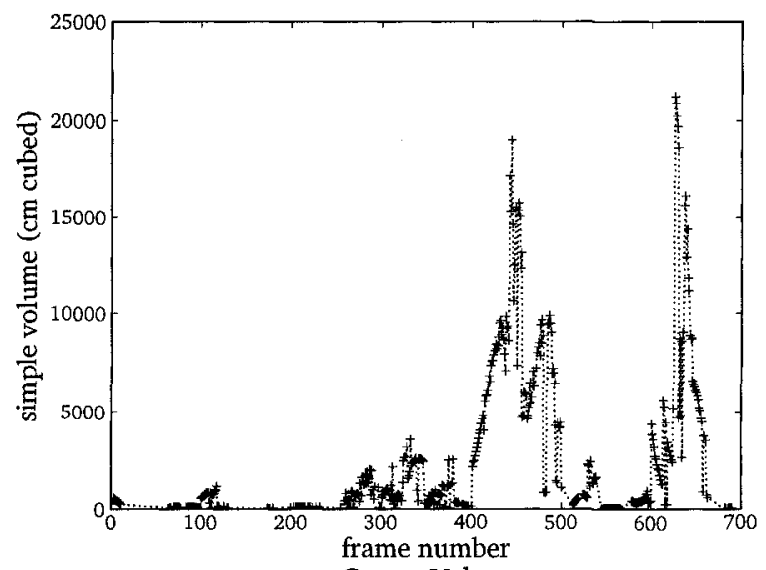

a. Group Volume

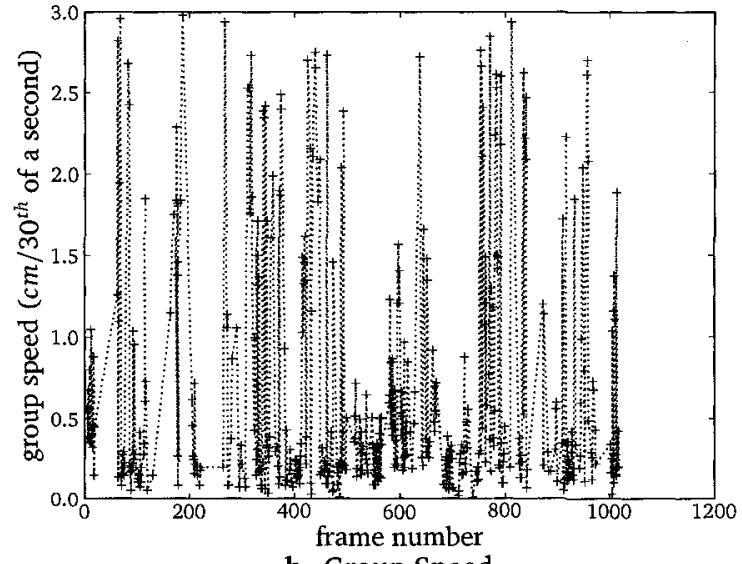

b. Group Speed

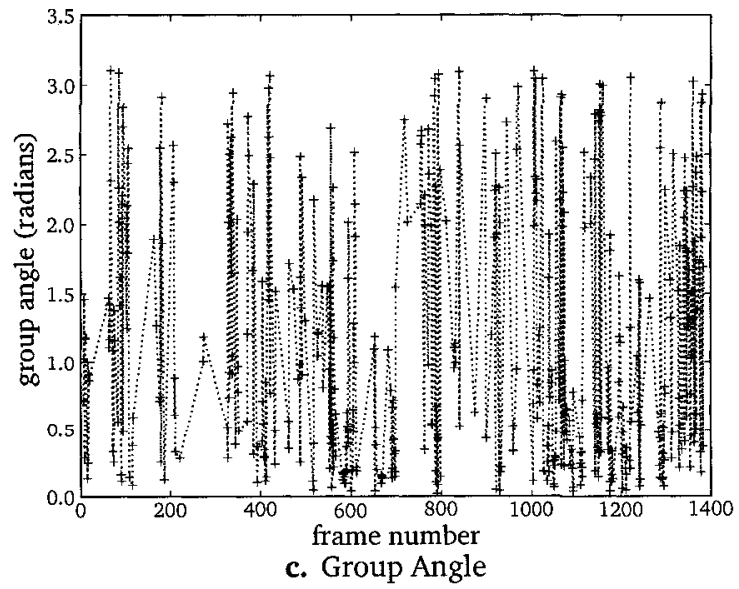

Figure 23: Aggregate volume, speed and angle for a $D$. rerio aggregate of $18 \mathrm{D}$. rerio. 


\subsubsection{Comparison of aggregate behaviour with behaviour of simple AR model}

The behaviour of the $D$. rerio aggregate was also compared with the behaviour of a simple AR model, to see if the simple AR model could make accurate predictions about the behaviour of the actual aggregate when parameters of the AR model were set using the experimentally collected values for maximum speed, turning angle and preferred spacing. This was done to show that models made using the new PCA modelling framework could provide information and predictions that previous models would not have made, and also to show the importance of incorporating experimental data into models in order to validate them - something which is made more feasible through use of the PCA modelling framework.

\section{Model setup and methodology}

The simple AR $D$. rerio model was created by altering the code of the PCAI $D$. rerio model discussed in Section 4.3. Algorithms 1 and 2 still describe the model. However, in the simple attraction and repulsion model, agent embodiment was removed so that agents were represented as zero volume points, rather than the 5 sphere agent body in the PCAI model. Agents had a spherical perceptual area of 40 units, rather than the 8 cone-shaped perceptual fields of the PCAI model, so agents detected all other agents with a 40 unit radius. Each parameter combination was run 35 times, for 501 timesteps each, with agents initially positioned at $(0,0,0)$. Model movement and preferred distance parameters were set using the experimentally gathered data, and the same cognitive algorithms were used. The same parameter combinations were run and analysed in the same way.

\section{Results}

A visual inspection of the model behaviour showed evidence of aggregate behaviour for some parameter combinations, with agents moving together in a cohesive group through space. However, for all but four of the parameter combinations, the simple AR model had densities that were far below the minimum possible D. rerio densities $\left(>0.033 \mathrm{~cm}^{3} / \mathrm{D}\right.$. rerio, where the minimum density for actual aggregates is approximately $1.25 \mathrm{~cm}^{3} / D$. rerio). The remaining four 
models remained essentially stationary in space, as indicated by low group speed (an average maximum value across parameter combinations of $0.11 \mathrm{~cm} / 30^{\text {th }}$ second) across timesteps. A visual inspection of these models showed agents spread out from each other and spinning around their own axes, rather than moving as a group.

With respect to polarisation and angle of movement, parameter combinations that resulted in group movement (albeit with physically impossible density) showed polarisation and angle movement values that were somewhat similar to the group of 18 and PCA model in terms of range of values. Average group angles were between 0.13 and $1.44 \mathrm{rad}$, as compared with the observed mean change of direction (cleaned data) of $1.25 \pm 0.96$ rad. With respect to minimum values, parameter combinations generally oscillated between 0.005 and $0.99 \mathrm{rad}$, with most of the parameters settings (12 of the 16) having a low minimum value.

With respect to polarisation, values could not be compared with the actual aggregate. Maximum values ranged between 0.29-1.59 rad and minimum values ranged, similarly, between $0.12-1.04 \mathrm{rad}$. Average values were between $0.22-1.37 \mathrm{rad}$. For some parameter combinations the model predicted almost total alignment at all times, while for other parameter combinations the model predicted quite low alignment in general. This variability would make it difficult to predict actual aggregate behaviour. As well, the AR model exhibited evidence of attraction to particular model states, with different parameter combinations producing the same qualitative and quantitative model behaviours.

Aggregate volume, speed and turning angle graphs for a single run (see Figure 24) demonstrate that the qualitative behaviour of the model for these aggregate measures was noticeably distinct from the qualitative behaviour of the actual aggregate, particularly with respect to group volume (see Figure 23). In the graphed AR run, group volume steadily increased and then plateaued, rather than oscillating between values, with occasional spikes in volume (the behaviour seen in the graph of the actual aggregate volume). Group speed and group angle for the AR model exhibited some degree of oscillation. However this was also in the context of a larger trend, with group speed decreasing and then plateauing and group angle increasing and plateauing, accompanied by an increase in size of oscillations. This was in contrast to the actual group behaviour, which showed consistently oscillating behaviour throughout the trial period. 


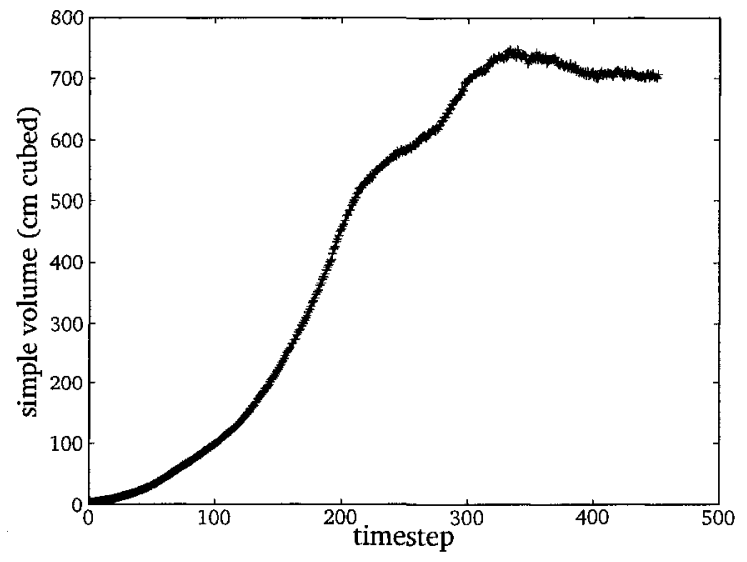

a. Group Volume

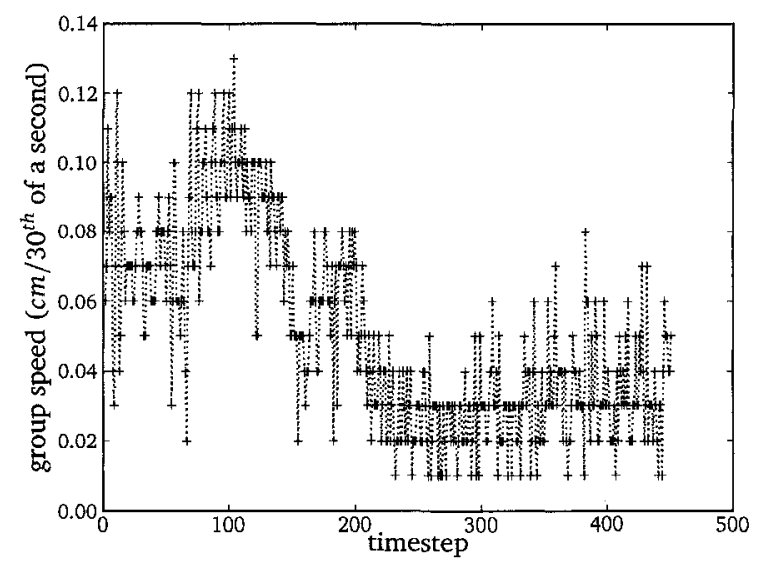

b. Group Speed

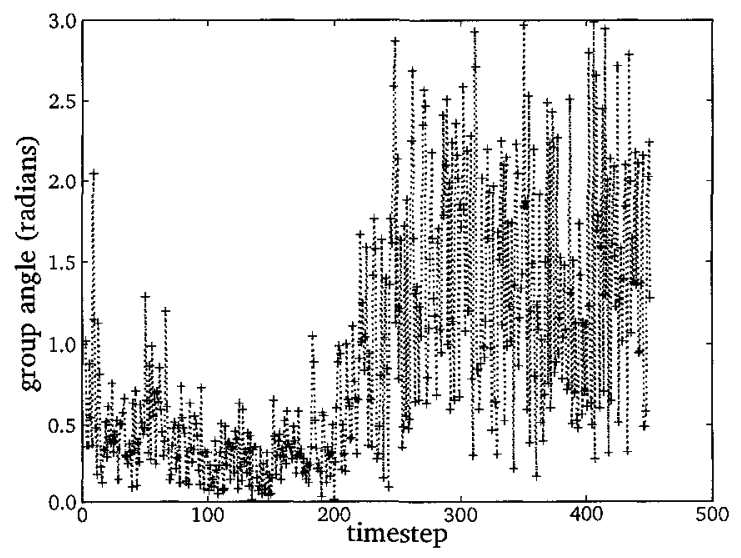

c. Group Angle

Figure 24: Aggregate volume, speed and angle during a single run of the simple $D$. rerio model for parameter combination $(10,1,3,3)$. 


\subsection{Discussion}

Previously, modellers that have based the behaviour of their modelled individuals on experimental data have rarely tried to validate their models' behaviour by comparing the aggregate behaviour of the model with the behaviour of an actual aggregate. However, as has been shown by the results presented in this chapter, it is possible to do so. Qualitatively, the PCAI $D$. rerio model predicted that simple volume, aggregate speed and aggregate angle values would oscillate over time. This behaviour was also seen in the actual aggregate for all of these measures.

With respect to quantitative predictions, values predicted by the model were generally significantly different from values seen in the actual aggregate. However, the large sample size (and resulting statistical power) meant that even relatively small differences could be significant, and the magnitude of the difference between the model's predictions and the actual values were not always that large. For example, the value of the aggregate volume predicted by the model was smaller than the value seen in the actual model by an amount roughly comparable to the size of two decks of cards. In all cases the value predicted by the model was within the same order of magnitude as the measured behaviour of the aggregate.

In contrast to this, the simple AR model failed to generate realistic qualitative and quantitative predictions. Although its group angle values were closer to those seen in the actual aggregate, this only occurred for parameter combinations where the density of the aggregate was impossibly high. When considered across all parameter combinations, the simple AR D. rerio model was an extremely poor predictor of the observed aggregate behaviour, despite having its parameters set using the same data. Specifically, when set using experimentally based parameters, and eliminating parameter combinations that were not physically possible, the simple AR model entirely failed to make realistic qualitative predictions about the behaviour of the $D$. rerio aggregate with respect to aggregate volume and speed. Consequently, although the AR model may be able to produce results that are of interest when parameters are set using non experimental values, it was entirely unsuccessful at creating a predictive model of a species-specific aggregate. 
One of the challenges of using the PCAI model to make predictions was the fact that values for all model parameters were not known. This was particularly the case for cognitive parameters. As a result, model behaviour had to be explored for multiple values of these parameters in order to determine common model behaviours. As well, model behaviour was sensitive to these changes in parameters, and, as indicated in Table 8 , these had a noticeable effect on the range of values seen in the model. This increased the difficulty of using the model to make predictions. Nonetheless, it was still possible to make predictions by finding commonalities among model behaviours under different parameter settings. This was arguably a more cautious approach than simply picking one model parameter setting and using it as the appropriate model parameter setting.

The results of the AR model support the idea that incorporating realistic representations of perceptions in aggregate models is important in order to obtain realistic model behaviour. If these perceptions are not included, then even experimentally set parameters may not result in realistic aggregate behaviour. However, since both multiple perceptual fields and embodied agents were introduced at the same time it is possible that the failure of the simple model was due to the point mass or the simple field, or both. Having said this, it is arguable that to accurately model perceptual abilities, agents also need to be embodied accurately, because perceptions interact directly with objects, resulting in an interaction between these two aspects of the model.

With respect to how these results reflect on existing models, it can be argued that the simple AR model was more simplistic than existing AR models, like those produced by Hemelrijk and Hildenbrandt (2007) and Viscido et al. (2007), because it lacked specific zones in which its rules were applied, and it did not incorporate blind spots into its perceptual field. It also made no effort to embody agents realistically. However, its results are relevant in that they show that even if a model produces qualitative behaviour that is convincing for some parameter settings, its quantitative results may not be realistic when compared with the behaviour of an actual aggregate of interest, even if parameters are set using realistic values. These results also suggest that using generic or non-species-specific models to generate predictions about 
species-specific aggregate behaviour may lead to incorrect predictions, even if, again, available model parameters are set using species-specific data.

This chapter has been, in part, a response to Parrish and Edelstein-Keshet's (1999) concern (see Chapter 3) that even if a model exhibits aggregate behaviour, this does not in and of itself imply that the model is a model of a particular aggregate of interest. The reason for this is that multiple mechanisms can generate aggregate behaviour and there is no guarantee that the mechanisms in the model are the same ones that are being used by the aggregate in question.

As stated in Chapter 3, the way to overcome this problem is twofold. First, the modeller must match the parts of the model as much as possible to the parts of the system actually being modelled, determining which parts of the system are relevant to the phenomenon of interest, and which parts are extraneous. Then, second, the model must be validated, to show that it has included and accurately modelled the parts of the system that are required for the system as a whole to behave in the manner relevant to the system being studied. In Chapter 3 it was argued that the relevant parts of an aggregate include the perceptual, cognitive and movement abilities of the aggregating animals. To constrain a model such that it is a convincing model of a particular species, these components of the model must be clearly matched with the behaviour of the aggregating individuals in question. Once this has occurred, the model may be validated by comparing it with the behaviour of the actual aggregate of interest.

In this chapter, these modelling requirements were carried out explicitly, with perceptual, cognitive and movement properties of individuals in the $D$. rerio model constructed based on empirical data gathered from individuals. Although it was still possible to represent these aspects of individuals inadequately or incorrectly in the model, the model was explicit about the extent to which these aspects of the model did or did not match the properties of actual D. rerio, allowing the issue of model accuracy to be addressed directly. For example, because parts of the cognitive algorithms were not based on experimental evidence, it was possible that the agents in the model were not aggregating in the same manner that actual $D$. rerio were. However, by making the representation and assumptions concerning cognition explicit, this became amenable to explicit validation. 


\subsection{Chapter summary}

This chapter presented the PCAI, a three-dimensional implementation of the PCA framework in the Breve simulation environment. The PCAI was then used to create a species-specific $D$. rerio model. The major findings of this chapter were that it was possible to create a speciesspecific aggregate model that made successful qualitative and, to some degree, quantitative predictions about the behaviour of an actual $D$. rerio aggregate. By contrast, the simple AR D. rerio aggregate model was invalidated as a species-specific model. This supported the contention that embodied agents with accurately modelled perceptions, cognition and action are required if models are to successfully depict and explain aggregate behaviours. The next chapter, Chapter 5 , will investigate the influence that the shape of the perceptual field has on the behaviour of the aggregate, considering in the process the information requirements for cohesive aggregation. Chapter 5 will also examine the ways in which the shape of the perceptual field helps to determine the behaviour of the aggregate and why certain perceptual field shapes are more conducive to aggregate cohesion. 


\section{Chapter 5}

\section{Perceptual underpinnings of aggregate behaviour}

Chapter 4 demonstrated that species-specific, predictive models with physically embodied agents and parameters based on experimental data can be made using the PCA framework. Chapter 5 will extend the findings of Chapter 4 by using the PCA framework to explore, more generally, the information requirements for cohesive aggregate behaviour and the ways in which these information requirements are mediated by the perceptual abilities of aggregating animals. Specifically, Chapter 5 will report the results of model scenarios that show that it is the type of information available, rather than the quantity of information available, that determines whether aggregate cohesion is possible. It will further be shown that the type of information available to individuals is, in turn, mediated by the range of their perceptions, as represented in the model by the shape of the agent's perceptual field.

To support these findings, first, an information-specific definition of aggregation will be presented. Then results relating to the minimum amount and type of information required for aggregation will be presented. Finally, the majority of Chapter 5 will focus on presenting model scenarios that demonstrate how the shape of the perceptual field influences the type of information available to the agent, and how this, in turn influences the cohesion of the aggregate. 


\subsection{An information based definition of aggregation}

Chapter 3 argued that aggregate behaviour can be more successfully understood by using an information processing perspective when modelling individual behaviour. In this context, the aggregate as a whole may also be defined with respect to the information possessed by each agent in the aggregate and that agent's ability to respond to this information. The broadest possible definition would be to define an aggregate as a group of two or more animals, each of which, at a given point in time, $t$, has spatial information about at least one other animal of the same species, which, itself, also has information about at least one other animal of the same species and each of which are capable of adjusting their spatial properties using this information.

This may be more precisely defined, in graph theory terms, if agents are considered to be vertices in a directed graph, in which edges represent an agent having spatial information about another agent. In this case, as long as this aggregate information graph is connected (i.e., every pair of vertices is connected by a path) over time, then the group of agents so connected may be considered to be an aggregate, in a broad sense (see Figure 25). In this broadest definition, coordinated movement may not be present at time $t$ but animals are still defined as an aggregate based on possibility of coordinated movement at time $t$.

With respect to connections over time, and the ability of agents to leave or join the aggregate, the general definition above may be made more precise by saying that, so long as no vertex, $v$, of the graph remains disconnected over a specified period of time, $\delta t_{v}$, then that vertex (and by extension the agent) can be considered to remain within the aggregate. Theoretically, the value of $\delta t_{v}$ can be further defined as the length of time after which $v$ can never again be, or simply is never again, connected to any other vertices. In this latter case, $\delta t_{v}$ would be an a posteriori value. In the former case, it may be possible to determine the value theoretically.For particular aggregates there may also be a typical period of time, $\delta t_{k}$, after which it is improbable that a vertex that is disconnected to the aggregate will be reconnected to the aggregate.

Other definitions of aggregation- ones based on aggregate density, for example- may be viewed as secondary definitions that try to operationalise this information processing definition using measurable properties like distance between animals or number of animals in a particular 


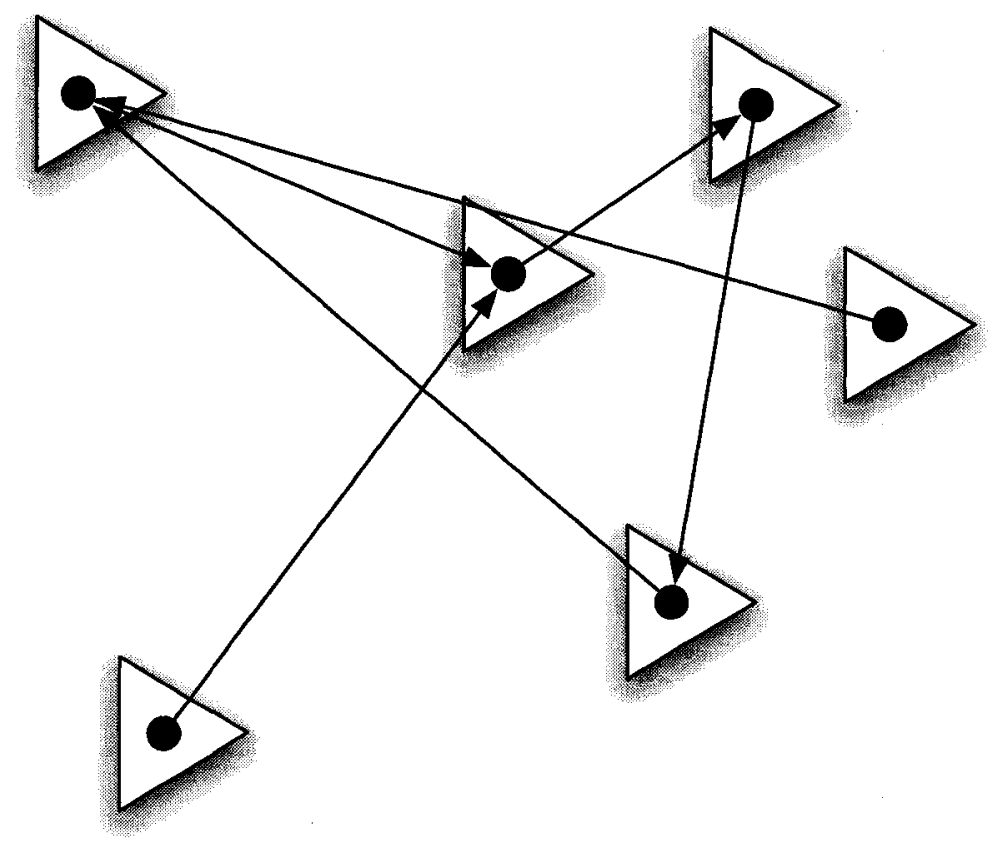

Figure 25: Animal aggregation, as represented by an aggregate information graph showing which animal has information about which other animals. Lines represent information, with the start of the arrow indicating the animal that has the information and the direction of the arrow indicating which animal the first has information about. 
area or volume of space. These operationalisations of the information processing definition are necessary from an experimental point of view because it is not possible to observe the information one animal has about another. However, these operationalisations are only valid if it is assumed that there is a relationship between, for example, the distance between one animal and another and the ability of the first animal to obtain information about the other animal. This assumption seems reasonable if the concept of the perceptual field is added as a mediating factor. It is also worth noting that in some cases (e.g. whales communicating with ultrasound (Frazer and Mercado III, 2000), humans communicating with cell phones), some forms of aggregate behaviour may be possible over large areas, and at very low densities of the animal in question. Thus, efforts to operationalise the definition of an aggregate using measures like density and volume of aggregate need to be species and environment specific. An operationalised definition of aggregation is used to measure aggregate cohesion in the information and perceptual field investigations discussed below. The results are discussed both within this context and in terms of the primary aggregate information graph definition of aggregation.

Continuing to discuss aggregation with respect to information processing, and in terms of graphs, it may be asked: How much information is required, or, in graph terms, how connected does the aggregate information graph need to be, to generate physically measurable aggregate behaviour (i.e., measurable using an operationalised definition of an aggregate)? It may also be asked- is it the number of connections or the type of connections which are most important in determining the existence of aggregate behaviour? In the rest of this chapter the results of models created to answer these questions will be discussed, particularly with respect to how perceptual abilities mediate the ability of aggregates to acquire sufficient information to form aggregates.

\subsection{Four scenarios exploring the minimum information required for aggregation}

To explore how information limitations might affect the behaviour of an aggregate, four scenarios, all varying the amount and type of information available to agents, were compared. In the first 
two scenarios, every agent in the simulation always had access to at least some information about other agents, but the amount and type of information was varied. In scenarios three and four, the first two scenarios were repeated, with the exception that some agents did not receive any information about other agents at each timestep. Lastly, a control scenario was also run where agents had access to and used all information about other agents at each timestep.

\subsubsection{Model setup and methodology}

Agent information The models for the minimum information scenarios were based on the D. rerio model constructed in Chapter 4 (see Algorithms 1 and 2). At this stage, agent perception was not modelled, in order to allow for a direct investigation of theoretical information constraints (see Section 5.3 for a discussion of models that extend these findings to models with perceptual fields). Instead, to eliminate variables in the preliminary investigation, environmental information was provided directly to the attention function, with attention algorithms as described in Algorithm 3. Thirty agents were used for each scenario. In the first scenariothe random agents scenario- agents randomly picked a specified number, $N$, of other agents (referred to as their neighbours) at each time step, and used information about these agents as input to their cognitive algorithms. In the second scenario- the nearest neighbour scenarioagents still picked a specified number of the agents, but in this case they picked only the $N$ agents nearest to them (referred to as their $N$ nearest neighbours) and used information about these agents as input to their cognitive algorithms (see Algorithm 3 for algorithm details). The third scenario was the same as the first scenario and the fourth the same as the second, but with a specified number of randomly chosen agents at each turn (the perception deprived agents, $\Phi$ ) receiving no information for that turn.

For both the first and second scenarios, the number of neighbours picked varied from 1 to $16(1,4,8,12$ and 16 neighbours, where 16 is just over half of the aggregate). There was also a control run where agents used information about all other agents in the group. In the final two scenarios (where some agents in each timestep received no information about the aggregate) 


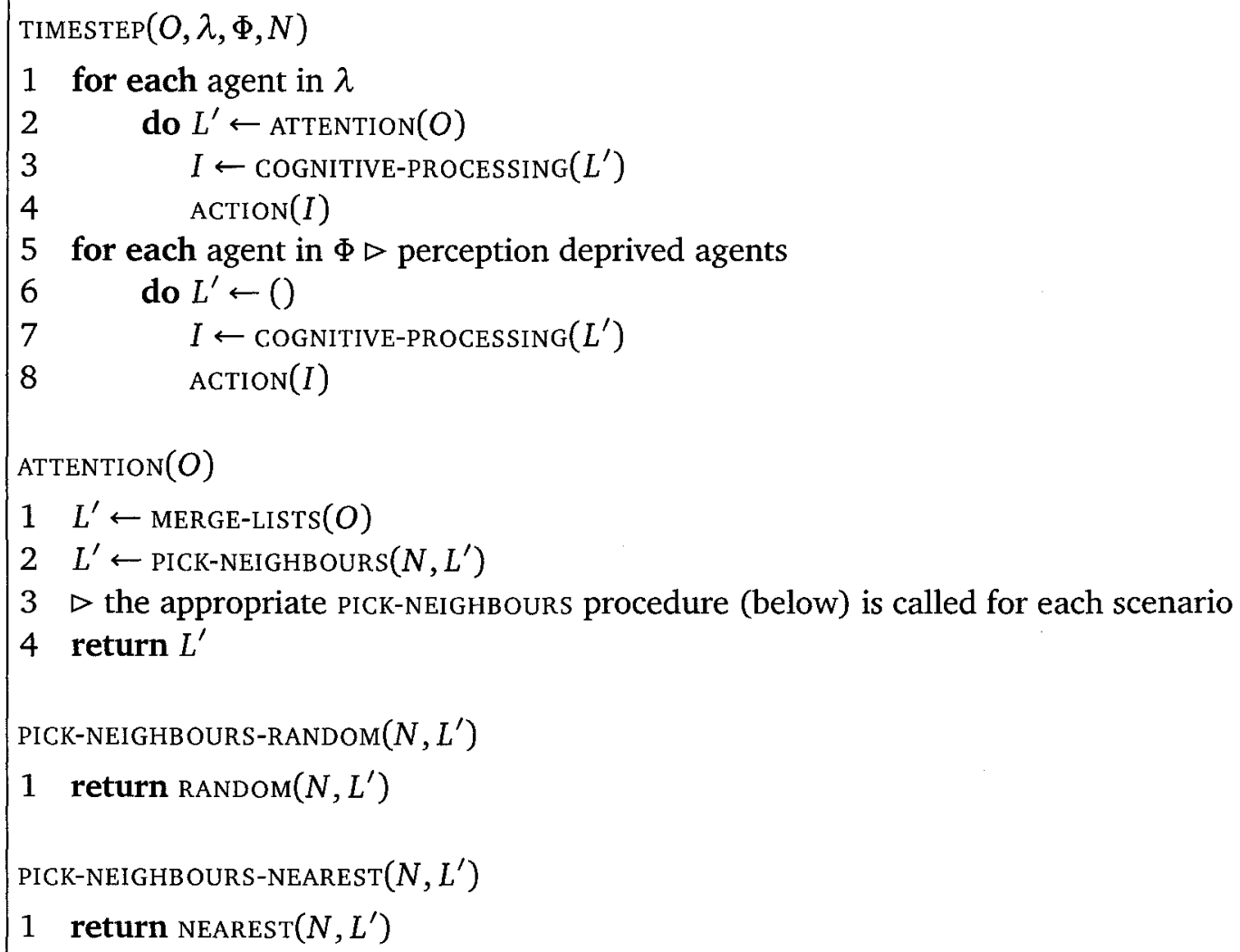

Algorithm 3: Agent behaviour during one timestep of the minimum information models. Cognitive processing and action algorithms are the same as in the Chapter $4 \mathrm{D}$. rerio model, Algorithm 2.

models were run where $1,4,8,12$ and 15 (half of the group) randomly selected agents at each time step received no information.

Parameter combinations Within the context of these four main scenarios a number of additional parameter combinations were used to explore behaviour across the parameter space of the scenarios. Specifically, two values for maximum speed, maximum turning angle and preferred space were chosen and two different starting configurations were chosen, one where all agents started with a random position within one unit radius of the origin, and one where all agents started with a random position within a 40 unit radius of the origin (see Table 10). This resulted in a total of of 80 parameter combinations for each of the first two scenarios. 
Model runs and aggregate measures Model runs of 100 timesteps each were run for each parameter combination, with 10 model runs per parameter combination. At each timestep, information about the number of stragglers, groups and clusters was gathered, where, as in Chapter 4, stragglers were defined as agents not within 20 units of any other agent, groups were sets of agents that were within 20 units of at least one other agent already in the group, and a cluster was either a group or a straggler. Each of these measures provides a different type of information about the aggregate: number of stragglers provides information about how many individuals are effectively 'lost', number of groups provides information about whether or not the main aggregate has split into (possibly stable) smaller aggregates, and clusters provides an overall measure of group cohesiveness, from the perspective that either stragglers or subgroups that have separated from the main group represent a reduction of cohesion. Maximum number of clusters, groups and stragglers for each run were calculated and averaged across runs and these collected results were further compared and analysed across parameter combinations to determine how parameter settings influenced these aggregate measures. Differences between values were confirmed using either paired t-tests (for comparisons of two values) or one-way repeated measures ANOVAs followed by a Tukey HSD test for multiple comparisons. Correlation was determined using Pearson's correlation coefficient.

\begin{tabular}{ll} 
Parameters & Values \\
\hline$s_{\max }$ & $0.5 \mathrm{u} / \mathrm{t}, 2.0 \mathrm{u} / \mathrm{t}$ \\
$t \theta_{\max }$ & $0.78 \mathrm{rad}, 3.10 \mathrm{rad}$ \\
space $_{\text {pref }}$ & $2 \mathrm{u}, 10 \mathrm{u}$ \\
$w_{c}$ & 1 \\
$w_{v}$ & 1 \\
$w_{s}$ & 3 \\
starting position radius $\left(r_{s p}\right)$ & $1 \mathrm{u}, 40 \mathrm{u}$ \\
number of neighbours perceived $\left(n b s_{\text {perceived }}\right)$ & $1,4,8,12,16$ \\
number of perception deprived $(p d)$ agents & $1,4,8,12,15$
\end{tabular}

Table 10: Parameter values for the four information scenarios: Number of perception deprived (pd) agents are the number of agents in a timestep that do not receive any information in Scenarios 3 and 4 $(\mathrm{u}=$ units of distance, $\mathrm{t}=$ timestep). 


\subsubsection{Results}

Results when all agents perceived As might be expected, in the control scenario, when agents used all information about the aggregate to determine movement, the aggregate remained as a single group for almost all runs of all parameter sets. Only 1 straggler occurred across all 160 model runs of 100 timesteps each.

For the random neighbour scenario, the aggregate remained fully cohesive (the aggregate remained as a single cluster across all 10 runs of the parameter combination) for 58 out of 80 parameter combinations. For each of the remaining 22 parameter combinations, the aggregate remained as a single cluster for at least $8 / 10$ runs. Combining parameter combinations in which the same number of neighbours determined movement, the number of neighbours was not significantly correlated with number of parameter combinations where number of clusters was greater than 1, or with total number of clusters within each group (see Table 11, $n=5, r=$ $0.10, t(3)=0.02 p>0.1, r=-0.08, t(3)=-0.14, p>0.1)$.

\begin{tabular}{lllll} 
& \multicolumn{2}{l}{ random neighbour } & \multicolumn{2}{c}{ nearest neighbour } \\
$n$ ss $_{\text {perceived }}$ & clusters $_{>1}$ & clusters $_{\text {tot }}$ & clusters $_{>1}$ & clusters $_{\text {tot }}$ \\
\hline 1 & 4 & 16.7 & 13 & 70.6 \\
4 & 6 & 16.8 & 11 & 36.2 \\
8 & 3 & 16.4 & 13 & 34.7 \\
12 & 3 & 16.5 & 11 & 32.4 \\
16 & 6 & 16.8 & 13 & 31.5
\end{tabular}

Table 11: Clusters relative to number of neighbours perceived by agents: For parameter combinations with the indicated number of neighbours perceived $\left(n b s_{\text {perceived }}\right)$, this table shows the total number of parameter combinations where the number of clusters was greater than one (clusters $s_{>1}$ ), and the total number of clusters across parameter combinations where agents had information about a number of other agents up to the indicated number of neighbours ( clusters $_{t o t}$ ). Data is shown for both the random neighbour and nearest neighbour scenario.

In the nearest neighbour scenarios, the aggregate consistently broke up into smaller groups for all numbers of neighbours considered (in 61 out of 80 parameter combinations the aggregate lost cohesion during at least one run). There was no significant correlation between the number of neighbours perceived by agents and the number of parameter combinations where clusters were greater than $1(n=5, r=-0.03 t(3)=0.05 p>0.1)$ (see Table 11). The total number of 
groups across parameter settings for a particular number of neighbours was negatively, but not significantly, correlated with the number of neighbours perceived by agents $(n=5, r=-0.74$, $t(3)=-1.9, p>0.1)$. When one nearest neighbour was considered, the mean number of clusters was 4.41. When 16 nearest neighbours were considered, the mean number of clusters was 1.97 . Here, the largest change in value was between perceiving 1 and 4 agents. Perceiving between 4 and 16 agents resulted in a similar number of clusters.

Results when some agents were perception deprived Table 12 allows for a comparison of two variants of the random neighbour scenario. A comparison of model behaviour when each agent selected 8 agents to determine movement and all but one agent had perception each timestep with the same scenario but with 15 randomly chosen agents with no perception for each timestep, showed negligible differences for both the random neighbour algorithm and the nearest neighbour algorithm for number of groups (see Table 12). However, number of stragglers, and consequently overall cohesion, as represented by number of clusters, did differ significantly (although not largely) between the single $p d$ agent case and the $15 p d$ agent case in both the nearest neighbour and the random neighbour scenarios.

\subsubsection{Discussion}

The four scenarios discussed in this section— the random neighbour scenario, the nearest neighbour scenario and these scenarios repeated but with some agents perception deprived- were compared in order to gain an understanding of the minimum information requirements for aggregate cohesion. The results from these scenarios show that an aggregate can maintain cohesion if as few as half of the agents in the group at each moment in time have information about a single other agent. However, this is largely true only if that single agent is randomly chosen from the set of all agents in the simulation. If agents base their movement on information about their nearest neighbours rather than on neighbours randomly chosen, the resulting aggregates are much less reliably cohesive. The relevance of these results to existing research will be discussed below. 


\begin{tabular}{llll} 
scenario & groups & stragglers & clusters \\
\hline \hline nearest neighbour & & & \\
1 pd agent & 45.65 & 1.1 & 45.9 \\
15 pd agents & 45.4 & 2.8 & 47.25 \\
\hline significant difference & no & yes & yes \\
& $(t(15)=0.86$ & $(t(15)=-3.17$ & $(t(15)=-2.30$ \\
& $p=0.40)$ & $p=0.006)$ & $p=0.036)$ \\
\hline \hline random neighbour & & & \\
1 pd agent & 16 & 0.6 & 16.6 \\
15 pd agents & 16.1 & 2.4 & 18.5 \\
\hline significant difference & no & yes & yes \\
& $(t(15)=1.00$ & $(t(15)=2.42$ & $(t(15)=2.60$ \\
& $p=0.33)$ & $p=0.029)$ & $p=0.02)$ \\
\hline \hline
\end{tabular}

Table 12: A comparison of behaviour between model runs where all but one agent perceived eight neighbours at each timestep and model runs where only half of the group perceived eight agents and half of the group perceived no agents at each timestep ( $n=16$, paired t-test).

In aggregate information graph terms, these results show that it is possible for an aggregate of $n$ individuals to maintain cohesion over time if vertices that are disconnected from the aggregate information graph at each timestep are chosen randomly. The number of agents that do not perceive other agents at a single timestep $(p d)$ has a relatively small (albeit statistically significant) influence on this, such that even when $p d=n / 2$ there is only a minimal effect on cohesion, as a result of a small increase in number of stragglers. It is speculated that so long as an agent can maintain a sufficiently accurate representation of the aggregate over time to successfully re-establish a connection to the aggregate information graph after a passage of time, that agent does not need to have information about the aggregate at every moment in time in order to remain with the aggregate. If, however, connections are established in such a way that nodes in the graph are only connected to nearest neighbours, the results show that the aggregate will tend to lose cohesion, even if the size of the information graph is $n$, and vertices have degrees as high as $n / 2$. It is speculated that this is because the nearest neighbour algorithm tends to result in the formation of disjoint subgraphs (to be investigated in future work). 
The results of this section call into question the generality of the conclusions drawn by models using the nearest neighbour algorithm. Parrish et al. (2002), using a nearest neighbour algorithm in their model of fish schooling, conclude that when schooling fish attend to more neighbours, this leads to larger aggregates, because this increases the number of neighbours shared among individuals in the aggregate and the aggregate is able to maintain higher levels of cohesion. However, as has been shown here, this result is specific to the nearest neighbour algorithm, and is not necessarily the case more generally. Instead, it may be possible for animals to aggregate in large groups even when considering only small amounts of information, and attending to more and more information may not improve group cohesiveness.

Viscido et al. (2005) also consider the influence of numbers of neighbours on aggregate behaviour using the nearest neighbour algorithm. They find that group cohesion is reliably maintained only once the number of neighbours considered is approximately half of the size of the entire group. However, again, this is specifically the case for the nearest neighbour algorithm and does not hold true for the random neighbour algorithm. Rather, in the case of the random neighbour algorithm, the cohesion of the group can be maintained by animals using a single piece of information about the aggregate, assuming that this piece of information is chosen in such a way that it maximises information about the behaviour of the group as a whole.

Ballerini, Cabibbo, Candelier, Cavagna, Cisbani, Giardina, Lecomte et al., (2008) suggest that, based on the movement patterns of European starlings (Sturnus vulgaris), they are using what the refer to as 'topological' rather than 'metric' distance when determining their force of attraction to other starlings. In other words, a starling would be equally attracted to two starlingss that were different distances apart, as long as they were the two closest starlings. This is effectively the nearest neighbour algorithm, and Ballerini, Cabibbo, Candelier, Cavagna, Cisbani, Giardina, Lecomte et al., (2008) argue that their data supports the idea that starlings use some form of this algorithm to allow them to aggregate in large groups. However, results from the models discussed here suggest that the nearest neighbour algorithm generally leads to low aggregate cohesion, even in large groups. This contrasting result suggests that there may be alternate interpretations of the starling data. 
The scenarios explored in this section were not meant to be realistic scenarios, but were constructed in order to explore the minimum information requirements for aggregate cohesion. Consequently it might be argued that animals cannot realistically obtain a random sample of information about the agents in their environment, and that the nearest neighbour algorithm is a more plausible representation of actual animal behaviour. Ultimately, however, the type of information that animals can obtain from their environment is mediated by their perceptual fields, and so the realism of these algorithms can only be determined by considering the operation of these perceptual fields. The ways in which the shape of the perceptual field interacts with the information to which an animal chooses to attend will be explored in the next section.

\subsection{Five scenarios exploring the influence of perception on aggre- gation}

In the previous section it was demonstrated that aggregate cohesiveness depends not only on how much information agents have, but also on whether or not this sample of information provides a sufficiently accurate characterisation of relevant properties of the aggregate as a whole. Since the information received by animals depends on the functioning of their perceptions ( represented in the PCA model by perceptual fields) it is expected that the shape of the perceptual field in the models and, by extension, perceptual abilities in real aggregates will, in turn, mediate the information that is available to animals. This section will consider the ways in which the shapes of perceptual fields determine both the number and location of the perceptible neighbours for each agent in a group and how this, in turn, affects aggregate cohesiveness.

Prior to running the five scenarios, it was hypothesised that the shape of the perceptual fields of the agent would determine which and how many perceivable neighbours the agent would have at each time step. If a particular perceptual field shape reduces or increases the number of neighbours, or if that perception influences the type of neighbours (e.g., nearest neighbours, distant neighbours) that the agent tends to have at each time step, this will also affect the behaviour of the agent and, in turn, the behaviour of the aggregate. 


\subsubsection{Model setup and methodology}

Five different perceptual field scenarios were considered. Models in each scenario were the same as models used in Chapter 4 (see Algorithms 1 and 2), but with changes to the perceptual fields and attention algorithms, as described below.

Perceptual field scenarios For each of the five scenarios, all agents in the scenario were assigned perceptual fields of a specified shape, as follows (see also Figure 26):

- sphere scenario: a full sphere with a radius of 40 units

- angle scenario: two half spheres with radii of 40 units, angled 13 degrees off the normal, in front of the agent (the same as the $D$. rerio visual perceptual fields in Chapter 4)

- front scenario: a single half sphere with a radius of 40 units straight in front of agent

- wide scenario: two cones, each with a height of 40 units and an angle of 90 degrees extending out from the sides of the agent

- narrow scenario: two cones, each with a height of 80 units and an angle of 45 degrees extending out from the sides of the agent

The sphere and angle fields had the same volume, and the front, wide and narrow fields all had volumes half that of the sphere and angle fields. Note that these are the only perceptual fields of the agents- the lateral line fields of agents in Chapter 4 have been removed.

Attention algorithms For each perceptual field scenario, 3 attention algorithms (algorithms used to choose which agents would be processed by the cognitive processing algorithms) were considered: the all neighbours attention algorithm, which attended to all of the neighbours perceived by the agent, the 4 random neighbours attention algorithm, which picked four neighbours at random from all of the agents perceived by the agent and the 4 nearest neighbours attention algorithm, which picked the four nearest neighbours perceived by the agent (not necessarily the actual 4 nearest neighbours of the agent). Based on results from the experiments in Section 5.2, 4 was considered to be a intermediate value with respect to its influence on aggregate behaviour. 


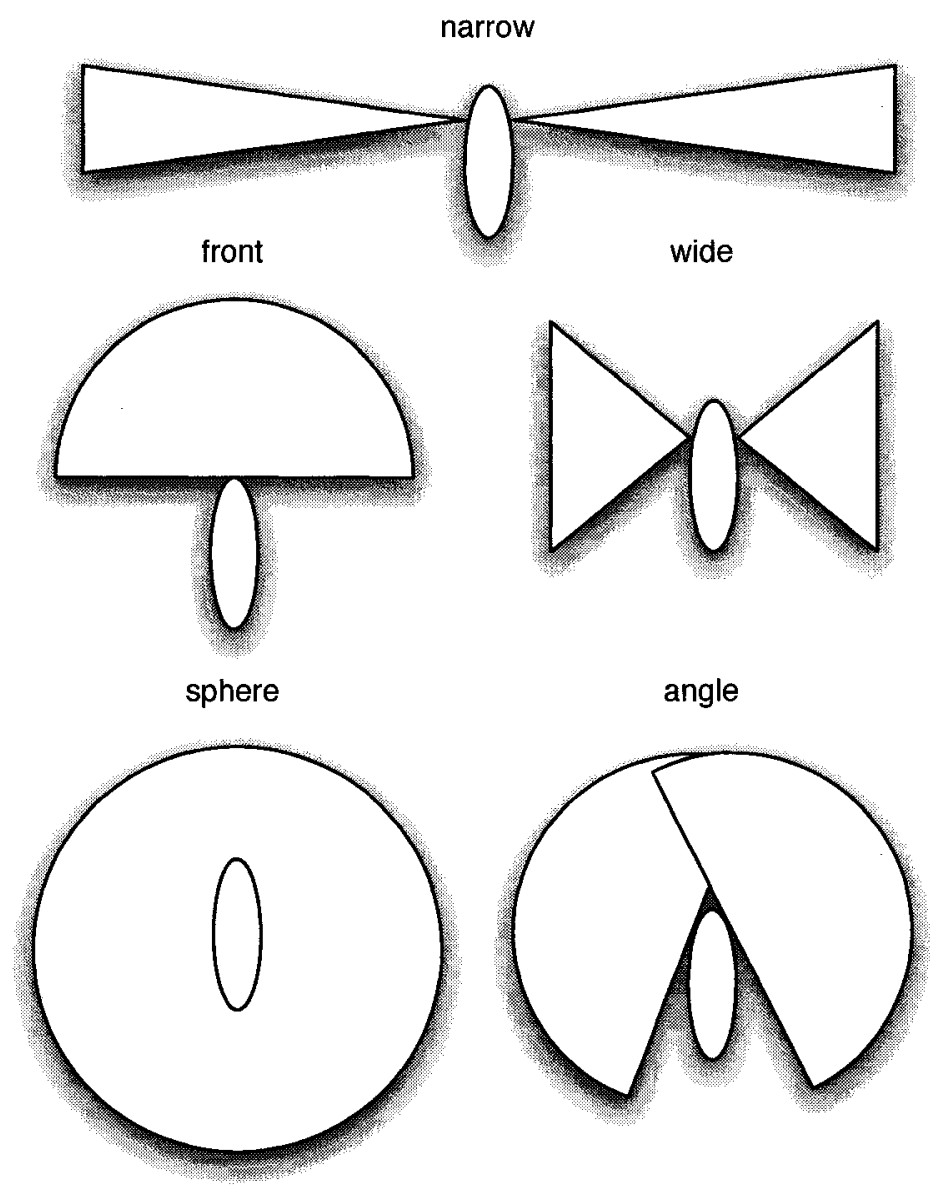

Figure 26: Shape of the 5 perceptual fields (not to scale). 
$\operatorname{Timestep}(O, \lambda)$

1 for each agent in $\lambda$

2 do $L \leftarrow$ PERCEPTION $(O)$

$3 \quad L^{\prime} \leftarrow \operatorname{ATTENTION}(L)$

$4 \quad I \leftarrow$ COGNITIVE-PROCESSING $\left(L^{\prime}\right)$

$5 \quad \operatorname{ACTION}(I)$

$\operatorname{ATTENTION}(L)$

$1 \quad L^{\prime} \leftarrow \operatorname{MERGE-LISTS}(L)$

$2 \triangleright$ the appropriate PICK-NEIGHBOURS procedure (below) is called for each scenario

$3 \quad L^{\prime} \leftarrow$ PICK-NEIGHBOURS $\left(4, L^{\prime}\right)$

4 return $L^{\prime}$

PICK-NEIGHBOURS-RANDOM $\left(N, L^{\prime}\right)$

1 return $\operatorname{RANDOM}\left(N, L^{\prime}\right)$

PICK-NEIGHBOURS-NEAREST $\left(N, L^{\prime}\right)$

1 return $\operatorname{NEAREST}\left(N, L^{\prime}\right)$

PICK-NEIGHBOURS-ALL $\left(N, L^{\prime}\right)$

1 return $L^{\prime}$

Algorithm 4: Agent behaviour during one timestep for the perceptual field models. Model algorithms are the same as those described in Chapter 4, Algorithms 1 and 2, with the exception of the attention algorithm, as described here.

Additional combinations of movement parameter settings (maximum speed and maximum turning angle) and one cognitive setting (preferred space) were also chosen, in order to more fully explore the parameter space of the scenario models (see Table 13 for more details on these additional parameter settings). Cognitive weights were fixed at $w_{c}=1, w_{v}=1$ and $w_{s}=3$, which, based on results from Chapter 4 , were considered to be values that would not overshadow the influence of other parameters (the influence of cognitive weights on aggregate behaviour will be investigated in Chapter 6). All possible combinations of the parameter values resulted in 240 parameter combinations.

Model runs As in Section 5.2, 10 model runs of 100 timesteps each were run for each parameter combination in order to determine if perceptual field shape affected the behaviour of the 


\begin{tabular}{ll} 
model parameter & values - \\
\hline perceptual field shape & (sphere, angle, front, wide, narrow) \\
attention algorithm & (nearest neighbours, all neighbours, random neighbours) \\
number of neighbours attended to & 4 \\
$s_{\max }$ & $0.5 \mathrm{u} / \mathrm{t}, 2.0 \mathrm{u} / \mathrm{t}$ \\
$t \theta_{\max }$ & $0.78 \mathrm{rads}, 3.10 \mathrm{rads}$ \\
$s p a c e_{\text {pref }}$ & $2 \mathrm{u}, 10 \mathrm{u}$ \\
$w_{c}$ & 1 \\
$w_{v}$ & 1 \\
$w_{s}$ & 3 \\
starting position radius $\left(r_{s p}\right)$ & $1 \mathrm{u}, 40 \mathrm{u}$
\end{tabular}

Table 13: Model parameters for the perceptual field scenarios and the values used for these parameters $(\mathrm{u}=$ units of distance, $\mathrm{t}=$ timestep).

aggregate in a consistent manner across parameter settings. As for the previous models, for each model run, at each time step, number of stragglers (individuals not within 20 units of another) number of groups (where a group is a set of agents that are within 20 units of another agent in the set) and number of clusters (number of groups and number of stragglers) were calculated.

Analysis The main aggregate measure considered when analysing the results of the model runs was number of clusters. As stated in Section 5.2, this aggregate measure provides an indication of overall aggregate cohesiveness (with low cluster numbers indicating high cohesiveness), and so was considered to be the most basic and essential aggregate measure. If the average maximum value for the number of clusters for a particular parameter setting was less than 2 , this indicated that in at least one of the 10 model runs for a particular setting, the aggregate was still a cohesive group after 100 time steps (with a value of 1 indicating that all 10 runs were cohesive). Values of 2 or greater indicated that for no model runs of that setting did the aggregate remain together as a cohesive single group during the 100 timesteps of the model run. As well, for each timestep and agent, the model recorded the number of other agents perceived and whether or not any of these were actual nearest neighbours.

Neighbour data was analysed for each scenario by first adding up all of the neighbours perceived in a given time step across agents, then adding up all of the neighbours perceived that 
were actually nearest neighbours, and finally counting how many agents perceived no neighbours in a given timestep. Then, maximum aggregate measure and neighbour data values for each run were calculated and averaged across runs and these collected results further analysed across parameter settings to determine how the number and type of neighbours influenced the aggregate behaviour of the model. Model results were analysed to determine if there were consistent patterns for each aggregate measure across parameter combinations, with particular emphasis on correlations between parameter values and aggregate measures. Correlation was calculated using Pearson's correlation coefficient, $r$. Differences between values were confirmed using a paired t-test (for comparisons of two values across parameter combinations).

\subsubsection{Model results}

Field volume and cohesion As noted in Section 5.3.1, sphere and angle fields have the same volume, and wide, side and angle fields all have volumes half this size. There was a strong but only weakly significant correlation $(n=5, r=0.86, t(3)=2.92,0.05<p<0.10)$ between volume of field and total number of parameter combinations with full cohesion across parameter settings with that perceptual field (cohesion ${ }_{\text {tot }}$ ), with full cohesion defined as the aggregate remaining cohesive for all 100 timesteps of all ten runs. Volume of perceptual field was also a poor predictor of aggregate cohesiveness in terms of total number of clusters across all parameter

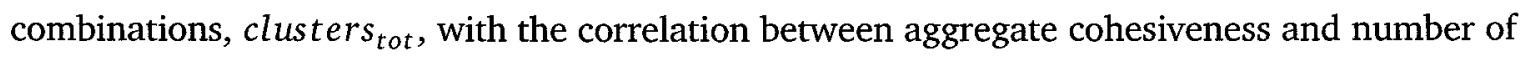
clusters weak and not significant $(n=5, r=-0.47 t(3)=-0.92 p>0.1)$. With respect to this measure, the wide field, despite being the same volume as the narrow and front fields, and half of the volume of the front and sphere fields, had the smallest number of total number of clusters across all parameter sets (see Table 14).

A one way ANOVA blocked across parameter combinations showed a significant difference across the five scenarios, with respect to number of clusters (5 blocks, 48 values per block, $F(4)=5.973, p<0.001$ ). A follow up Tukey test showed that the mean number of clusters for the wide, sphere and angle scenarios were not significantly different from each other, but that the mean number of clusters for the front scenario was significantly different from the 


\begin{tabular}{llllll} 
Perceptual field & clusters $_{\text {tot }}$ & cohesion $_{\text {tot }}$ & none $_{\text {perceived }}$ & tot $_{\text {perceived }}$ & $v_{\text {pf }}$ \\
\hline angle & 69.3 & 29 & 0 & 848.39 & 201061 \\
front & 107.4 & 13 & 172.4 & 590.30 & 100530 \\
narrow & 154 & 15 & 558.4 & 215.25 & 100530 \\
sphere & 72 & 29 & 0 & 867.28 & 201061 \\
wide & 54.1 & 23 & 62 & 479.25 & 100530
\end{tabular}

Table 14: Cohesion of aggregates with different perceptual field shapes: total of average maximum cluster values across all parameter combinations (clusters $s_{t o t}$ ), total number of parameter combinations with full cohesion across all 10 runs ( cohesion $_{\text {tot }}$ ), mean number of agents that perceived no neighbours (none $_{\text {perceived }}$, mean value of the total perceived neighbours for each parameter combination to $t_{\text {perceived }}$ and perceptual field volume in $\mathrm{cm}^{3}\left(v_{p f}\right)$.

four other scenarios, as was the mean number of clusters for the narrow scenario $(0.05$ level of significance).

Perceived agents and cohesion To gain a better understanding of why perceptual field volume alone does not predict cohesion, perceived agent data was also considered. The none $e_{\text {perceived }}$ data showed that, for the sphere and angle fields, all agents always perceived at least one other agent in the aggregate in every timestep, whereas for the narrow and front fields, there were no parameter settings where all agents perceived at least one other agent across all timesteps. For the wide perceptual field there were only 3 out of 48 parameter combinations where all agents always perceived at least one other agent across all timesteps. Agents in the narrow and front scenarios perceived relatively small numbers of agents when compared to agents in the other perceptual field shape scenarios and had more agents who perceived no other agent in the aggregate (see Table 14) .

Volume of perceptual field was strongly but not significantly correlated with the total number of agents perceived, tot $t_{\text {perceived }}(r=0.80 t(3)=2.31, p>0.1)$, and neither volume nor numbers of agents perceived were strongly correlated with number of clusters (across all parameter combinations, $n=240, r=-0.41, t(238)=-6.93, p<0.01$ for correlation between number of agents perceived and number of clusters and $r=-0.22 t(238)=-3.48, p<0.01$ for correlation between volume of perceptual field and number of clusters). This is because, as was stated above, in the sphere and angle cases there were never times when only a small number of agents 
were perceived - frequently all possible agents that could be perceived were being perceived. Nonetheless, aggregate cohesion still failed for some parameter combinations of the sphere and angle fields - most notably, in 12 (angle) and 11 (sphere) of the 16 parameter combinations where the nearest neighbour attention algorithm was being used.

\subsubsection{Discussion}

Importance of field shape Generally speaking, the behaviour of the model across parameter settings was predictable based not on the volume of the perceptual field but rather on the nature of information available to each agent, which in turn was predictable based on the shape of their perceptual fields. When agent fields were shaped such that they provided a sufficient approximation of a random sample of a 360-degree view of the entire aggregate (sphere, angle and wide field), agents could approximate the random sampling of all individuals in the aggregate and successfully aggregate, even with small amounts of information, just as agents in Section 5.2 could aggregate successfully with only one piece of information, randomly sampled from the aggregate. In contrast, when agent fields were poor approximations of a 360-degree, full aggregate field, agents were unable to approximate the random sampling of individuals in the aggregate and over time the aggregate did not remain cohesive, even with relatively large amounts of information. Aggregates also lost cohesion when using the nearest neighbour algorithm, again, even if large amounts of information were available to agents.

Narrow and front field cohesion failure With respect to why particular shapes of field failed to allow a sufficient random sample, agents in the narrow and front scenarios either perceived relatively small numbers of agents when compared to agents in the other perceptual field shape scenarios (in the case of the narrow side field), or had more agents who perceived no other agent in the aggregate (in the case of the front field). Both of these results can be related to edge effects.

In the case of the narrow side field, it can be speculated that large parts of the field pass entirely out of the aggregate, thus explaining the very low numbers of agents being perceived by the agents in models with this perceptual field (see Figure 27). The small amount of information 
available means that agents cannot attain cohesion using the nearest neighbour algorithm, which requires large amounts of information to generate aggregate cohesion. As well, it can be speculated that, again because of the field shape, the information obtained by individuals fails to approximate a random sample of the agents. This in turn means that agents are unable to attain cohesion using the random neighbour algorithm.

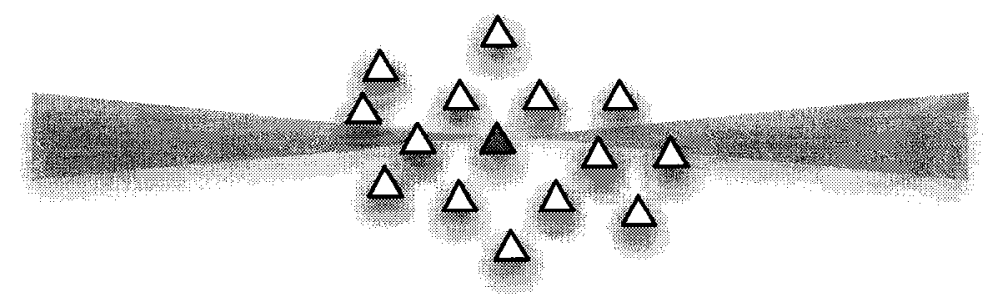

Figure 27: Narrow perceptual fields (shaded grey) can easily pass out of the densest part of the aggregate, even if they have a relatively large area or volume.

Unlike agents in the wide scenario, in the front field case, agents in the front of the aggregate cannot perceive those agents that are behind and to the left, and behind and to the right. These differences are reflected in the fact that, overall, more agents are perceived in the front field case, compared with the wide side case (see Figure 28), but there are also more agents that cannot perceive any other agent (see Figure 29). Again, although in theory one piece of information per agent can be sufficient for agents to aggregate, perceiving only a few agents at a time and also having relatively large numbers of agents not perceiving any other agents over an extended period of time will result in a loss of aggregate cohesion because agents cannot maintain a sufficiently accurate picture of the aggregate over time.

Sphere and angle field cohesion failure Since the sphere and angle perceptions also showed low cohesion in some cases (specifically when using the nearest neighbour attention algorithm) but also frequently had information about all of the agents in the aggregate, low information or poorly positioned perceptions alone cannot be used to predict cohesion. To understand what influences cohesion in these other cases, the attention algorithm must be considered. As was shown in Section 5.3.2, if the right agent is chosen from the group at each time step, an agent only needs information about a single other member of the aggregate in order for the aggregate 

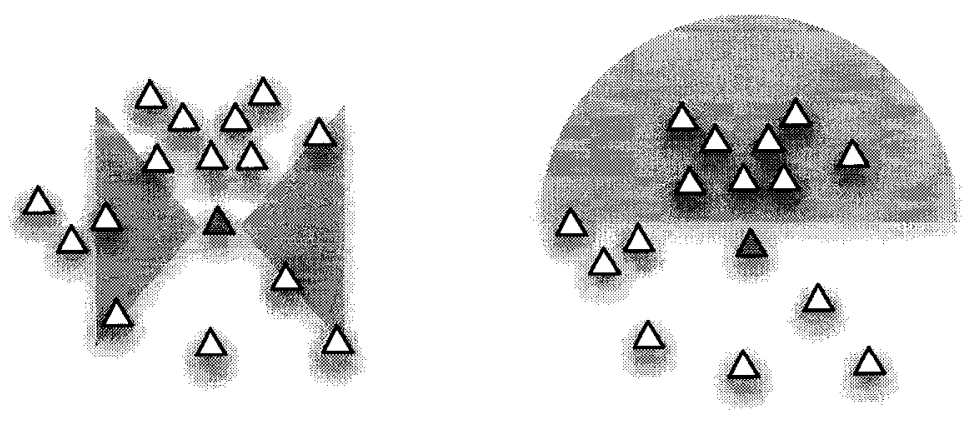

Figure 28: Two agents perceiving the same aggregate will receive different information, even if the volume or area covered by their perceptual fields (shaded grey) is the same. In this case, the agent on the left, with the wide fields, perceives fewer agents than the agent on the right, with the front field, but has a more accurate picture of the aggregate.
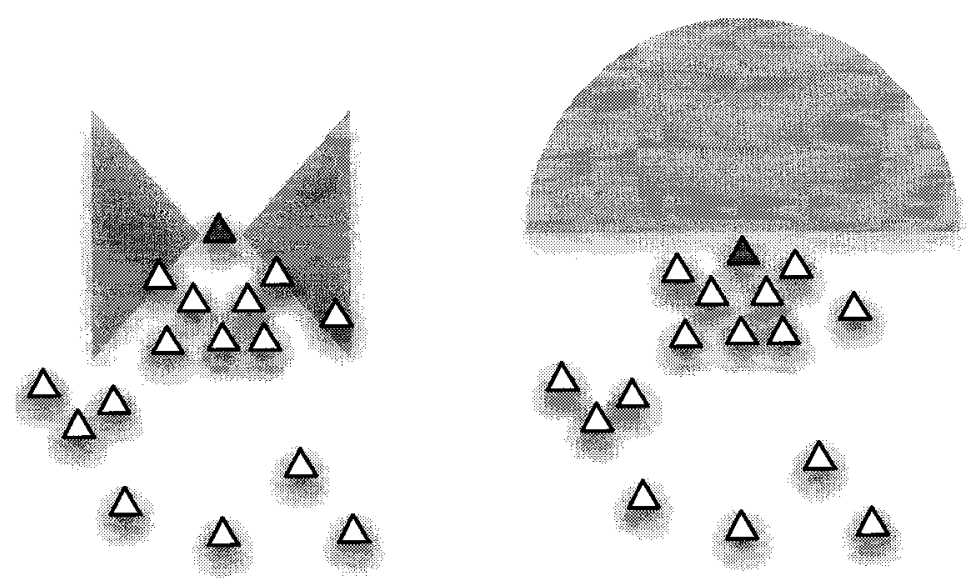

Figure 29: The agent on the left, with the wide fields (shaded grey) perceives some agents, while the agent on the right completely fails to perceive agents. 
to be successful in cohesion. Despite there being only one agent involved, the group does not disintegrate. In the case of the nearest neighbour attention algorithm, however, the group breaks up into smaller and smaller groups.

This disintegration into subgroups is understandable if the nearest neighbour algorithm is seen as functionally equivalent to limiting the perceptual field of the agent to a sphere with a radius of the farthest nearest neighbour, and then perceiving all agents within that perceptual field. In such a situation, the effective perceptual field is too small to obtain an accurate picture of overall group behaviour and the aggregate loses cohesion. In the case of the sphere and angle cases, all of the actual nearest neighbours of the agents were successfully perceived, which resulted in these aggregates falling into the nearest neighbour situation discussed in Section 5.3.2, and the aggregate losing cohesion. In the case of the wide field scenario, however, the perception of the agents was imperfect. As a result, agents frequently picked as 'nearest neighbours', agents that were not their actual nearest neighbours (see Figure 30). As a result, these cases had a resemblance to the random scenario discussed in Section 5.2, and consequently had better cohesion.

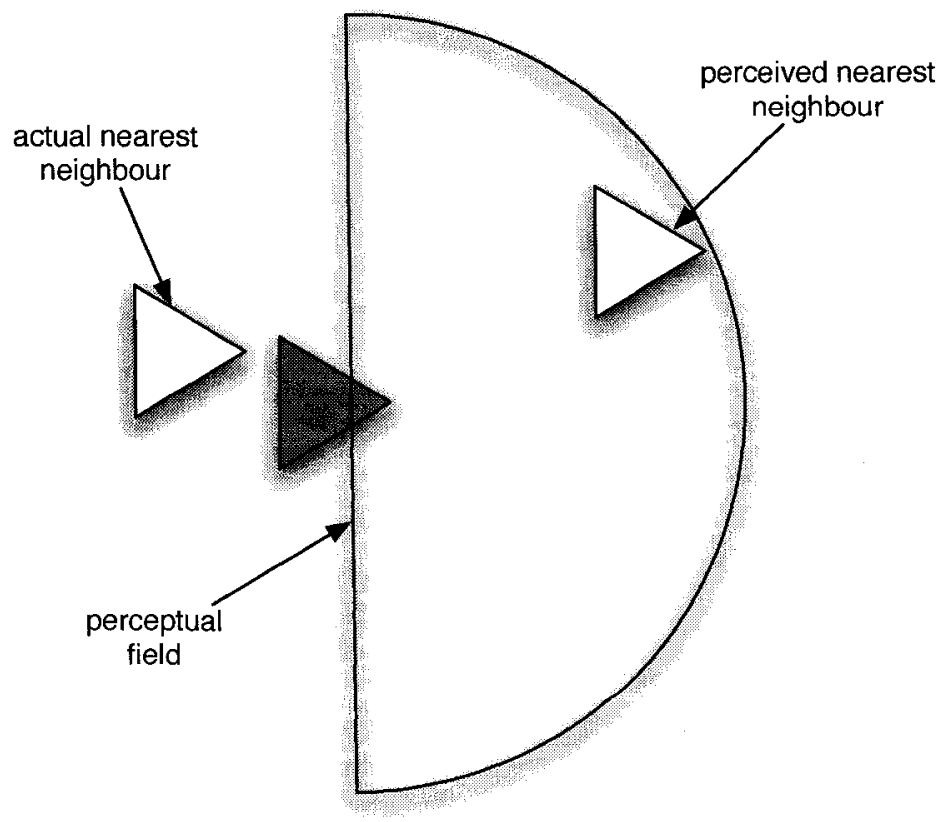

Figure 30: Perceived nearest neighbours and actual nearest neighbours. 
Existing models have typically used either a sphere field or variants on the angle field (Viscido et al., 2005, 2007; Hemelrijk and Hildenbrandt, 2008). These field shapes are frequently used in conjunction with attention algorithms that either use all agents perceived to determine movement (Couzin et al. 2002) or use some variant of the nearest neighbour algorithm (Huth and Wissel, 1992) to pick agents for movement determination. Based on the results in this section, variants on the angle field shape can be expect to contribute to high cohesion in the aggregate so long as the nearest neighbour algorithm is not also being used. However results in this section support the importance of basing the shape of the perceptual field on the actual perceptual abilities of the animal being considered, because, as was shown by the interaction of the wide field with the nearest neighbour algorithm, aggregate behaviour is influenced by both perceptual field shapes and the attentional algorithms animals use to process the information received by their perceptions.

\subsection{Chapter summary}

In Chapter 5 the role of perception in aggregation was examined by considering how the shape of the perceptual field influenced aggregate cohesiveness. Preliminary theoretical work was done in order to determine what amount of information was required in order for aggregation to occur, and how the amount of information available to individuals in an aggregate influenced the overall behaviour of the aggregate. The results of this investigation determined that there was an interaction with the attention algorithm and the amount of information required. For one of the attention algorithms considered, the random neighbour algorithm, individuals required a single piece of randomly collected information in order for the maintenance of aggregation. The other attention algorithm, the nearest neighbour algorithm, did not promote stable aggregation, even when up to half of the aggregate was detected by each individual.

In the second part of the chapter an investigation of the role of perceptual field shape in aggregate cohesion was carried out, with attention given to the results of the first investigation. It was determined that the perceptual field shape, more significantly than the volume, determined the type of information received by agents, which, in turn, determined aggregate cohesiveness. 
Thus, fields which provided information that reflected a more accurate approximation of the actual aggregate structure were more successful at maintaining cohesion. An interaction between the perceptual algorithm and the attention algorithm was also observed, with one particular shape, the wide field shape, operating successfully using both attention algorithms, while other field shapes, including ones with greater volume, were less successful using the nearest neighbour algorithm, relative to the random neighbour algorithm. In Chapter 6 , the role of cognition in aggregate behaviour will be explored further, with an emphasis on how cognitive parameters influence polarity and density of the aggregate. 


\section{Chapter 6}

\section{The influence of individual cognition and movement on aggregate movement, alignment and volume}

The PCA framework emphasises the importance of explicitly modelling each of the perception, cognition and action properties of aggregating animals, with a focus on incorporating experimental data into the model for each of these three components. Chapter 6 will underscore the importance of including and realistically modelling all three components by investigating how cognitive and movement parameters interact with perception to produce variability in aggregate behaviour. Specifically, after considering the question of cognitive requirements for aggregation, the chapter will compare two perceptual scenarios- one a prey analogue and one a predator analogue- with a focus on quantifying how cognitive parameters (space pref $_{\text {and }} w_{c}$, $w_{v}$ or $\left.w_{s}\right)$ and movement parameters $\left(s_{\max }\right.$ and $\left.t \theta_{\max }\right)$ influence particular aggregate properties like aggregate volume, aggregate speed, aggregate movement angle and aggregate alignment in each of the two scenarios. In the process it will be shown that an aggregate is a complex system, in that individual behaviours, represented by model parameters, have non-additive effects on aggregate behaviour.

\subsection{Types of cognition required for aggregation}

The PCA framework takes the abstract concepts of attraction and repulsion and converts them from external forces (back) into cognitive algorithms. Although, as noted in Chapter 4, it is 
currently difficult to determine what cognition is taking place in aggregating animals, previous aggregate models have made some contributions to understanding the necessary and sufficient cognitive (and movement) abilities for aggregation. Vicsek et al.'s simple particle model (1995) demonstrated that in a closed space, with individuals moving at a fixed speed, the ability to observe the alignment of conspecifics and align one's own body with these conspecifics could be sufficient to generate coordinated movement behaviour. Parr's (1927) argument that attraction and repulsion responses are also necessary for aggregate behaviour was supported by Grégoire et al. (2003), who argued that in an unenclosed space, simple alignment will not suffice for maintenance of the aggregate, and that abilities to respond to stimuli by repositioning (such that surrounding animals are neither too close nor too far away) are also necessary. Grégoire et al. (2003) also suggest that the ability of individuals to match velocity (speed and direction of conspecifics) is necessary if the aggregate as a whole is to move through space, rather than remaining in place.

These results support the idea that aggregating species must possess perceptual abilities that allow them to detect the spatial positions and alignments of conspecifics, and cognitive and movement abilities that allow them to react to this information by adjusting their movements in such a way that they position themselves in specific ways relative to these conspecifics. In order for the aggregate to move through space the results discussed above also suggest that agents must be able to detect in some way the velocities of conspecifics and then match their velocities with those of conspecifics. Taken together, these results suggest that some form of all of these cognitive abilities - attraction, repulsion and velocity matching (speed and alignment) - need to be included in species-specific models.

The relative strength of each of these cognitive factors is less clear, however, as is the way in which these factors interact. Consequently, within the context of basic perceptual and cognitive requirements, there remains the question of how an animal combines attraction, alignment and repulsion responses when determining movement, how these responses interact with movement abilities and how this, in turn, influences aggregate behaviour. A more detailed understanding of these influences and interactions will allow for more specific predictions about aggregate behaviour for particular aggregates in specific circumstances. 
Following from this, there are a number of hypotheses about cognitive and movement parameters that can be tested by an aggregate model. The first of these hypotheses is concerned with whether or not any of the aggregating behaviours- attraction, repulsion and alignmentare necessary or sufficient for generating aggregate behaviour and under what circumstances they do generate aggregate behaviour. Other hypotheses concern the ways in which these three behaviours influence aggregate properties other than cohesion, and also whether or not movement abilities interact with these cognitive factors to influence aggregate properties.

\subsection{The aggregate as a complex system: Predicting interactions be- tween cognition, movement and perception}

It is understood that many biological systems are complex systems, although what precisely constitutes a complex system can be difficult to define. Bossomaier and Green (2000, p. 2) note that, "[a]lthough it might be intuitively apparent that a system is complex, defining complexity has proved difficult to pin down with numerous definitions on record." However, they go on to state that " $[\mathrm{A}]$ major source of complexity is the interaction of many autonomous, adaptive agents", something which is frequently the case in natural systems and IBSEM models of these systems. Generally speaking, in a complex system the emergent properties of the system as a whole are not predictable in a straightforward manner from the values of individual or isolated parameters of the system. This is because the parameters in a complex system interact, and the behaviour of the system as a whole is a result of these interactions, rather than being the result of an additive effect of each of the parameters by themselves. For the purpose of this thesis, it will be assumed that the presence and number of non-additive effects and high-level interactions among parameters are indicative of the level of complexity of a system and if observed in a system provide evidence that that system is a complex system.

With respect to the behaviour of such a system, an extreme prediction would be that every individual (or component-level) property, represented in the model by parameters, would influence every system-level measure, and also that these properties would fully interact in their influence of each system-level measure, such that knowledge of the specific value of all 
individual parameters would be required to predict any aspect of the behaviour of the system. If an aggregate (and by extension, aggregate model) was found to be this complex, predicting the behaviour of the aggregate based on values of model parameters would be difficult in advance of running the model, and even then, once analysing the model, only predictable if all values of parameters were known for a particular case. Viscido et al. (2007) found interactions between all social forces in their fish aggregate model, supporting this possibility.

In contrast to this extreme prediction, however, what might be referred to as naïve predictions - simple predictions that hypothesise straightforward connections between a property at the individual-level and similar properties at the aggregate-level — still have value. This is because proving or disproving them provides information about the behaviour of the system. They also have value because they provide an opportunity to demonstrate that some interactions may, in fact, be easily predictable, and mainly dependent on one model parameter, even if others are more complex. One useful task, from a modelling perspective, is to investigate these naive predictions and determine which properties interact and which do not. This will be considered with respect to aggregate behaviour in the remainder of the chapter.

Taking a naïve point of view, it might be predicted that in an aggregate there will be a straightforward match between individual and group properties, so that, for example, individual properties that affect speed will affect group speed measures (i.e., as individuals change their speed, the speed of the aggregate also changes), individual properties involving spacing will affect group spacing and volume measures and individual properties that involve angles will affect group angle measures. It might also be naïvely predicted that there will be a simple relationship between the value of parameters and the value of those group measures that they are dependent on, such that the extreme values of the group measures (either low or high) will be matched with the extreme values (either low or high) of the parameter (i.e., as the speed of individuals within the aggregate increases, the speed of the aggregate as a whole will increase). These naïve predictions are presented in Table 15, which shows, for each aggregatelevel measure (dependent variable, along the top of the table), the predicted influence from each of the 6 individual-level measures (independent variables, down the side of the table). 


\begin{tabular}{lllllllll} 
individual & group & \multicolumn{10}{l}{} \\
\hline & $v_{\text {simp }}$ & $\exp$ & $p$ & $n_{\text {groups }}$ & $n_{\text {strag }}$ & $n_{\text {clust }}$ & $s_{\text {agg }}$ & $t \theta_{\text {agg }}$ \\
space $_{\text {pref }}$ & + & + & 0 & + & + & + & 0 & 0 \\
$w_{c}$ & - & - & 0 & - & - & - & 0 & 0 \\
$w_{v}$ & 0 & 0 & + & 0 & 0 & 0 & 0 & + \\
$w_{s}$ & + & + & 0 & + & + & + & 0 & 0 \\
$s_{\max }$ & 0 & 0 & 0 & 0 & 0 & 0 & + & 0 \\
$t \theta_{\max }$ & 0 & 0 & 0 & 0 & 0 & 0 & 0 & + \\
\hline
\end{tabular}

Table 15: Predicted relationship between individual properties (independent variables) and aggregate properties (dependent variables). See Table 5 and Table 7 for a description of parameters and group measures).

The remainder of this chapter will test these naïve predictions by exploring how varying cognitive and movement parameters (corresponding, by analogy with actual aggregating animals, to possible biological differences) influences the behaviour of the aggregate model and, by extension, the behaviour of different animal aggregates. Multivariate analysis will be used to gain insight into the more complex interactions of these model parameters and determine which parameters do and do not interact to produce aggregate behaviours commonly observed in actual aggregates.

\subsection{Modelling the interaction between cognition, movement and perception}

This section will consider, for two separate scenarios- the prey perceptual scenario and the predator perceptual scenario- how cognitive behaviours, represented by the attraction, repulsion and alignment responses of agents and the preferred spacing of agents, interact with agent movement abilities, represented by $s_{\max }$ and $t \theta_{\max }$, to produce group aggregate properties. The prey scenario is presented as a theoretical analogy to vision in prey animals like schooling fish, flocking birds and herding ungulates. The predator scenario is presented as a theoretical analogy to bifocal vision in predator animals like birds of prey, canines and dolphins. It is expected that 
the prey perceptual scenario will result in more reliable and predictable aggregate behaviour, because this scenario is more representative of the typical prey aggregates found in nature.

\subsubsection{Setup and methodology}

Model structure The architecture of the model used as the basis for these two scenarios has been discussed in Chapter 4 (Algorithms 1 and 2 and Chapter 5 (Algorithm 4. However, like the scenarios in Chapter 5 , agents in the Chapter 6 scenarios do not have side (lateral line) fields. Two of the perceptual field scenarios from Chapter 5 were chosen for the perceptual scenarios in Chapter 6: the side angle field, where agents have almost 360 degree vision (with a blind spot in the back and a small blind spot at the nose) and the front field, where agents have only front vision (see Figure 26). The angle field scenario is presented as the theoretical analogy to prey vision and the front field scenario is presented as a theoretical analogy to predator vision.

To allow for a direct comparison of the influence of the perceptual field shape on aggregate behaviour, attentional, cognitive processing and movement algorithms were the same for the two scenarios. In both scenarios, agents use the random neighbour attention algorithm from Chapter 5 , with up to 4 random neighbours chosen from the agents detected by the perceptual fields ( $N=4$ in Algorithm 4).

Model parameters Unlike in previous models from Chapters 4 and 5, multiple values of both cognitive and movement parameters, along with the two perceptual field shapes, were used in order to investigate the interaction between perception, cognition and movement parameters. The values used for each parameter are listed in Table 16. All combinations were used, with the exception of combinations where $w_{c}, w_{v}$ and $w_{s}$ all equalled 0 , which would have resulted in no external influence on agent behaviour. As in Chapter 5, each of the resulting 208 parameter combinations were run 10 times, with 30 agents, for 100 timesteps.

Analysis Results were analysed by considering 8 aggregate-level measures: 2 aggregate volume measures (simple volume and expanse), an aggregate alignment measurement (polarisation), 3 aggregate cohesiveness measures ( $n_{\text {groups }}, n_{\text {strag }}$ and $n_{\text {clust }}$ ), a measure of the distance the 


\begin{tabular}{ll} 
parameter & values \\
\hline$F$ & (prey, predator) \\
space $_{\text {pref }}$ & $(2,10)$ units \\
$w_{c}$ & $(0,1,3)$ \\
$w_{v}$ & $(0,1,3)$ \\
$w_{s}$ & $(0,1,3)$ \\
$s_{\max }$ & $(2,10)$ units/timestep \\
$t \theta_{\max }$ & $(0.78,3.00)$ radians
\end{tabular}

Table 16: List of parameter sets used: For each perceptual field $(F)$, different values for each parameter were used to explore the parameter space of the model, resulting in 208 parameter combinations.

aggregate moved in a timestep (aggregate speed) and a measure of the movement angle of the aggregate (aggregate turning angle). These aggregate measures are described in Chapter 4, Section 4.4.1.

These measures were calculated for each time step of each run, and then the maximum value of each of the aggregate measures for the run was used as a representative measure for the entire run. To obtain an overview of the model behaviour, the maximum values for all runs with a particular parameter combination were then averaged to provide a single value summarizing all runs with a particular parameter combination (see Figure 21 in Chapter 4, for an explanation of this process). This resulted in 208 values for each measure, one for each parameter combination.

A comparison of aggregate behaviour between the two scenarios was carried out by calculating blocked ANOVAs with the maximum averaged parameter combination values. Then, for each scenario, a multivariate analysis of the six model parameters (referred to as factors in the context of the multi-way ANOVA and linear regression analysis) was done for each aggregate measure to determine which, if any, factors were not relevant for each aggregate measure and which, if any, factors did not interact with other factors. To further determine the contribution of each factor to each aggregate measure, a linear regression model was also calculated for each aggregate measure. For multi-way ANOVAs and linear regression, maximum values for all runs of all parameter combinations were used, resulting in 2080 values for each measure for these calculations. 
In order to determine which aggregate measures were correlated with each other, correlation coefficients were also calculated for the aggregate measures, using the maximum averaged values. This correlation was carried out because cognitive underpinnings of aggregating individuals, as represented by cognitive weights in this chapter, are not directly observable, but aggregate measures are. Furthermore, if aggregate measures are correlated with each other, the type of aggregates that are actually seen in nature can expect to be constrained based on this correlation. As well, since some explanation of the connection between aggregate behaviours and underlying cognitive measures will have been provided by the regression analysis, the reason behind a particular grouping of aggregate behaviours can be more fully explained by reference to the underlying cognitive and movement factors.

Lastly, to gain a better understanding of the necessary and sufficient cognitive behaviours for aggregation, results were considered where each of $w_{c}, w_{v}$ and $w_{s}$ were equal to 0 (with the exception of the case where all were equal to 0 ), effectively eliminating the influence of this behaviour from the resulting movement of the agent. For this analysis, number of runs where clusters were 1 or greater than 1 were counted to determine if there were any runs, in the absence of a particular behaviour, that remained fully cohesive at timestep $t=100$.

\subsubsection{Model results}

Overall comparison of prey and predator behaviour Blocked ANOVA calculations showed that the predator and prey scenarios differed on all aggregate measures except mean speed of aggregate. Averaged maximums and minimums for each of the eight aggregate measures are reported in Table 17 . This table shows that all aggregate measures had relatively large ranges across runs, with minimums and maximums of polarisation, mean speed, groups, stragglers and clusters corresponding closely with lowest possible minimum values and largest possible maximum values for these aggregate measures. Thus, although, as stated above, predator and prey models were significantly different when parameter combinations were blocked, the highest and lowest values of the aggregate measures across all parameter combinations were comparable, as shown in Table 17. 


\begin{tabular}{llll} 
measure & Prey (range) & Predator (range) & dif $f_{\text {apc }}$ \\
\hline$v_{\text {simp }}$ & $1.13 * 10^{3}--1.92 * 10^{7}$ & $1.26 * 10^{3}--6.17 * 10^{7}$ & yes, $\mathrm{F}=67.97, \mathrm{p}<0.001$ \\
$\exp$ & $3.02-58.78$ & $3.13-163.54$ & yes, $\mathrm{F}=107.10, \mathrm{p} 0.001$ \\
$p$ & $0.65-1.60$ & $0.619-1.59$ & yes, $\mathrm{F}=28.88, \mathrm{p}<0.001$ \\
$n_{\text {groups }}$ & $1-8.1$ & $1-7.8$ & yes, $\mathrm{F}=42.29, \mathrm{p}<.001$ \\
$n_{\text {strag }}$ & $0-30$ & $0-30$ & yes, $\mathrm{F}=10.981, \mathrm{p}=0.001$ \\
$n_{\text {clust }}$ & $1-30$ & $1-30$ & yes, $\mathrm{F}=19.89, \mathrm{p}<0.001$ \\
$s_{\text {agg }}$ & $0.20-9.99$ & $0.19-9.99$ & no, $\mathrm{F}=3.24, \mathrm{p}=0.073$ \\
$t \theta_{\text {agg }}$ & $0.21-2.95$ & $0.11-2.81$ & yes, $\mathrm{F}=10.20, \mathrm{p}=0.002$
\end{tabular}

Table 17: An overview of prey and predator behaviour: Ranges (min-max) across parameter combinations and whether or not the behaviour of the models differ when compared across all parameter combinations using a blocked ANOVA ( i $i f f_{a p c}$ ), $d f=1$ in all cases. Note that although the ranges may be similar, differences between scenarios are still significant because of the distribution of parameter combination values within these two extremes. See Table 7 for a definition of each aggregate measure.

Necessary and sufficient cognitive weights Six special cases (each with 3 subcases) where one or more of $w_{c}, w_{v}$ or $w_{s}$ equalled 0 were considered to determine if centring behaviours (strength determined by $w_{c}$ ), vector alignment behaviours (strength determined by $w_{v}$ ) and spacing behaviours (strength determined by $w_{s}$ ) were either necessary or sufficient for group cohesion (as measured by $n_{\text {clust }}$ ). For both the predator and prey scenario, when any one of $w_{c}, w_{v}$ or $w_{s}$ was 0 there was still at least one run where $n_{\text {clust }}$ remained 1 at $t=100$. (see Table 18). When both $w_{v}$ and $w_{s}$ or both $w_{c}$ and $w_{s}$ were 0 (disabling the behaviours associated with those weights), $n_{\text {clust }}$ was also still 1 in some runs. When both $w_{c}$ and $w_{y}$ were 0 , no runs maintained cohesion ( $n_{\text {clust }}>1$, regardless of the values of other model parameters). For the predator scenario, the number of times that $n_{\text {clust }}$ equalled one was smaller, reflecting the reduced cohesiveness of the predator aggregate, as discussed in Chapter 5 (see Table 18).

Multi-way (factorial) ANOVAs A multi-way ANOVA of the six factors for each of the 8 aggregate measures was carried out for the prey and predator scenarios (a $2 \times 3 \times 3 \times 3 \times 2 \times 2$ way ANOVA, total sample size $N=2160$ ). Analysis for the predator scenario (see Table 19, column 2) showed that, for every aggregate measure considered, each of the six factors (space $_{\text {pref }}, w_{c}$, $\left.w_{v}, w_{s}, s_{\max }, t \theta_{\max }\right)$ interacted significantly with at least two other of the six factors to influence 


\begin{tabular}{lll} 
weights & \multicolumn{2}{l}{ number of cohesive runs } \\
(out of a total of 80 possible runs) \\
$\left(w_{c} w_{v} w_{s}\right)$ & prey & predator \\
\hline 001 & 0 & 0 \\
003 & 0 & 0 \\
\hline 100 & 37 & 6 \\
300 & 45 & 31 \\
\hline 010 & 62 & 27 \\
030 & 8 & 0 \\
\hline 011 & 58 & 27 \\
013 & 47 & 24 \\
033 & 10 & 0 \\
031 & 7 & 0 \\
\hline 101 & 36 & 2 \\
103 & 39 & 1 \\
301 & 43 & 17 \\
303 & 39 & 3 \\
\hline 110 & 73 & 77 \\
130 & 67 & 32 \\
310 & 75 & 35 \\
330 & 78 & 62 \\
& &
\end{tabular}

Table 18: Aggregate Cohesion in the absence of centring, vector alignment and/or spacing. The number of cohesive runs is the number of runs where there was still a single cluster at $t=100$. 
behaviour. Except in the case of $v_{\text {simp }}$ and $t \theta_{\text {agg }}$, there were 5-way interactions between factors for all aggregate measures.

Results for the prey scenario were similar, except that $t \theta_{\max }$ did not interact with any other factor in $v_{\text {simp }}, s_{\text {agg }}$ and $\theta_{\text {agg }}$. There was also no main effect of $t \theta_{\max }$ in these cases. In other words, $t \theta_{\text {max }}$ did not influence $v_{\text {simp }}, \theta_{a g g}$ and $s_{a g g}$ and $w_{s}$ did not influence $n_{\text {strag }}$. For the prey scenario there were 5-way interactions for every measure except $\theta_{\text {agg }}$.

Linear regression For both the prey and predator scenario, a linear regression analysis of all six factors for each of the eight aggregate measures $(n=2080)$ resulted in relatively high error levels $\left(R^{2}<0.5\right)$, with two exceptions - group speed and group angle. However, all linear models were significant $(p<0.001)$, indicating that they provided a better than chance estimate of the aggregate measure in question, despite these error levels.

Standardised coefficients for these models (see Appendix B for coefficients) provided information on the direction and importance of the relationship for each factor and aggregate measure. For the prey scenario, $w_{c}, w_{v}, w_{s}$ and $s_{\max }$ were consistently large contributors for all of the aggregate measures, as indicated by the lack of O's in these rows in Table 20. The factor space $_{\text {pref }}$ was a significant contributor for all aggregate measures but $n_{g r o u p s}$ and group angle. The factor $t \theta_{\max }$ was only a significant predictor of $n_{\text {groups }}$, as indicated by 0 's across almost the entire row corresponding to $t \theta_{\max }$ in Table 20. See also Appendix B for the relative size of contribution by each of the individual measures.

For the predator scenario, as with the prey scenario (see Table 21), $w_{c}, w_{v}$ and $s_{\max }$ were consistently large contributors for all of the aggregate measures, although, in contrast to the prey model, an increase in $w_{c}$ led to an increase in aggregate volume. $w_{s}$ was a significant contributor for all but the aggregate volume measures, simple volume, and expanse. space pref was a significant but relatively small (see Appendix B) contributor for polarisation, $n_{\text {strag }}$ and $n_{\text {clust }}$. $t \theta_{\max }$ was a significant (but relatively small) component of the model for all but the $n_{\text {strag }}$. Again, see Appendix B for the relative size of contribution by each of the individual measures. 


\begin{tabular}{|c|c|c|}
\hline measure & prey & predator \\
\hline$v_{\operatorname{sim} p}$ & space $_{\text {pref }} \times w_{c} \times w_{v} \times w_{s} \times s_{\max }$ & $\begin{array}{l}w_{c} \times w_{v} \times w_{s} \times s_{\max } \\
w_{c} \times w_{v} \times s_{\max } \times t \theta_{\max } \\
\text { space }_{\text {pref }} \times w_{v} \times w_{s} \times s_{\max }\end{array}$ \\
\hline $\exp$ & $\begin{array}{l}\text { space }_{\text {pref }} \times w_{c} \times w_{v} \times w_{s} \times t \theta_{\max } \\
\text { space }_{\text {pref }} \times w_{c} \times w_{v} \times w_{s} \times s_{\max } \\
\text { space }_{\text {pref }} \times w_{c} \times w_{v} \times s_{\max } \times t \theta_{\max }\end{array}$ & $\begin{array}{l}w_{c} \times w_{v} \times w_{s} \times s_{\max } \times t \theta_{\max } \\
\text { space }_{\text {pref }} \times w_{c} \times w_{v} \times w_{s} \times s_{\max }\end{array}$ \\
\hline$p$ & $\begin{array}{l}w_{c} \times w_{v} \times w_{s} \times s_{\max } \times t \theta_{\max } \\
\text { space }_{\text {pref }} \times w_{c} \times w_{v} \times w_{s}\end{array}$ & $\begin{array}{l}w_{c} \times w_{v} \times s_{\max } \\
\text { space }_{\text {pref }} \times w_{c} \times w_{v} \times w_{s} \times t \theta_{\max } \\
\text { space }_{\text {pref }} \times w_{c} \times w_{s} \times s_{\max } \times t \theta_{\max }\end{array}$ \\
\hline$n_{\text {groups }}$ & $\begin{array}{l}\text { space }_{\text {pref }} \times w_{v} \times t \theta_{\max } \\
\text { space }_{\text {pref }} \times w_{c} \times w_{v} \times w_{s} \times s_{\max } \\
s_{\max } \times t \theta_{\max }\end{array}$ & $\begin{array}{l}w_{c} \times w_{v} \times s_{\max } \times t \theta_{\max } \\
\text { space }_{\text {pref }} \times w_{c} \times w_{v} \times w_{s} \times s_{\max }\end{array}$ \\
\hline$n_{\text {strag }}$ & $\begin{array}{l}w_{c} \times w_{v} \times s_{\max } \times t \theta_{\max } \\
\text { space }_{\text {pref }} \times w_{v} \times s_{\max } \times t \theta_{\max } \\
\text { space }_{\text {pref }} \times w_{c} \times w_{v} \times t \theta_{\max } \\
\text { space }_{\text {pref }} \times w_{c} \times w_{v} \times w_{s} \times s_{\max }\end{array}$ & $\begin{array}{l}w_{v} \times s_{\max } \times t \theta_{\max } \\
w_{c} \times w_{v} \times t \theta_{\max } \\
\text { space }_{\text {pref }} \times w_{c} \times w_{v} \times w_{s} \times s_{\max } \\
\text { space }_{\text {pref }} \times w_{s} \times t \theta_{\max }\end{array}$ \\
\hline$n_{\text {clust }}$ & $\begin{array}{l}w_{c} \times w_{v} \times s_{\max } \times t \theta_{\max } \\
\text { space }_{\text {pref }} \times w_{v} \times s_{\max } \times t \theta_{\max } \\
\text { space }_{\text {pref }} \times w_{c} \times w_{v} \times t \theta_{\max } \\
\text { space }_{\text {pref }} \times w_{c} \times w_{v} \times w_{s} \times s_{\max }\end{array}$ & $\begin{array}{l}w_{c} \times w_{v} \times s_{\max } \times t \theta_{\max } \\
\text { space }_{\text {pref }} \times w_{c} \times w_{v} \times w_{s} \times s_{\max } \\
\text { space }_{\text {pref }} \times w_{s} \times s_{\max } \times t \theta_{\max }\end{array}$ \\
\hline$s_{\max }$ & space $_{\text {pref }} \times w_{c} \times w_{v} \times w_{s} \times s_{\max }$ & $\begin{array}{l}w_{v} \times w_{s} \times s_{\max } \times t \theta_{\max } \\
w_{c} \times w_{s} \times s_{\max } \times t \theta_{\max } \\
\text { space }_{\text {pref }} \times w_{c} \times w_{v} \times s_{\max } \times t \theta_{\max } \\
\text { space }_{\text {pref }} \times w_{c} \times w_{s} \times t \theta_{\max }\end{array}$ \\
\hline$t \theta_{a g g}$ & $\begin{array}{l}w_{c} \times w_{v} \times w_{s} \times s_{\max } \\
\text { space }_{\text {pref }} \times w_{v} \times w_{s} \times s_{\max } \\
\text { space }_{\text {pref }} \times w_{c} \times w_{v} \times w_{s} \\
\text { space }_{\text {pref }} \times w_{c} \times w_{s} \times s_{\max }\end{array}$ & $\begin{array}{l}w_{v} \times s_{\max } \times t \theta_{\max } \\
w_{c} \times w_{v} \times t \theta_{\max } \\
\text { space }_{\text {pref }} \times w_{c} \times w_{v} \times w_{s} \\
\text { space }_{\text {pref }} \times w_{c} \times w_{s} \times s_{\max }\end{array}$ \\
\hline
\end{tabular}

Table 19: Factors that interact for each measure. This table reports significant interactions $(p<=0.05)$ that involve the highest number of unique interacting factors. Significant interactions that are subsets of these interactions are not shown. Interacting model parameters are joined by the $\times$ symbol. 


\begin{tabular}{lllllllll} 
& $v_{\text {simp }}$ & $\exp$ & $p$ & $n_{\text {groups }}$ & $n_{\text {strag }}$ & $n_{\text {clust }}$ & $s_{\text {agg }}$ & $t \theta_{\text {agg }}$ \\
\hline space $_{\text {pref }}$ & + & + & + & 0 & + & + & + & 0 \\
$w_{c}$ & - & - & + & - & - & - & + & + \\
$w_{y}$ & - & - & - & - & - & - & + & + \\
$w_{s}$ & + & + & + & + & + & + & - & + \\
$s_{\max }$ & + & + & - & + & + & + & + & - \\
$t \theta_{\max }$ & 0 & 0 & 0 & + & 0 & 0 & 0 & 0
\end{tabular}

Table 20: Prey: Relationship between individual properties and aggregate properties. A ' 0 ' represents no relationship, a '+' represents directly related and a '-' represents inversely related. See also Appendix B for the linear model coefficients.

\begin{tabular}{lllllllll} 
& $v_{\text {simp }}$ & $\exp$ & $p$ & $n_{\text {groups }}$ & $n_{\text {strag }}$ & $n_{\text {clust }}$ & $s_{\text {agg }}$ & $t \theta_{\text {agg }}$ \\
\hline space $_{\text {pref }}$ & 0 & 0 & + & 0 & + & + & 0 & 0 \\
$w_{c}$ & + & + & + & - & - & - & + & + \\
$w_{v}$ & - & - & - & - & - & - & + & + \\
$w_{s}$ & 0 & 0 & + & + & + & + & - & + \\
$s_{\max }$ & + & + & - & + & + & + & + & - \\
$t \theta_{\max }$ & + & - & + & + & + & + & 0 & +
\end{tabular}

Table 21: Predator: Relationship between individual properties and aggregate properties. A ' 0 ' represents no relationship, a ' + ' represents directly related and a ' - ' represents inversely related. See also Appendix B for the linear model coefficients. 
A comparison of the predictions in Table 15 with the results in Table 20 (see Table 22) shows that, for the prey scenario, 23 of the naive predictions were correct, out of a possible 48 . Although the naïve predictions frequently failed to predict the influence of particular parameters that did, in fact, influence the behaviour of a particular aggregate measure (the naive predictions failed to predict important factors 22 times), the naïve model was more successful in correctly predicting that a factor would influence a measure, incorrectly predicting influence only 2 times, and only once incorrectly predicting the direction of the influence. For the predator scenario, 14 of the naïve predictions were correct. Naïve predictions failed to predict the influence of particular parameters 26 times, and predicted the influence of a factor that had no influence 5 times, with an incorrect direction predicted 3 times.

\begin{tabular}{llllllll} 
& correct & \multicolumn{7}{l}{ incorrect (actual/predicted) } \\
& & $+/ 0$ & $0 /+$ & $+/-$ & $-/+$ & $-/ 0$ & $0 /-$ \\
\hline prey & $23 / 48$ & 14 & 2 & 0 & 1 & 8 & 0 \\
predator & $14 / 48$ & 17 & 5 & 2 & 1 & 9 & 0
\end{tabular}

Table 22: Actual and predicted relationship between individual properties and aggregate properties, for the prey and predator scenarios. Numbers in the first column (correct) indicate number of times the actual relationship matched the predicted relationship. Subsequent problems indicate the number of times a relationship was actually seen when a different relationship was predicted. The table header indicates which was the actual relationship and which was the predicted relationship (actual/predicted). A ' 0 ' represents no relationship, a ' + ' represents directly related and a '-' represents inversely related.

A comparison of Tables 20 and 21 (see Table 23) shows that there are some differences in linear models for these two scenarios. In 11 out of 48 cases, an individual property had an influence on an aggregate property in one scenario and not the other. However, there were only two cases where the influence of an individual property was reversed between the two scenariosthe influence of $w_{c}$ on simple volume and on expanse (the aggregate size measurements).

Correlation of aggregate measures With respect to correlation, for the prey scenario several aggregate measures were correlated with each other (correlation coefficient $r>0.5$, see Table 24). As expected, related measures (simple volume and expanse, $n_{\text {strag }}$, numbers of groups and $n_{\text {clust }}$ ) were correlated with each other. Expanse was also correlated with aggregate 


\begin{tabular}{lllllll} 
same & \multicolumn{6}{l}{ different (prey/predator) } \\
& $+/ 0$ & $0 /+$ & $+/-$ & $-/+$ & $-/ 0$ & $0 /-$ \\
\hline $35 / 48$ & 5 & 5 & 0 & 2 & 0 & 1
\end{tabular}

Table 23: Comparison of prey and predator linear regression models, with respect to the relationship between individual properties and aggregate properties. Numbers in the first column (same) indicate the number of times the prey and predator relationships were the same. Subsequent column numbers indicate the number of times a type of relationship was seen in the prey scenario and a different type of relationship was seen in the predator scenario. The table header indicates which was the prey relationship and which was the predator relationship (prey/predator). A ' 0 ' represents no relationship, a ' + ' represents directly related and a '-' represents inversely related.

$\begin{array}{lllllllll} & v_{\text {simp }} & \exp & p & n_{\text {groups }} & n_{\text {strag }} & n_{\text {clust }} & s_{\text {agg }} & t \theta_{\text {agg }} \\ v_{\text {simp }} & 1.00 & 0.81 & 0.11 & 0.30 & 0.47 & 0.45 & -0.10 & -0.08 \\ \exp & 0.81 & 1.00 & 0.20 & 0.68 & 0.81 & 0.80 & -0.18 & -0.11 \\ p & 0.11 & 0.20 & 1.00 & 0.21 & 0.24 & 0.24 & -0.44 & 0.55 \\ n_{\text {groups }} & 0.30 & 0.68 & 0.21 & 1.00 & 0.83 & 0.88 & -0.17 & -0.15 \\ n_{\text {strag }} & 0.47 & 0.81 & 0.24 & 0.83 & 1.00 & 0.99 & -0.26 & -0.13 \\ n_{\text {clust }} & 0.45 & 0.80 & 0.24 & 0.88 & 0.99 & 1.00 & -0.25 & -0.13 \\ s_{\text {agg }} & -0.10 & -0.18 & -0.44 & -0.17 & -0.26 & -0.25 & 1.00 & -0.64 \\ t \theta_{\text {agg }} & -0.08 & -0.11 & 0.55 & -0.15 & -0.13 & -0.13 & -0.64 & 1.00\end{array}$

Table 24: Correlation between aggregate measures- prey scenario. Correlation is significant at the 0.01 level (2-tailed), $n=2080$

cohesion measures $\left(n_{\text {groups }}, n_{\text {strag }}\right.$ and $n_{\text {clust }}$ ). Group angle was correlated with polarisation and group speed.

Aggregate measures that were correlated with each other had similar linear models (see Table 20 and Appendix B), so that, in most cases, when one aggregate measure was influenced by a particular combination of parameters, the other value was also influenced by these parameters. This did not mean that the aggregate measure was necessarily affected in the same way by the parameter. In some cases (e.g., group angle and group speed) factors caused an increase in one aggregate measure and a decrease in the other, resulting in a negative correlation between these measures.

For the predator scenario, with respect to correlation (correlation coefficient $r>0.5$, see Table 25), in addition to the expected correlations (simple volume and expanse, $n_{\text {strag }}$, numbers 
of groups and $n_{\text {clust }}$ ), $n_{\text {groups }}$ was correlated with both group size measures (simple volume and expanse). $n_{\text {strag }}$ and $n_{\text {clust }}$ was not correlated with either group size measure. Again, group angle was correlated with polarity and group size. Group angle was also negatively correlated with polarity.

$\begin{array}{lllllllll} & v_{\text {simp }} & \exp & p & n_{\text {groups }} & n_{\text {strag }} & n_{\text {clust }} & s_{\text {agg }} & t \theta_{\text {agg }} \\ v_{\text {simp }} & 1.00 & 0.71 & 0.14 & 0.52 & 0.19 & 0.27 & -0.01 & -0.26 \\ \exp & 0.71 & 1.00 & 0.19 & 0.55 & 0.16 & 0.26 & 0.00 & -0.36 \\ p & 0.14 & 0.19 & 1.00 & 0.29 & 0.35 & 0.36 & -0.73 & 0.58 \\ n_{\text {groups }} & 0.52 & 0.55 & 0.29 & 1.00 & 0.70 & 0.80 & -0.14 & -0.20 \\ n_{\text {strag }} & 0.19 & 0.16 & 0.35 & 0.70 & 1.00 & 0.99 & -0.31 & 0.00 \\ n_{\text {clust }} & 0.27 & 0.26 & 0.36 & 0.80 & 0.99 & 1.00 & -0.29 & -0.03 \\ s_{\text {agg }} & -0.01 & 0.00 & -0.73 & -0.14 & -0.31 & -0.29 & 1.00 & -0.55 \\ t \theta_{\text {agg }} & -0.26 & -0.36 & 0.58 & -0.20 & 0.00 & -0.03 & -0.55 & 1.00\end{array}$

Table 25: Correlation between aggregate measures- predator scenario. Correlation is significant at the 0.01 level (2-tailed), $n=2080$

\subsubsection{Discussion}

Complexity of aggregate behaviour As predicted and supported by the multi-way ANOVA results, all aggregate parameters were dependent on multiple, interacting parameters. The relationship between the values of parameters and the values of an aggregate measure were not additive, because of interactions between model parameters. This supports the view of an aggregate as a complex system. Although only 2 scenarios were modelled, it is clear from this that, when modelling actual aggregates, as many model parameters as possible must be set based on experimentally determined values. This further supports the argument that particular aggregate scenarios must be modelled explicitly, rather than relying on general aggregate model results. This is particularly the case when detailed or specific predictions are needed (the multiway ANOVA results will be discussed in more detail under Comparison of prey and predator behaviour, below).

The linear regression models of both scenarios confirmed the importance of $w_{c}$ and $w_{y}$, providing further proof that centring and velocity matching are crucial for aggregate behaviour 
and also indicating that these behaviours influence multiple properties observed at an aggregatelevel. Both scenarios showed that $s_{\max }$ and, in most cases, $w_{s}$ also influence observed aggregatelevel properties. The influence of centring and $w_{v}$ on aggregate properties also suggests that animals may be able to vary their aggregate behaviour simply through coordinated variance of their cognitive weight values. The influence of $s_{\max }$ on aggregate behaviours not obviously related to aggregate speed, (e.g., aggregate volume and aggregate polarity) suggests that if aggregating animals either choose to change their $s_{\max }$ in a given situation (due to conservation of energy considerations or a reaction to the appearance of a predator, for example), or are compelled to do so by environmental influences (e.g., a strong current, or tail wind), aggregatelevel behaviours may change in unanticipated ways. For example, individuals slowing down will not only mean that the aggregate as a whole slows down but also that the group becomes less aligned and smaller.

Comparison of prey and predator behaviour As predicted, models in the two visual field scenarios - the predator perceptual field and the prey perceptual field - behaved differently under the same parameter combinations. Across parameter combination maximums and minimums were comparable, which highlights the fact that, for these scenarios, some aggregate behaviours may be similar under some circumstances (i.e., for both aggregates, there may be times when their movement speed is very high and times when their movement angle is very low). However, the same combinations of aggregate behaviours do not have the same underlying causes, as represented by different individual properties. From an observational perspective, this means that a particular aggregate behaviour will not necessarily be seen in conjunction with the same other aggregate measures from one scenario to the next (e.g., one aggregate may have a high movement angle combined with a high aggregate volume whereas the other aggregate may more typically combine a high movement angle with a low aggregate volume).

This general conclusion was supported by the linear regression analysis of the two scenarios, which showed that in some cases the same factors had opposite effects on aggregate behaviour in the two scenarios. Specifically, with respect to the influence of the $w_{c}$ on the aggregate volume, an increasing $w_{c}$ decreased aggregate volume for the prey scenario (as was predicted) 
but increased it for the predator scenarios. The linear regression also revealed that although $t \theta_{\max }$ of individuals played a minimal a role in the behaviour of the prey aggregate, it played a large role in the behaviour of the predator aggregate. These differences explain why, as predicted in Section 6.3, the naïve predictions for the predator scenario were less accurate.

The multi-way ANOVA results highlighted the complexity of the two models. As can be seen from Table 19, for all aggregate measures there were numerous permutations of interacting factors. For example, in the case of $s_{\max }$ in the predator scenario, there were 4 unique factor interactions (interactions involving different combinations of factors), of varying sizes (3, 4 and 5). Thus, values for $s_{\max }$ could be predicted if the factors space pref $, w_{c}, w_{y}, s_{\max }$ and $t \theta_{\max }$ were known, but values could also be predicted if space $_{\text {pref }}, w_{c}, w_{s}$ and $t \theta_{\max }$ were known. As can be seen from Table 19, there were fewer unique interactions for the prey scenario (22 compared to 25 , across all aggregate measures), but these interactions tended to involve more factors (there were only one case with a smaller than 4-way interactions for aggregate measures, compared with 6 for the predator scenario). From this it might be argued that the prey scenario was a more complex scenario, in the sense that, for most aggregate measures, there were only high-level interactions between factors. However, the fact that there fewer interaction combinations exists argue that this system is also more stable than the predator scenario, because in the predator scenario more factors, over all, influence the behaviour of the aggregate. The argument that the prey scenario is more stable is also supported by the results of Table 18, which shows that the prey scenario is more cohesive.

Despite these differences, similarities across the two scenarios allow for some more general conclusions to be drawn about aggregates. For both scenarios, although there was variation in the size of the influence, all aggregate measures were strongly influenced by three model parameters: two cognitive parameters (centring and vector alignment) and one movement parameter (the $s_{\max }$ of the agents). This finding extends the research of Viscido et al. (2007), who found that all social forces except levels of randomness affected aggregate behaviour. Viscido et al. did not provide information on the extent and precise nature of these influences, but based on these model results, centring behaviours and vector alignment behaviours would be most influential, in combination with $s_{\max }$. Other cognitive parameters- space $_{\text {pref }}$ - and some physical 
parameters- $t \theta_{\text {max }}$ - had some effect on aggregate behaviour, but this effect was relatively low for most parameters.

Based on the correlations between aggregate behaviours model results, prey aggregates are expected to be either small and cohesive, or large and non-cohesive (with more groups and stragglers and, by extension, clusters). They are expected to be either aligned and moving in a straight line, or unaligned with more frequent turns. The speed of the aggregate is independent of these aggregate properties. In the case of the predator scenario, small aggregates will also have fewer groups, but not necessarily fewer stragglers (and by extension, clusters). Aligned groups are expected to move quickly, with fewer turns. Unaligned predator aggregates are expected to move slowly.

Necessary and sufficient cognitive abilities for aggregate cohesion The results of this analysis showed that when any one of $w_{c}, w_{v}$ or $w_{s}$ was 0 there was at least one run where $n_{c l u s t}$ remained 1 at $t=100$. This indicated that none of these behaviours by themselves are strictly necessary for group cohesion, over the period of time considered, for the parameter combinations considered (see Table 18). Similarly, if both alignment and distance behaviours, or both centring and distance behaviours were lacking (corresponding weights equal 0 ), $n_{\text {clust }}$ was still 1 in some runs, indicating that $w_{v}$ alone being greater than 0 or $w_{c}$ alone being greater than 0 was sufficient, in some cases, to maintain group cohesion up to $t=100$. However, when both $w_{v}$ and $w_{c}$ were 0 , then $n_{\text {clust }}$ was always above 1 , regardless of the values of other model parameters.

Based on these results, at least one of the centring or alignment behaviours is necessary in order for aggregation to occur, but it is possible for either alignment alone or centring alone to be sufficient for cohesive aggregation, contrary to the prediction by Grégoire et al. (2003). Considering the results of the linear regression, this is because multiple parameters may contribute in the same way to the same aggregate-level property. For example, (see Table 20) in the prey scenario both a high $w_{c}$ and a small preferred spacing value contribute to a small aggregate volume. Thus if the effect of the missing behaviour can be effectively duplicated as a result of the values of one or more of the other agent parameters (e.g., in the absence of the 
centring behaviour, $w_{v}$ is high, preferred spacing is small, $w_{s}$ is low and $s_{\max }$ is low) the $n_{\text {clust }}$ will still remain low.

\subsection{Chapter summary}

This chapter reviewed findings relating to how the aggregate model behaviour changed when varying cognitive and movement parameters. Two broad scenarios were considered in this context: one a prey aggregate analogue and one a predator aggregate analogue. Overall findings showed that the prey and predator scenarios were significantly different from each other. Although maximum and minimum aggregate values were similar for the two scenarios, the scenarios did not behave similarly when compared across parameter combinations. Further analysis of the behaviour of the prey analogue as compared to the predator analogue revealed that the prey analogue behaved in a more consistent aggregating behaviour, with respect to expected influences of parameters and overall cohesiveness of the aggregate. Despite the

differences seen across these two scenarios however, the strength of the centring behaviour, the vector alignment behaviour and the $s_{\max }$ of individuals influenced all aggregate measures considered, underscoring the importance of these individual behaviours to aggregation. As well, the complexity of the results support the argument that an aggregate is a complex system, with aggregate-level behaviours connected to individual properties in ways that are difficult to predict in advance of exploring the behaviour using techniques like IBSEM models. 


\section{Chapter 7}

\section{Conclusions and future work}

\subsection{Species-specific modelling with the PCA framework: A sum- mary of thesis contributions}

At the core of many aggregate models are the concepts of attractive and repulsive forces, which are analogies for physical processes taking place inside aggregating animals. As was discussed in Chapters 2 and 3, a major argument of this thesis is that this presents problems for aggregate researchers who are trying to make species-specific models, because the concepts of simple attractive and repulsive forces cannot easily be matched with the biological properties of individual animals. This, in turn, does not allow for verification of models using experimental data, particularly with respect to predictions concerning novel environmental circumstances. Thus, although theoretical aggregate models created with the AR framework may increase the level of understanding of basic aggregation concepts and requirements for aggregation, these models will have difficulty explaining how individuals from particular species interact to form aggregates, or why these aggregates have group-level properties that differ from the aggregates of other species. In order to create species-specific models, researchers need to incorporate properties that clearly differentiate animals with respect to how they are aggregating.

In Chapter 3 it was argued that these properties are perceptual properties (focusing on perceptions used to aggregate), cognitive properties (focusing on algorithms used to adjust position and movement relative to other individuals) and movement abilities (which constrain 
the ability of the animal to carry out its aggregating algorithms). To create concrete models that incorporate these properties, researchers can use the PCA framework, which was presented in Chapter 3. This framework incorporates some aspects of the attraction and repulsion framework but extends the abilities of this framework by reframing it into terms that are biologically based (e.g., attraction and repulsion forces are reframed as cognitive algorithms) and by increasing the detail of the representation (e.g., generic information fields are replaced by experimentally derived perceptual fields). The PCA framework makes this transition by making reference to the information processing paradigm of cognitive science.

As was stated in Chapter 3, in order for IBSEMs to be fully accepted as an animal aggregate modelling tool, researchers must create models that are sufficiently constrained to match the aggregate of interest rather than to animal aggregation in a more general sense. Using the PCA framework, researchers can now create species-specific IBSEM models that are informed by experimental data, by basing the properties of the individual agents on real properties, using the model to make predictions, and validating the model by comparing the behaviour of the model with the behaviour of the actual aggregate in question. Models created with this framework can be clearly compared with each other, so differences and similarities in aggregate can be highlighted with respect to perception, cognition and action.

To support these claims, Chapter 4 demonstrated that it was possible to take the general PCA framework, and use it to construct a species-specific aggregate model. Specifically, Chapter 4 demonstrated how three-dimensional position data and physical structure data could be collected, and used to create the species-specific and situation-specific Danio rerio model. Chapter 4 further showed that even though the model used only information about individuals to generate aggregate behaviour, it was able to predict aggregate properties. With respect to the behaviour of the Danio rerio aggregate, it was predicted that the aggregate volume, aggregate speed and aggregate movement of the 18 Danio rerio, in an aquarium environment, would oscillate over time, gradually increasing and then decreasing. This model behaviour was also seen in the actual aggregate, providing support for the validity of the model. In contrast to this, a simple AR model failed to make accurate predictions of aggregate behaviour, both qualitatively and quantitatively. More broadly speaking, Chapter 4 demonstrated the feasibility of creating aggregate models 
that are based on species-specific experimental data and incorporate multiple species-specific characteristics at the level of the individual, resulting in models with the ability to predict the qualitative and quantitative behaviour of species-specific aggregates.

Chapters 5 and 6 returned to more theoretical models, with the intent of exploring the significance and interaction of the components of the PCA framework. Chapter 5 investigated the information requirements of individuals in aggregates and how these information requirements were mediated by the shape of the perceptual field. The theoretical model presented at the beginning of the chapter established that relatively low amounts of information were required to aggregate, if this information was randomly sampled. A commonly used aggregation algorithm, the nearest neighbour algorithm, was shown to be less stable than this random algorithm, requiring substantially more information to generate even marginally stable aggregates. It was also determined that an aggregate can maintain cohesion even half of its individuals are missing information about the aggregate from moment to moment.

The remainder of Chapter 5 used these more general findings, in conjunction with models that varied the perceptual field shapes of agents, to explain why some perceptual field shapes allowed for aggregate cohesion while other perceptual field shapes did not promote aggregate cohesion. This work challenges the view that the shape of the perceptual field does not significantly influence aggregate behaviour. It showed that aggregates with agents that have different perceptual field shapes do not behave similarly, and that it is the shape, not the volume of the field that determines the stability of the aggregate. Equally importantly, however, every perceptual field shape had some model settings that allowed aggregation. This showed that perceptual field shape interac ts with other parameters and that an understanding of aggregate behaviour requires a specific consideration of the perceptual abilities of the aggregating species in question.

Chapter 6 completed the theoretical part of the thesis by examining the interaction between perceptual, cognitive and movement properties, and determining how these properties of individuals interact to influence aggregate-level properties. In general, every aggregate property that was measured was strongly influenced by the cognitive algorithms determining proximity and alignment, in conjunction with the maximum speed of individuals. In addition, the 
characterisation of aggregates as complex systems was strongly supported by the results of the multi-way analysis, which showed that there are numerous multi-way interactions between the properties of individuals that determine aggregate behaviours; predicting the particular aggregate-level behaviours of the model required knowledge of the specific values associated with each of these parameters in most cases. This finding further supports the general argument of the thesis that the properties of individuals in aggregate models must be set empirically and that generic aggregate models cannot easily be used to make predictions of species-specific aggregate behaviour.

\subsection{Limitations of current work and future research}

This thesis aims to motivate research into species-specific aggregation behaviours using the PCA framework. As such there are a variety of possibilities for future research arising out of Chapters 4, 5 and 6. Each of these chapters will be considered in turn with respect to limitations of the current work and future research possibilities.

\subsubsection{Chapter 4}

The Danio rerio model presented in Chapter 4 was intended to serve as a case study, demonstrating that it was possible to use the PCA framework to create a species-specific model. The model itself aimed to replicate the behaviour of a very specific aggregate- that of the 18 Danio rerio observed swimming in the constrained environment of a laboratory aquarium. As a result, this model was not intended to be a general model of Danio rerio aggregation. However, it would be possible to extend this model to create a more general model using several strategies. The first would be to collect additional data to confirm that the results gathered in this model are generalisable to the larger Danio rerio population. The second would be to study Danio rerio movement in a more naturalised setting (e.g., larger, rounded tank with dark non-reflective walls) and either confirm the generalisability of the Danio rerio data gathered in the aquarium context, or reset the model parameters based on this new, more naturalised data. 
The case study also demonstrated the challenges associated with setting model parameters using experimental data. In particular, it is currently difficult to gather data on the cognitive parameters and algorithms actually used by aggregating species. As was shown in Chapter 4, there are strategies available for creating models in the absence of this data, but more detailed and accurate data in this area could increase the accuracy and predictive power of the model. Given the status of the Danio rerio as a research animal, and the extensive research being undertaken to understand every aspect of its development, information on the cognitive mechanisms for the Danio rerio may eventually become available and could be incorporated. The explicit representation of cognition in the PCA framework, which forces researchers to confront cognitive questions like how aggregating animals resolve the binding problem and the frame problem may serve to direct this research.

The values for the perceptual abilities of animals are more easily determined experimentally, but there is still potential for developing research methods in this area. In this model, perceptual abilities were largely estimated based on prior research and physical evidence, and perceptual resolution was also estimated. However, there is the possibility of developing a research methodology to experimentally determine the perceptual range and resolution of aggregating animals. This methodology could then be applied to different species and used, in conjunction with aggregate models to increase an understanding of the role of perception in aggregation.

From a broader perspective, the intent of this chapter was to demonstrate that species-specific models that take into account individual properties will allow for the creation of predictive, species-specific models. It is hoped, therefore, that future research will involve creating new species-specific models. These models will be able to explore the behaviour of these aggregating species in varying environmental circumstances and predict behaviour in novel ones.

\subsubsection{Chapter 5}

Chapter 5 first presented a theoretical exploration of the information requirements for aggregation. Although it was determined that an individual's observation of one random conspecific can lead to aggregation, further research could be done in order to determine the relevance of 
the random component of the sample, and how this contributes to aggregation (in particular in contrast to the nearest neighbour algorithm). Determining the key difference between these two algorithms is particularly relevant because of the popularity of the nearest neighbour algorithm, and a general assumption by researchers that animals use the nearest neighbour algorithm to aggregate.

In addition, there are more general questions about necessary and sufficient quantity and quality of information, and the extent to which that information influences the behaviour of the model. Preliminary models that consider how weighting information received, relative to information about an individual's current movement, suggest that weighting by an individual can significantly influence aggregate properties, and might be used as a simple mechanism to alter aggregate properties over time. However, further investigation is required to understand how aggregate behaviour is influenced by the choice of the individual to attend more to its own movement vs. the movement of others.

The second part of Chapter 5 explored five simple perceptual field scenarios. Because the goal of the chapter was to compare perceptual fields which were similar, fields were chosen that may or may not have been representative of the perceptual fields of actual aggregating animals. Having established that perceptual field shape is relevant to aggregate behaviour, a next step in this research would be to create models with empirically derived perceptual field shapes, comparing the behaviours of these models. Agents in these models might also have perceptual fields representing multiple perceptions. Moving in a different direction, it would also be possible to investigate the influence of perceptual resolution on aggregate behaviour by increasing and decreasing the error equation associated with the perceptual field and determining how this influences aggregate measures. Additionally, it would be interesting to consider how perceptual fields influence the interaction of aggregates in the context of a more complex environment - for example, when they must avoid other objects in the environment (e.g., obstacles and predators) and when they are attracted to the presence of other objects (e.g., food) in addition to being repulsed by and attracted to each other. 


\subsubsection{Chapter 6}

Chapter 6 showcased the aggregate as a complex system. Although Chapter 6 demonstrated some possibilities for analysis of aggregate behaviour (multi-way ANOVA, linear regression), the results suggested that there is significant potential for additional analysis in this area. In particular, a more fine grained choice of parameter values, and the selection of more than two parameter values per parameter may lead to a more accurate linear regression model. As well, since the linear regression had fairly high error terms, it may be possible to investigate other forms of non-linear equation modelling in order to better predict the behaviour of the aggregate system. With respect to moving towards biologically relevant conclusions, the PCA framework makes it possible to connect these more general results to specific aggregates by matching the values of parameters relating to the perceptual fields, cognitive algorithms and movement algorithms of the agent with experimentally derived values. As well, two simple analogies (prey and predator) were considered. By increasing the range of values considered and by matching values to larger categories of aggregating species, it may be possible to reach some generalisable, but biologically plausible conclusions about different categories of aggregation seen in nature.

\subsection{Moving towards an understanding of species-specific aggrega- tion}

Aggregation is not an automatic or uniform phenomenon. Species aggregate in species-specific ways, driven by species-specific mechanisms. To capture this diversity of behaviour and begin to move away from simply understanding the basis of aggregation, aggregates need to be modelled in ways that pay attention to the unique characteristics and aggregating mechanisms of the individuals within the aggregate. The mechanisms of aggregation involve the perceptions of the animal, the cognition of the animal and the movement of the animal. Each of these aspects of the animal are connected in a perception, cognition, action cycle that is characterised by a flow of information from the environment, captured by the perceptions of the animal, processed 
by the cognition of the animal and used to determine the next movements of the animal, all in order to keep the animal moving with its conspecifics in an aggregating manner.

The prevalence and diversity of aggregation, coupled with its scale and complexity make for a rich and stimulating area of investigation. This thesis is intended to move researchers in the direction of investigating, in detail, the behaviour of specific aggregating species. Ultimately, the goal of this research direction might be several-fold: to understand how differing individual, biologically plausible, characteristics can lead to different types of aggregation, to study and characterise the different types of aggregation observed in nature, to catalogue, compare and contrast the behaviours of different types of aggregates and to study the specific mechanisms (perceptual, cognitive and movement) that aggregating individuals use, both within and across species. IBSEMS, coupled with the PCA framework- an information processing, perception focused framework that takes into account the perceptions, cognition and actions of individuals within the aggregate- are an effective new tool for exploration in this avenue of research. 


\section{References}

Adioui, M., Arino, O., Smith, W. V., \& Treuil, J. P. (2003). A mathematical analysis of a fish school model. Journal of differential equations, 188, 406-446.

Adioui, M., Treuil, J.P., \& Arino, O. (2003). Alignment in a fish school: A mixed LagrangianEulerian approach. Ecological modelling, 167, 19-32.

Aldana, M. \& Huepe, C. (2003). Phase Transitions in Self-Driven Many-Particle Systems and Related Non-Equilibrium Models: A Network Approach. Journal of Statistical Physics, 112(1/2), 135-153.

Aoki, I. (1982). A simulation study on the schooling mechanism in fish. Bulletin of the Japanese society of scientific fisheries, 48, 1081-1088.

Aoki, I., Inagaki T., \& Van Long, L. (1986). Measurements of the three-dimensional structure of free-swimming pelagic fish schools in a natural environment. Bulletin of the Japanese society of scientific fisheries, 52, 2069-2077.

Axelsen B. E., Anker-Nilssen, T., Fossum, P., Kvamme, C., \& Nøttestad, L. (2001). Pretty patterns, but a simple strategy: Predator-prey interactions between juvenile herring and atlantic puffins observed with multi-beam sonar. Canadian Journal of Zoology, 79, 15861596.

Ballerini, M., Cabibbo, N., Candelier, R., Cavagna, A., Cisbani, E., Giardina, I., Lecomte, V., Orlandi, A., Parisi, G., Procaccini, A., Viale, M., \& Zdravkovic, V. (2008). Interaction ruling animal collective behavior depends on topological rather than metric distance: Evidence from a field study. Proceedings of the National Academy of Sciences, 105, 1232-1237.

Ballerini, M., Cabibbo, N., Candelier, R., Cavagna, A., Cisbani, E., Giardina, I., Orlandi, A., Parisi, G., Procaccini, A., Viale, M., \& Zdravkovic, V. (2008). An empirical study of large, naturally occurring starling flocks: a benchmark in collective animal behaviour. Animal behaviour.

Bel'kovich, V. M. (1991). Herd structure, hunting and play: Bottlenose dolphins in the black sea. In K. Pryor \& K. S. Norris (Eds.), Dolphin societies: Discoveries and puzzles (pp. 17-76). Berkeley: University of California Press.

Bond, A. H., \& Gasser, L. (1988). Readings in distributed artificial intelligence. San Mateo, CA: Morgan Kaufmann.

Bossomaier, T. R. J., \& Green, D. G. (2000). Introduction. In T. R. J. Bossomaier \& D. G. Green (Eds.), Complex systems (p. 2). Cambridge, United Kingdom: Cambridge University Press. 
Breder, C. M. (1951). Studies on the structure of the fish school. Bulletin of the American museum of natural history, 98, article 1, 5-27.

Breder, C. M. (1954). Equations descriptive of fish schools and other animal aggregations. Ecology, 35, 361-370.

Breder, C. M. (1976). Fish schools as operational structures. U.S. fishery bulletin, 74, 471-502.

Brogan, D.C. \& Hodgins, J. K. (1997). Group Behaviors for Systems with Significant Dynamics. Autonomous Robots, 4(1), 137-153.

Bryson-Richardson, R., Berger, S., Schilling, T., Hall, T., Cole, N., Gibson, A., Sharpe, J., \& Currie, P. (2007). FishNet: An online database of zebrafish anatomy. BMC Biology, 5:34.

Buhl, J., Sumpter, D.J., Couzin, I.D., Hale, J., Despland, E, Miller, E \& Simpson, S.J. (2006). From disorder to order in marching locusts. Science, 312, 1402-1406.

Buskey, E. J. (2000). The role of vision in the aggregative behavior of the mysid Mysidium columbiae. Marine Biology, 137, 257-265.

Camazine, S., Deneubourg, J. L., Franks, N., Sneyd, J., Bonabeau, E., \& Theraulaz G. (2003). Self-organization in biological systems. Princeton, New Jersey: Princeton University Press.

Caraco, T., \& Bayham, M. C. (1982). Some geometric aspects of house sparrow flocks. Animal behavior, 30, 990-996.

Carbone, C., Thompson, W. A., Zadorina, L., \& Rowcliffe, J. M. (2003). Competition, predation risk and patterns of flock expansion in barnacle geese (Branta leucopsis). Journal of zoology, London, 259, 301-308.

Couzin, I. D., Krause, J., James, R., Ruxton, G. D., \& Franks, N. R. (2002). Collective memory and spatial sorting in animal groups. Journal of theoretical biology, 218, 1-11.

Couzin, I. D., Krause, J., Franks, N. R., \& Levin, S. A. (2005). Effective leadership and decisionmaking in animal groups on the move. Nature, 433, 513-516.

Czirók, A., Stanley, H. E. , \&Vicsek, T. (1997). Spontaneously ordered motion of self-propelled particles. Physica A, 30, 1375-1385.

Czirók, A., \& Vicsek, T. (2000). Collective behavior of interacting self-propelled particles. Physica $A, 281,17-29$.

Dagorn, L., \& Fréon, P. (1999). Tropical tuna associated with floating objects: a simulation study of the meeting point hypothesis. Canadian Journal of Fisheries and Aquatic Sciences, 56(6),984-993.

Dennett, D. (1984). Cognitive wheels: The frame problem of A.I. In C. Hookway (Ed.), Minds, Machines, and Evolution (pp. 129-151). New York: Cambridge University Press.

Edelstein-Keshet, L. (2001). Mathematical models of swarming and social aggregation. The 2001 International Symposium on Nonlinear Theory and its Applications (pp. 1-7). Miyagi, Japan.

Fancy, S. G. (1983). Movements and activity budgets of Caribou near oil drilling sites in the Sagavanirktok River floodplain, Alaska. Arctic, 38(2), 193-197.

Flierl, G., Grünbaum, D., Levin, S., \& Olson, D. (1999). From individuals to aggregations: The interplay between behavior and physics. Journal of theoretical biology, 196, 397-454. 
Frazer, N., \& Mercado, E. III (2000). A sonar model for humpback whale song. IEEE journal of oceanic engineering, 25(1), 160-182.

Gibson, J. J. (1977). The theory of affordances. In R. Shaw \& J. Bransford (Eds.), Perceiving, acting, and knowing: Toward an ecological psychology (pp. 67-82). Hillsdale, NJ: Lawrence Erlbaum

Godin, J.-G. J., Alfieri, M. S., Hoare, D. J., \& Sadowski, J. A. (2003). Conspecific familiarity and shoaling preferences in a wild guppy population. Canadian journal of zoology, 81, 1899-1904.

Graham, W. M., Pagès, F., \& Hamner, W. M. (2001). A physical context for gelatinous zooplankton aggregations: A review. Hydrobiologia, 451, 199-212.

Gerlotto, F., Bertrand, S., Bez, N. \& Gutiérrez, M. (2006). Waves of agitation inside anchovy schools: a way totransmit information and facilitate fast morphological and structural changes in response to predation, as observed with multibeam sonar. ICES Journal of Marine Science, 63, 1405-1417.

Gerlotto, F., \& Paramo, J. (2003). The three-dimensional morphology and internal structure of clupeid schools as observed using vertical scanning multibeam sonar. Aquatic living resources, 16, 113-122.

Greene, C. H., \& Wiebe, P. H. (1997). Acoustic visualization of three-dimensional animal aggregates in the ocean. In J. K. Parrish \& W. M. Hamner (Eds.), Animal groups in three dimensions (pp. 61-67). Cambridge: Cambridge University Press.

Grégoire, G., Chaté, H., \& Tu, Y. H. (2003). Moving and staying together without a leader. Physica D, 181, 157-170.

Grünbaum, D. (1999). Advection-diffusion equations for generalized tactic searching behaviors. Journal of Mathematical Biology, 38, 169-194.

Grünbaum, D., \& Okubo, A. (1994). Modelling social animal aggregations. In S. Levin (Ed.), Frontiers of theoretical biology (pp. 296-325). (Lecture notes in biomathematics v. 100). New York: Springer Verlag.

Grünbaum, D., Viscido, S., Parrish, J. K. (2005). Extracting interactive control algorithms from group dynamics of schooling fish. Cooperative control, 309, 103-117.

Gueron, S., \& Levin, S. A. (1993). Self-organization of front patterns in large wildebeest herds. Journal of theoretical biology, 165, 541-552.

Hamner, W. M., \& Hamner, P. P. (2000). Behavior of Antarctic krill (Euphausia superba): Schooling, foraging and antipredatory behavior. Canadian journal of fisheries and aquatic sciences, 57, 192-202.

Harrington, F. H., \& Veitch, A. M. (1991). Short-term impacts of low-level jet fighter training on caribou in Labrador. Arctic, 44(4), 318-327.

Hartley, R., \& Zisserman, A. (2003). Multiple view geometry in computer vision. New York: Cambridge University Press.

Helbing, D., Farkas, I. J., Molnár, P., \& Vicsek, T. (2002). Simulation of pedestrian crowds in normal and evacuation situations. In M. Schreckenberg \& S. D. Sharma (Eds.), Pedestrian and evacuation dynamics (pp. 21-58). New York: Springer-Verlag. 
Helbing, D., \& Molnár, P. (1995). Social force model for pedestrian dynamics. Physical Review E, 51, 4282-4286.

Hemelrijk, C. K. (2003). Understanding social behaviour with the help of complexity science. Ethology, 108, 655-671.

Hemelrijk, C. K., \& Hildenbrandt, H. (2008). Self-organized shape and frontal density of fish schools. Ethology, 114, 245-254.

Hemmings, C. C. (1966). Olfaction and vision in fish schooling. Journal of experimental biology, $45,449-464$.

Heppner, F. (1997). Three-dimensional structure and dynamics of bird flocks. In J. K. Parrish \& W. M. Hamner (Eds.), Animal groups in three dimensions (pp. 68-89). Cambridge: Cambridge University Press.

Hoare, D.J., Couzin, I.D., Godin, J.G.J. \& Krause, J. (2004). Context-dependent group size choice in fish. Animal Behaviour, 67, 155-164.

Holyoak, K. J. (1999). Preface: Psychology. In R. A. Wilson and F. C. Keil (Eds.), MIT encyclopedia of the cognitive sciences (pp. xxoxix-xlix). Cambridge, MA: MIT Press.

Hummel, J. E., \& Biederman, I. (1992). Dynamic binding in a neural network for shape recognition. Psychological Review, 99, 480-517.

Huth, A., \& Wissel. C. (1992). The simulation of the movement of fish schools. Journal of theoretical biology, 156, 365-385.

Ikawa, T., \& Okabe, H. (1997). Three-dimensional measurements of swarming mosquitoes: A probabilistic model, measuring system, and example results. In J. K. Parrish \& W. M. Hamner (Eds.), Animal groups in three dimensions (pp. 90-104). Cambridge: Cambridge University Press.

Jaffe, J. S. (1997). Methods for three-dimensional sensing of animals. In J. K. Parrish \& W. M. Hamner (Eds.), Animal groups in three dimensions (pp. 17-35). Cambridge: Cambridge University Press.

Jeanson, R. , Rivault, C., Deneubourg, J.L., Blanco, S., Fournier, R., Jost, C. \& Theraulaz, G. (2005). Self-organised aggregation in cockroaches. Animal Behaviour, 69, 169-180.

Klein, J. (2002). Breve: A 3D simulation environment for the simulation of decentralized systems and artificial life. In R. Standish, M. A. Bedau, \& H. A. Abbass (Eds.), Proceedings of artificial life VIII, the 8th international conference on the simulation and synthesis of living systems (pp. 329-334). Cambridge, MA: MIT Press.

Krause, J., Godin, J.-G. J., \& Brown, D. (1996). Phenotypic variability within and between fish shoals. Ecology, 77, 1586-1591.

Krause, J., Hoare, D. J., Croft, D., Lawrence, J., Ward, A., Ruxton, G. D., Godin, J.-G. J., \& James, R. (2000). Fish shoal composition: Mechanisms and constraints. Proceedings of the royal society $B, 267,2011-2017$.

Krause, J., \& Ruxton, G. D. (2002). Living in groups. Oxford: Oxford University Press.

Krause, J., \& Tegeder, R. (1994). The mechanism of aggregation behaviour in fish shoals: Individuals minimize approach time to neighbours. Animal Behavior, 48, 353-359. 
Kunz, H., \& Hemelrijk, C. K. (2003). Artificial fish schools: Collective effects of school size, body size, and body form. Artificial life, 9, 237-253.

Levenson D. H., \& Schusterman R. J. (1999). Dark adaptation and visual sensitivity in shallow and deep-diving pinnipeds. Marine Mammal Science, 15, 1303-1313.

Lutscher, F. (2003) A model for speed adaptation of individuals and existence of weak solutions. European journal of applied mathematics, 14, 291-311.

Mach, R. \&Schweitzer, F. (2007). Modeling Vortex Swarming In Daphnia. Bulletin of Mathematical Biology, 69, 539-562.

Miller, N., \& Gerlai, R. (2007). Quantification of shoaling behaviour in zebrafish (Danio rerio). Behavioural brain research, 184(2), 157-166.

Miller, F. L., Jonkel, C. J., \& Tessier, G. D. (1972). Group cohesion and leadership response by barren ground caribou to man-made barriers. Arctic, 25(3), 193-202.

Miller, R. S., \& Stephen, W. J. D. (1966). Spatial relationships in flocks of sandhill cranes. Ecology, 47(2), 323-327.

Mogilner, A., Edelstein-Keshet, L., Bent, L., \& Spiros, A. (2003). Mutual interactions, potentials, and individual distance in a social aggregation. Journal of mathematical biology, 47, 353389.

Moiseff, A., \& Copeland, J. (2000) A new type of synchronized flashing in a north american firefly. Journal of insect behavior, 13, 597-612.

Moorman, S. J. (2001). Development of sensory systems in zebrafish (Danio rerio). ILAR Journal, 42(4), 292-298.

Neisser, U. (1999). Ecological psychology. In R. A. Wilson \& F. C. Keil (Eds.), MIT encyclopedia of the cognitive sciences (pp. 255-257). Cambridge, MA: MIT Press.

Okubo A. (1986). Dynamical aspects of animal grouping: swarms, schools, flocks, and herds. Advances in Biophysics, 22, 1-94.

Osborn, J. (1997) Analytical and digital photogrammetry. In J. K. Parrish \& W. M. Hamner (Eds.), Animal groups in three dimensions (pp. 36-60). Cambridge: Cambridge University Press.

Parr, A. E. (1927). A contribution to the theoretical analysis of the schooling behavior of fishes. Occasional papers of the Bingham Oceanography College, 1, 1-32.

Parrish, J. K., \& Edelstein-Keshet, L. (1999). Complexity, pattern, and evolutionary trade-offs in animal aggregation. Science, 284(5411), 99-101.

Parrish, J. K. \& Viscido, S. V. (2005). Traffic rules of fish schools: a review of agent-based approaches. In Hemelrijk, C. K. (ed.) Self-Organisation and the Evolution of Social Behaviour. Cambridge Univ. Press, Cambridge, UK.

Parrish, J. K., Viscido, S. V., \& Grünbaum, D. (2002). Self-organized fish schools: An examination of emergent properties. Biological bulletin, 202, 296-305.

Partridge, B. L., \& Pitcher, T. J. (1980). The sensory basis of fish schools: Relative roles of lateral line and vision. Journal of comparative physiology A, 135, 315-325. 
Pearson Scott Foresman (n.d.) [Anchovy]. Image donated to Wikimedia Foundation and released into the public domain by Pearson Scott Foresman. Retrieved 2008-08-15 from http://commons.wikimedia.org/wiki/Image:Anchovy_(PSF).png

Protasov, V. R. (1970). Vision and near orientation of fish. Jerusalem: Israel Program for Scientific Translation.

Reynolds, C. (1987). Flocks, herds and schools: A distributed behavioural model. Computer graphics, 21(4), 25-34.

Rivers, N. M., Butlin, R. K., \& Altringham, J. D. (2006). Autumn swarming behaviour of Natterer's bats in the UK: Population size, catchment area and dispersal. Biological conservation, 127(2), 215-226.

Rohani, P., Lewis, T. J., Grünbaum, D., \& Ruxton, G. D. (1997). Spatial self-organization in ecology: Pretty pictures or robust reality? Trends in ecology and evolution, 12, 70-74.

Schellinck, J., \& White, T. (2005). Use of Netlogo as a rapid prototyping tool for the creation of more rigorous spatially explicit individual-based biological models. In D. R. C. Hill, V. Barra, \& M. K. Traore Open International Conference on Modelling and Simulation-OICMS 2005 (pp. 241-248). Clermont-Ferrand: Blaise Pascal University.

Schneck, M. E., Haegerstrom-Portnoy, G., Lott, L. A., Brabyn, J. A., \& Gildengorin, G. (2004). Low contrast vision function predicts subsequent acuity loss in an aged population: The SKI study. Vision research, 44, 2317-2325.

Selous, E., (1931). Thought-transference (or what?) in birds. Cited in Heppner (1997). London: Constable \& Co.

Singer, W. (1996). Neuronal synchronization: A solution to the binding problem. In R. Llinás \& P. S. Churchland (Eds.), The mind-brain continuum: sensory processes (pp. 101-130). Cambridge, MA: MIT Press.

Soria, M., Bahri, T., \& Gerlotto, F. (2003). Effect of external factors (environment and survey vessel) on fish school characteristics observed by echosounder and multibeam sonar in the Mediterranean Sea. Aquatic living resources, 16(3), 145-157.

Spieler, M. (2003). Risk of predation affects aggregation size: A study with tadpoles of Phrynomantis microps (Anura: Microhylidae). Animal behaviour, 65(1), 179-184.

Stöcker, S. (1999). Models for tuna school formation. Mathematical Biosciences, 156, 157-190.

Toner, J., \& Tu, Y. (1999). Flocks, herds and schools: A quantitative theory of flocking. Physical Review E, 58, 4828-4858.

Tu, Y. (2000). Phases and phase transitions in flocking systems. Physica A, 281, 30-40.

Turner, M. G., Wu, Y., Romme, W. H., \& Wallace, L. L. (1993). A landscape simulation model of winter foraging by large ungulates. Ecological modelling, 69, 163-184.

Vicsek, T., Czirók, A., Ben-Jacob, E. \& Cohen I. (1995). Novel type of phase transition in a system of self-driven particles. Physical Review Letters, 75, 1226.

Van Trump, W.J., McHenry, M.J. (2008). Lateral line morphology and sensitivity in zebrafish larvae (Danio rerio). Journal of Experimental Biology, 211(13), 2105-15. 
Viscido, S. V. (2002, April 3). Fish schooling experiments. Originally retrieved 2005-02-13 from http://depts.washington.edu/ birdfish/schooling/expts.shtml. Archived version retrieved on 2008-09-28 from http://web.archive.org/web/20050213024716/depts.washington.edu/ $\sim$ birdfish/schooling/expts.shtml

Viscido S. V., Miller, M., \& Wethey, D. S. (2002). The dilemma of the selfish herd: The search for a realistic movement rule. Journal of theoretical biology, 217(2), 183-194.

Viscido, S. V., Parrish, J. K., \& Grünbaum, D. (2004). Individual behavior and emergent properties of fish schools: A comparison of observation and theory. Marine ecology progress series, 273, 239-249.

Viscido, S. V., Parrish, J. K., \& Grünbaum, D. (2005). The effect of population size and number of influential neighbors on the emergent properties of fish schools. Ecological modelling, 183, 347-363.

Viscido, S.V., Parrish, J.K. \& Grünbaum, D. (2007). The relative importance of social and nonsocial factors in the formation and maintenance of fish schools. Ecological Modelling, 206, 153-165.

Vollmer, J., Vegh, A., Lange, C. \&Eckhardt, B. (2006). Vortex formation by active agents as a model for Daphnia swarming. Physical Review E., 73(6), 061924. Epub.

Warburton, K. \& Lazarus, J. (1991). Tendency-distance models of social cohesion in animal groups. Journal of Theoretical Biology, 150, 473-488.

Ward, C. R., Gobet, F., \& Kendall, G. (2001). Evolving collective behavior in an artificial ecology. Artificial life, 7(2), 191-209.

Whitfield, T.T., Granato, M., van-Eeden, F.J., Schach, U., Brand, M., Furutani-Seiki, M., Haffter, P., Hammerschmidt, M., Heisenberg, C.P., Jiang, Y.J., Kane, D.A., Kelsh, R.N., Mullins, M.C., Odenthal, J. \& Nüsslein-Volhard, C. (1996). Mutations affecting development of the zebrafish inner ear and lateralline. Development, 123, 241-254.

Wiegand, T., Revilla, E., \& Knauer, F. (2004). Dealing with uncertainty in spatially explicit population models. Biodiversity and conservation, 13, 53-78.

Zheng, M., Kashimori, Y., Hoshino, O., Fujita, K., \& Kambara, T. (2005). Behavior pattern (innate action) of individuals in fish schools generating efficient collective evasion from predation. Journal of theoretical biology, 235, 153-167. 


\section{Appendix A}

\section{Maximum, Minimum and Mean Values for all 16 Parameter Combinations of The Danio rerio Model}

This appendix presents model values for the PCAI and simple AR Danio rerio models. For each model, the maximum average, average and minimum average values of 5 aggregate measures, $v_{\text {simp }}$, exp, $p, s_{a g g}$ and $t \theta_{\text {agg }}$ (30 runs of 450 and 451 timesteps respectively, for the PCAI and simple AR model) are presented for each of the 16 parameter values considered (see Table 6 in Chapter 4 for the parameter values associated with each name). Values for number of clusters, number of groups and numbers of stragglers are not shown in these tables, because these values were always 1, 1 and 0 for all runs of all parameter combinations for both of these models. This was not necessarily the case, as can be seen by results presented in Chapter 5 . However, the parameter values chosen for these 16 runs for these two models did always result in a single cluster of agents at all times. 


$\begin{array}{ll}\text { name } & v_{\text {simp }} \\ 10111 & 8.11 \pm 3.00 \\ 10113 & 7.12 \times 10^{2} \pm 5.11 \times 10^{1} \\ 10133 & 6.89 \times 10^{2} \pm 5.72 \times 10^{1} \\ 10311 & 8.92 \times 10^{-2} \pm 8.29 \times 10^{-3} \\ 10313 & 8.22 \pm 3.37 \\ 10331 & 2.77 \times 10^{-1} \pm 2.77 \times 10^{-2} \\ 10333 & 7.38 \times 10^{1} \pm 3.82 \times 10^{1} \\ 6111 & 7.54 \pm 2.85 \\ 6113 & 1.61 \times 10^{2} \pm 1.24 \times 10^{1} \\ 6131 & 3.17 \times 10^{1} \pm 1.29 \times 10^{1} \\ 6133 & 1.59 \times 10^{2} \pm 1.39 \times 10^{1} \\ 6311 & 8.92 \times 10^{-2} \pm 8.29 \times 10^{-3} \\ 6313 & 7.87 \pm 3.37 \\ 6331 & 2.77 \times 10^{-1} \pm 2.77 \times 10^{-2} \\ 6333 & 2.68 \times 10^{1} \pm 1.15 \times 10^{1} \\ 10131 & 9.30 \times 10^{1} \pm 5.50 \times 10^{1}\end{array}$

\section{$\exp$}

$5.76 \times 10^{-1} \pm 5.43 \times 10^{-2}$

$2.90 \pm 2.58 \times 10^{-2}$

$2.90 \pm 2.42 \times 10^{-2}$

$1.82 \times 10^{-1} \pm 3.46 \times 10^{-3}$

$5.76 \times 10^{-1} \pm 5.64 \times 10^{-2}$

$2.13 \times 10^{-1} \pm 3.52 \times 10^{-3}$

$1.20 \pm 3.72 \times 10^{-1}$

$5.62 \times 10^{-1} \pm 6.01 \times 10^{-2}$

$1.73 \pm 2.82 \times 10^{-2}$

$9.21 \times 10^{-1} \pm 2.16 \times 10^{-1}$

$1.73 \pm 2.46 \times 10^{-2}$

$1.82 \times 10^{-1} \pm 3.46 \times 10^{-3}$

$5.63 \times 10^{-1} \pm 6.57 \times 10^{-2}$

$2.13 \times 10^{-1} \pm 3.52 \times 10^{-3}$

$8.74 \times 10^{-1} \pm 1.99 \times 10^{-1}$

$1.24 \pm 4.14 \times 10^{-1}$ $p$

$1.18 \times 10^{-1} \pm 7.47 \times 10^{-3}$

$2.04 \times 10^{-1} \pm 1.15 \times 10^{-2}$

$4.05 \times 10^{-1} \pm 2.51 \times 10^{-2}$

$1.71 \times 10^{-1} \pm 4.06 \times 10^{-3}$

$1.15 \times 10^{-1} \pm 8.64 \times 10^{-3}$

$4.84 \times 10^{-1} \pm 2.61 \times 10^{-2}$

$4.03 \times 10^{-1} \pm 7.32 \times 10^{-2}$

$1.14 \times 10^{-1} \pm 8.38 \times 10^{-3}$

$2.27 \times 10^{-1} \pm 1.46 \times 10^{-2}$

$4.34 \times 10^{-1} \pm 8.31 \times 10^{-2}$

$4.11 \times 10^{-1} \pm 3.41 \times 10^{-2}$

$1.71 \times 10^{-1} \pm 4.06 \times 10^{-3}$

$1.16 \times 10^{-1} \pm 8.91 \times 10^{-3}$

$4.84 \times 10^{-1} \pm 2.61 \times 10^{-2}$

$4.00 \times 10^{-1} \pm 6.88 \times 10^{-2}$

$4.57 \times 10^{-1} \pm 9.87 \times 10^{-2}$ $s_{a g g}$

$6.78 \times 10^{-1} \pm 3.38 \times 10^{-1}$

$1.76 \pm 1.66 \times 10^{-1}$

$2.77 \times 10^{-1} \pm 1.09 \times 10^{-1}$

$2.66 \pm 4.01 \times 10^{-3}$

$6.81 \times 10^{-1} \pm 3.69 \times 10^{-1}$

$2.15 \pm 1.56 \times 10^{-2}$

$2.69 \times 10^{-1} \pm 4.04 \times 10^{-1}$

$9.75 \times 10^{-1} \pm 4.29 \times 10^{-1}$

$1.59 \pm 1.58 \times 10^{-1}$

$2.02 \times 10^{-1} \pm 7.80 \times 10^{-2}$

$2.58 \times 10^{-1} \pm 8.83 \times 10^{-2}$

$2.66 \pm 4.01 \times 10^{-3}$

$1.26 \pm 4.05 \times 10^{-1}$

$2.15 \pm 1.56 \times 10^{-2}$

$2.34 \times 10^{-1} \pm 9.09 \times 10^{-2}$

$2.76 \times 10^{-1} \pm 4.06 \times 10^{-1}$ $t \theta_{\text {agg }}$

$5.06 \times 10^{-3} \pm 2.23 \times 10^{-3}$

$7.52 \times 10^{-3} \pm 4.62 \times 10^{-3}$

$2.49 \times 10^{-2} \pm 1.33 \times 10^{-2}$

$1.79 \times 10^{-3} \pm 1.01 \times 10^{-3}$

$5.89 \times 10^{-3} \pm 2.84 \times 10^{-3}$

$9.26 \times 10^{-3} \pm 5.19 \times 10^{-3}$

$5.02 \times 10^{-2} \pm 1.59 \times 10^{-1}$

$5.32 \times 10^{-3} \pm 3.09 \times 10^{-3}$

$7.92 \times 10^{-3} \pm 4.83 \times 10^{-3}$

$2.26 \times 10^{-2} \pm 1.11 \times 10^{-2}$

$2.81 \times 10^{-2} \pm 1.51 \times 10^{-2}$

$1.79 \times 10^{-3} \pm 1.01 \times 10^{-3}$

$6.86 \times 10^{-3} \pm 2.72 \times 10^{-3}$

$9.26 \times 10^{-3} \pm 5.19 \times 10^{-3}$

$2.67 \times 10^{-2} \pm 1.68 \times 10^{-2}$

$4.69 \times 10^{-2} \pm 1.60 \times 10^{-1}$

Table 26: Minimum values for Danio rerio model 


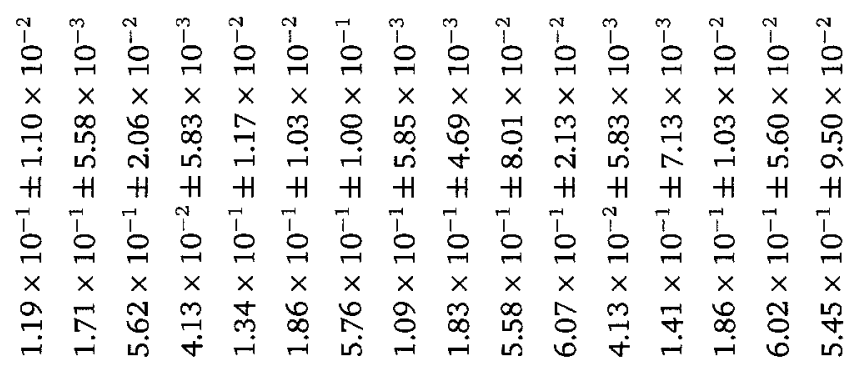

î

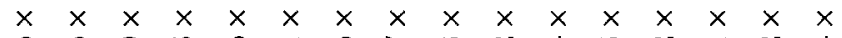
\&

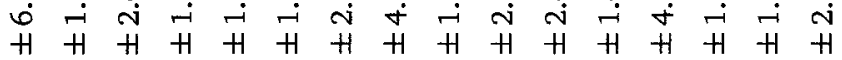
$\infty$

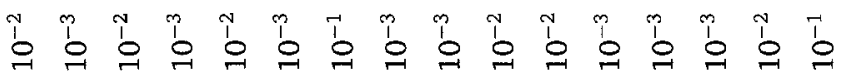

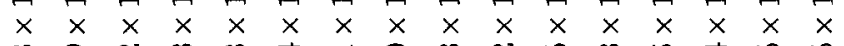
负

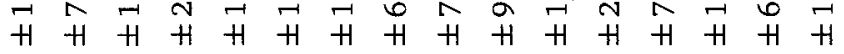

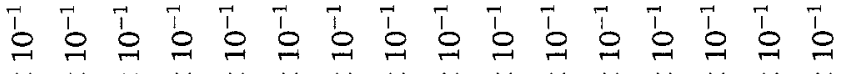

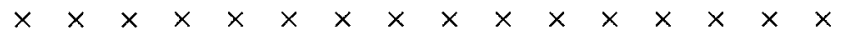

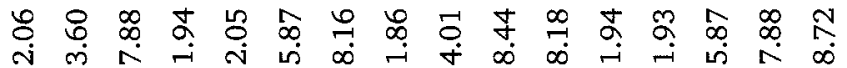

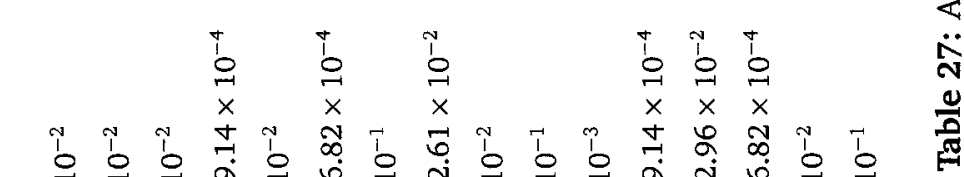
$\vec{x} \vec{x}+\vec{x}+\vec{x}+\vec{x} \vec{x} \vec{x}+1+H \vec{x} \vec{x}$

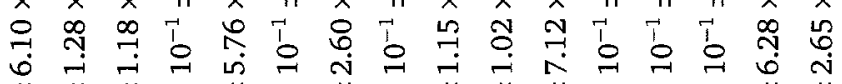

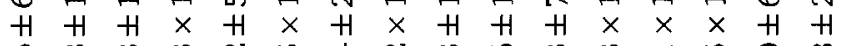

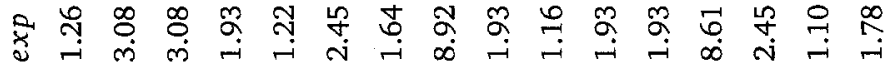

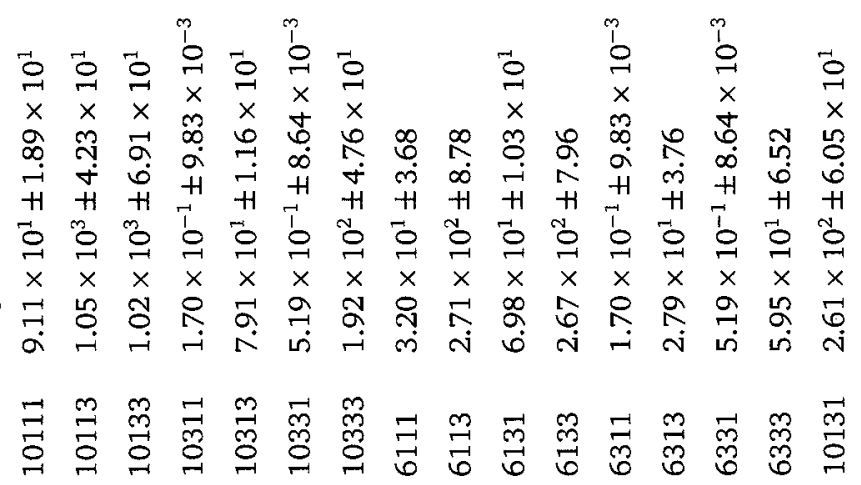




\begin{tabular}{|c|c|c|c|c|c|}
\hline name & $v_{\text {simp }}$ & $\exp$ & $p$ & $s_{\text {agg }}$ & $t \theta_{a g g}$ \\
\hline 10111 & $1.78 \times 10^{2} \pm 3.86 \times 10^{1}$ & $1.61 \pm 5.38 \times 10^{-2}$ & $6.81 \times 10^{-1} \pm 2.95 \times 10^{-1}$ & $2.65 \pm 2.73 \times 10^{-2}$ & $8.75 \times 10^{-1} \pm 4.53 \times 10^{-1}$ \\
\hline 10113 & $1.54 \times 10^{3} \pm 1.17 \times 10^{2}$ & $3.31 \pm 4.11 \times 10^{-2}$ & $6.85 \times 10^{-1} \pm 5.28 \times 10^{-2}$ & $2.65 \pm 1.36 \times 10^{-2}$ & $9.34 \times 10^{-1} \pm 4.02 \times 10^{-1}$ \\
\hline 10133 & $1.47 \times 10^{3} \pm 1.34 \times 10^{2}$ & $3.27 \pm 3.21 \times 10^{-2}$ & $45 \pm 6.03 \times 10^{-2}$ & $2.43 \pm 4.55 \times 10^{-2}$ & $2.46 \pm 2.65 \times 10^{-1}$ \\
\hline 10311 & $2.70 \times 10^{-1} \pm 1.77 \times 10^{-2}$ & $1.99 \times 10^{-1} \pm 1.40 \times 10^{-3}$ & $2.18 \times 10^{-1} \pm 6.62 \times 10^{-3}$ & $2.69 \pm 1.82 \times 10^{-3}$ & $1.73 \times 10^{-1} \pm 8.42 \times 10^{-2}$ \\
\hline 10313 & $1.58 \times 10^{2} \pm 2.93 \times 10^{1}$ & $1.55 \pm 6.32 \times 10^{-2}$ & $7.01 \times 10^{-1} \pm 3.25 \times 10^{-1}$ & $2.67 \pm 9.95 \times 10^{-3}$ & $9.20 \times 10^{-1} \pm 4.28 \times 10^{-1}$ \\
\hline 10331 & $9.41 \times 10^{-1} \pm 7.63 \times 10^{-2}$ & $2.79 \times 10^{-1} \pm 4.85 \times 10^{-3}$ & $6.60 \times 10^{-1} \pm 8.41 \times 10^{-3}$ & $2.38 \pm 2.35 \times 10^{-2}$ & $6.85 \times 10^{-1} \pm 1.51 \times 10^{-1}$ \\
\hline 10333 & $3.32 \times 10^{2} \pm 7.36 \times 10^{1}$ & $1.88 \pm 2.27 \times 10^{-1}$ & $1.44 \pm 2.08 \times 10^{-1}$ & $2.37 \pm 1.27 \times 10^{-1}$ & $2.47 \pm 3.31 \times 10^{-1}$ \\
\hline 6111 & $5.60 \times 10^{1} \pm 7.71$ & $1.05 \pm 3.76 \times 10^{-2}$ & $5.13 \times 10^{-1} \pm 2.76 \times 10^{-1}$ & $2.66 \pm 1.95 \times 10^{-2}$ & $7.18 \times 10^{-1} \pm 4.35 \times 10^{-1}$ \\
\hline 6113 & $4.65 \times 10^{2} \pm 6.08 \times 10^{1}$ & $2.20 \pm 6.26 \times 10^{-2}$ & $7.74 \times 10^{-1} \pm 7.15 \times 10^{-2}$ & $2.63 \pm 1.47 \times 10^{-2}$ & $1.01 \pm 3.77 \times 10^{-1}$ \\
\hline 6131 & $1.27 \times 10^{2} \pm 1.20 \times 10^{1}$ & $1.36 \pm 3.17 \times 10^{-2}$ & $1.50 \pm 5.70 \times 10^{-2}$ & $2.32 \pm 1.42 \times 10^{-1}$ & $2.53 \pm 3.21 \times 10^{-1}$ \\
\hline 6133 & $4.37 \times 10^{2} \pm 3.43 \times 10^{1}$ & $2.13 \pm 2.94 \times 10^{-2}$ & $1.47 \pm 6.26 \times 10^{-2}$ & $2.40 \pm 4.62 \times 10^{-2}$ & $2.63 \pm 2.31 \times 10^{-1}$ \\
\hline 6311 & $2.70 \times 10^{-1} \pm 1.77 \times 10^{-2}$ & $1.99 \times 10^{-1} \pm 1.40 \times 10^{-3}$ & $2.18 \times 10^{-1} \pm 6.62 \times 10^{-3}$ & $2.69 \pm 1.82 \times 10^{-3}$ & $1.73 \times 10^{-1} \pm 8.42 \times 10^{-2}$ \\
\hline 6313 & $5.24 \times 10^{1} \pm 8.74$ & $1.02 \pm 3.99 \times 10^{-2}$ & $3.94 \times 10^{-1} \pm 1.60 \times 10^{-1}$ & $2.68 \pm 7.47 \times 10^{-3}$ & $7.94 \times 10^{-1} \pm 3.96 \times 10^{-1}$ \\
\hline 6331 & $9.41 \times 10^{-1} \pm 7.63 \times 10^{-2}$ & $2.79 \times 10^{-1} \pm 4.85 \times 10^{-3}$ & $6.60 \times 10^{-1} \pm 8.41 \times 10^{-3}$ & $2.38 \pm 2.35 \times 10^{-2}$ & $6.85 \times 10^{-1} \pm 1.51 \times 10^{-1}$ \\
\hline 6333 & $1.08 \times 10^{2} \pm 8.77$ & $1.28 \pm 2.25 \times 10^{-2}$ & $1.45 \pm 6.40 \times 10^{-2}$ & $2.40 \pm 1.15 \times 10^{-1}$ & $2.51 \pm 3.29 \times 10^{-1}$ \\
\hline 10131 & $4.41 \times 10^{2} \pm 9.07 \times 10^{1}$ & $2.08 \pm 2.35 \times 10^{-1}$ & $1.45 \pm 2.02 \times 10^{-1}$ & $2.27 \pm 1.81 \times 10^{-1}$ & $2.43 \pm 2.75 \times 10^{-1}$ \\
\hline
\end{tabular}

Table 28: Maximum values for Danio rerio model 


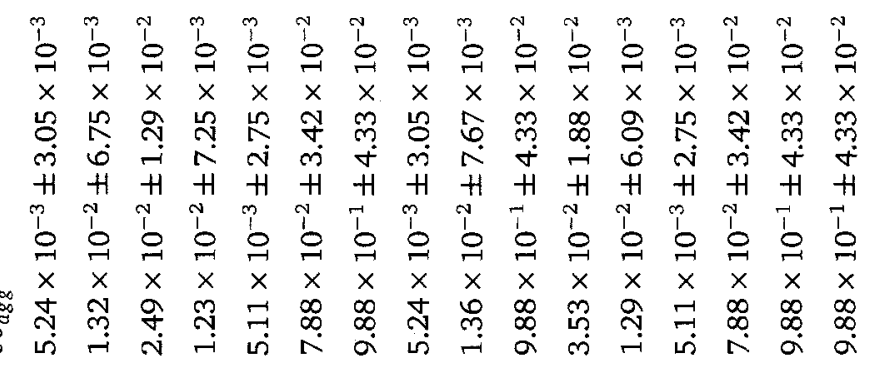

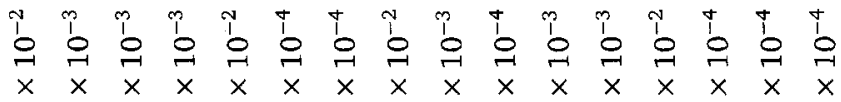

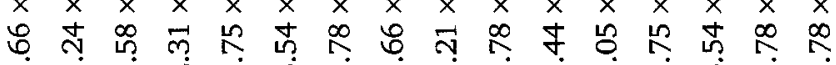
H $\vec{H} \dot{H}$ H

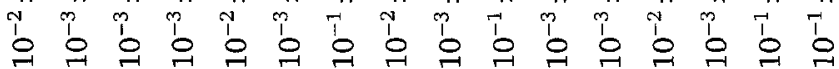

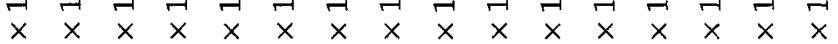
ڤ

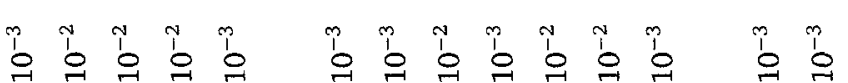

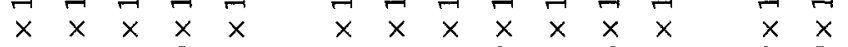

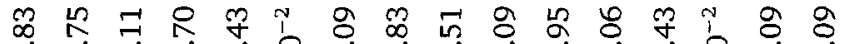

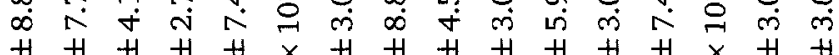

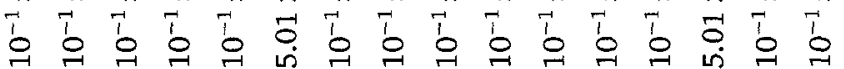

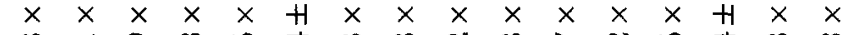

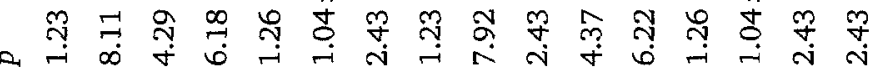

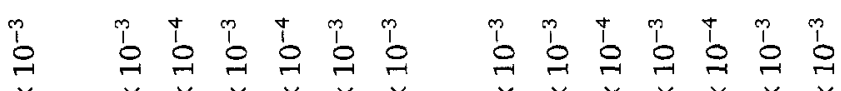

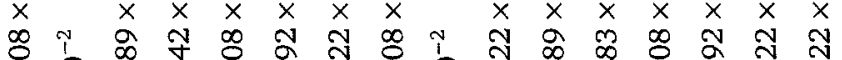

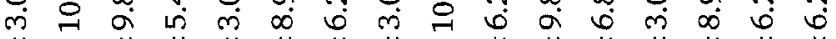

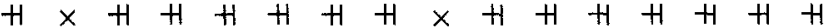
İ

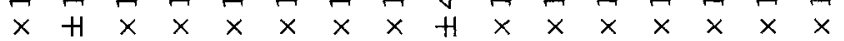

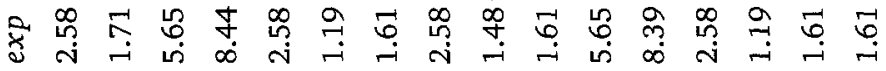

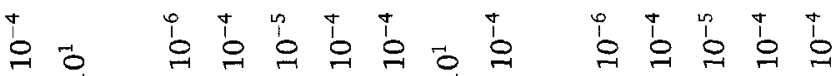

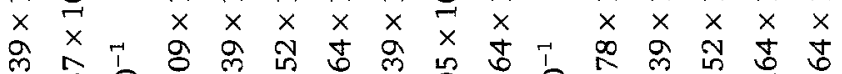

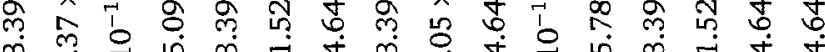

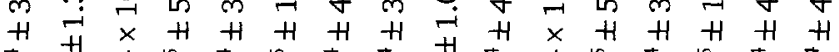

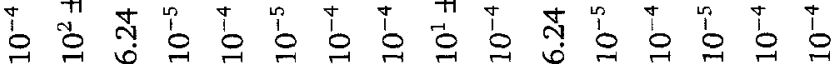

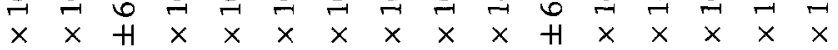

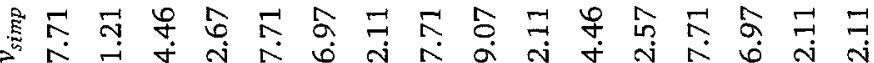

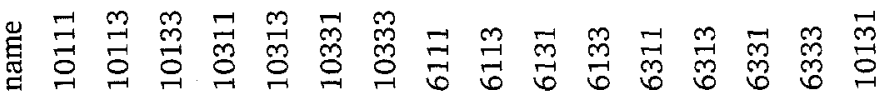




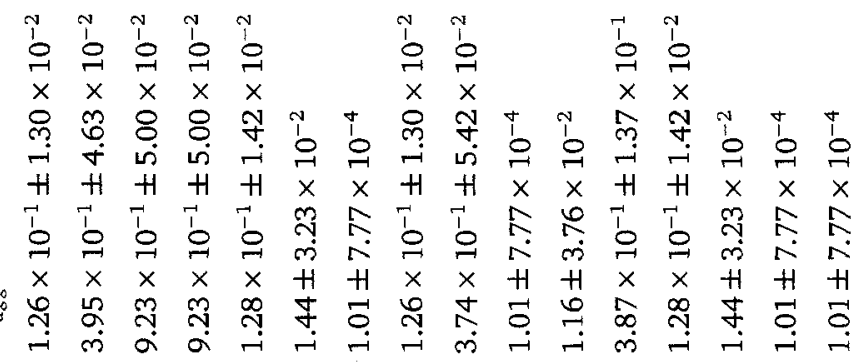

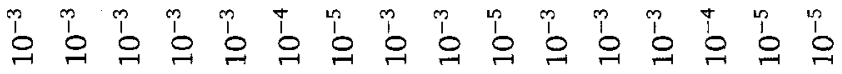

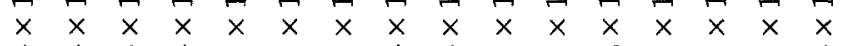

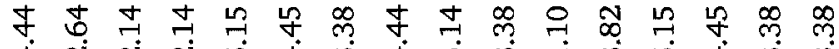

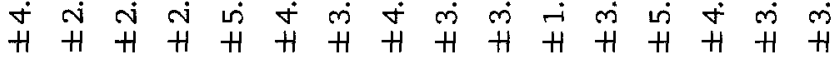

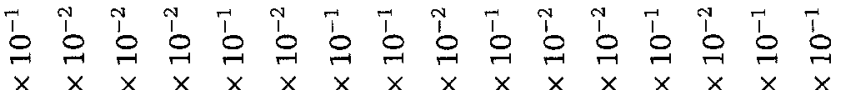
ঋ)

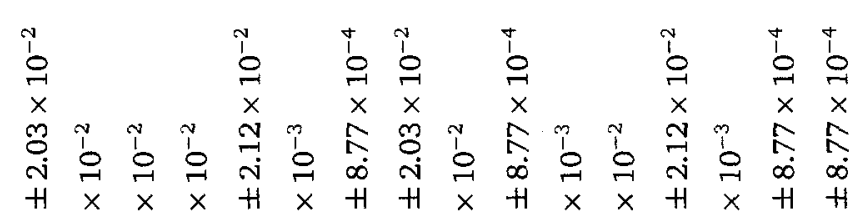

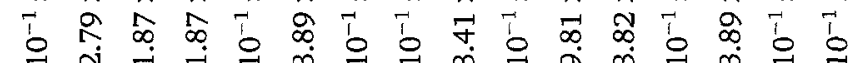

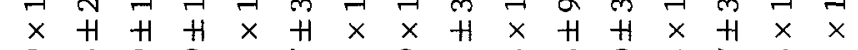

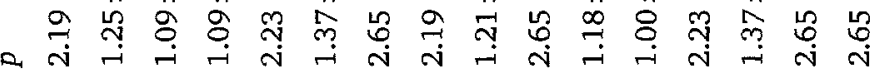

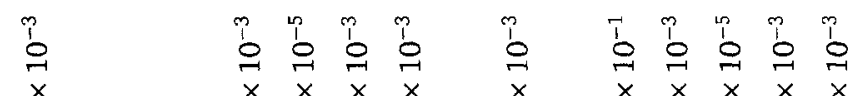

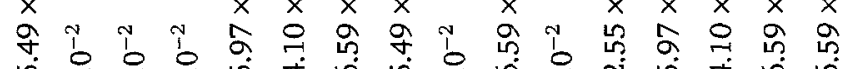

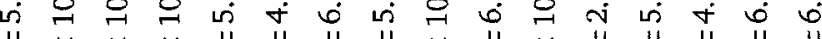

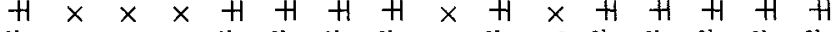
î

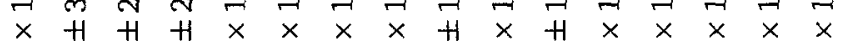

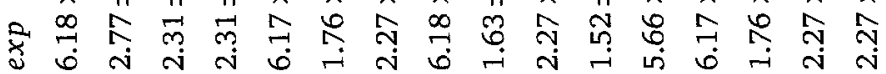

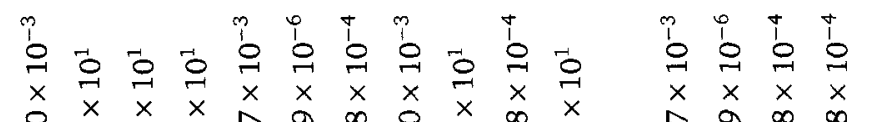

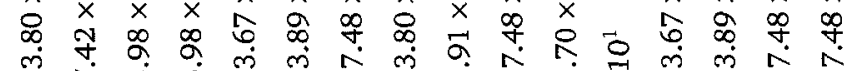

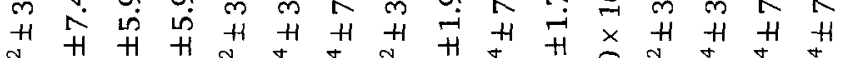

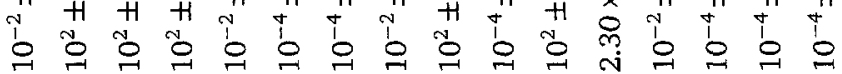

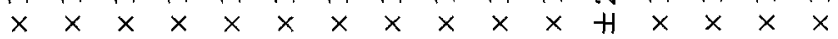

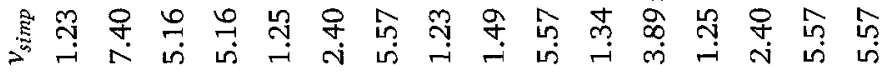

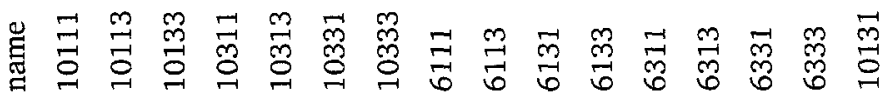




\begin{tabular}{|c|c|c|c|c|c|}
\hline name & $v_{\text {simp }}$ & $\exp$ & $p$ & $s_{a g g}$ & $t \theta_{a g g}$ \\
\hline 10111 & $3.08 \times 10^{-2} \pm 1.29 \times 10^{-2}$ & $8.86 \times 10^{-2} \pm 9.40 \times 10^{-3}$ & $7.21 \times 10^{-1} \pm 3.06 \times 10^{-1}$ & $1.39 \times 10^{-1} \pm 2.06 \times 10^{-3}$ & $8.96 \times 10^{-1} \pm 4.95 \times 10^{-1}$ \\
\hline 10113 & $8.82 \times 10^{2} \pm 7.37 \times 10^{1}$ & $2.94 \pm 4.72 \times 10^{-2}$ & $1.59 \pm 3.27 \times 10^{-2}$ & $6.48 \times 10^{-2} \pm 5.49 \times 10^{-3}$ & $2.50 \pm 3.36 \times 10^{-1}$ \\
\hline 10133 & $9.04 \times 10^{2} \pm 1.34 \times 10^{2}$ & $2.93 \pm 4.41 \times 10^{-2}$ & $1.59 \pm 2.66 \times 10^{-2}$ & $1.19 \times 10^{-1} \pm 5.76 \times 10^{-3}$ & $2.97 \pm 8.90 \times 10^{-2}$ \\
\hline 10311 & $3.16 \times 10^{-4} \pm 3.30 \times 10^{-5}$ & $1.81 \times 10^{-2} \pm 4.76 \times 10^{-4}$ & $1.52 \pm 4.53 \times 10^{-2}$ & $1.01 \times 10^{-1} \pm 3.82 \times 10^{-3}$ & $2.28 \pm 3.57 \times 10^{-1}$ \\
\hline 10313 & $3.20 \times 10^{-2} \pm 9.46 \times 10^{-3}$ & $8.86 \times 10^{-2} \pm 8.63 \times 10^{-3}$ & $7.67 \times 10^{-1} \pm 3.14 \times 10^{-1}$ & $1.39 \times 10^{-1} \pm 1.65 \times 10^{-3}$ & $1.02 \pm 5.97 \times 10^{-1}$ \\
\hline 10331 & $5.32 \times 10^{-4} \pm 4.73 \times 10^{-5}$ & $2.30 \times 10^{-2} \pm 6.88 \times 10^{-4}$ & $1.57 \pm 1.72 \times 10^{-2}$ & $6.36 \times 10^{-2} \pm 5.59 \times 10^{-3}$ & $3.05 \pm 4.19 \times 10^{-2}$ \\
\hline 10333 & $1.09 \times 10^{-3} \pm 1.03 \times 10^{-3}$ & $2.91 \times 10^{-2} \pm 6.86 \times 10^{-3}$ & $2.87 \times 10^{-1} \pm 3.16 \times 10^{-3}$ & $1.44 \times 10^{-1} \pm 1.08 \times 10^{-4}$ & $1.41 \pm 2.88 \times 10^{-1}$ \\
\hline 6111 & $3.08 \times 10^{-2} \pm 1.29 \times 10^{-2}$ & $8.86 \times 10^{-2} \pm 9.40 \times 10^{-3}$ & $7.21 \times 10^{-1} \pm 3.06 \times 10^{-1}$ & $1.39 \times 10^{-1} \pm 2.06 \times 10^{-3}$ & $8.96 \times 10^{-1} \pm 4.95 \times 10^{-1}$ \\
\hline 6113 & $1.75 \times 10^{2} \pm 1.80 \times 10^{1}$ & $1.72 \pm 2.44 \times 10^{-2}$ & $1.59 \pm 3.57 \times 10^{-2}$ & $6.56 \times 10^{-2} \pm 4.59 \times 10^{-3}$ & $2.43 \pm 4.01 \times 10^{-1}$ \\
\hline 6131 & $1.09 \times 10^{-3} \pm 1.03 \times 10^{-3}$ & $2.91 \times 10^{-2} \pm 6.86 \times 10^{-3}$ & $2.87 \times 10^{-1} \pm 3.16 \times 10^{-3}$ & $1.44 \times 10^{-1} \pm 1.08 \times 10^{-4}$ & $1.41 \pm 2.88 \times 10^{-1}$ \\
\hline 6133 & $1.80 \times 10^{2} \pm 2.66 \times 10^{1}$ & $1.70 \pm 2.23 \times 10^{-2}$ & $1.59 \pm 3.50 \times 10^{-2}$ & $1.17 \times 10^{-1} \pm 6.55 \times 10^{-3}$ & $3.00 \pm 6.68 \times 10^{-2}$ \\
\hline 6311 & $3.11 \times 10^{-4} \pm 3.24 \times 10^{-5}$ & $1.82 \times 10^{-2} \pm 4.42 \times 10^{-4}$ & $1.51 \pm 2.62 \times 10^{-2}$ & $1.01 \times 10^{-1} \pm 2.59 \times 10^{-3}$ & $2.36 \pm 4.03 \times 10^{-1}$ \\
\hline 6313 & $3.20 \times 10^{-2} \pm 9.46 \times 10^{-3}$ & $8.86 \times 10^{-2} \pm 8.63 \times 10^{-3}$ & $7.67 \times 10^{-1} \pm 3.14 \times 10^{-1}$ & $1.39 \times 10^{-1} \pm 1.65 \times 10^{-3}$ & $1.02 \pm 5.97 \times 10^{-1}$ \\
\hline 6331 & $5.32 \times 10^{-4} \pm 4.73 \times 10^{-5}$ & $2.30 \times 10^{-2} \pm 6.88 \times 10^{-4}$ & $1.57 \pm 1.72 \times 10^{-2}$ & $6.36 \times 10^{-2} \pm 5.59 \times 10^{-3}$ & $3.05 \pm 4.19 \times 10^{-2}$ \\
\hline 6333 & $1.09 \times 10^{-3} \pm 1.03 \times 10^{-3}$ & $2.91 \times 10^{-2} \pm 6.86 \times 10^{-3}$ & $2.87 \times 10^{-1} \pm 3.16 \times 10^{-3}$ & $1.44 \times 10^{-1} \pm 1.08 \times 10^{-4}$ & $1.41 \pm 2.88 \times 10^{-1}$ \\
\hline 10131 & $1.09 \times 10^{-3} \pm 1.03 \times 10^{-3}$ & $2.91 \times 10^{-2} \pm 6.86 \times 10^{-3}$ & $2.87 \times 10^{-1} \pm 3.16 \times 10^{-3}$ & $1.44 \times 10^{-1} \pm 1.08 \times 10^{-4}$ & $1.41 \pm 2.88 \times 10^{-1}$ \\
\hline
\end{tabular}

Table 31: Maximum values for simple Attraction Repulsion model 


\section{Appendix B}

\section{Linear Models For Prey and Predator Scenarios}

The tables in this appendix contain the standardised coefficients for the linear models calculated for each of the aggregate level measures. For each aggregate measure (each column), the standardised coefficient for each of the individual measures is provided, showing whether or not each of these measures contributes significantly and, if so, how much each measure contributes relative to other measures.

$\begin{array}{lllllllll} & v_{\text {simp }} & \exp & p & n_{\text {groups }} & n_{\text {strag }} & n_{\text {clust }} & s_{\text {agg }} & t \theta_{\text {agg }} \\ \text { space }_{\text {pref }} & 0.13 & 0.154 & 0.025 & \mathrm{~ns} & 0.076 & 0.057 & 0.014 & \mathrm{~ns} \\ w_{c} & -0.129 & -0.275 & 0.301 & -0.352 & -0.37 & -0.381 & 0.244 & 0.103 \\ w_{v} & -0.153 & -0.3 & -0.347 & -0.357 & -0.364 & -0.37 & 0.2 & 0.228 \\ w_{s} & 0.141 & 0.167 & 0.091 & 0.095 & 0.13 & 0.129 & -0.036 & 0.129 \\ s_{\max } & 0.116 & 0.142 & -0.39 & 0.201 & 0.102 & 0.12 & 0.815 & -0.682 \\ t \theta_{\max } & \mathrm{ns} & \mathrm{ns} & \mathrm{ns} & 0.015 & \mathrm{~ns} & \mathrm{~ns} & \mathrm{~ns} & \mathrm{~ns}\end{array}$

Table 32: Prey: Linear model of relationship between individual properties and aggregate properties 


$\begin{array}{lllllllll} & v_{\text {simp }} & \exp & p & n_{\text {groups }} & n_{\text {strag }} & n_{\text {clust }} & s_{\text {agg }} & t \theta_{\text {agg }} \\ \text { space }_{\text {pref }} & \mathrm{ns} & \mathrm{ns} & 0.061 & \mathrm{~ns} & 0.09 & 0.066 & \mathrm{~ns} & \mathrm{~ns} \\ w_{c} & 0.174 & 0.257 & 0.145 & -0.186 & -0.415 & -0.393 & 0.099 & 0.028 \\ w_{v} & -0.316 & -0.42 & -0.322 & -0.4 & -0.267 & -0.304 & 0.369 & 0.339 \\ w_{s} & \mathrm{~ns} & \mathrm{~ns} & 0.151 & 0.103 & 0.152 & 0.15 & -0.031 & 0.121 \\ s_{\max } & 0.335 & 0.45 & -0.483 & 0.288 & 0.069 & 0.12 & 0.747 & -0.665 \\ t \theta_{\max } & 0.043 & -0.015 & 0.016 & 0.026 & 0.05 & 0.055 & \mathrm{~ns} & 0.017\end{array}$

Table 33: Predator: Linear model of relationship between individual properties and aggregate properties 